Electronic Supporting Information

\title{
Base-Free and Bisphosphine Ligand Dialkylmanganese(II) Complexes as Precursors for Manganese Metal Deposition
}

Jeffrey S. Price, Preeti Chadha, David J. H. Emslie*

Department of Chemistry, McMaster University, 1280 Main Street West, Hamilton, Ontario, L8S 4M1, Canada. Fax: (905)-522-2509; Tel: (905)-525-9140 x 27699

E-mail: emslied@mcmaster.ca

Website: http://www.chemistry.mcmaster.ca/emslie/emslie.html 
ESI $1 \quad$ Evans Magnetic Measurement details $\quad$ S3-S4

$\begin{array}{lll}\text { ESI } 2 \text { SQUID calculations details } & \text { S5-S6 }\end{array}$

S1-S8 $\quad{ }^{1} \mathrm{H}$ NMR spectra of $\left[\mathrm{Mg}\left(\mathrm{CH}_{2} \mathrm{SiMe}_{3}\right)_{2}\right]$ and compounds $\mathbf{2}-\mathbf{8} \quad$ S7-S11

S9-S14 Variable temperature ${ }^{1}$ H NMR spectra of compounds $\mathbf{2}-\mathbf{5}$ and $\mathbf{7 - 8} \quad$ S12-S17

S15-S17 Variable temperature ${ }^{1} \mathrm{H}$ NMR spectra Evans measurements for 2, 5, $\quad$ S18-S20 and 7

S18-S26 2D Powder X-ray diffractograms of compounds $\mathbf{1}-\mathbf{8} \quad$ S21-S26

$\begin{array}{lll}\text { ESI } 3 & \text { SQUID data tables for complexes } \mathbf{2}-\mathbf{8} & \text { S27-S54 }\end{array}$

S27-S28 SQUID plots for complexes $3-\mathbf{4} \quad$ S55-S56

S29-S33 SQUID plots and fits to an exchange expression for $\mathbf{2}$ and $\mathbf{5}-\mathbf{8} \quad$ S57-S61

S34 UV/Vis spectra of compounds $\mathbf{2}-\mathbf{3}$ and $\mathbf{5}-\mathbf{8} \quad$ S62

S35-S37 X-ray crystal structures of $\left[\left\{\mathrm{Mn}\left(\mathrm{CH}_{2} \mathrm{SiMe}_{3}\right)\left(\mu-\mathrm{CH}_{2} \mathrm{SiMe}_{3}\right)\left(\mathrm{PEt}_{3}\right)\right\}_{2}\right], \quad$ S63-S64 5 (from hexanes), and $\mathbf{8}$

S38-S45 Crystallographic parameters of compounds $\mathbf{2}-\mathbf{7}$ and $\quad$ S65-S72 $\left[\left\{\mathrm{Mn}\left(\mathrm{CH}_{2} \mathrm{SiMe}_{3}\right)\left(\mu-\mathrm{CH}_{2} \mathrm{SiMe}_{3}\right)\left(\mathrm{PEt}_{3}\right)\right\}_{2}\right]$

S46-S55 $\quad{ }^{1} \mathrm{H}$ NMR spectra of results of $\mathrm{H}_{2}$ reactions with $\mathbf{1}-\mathbf{8} \quad$ S73-S77

S56-S63 $\quad{ }^{1} \mathrm{H}$ NMR spectra of results of $\mathrm{ZnEt}_{2}$ reactions with $\mathbf{1}-\mathbf{8} \quad$ S78-S82

S64-S67 PXRD diffractograms of $\mathrm{H}_{2}$ and $\mathrm{ZnEt}_{2}$ reactions with $\mathbf{1}-\mathbf{8} \quad$ S83-S84

S68-S71 XPS of powders deposited by $\mathrm{ZnEt}_{2}$ reactions with $\mathbf{2}$ and $7 \quad$ S85-S87

$\begin{array}{llr}\text { ESI } 4 & \text { References } & \text { S88 }\end{array}$ 


\section{ESI 1 Evans Magnetic Measurements for Solution Magnetic Susceptibility Details}

The equation used in the work was reported by Schubert. ${ }^{[1]}$ It is identical to that originally proposed by Evans, ${ }^{[2]}$ but modified for use with a superconducting magnet NMR spectrometer:

$$
\chi_{g}=\frac{-3(\Delta f)}{4 \pi f m}+\chi_{0}+\frac{\chi_{0}\left(d_{0}-d_{s}\right)}{m}
$$

$\chi_{g}=$ gram magnetic susceptibility $\left(\mathrm{cm}^{3} / \mathrm{g}\right)$

$\Delta \mathrm{f}=$ frequency shift of reference resonance $(\mathrm{Hz})$

$\mathrm{f}=$ spectrometer frequency $(\mathrm{Hz}$, not $\mathrm{MHz})$

$\mathrm{m}=$ grams of substance per $\mathrm{cm}^{3}$ of solution

$\chi_{0}=$ mass susceptibility of solvent $\left(\mathrm{cm}^{3} / \mathrm{g} ;-0.70 \times 10^{-6} \text { for benzene, }-0.71 \times 10^{-6} \text { for toluene }\right)^{[3]}$

$d_{0}=$ density of solvent $\left(\mathrm{g} / \mathrm{cm}^{3}\right)$

$d_{s}=$ density of solution $\left(\mathrm{g} / \mathrm{cm}^{3}\right)$

As noted by Evans, for highly paramagnetic substances, the last term of the equation can typically be ignored without introducing serious error. ${ }^{[2]}$

The equation used in this word was therefore:

$$
\chi_{g}=\frac{-3(\Delta f)}{4 \pi f m}+\chi_{0}
$$

Gram magnetic susceptibility was then converted to molar magnetic susceptibility $\left(\chi_{M}=\chi_{g} \times M\right)$, and a correction for the diamagnetic contribution to the magnetism was subsequently applied $\left(\chi_{M(\text { corr })}=\chi_{M}-\right.$ Diamag.Corr.). The diamagnetic contribution of a complex can be estimated by adding the tabulated diamagnetic contributions of the constituent atoms and $\pi$ bonds. ${ }^{[4]}$

When the complex is paramagnetic and obeys the Curie-Weiss law at the temperature measured, the effective magnetic moment can be calculated by:

$$
\mu_{e f f}=2.84 \sqrt{\frac{\chi_{M(c o r r)} \times(T-\theta)}{n}}
$$

$\chi_{M}=$ Molar magnetic susceptibility $\left(\mathrm{cm}^{3} / \mathrm{mol}\right)$

$\chi_{M(\text { corr })}=$ Molar magnetic susceptibility corrected for diamagnetic contributions to the magnetism $\left(\mathrm{cm}^{3} / \mathrm{mol}\right)$

$\mathrm{M}=$ molar mass $(\mathrm{g} / \mathrm{mol})$ 
$\mu_{e f f}=$ Effective magnetic moment per paramagnetic atom (B.M.)

$\mathrm{T}=$ Temperature $(\mathrm{K})$

$\theta=$ Weiss Constant $(\mathrm{K})$

$\mathrm{n}=$ number of paramagnetic atoms in the complex

The Weiss Constant is generally very small so can be ignored unless the temperature is extremely low (less than $100 \mathrm{~K}$ ). This leads to the simplified equation:

$$
\mu_{e f f}=2.84 \sqrt{\frac{\chi_{M(\text { corr })} \times(T)}{n}}
$$

From the effective magnetic moment, the number of unpaired electrons on the paramagnetic atom (N) can be predicted using the equation:

$$
\mu_{e f f}=\sqrt{N(N+2)}
$$

All Evans measurements were performed at $298 \mathrm{~K}$ without spinning, and the solvent was a 1:40 mixture of $\mathrm{C}_{6} \mathrm{H}_{6}: \mathrm{C}_{6} \mathrm{D}_{6}$ or a 1:40 mixture of toluene : $\mathrm{d}^{8}$ toluene. The sample solution was contained within a medium wall $4 \mathrm{~mm}$ O.D. Suprasil EPR tube with a Teflon valve (Wilmad LVP tube). This quartz tube was anchored (by a ring of Teflon tape) within a 5mm O.D. thin wall borosilicate NMR tube (4.2mm I.D.) containing pure solvent.

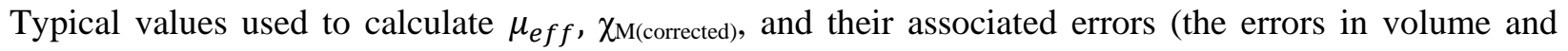
applied frequency are small enough to ignore relative to the much larger errors associated with compound mass, change in frequency, and temperature):

Compound mass $=9.6+/-0.5 \mathrm{mg}$

Volume $=1.87 \mathrm{~mL}$

Temperature $=298+/-1 \mathrm{~K}$

$\Delta f=-443.44+/-3.0 \mathrm{~Hz}$

$f=500000000 \mathrm{~Hz}(500 \mathrm{MHz})$

Using these values, the error associated with $\mu_{e f f}$ is $+/-0.16$ B.M and the error associated with $\chi_{\mathrm{M} \text { (corr) }}$ is $+/-0.0014 \mathrm{~cm}^{3} / \mathrm{mol}$. Because values reported are the averages of 2 experiments, the errors provided in the text are half of the square root of the sum of the squares of the two individually calculated errors. 


\section{ESI 2 SQUID for Solid State Magnetic Susceptibility details}

The molar magnetic susceptibility was calculated from the long moment measured by SQUID at a constant field over temperatures ranging from 5 to $300 \mathrm{~K}$;

$\chi_{M}=\left(\frac{\text { Long Moment }(\mathrm{emu})}{\text { Field }(\mathrm{Oe})}\right) / n(\mathrm{~mol})$

From this value, the corrected molar magnetic susceptibility can be calculated as described above for Evans measurements. When the complex is paramagnetic in the absence of any antiferromagnetic coupling/exchange, the effective magnetic moment in erg/G can be calculated from the slope of $\chi^{-1}$ vs. T where the slope is the inverse Curie constant $\left(\mathrm{C}^{-1}\right)$;

$$
\mu_{e f f}(\operatorname{erg} / G)=\sqrt{\frac{3 k_{B}}{n \times N_{A} \times C^{-1}}}
$$

$\mathrm{k}_{\mathrm{B}}=$ Boltzmann Constant $\left(1.381 \times 10^{-16} \mathrm{erg} / \mathrm{K}\right)$

$\mathrm{n}=$ number of paramagnetic metal ions in the complex

$\mathrm{N}_{\mathrm{A}}=$ Avogadro's number $\left(6.022 \times 10^{23} \mathrm{~mol}^{-1}\right)$

$\mathrm{C}^{-1}=$ Inverse Curie constant for given system; is the slope of the $\chi^{-1} \mathrm{vs} . \mathrm{T}$ function (mol/ergK)

The effective magnetic moment in erg/G can be converted to the more common units of Bohr magnetons;

$$
\mu_{e f f}(B . M .)=\mu_{e f f}(\operatorname{erg} / G) / 9.274 \times 10^{-21}
$$

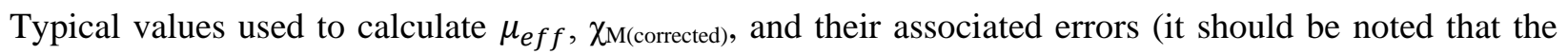
error associated with the long moment differs by about an order of magnitude for each scan even within the same sample):

Long Moment $=2.27 \times 10^{-4}+/-1.5 \times 10^{-8} \mathrm{emu}$

Compound mass $=57.2+/-0.5 \mathrm{mg}$

Temperature $=300 \mathrm{~K}$

Using these values, the error associated with $\chi_{\mathrm{M}(\mathrm{corr})}$ is $+/-0.00025 \mathrm{~cm}^{3} / \mathrm{mol}$. To determine the error in effective magnetic moment, both the standard deviation of the $\chi^{-1}$ vs. T slope and the error associated with mass must be taken into consideration:

$\mathrm{C}^{-1}=0.1175 \mathrm{~mol} / \mathrm{ergK}$

Std. dev. $\mathrm{C}^{-1}=0.00005 \mathrm{~mol} / \mathrm{ergK}$

Error due to mass $\mathrm{C}^{-1}=0.001 \mathrm{~mol} / \mathrm{ergK}$ 
Using these values (and those listed above regarding magnetic susceptibility), the error associated with $\mu_{\text {eff }}$ is $+/-0.03 \mathrm{BM}$

Complexes 2, and $\mathbf{5}-\mathbf{8}$, which feature antiferromagnetic exchange interactions between neighboring Mn atoms, were fit to a simple exchange expression using MAGMUN 4.1 ${ }^{[5]}$. Total spin values and their energies, based on the appropriate exchange Hamiltonian, are computed by MAGMUN4.1 ${ }^{[5]}$ and substituted into the van Vleck equation.

$$
\chi_{M^{\prime}}=\frac{N \beta^{2} g^{2}}{3 k(T-\theta)} \frac{\sum S^{\prime}\left(S^{\prime}+1\right)\left(2 S^{\prime}+1\right) e^{-E\left(S^{\prime}\right) / k T}}{\Sigma\left(2 S^{\prime}+1\right) e^{-E\left(S^{\prime}\right) / k T}}
$$

The fit required corrections to be applied for TIP (temperature independent paramagnetism), $\theta$ (Weisslike correction), and $\rho$ (paramagnetic impurity fraction).

$$
\chi_{M}=\chi_{M^{\prime}}(1-\rho)+\frac{4 S(S+1) N \beta^{2} g^{2} \rho}{3 k T}+T I P
$$


Figures S1-S7: $\quad$ NMR spectra of $\left[\mathrm{Mg}\left(\mathrm{CH}_{2} \underline{\mathrm{SiMe}}_{3} \underline{2}_{2}\right]\right.$ and compounds $2-8$

* For figures S2-S7, diamagnetic signals below 3 ppm are due to H-grease, Si-grease, or hexanes. These peaks appear relatively large despite making up a very low portion of the solution because they are diamagnetic and the complex signals are very broad due to being paramagnetic. The sharp signal at 7.16 ppm is due to residual $\mathrm{C}_{6} D_{5} \mathrm{H}$ solvent.

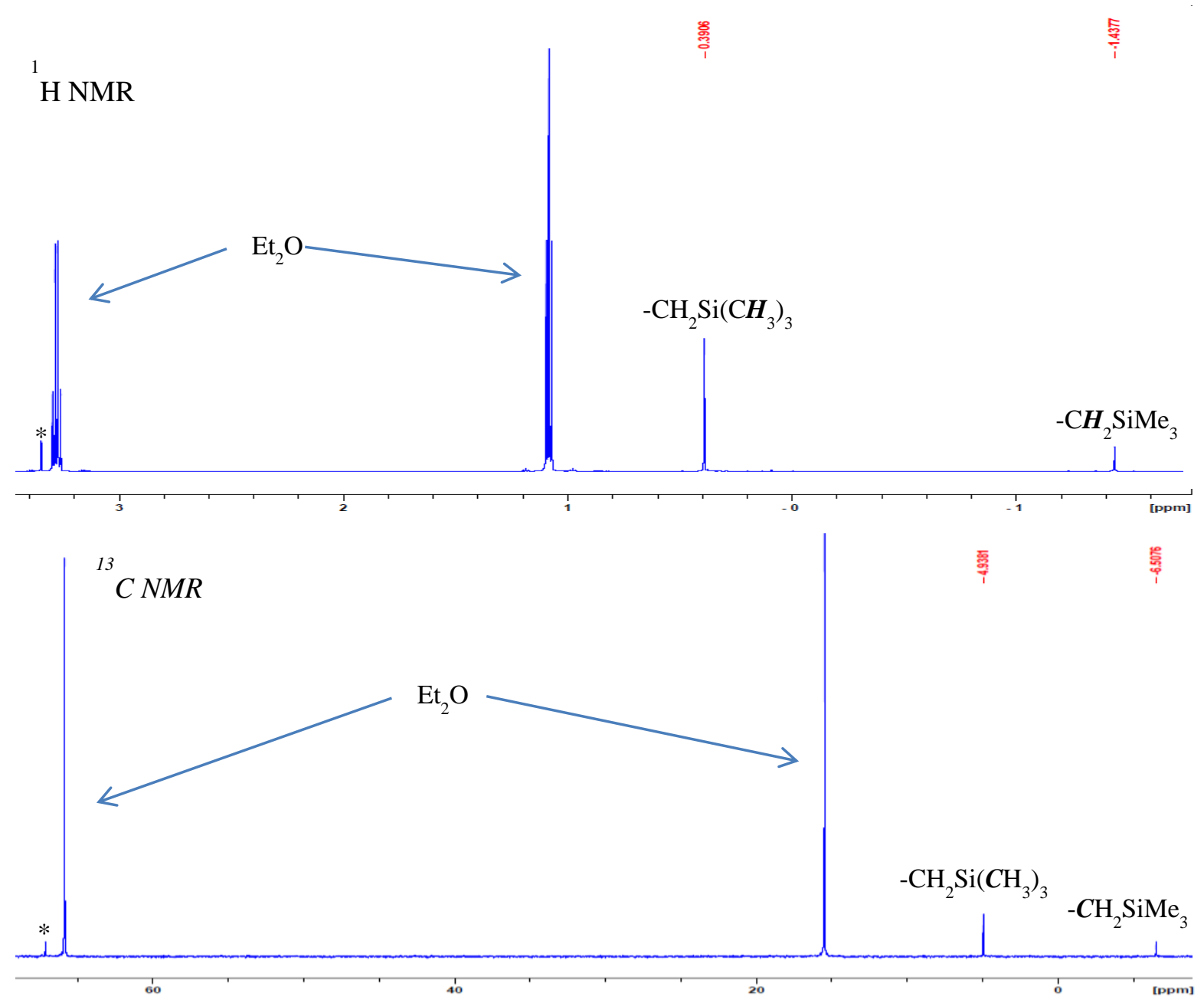

Figure S1: $\quad{ }^{1} \mathrm{H}(\mathrm{AV600})$ and ${ }^{13} \mathrm{C}$ NMR (AV500) spectra of $\left[\mathrm{Mg}\left(\mathrm{CH}_{2} \mathrm{SiMe}_{3}\right)_{2}\right]$ in $\mathrm{C}_{6} \mathrm{D}_{6}$ spiked with diethyl ether to improve solubility $(*=1,4$ - dioxane) 


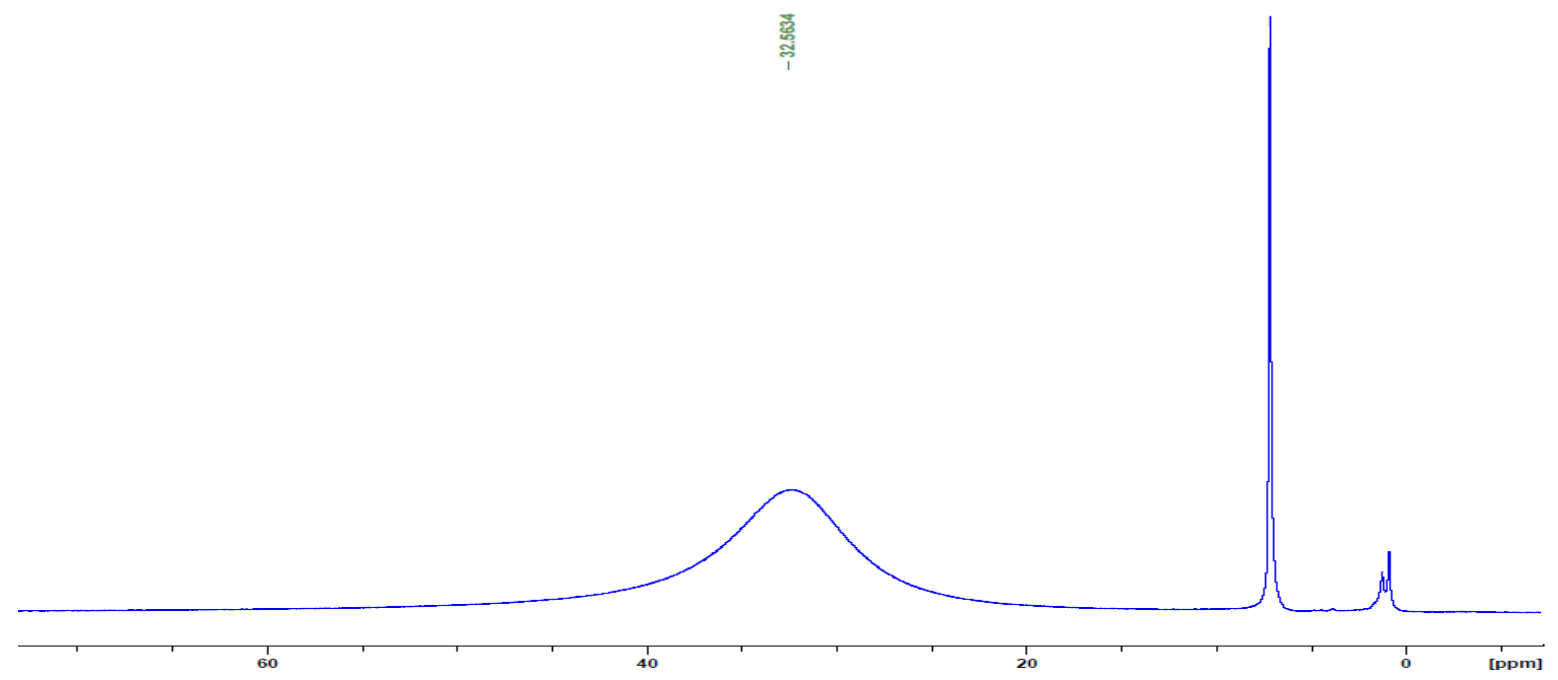

Figure S2: $\quad{ }^{1} \mathrm{H}$ NMR spectrum of $\left[\left\{\mathrm{Mn}\left(\mathrm{CH}_{2} \mathrm{CMe}_{3}\right)\left(\mu-\mathrm{CH}_{2} \mathrm{CMe}_{3}\right)_{2}\right\}_{2}\left\{\mathrm{Mn}\left(\mu-\mathrm{CH}_{2} \mathrm{CMe}_{3}\right)_{2} \mathrm{Mn}\right\}\right]$ (2) in $\mathrm{C}_{6} \mathrm{D}_{6}(500 \mathrm{MHz})$

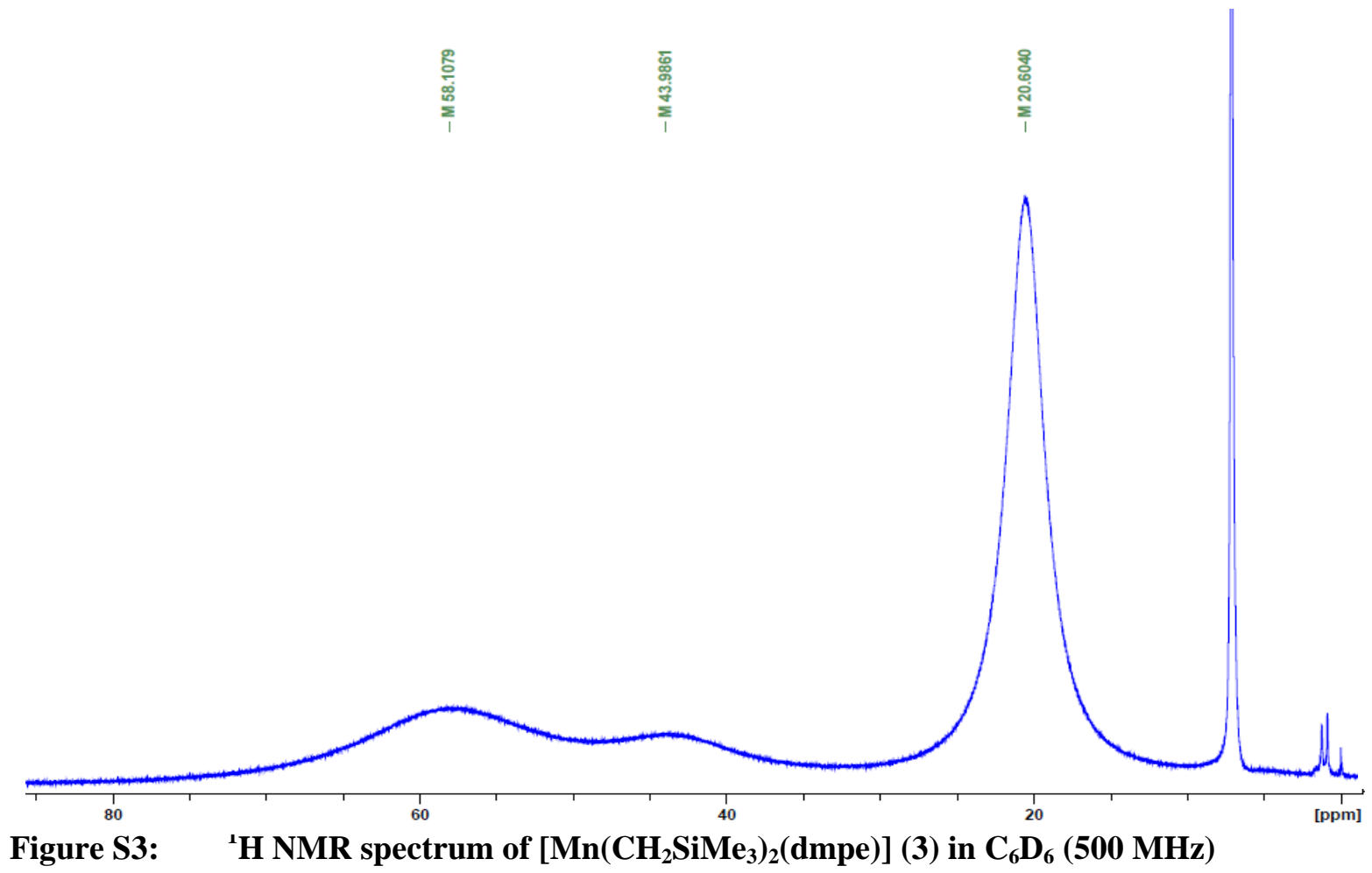




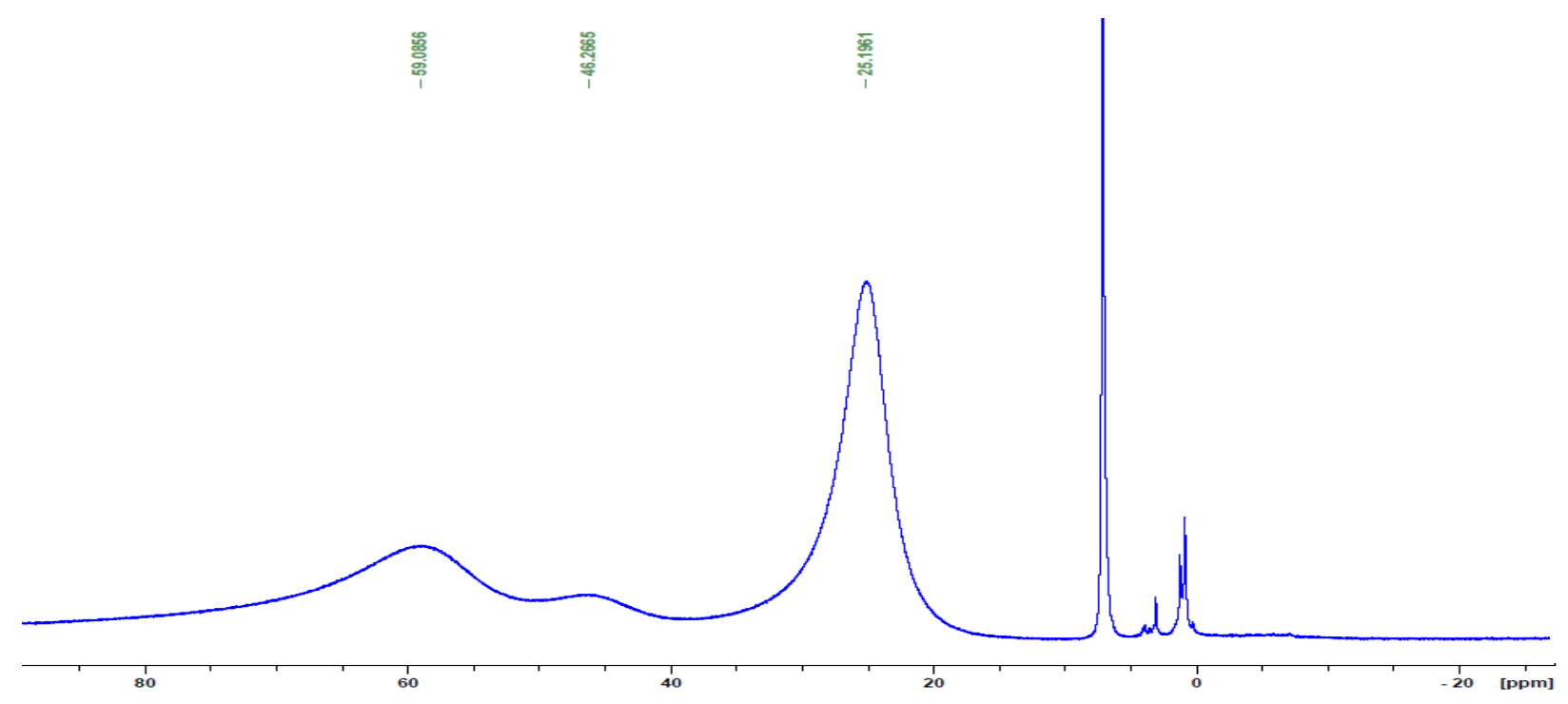

Figure S4: $\quad{ }^{1} \mathrm{H}$ NMR spectrum of $\left[\left\{\operatorname{Mn}\left(\mathrm{CH}_{2} \mathrm{CMe}_{3}\right)_{2}(\mu \text {-dmpe }\}_{2}\right](4)\right.$ in $\mathrm{C}_{6} \mathrm{D}_{6}(500 \mathrm{MHz})$

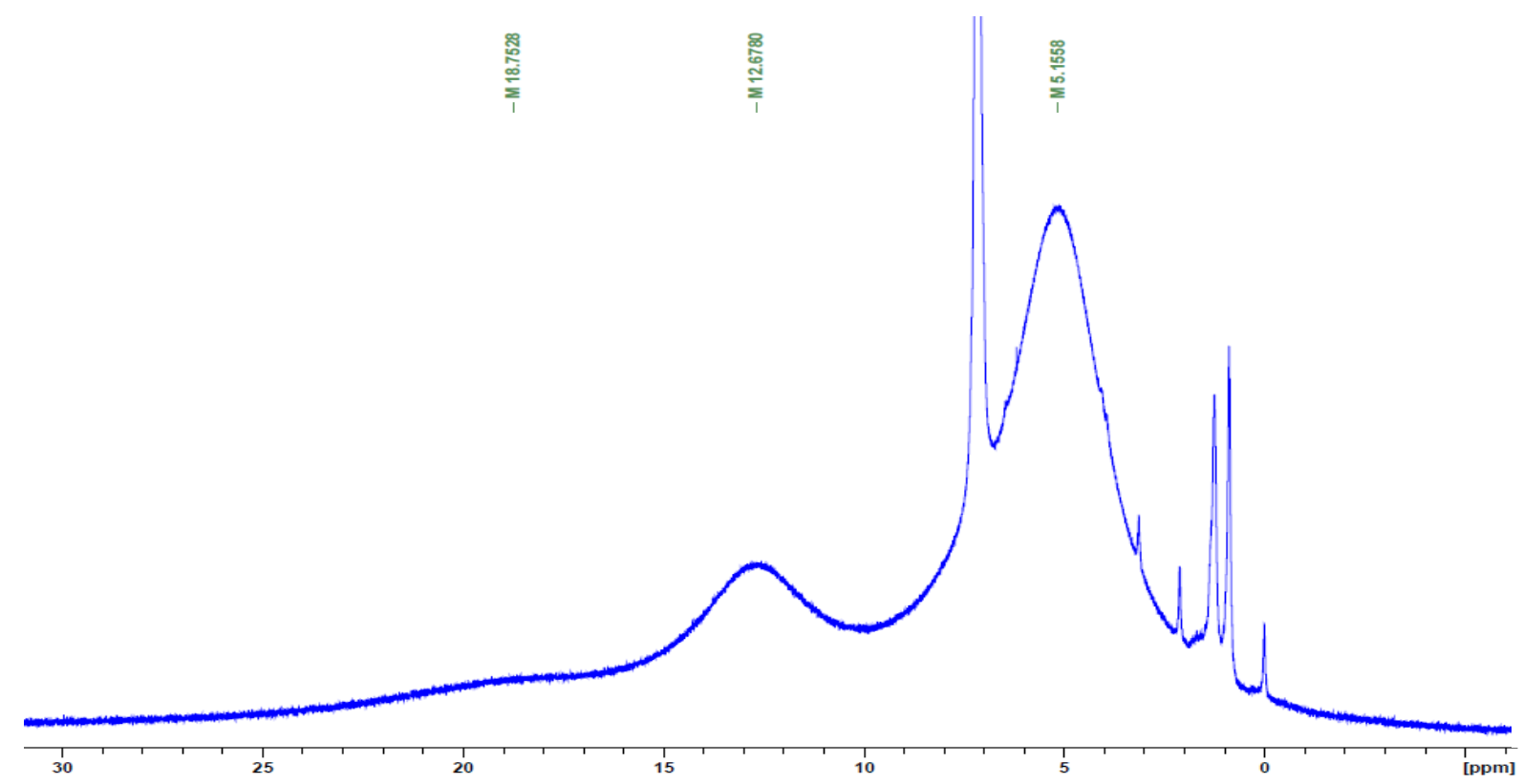

Figure S5: $\quad{ }^{1} \mathrm{H}$ NMR spectrum of $\left[\left\{\mathrm{Mn}\left(\mathrm{CH}_{2} \mathrm{SiMe}_{3}\right)\left(\mu-\mathrm{CH}_{2} \mathrm{SiMe}_{3}\right)\right\}_{2}(\mu-d m p e)\right](5)$ in $\mathrm{C}_{6} \mathrm{D}_{6}(500$ MHz) 


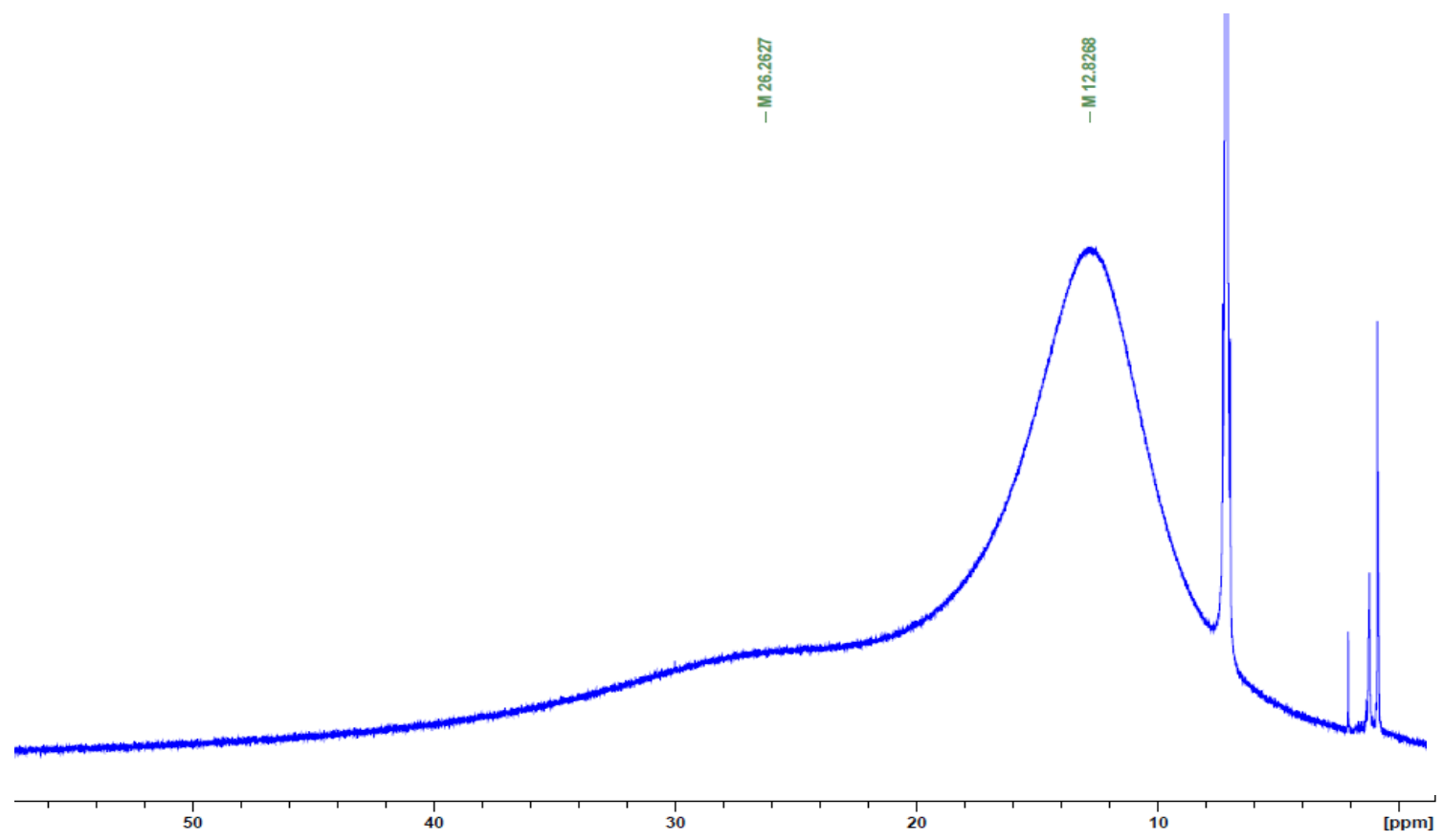

Figure S6: $\quad{ }^{1} \mathrm{H}$ NMR spectrum of $\left[\left\{\mathrm{Mn}\left(\mathrm{CH}_{2} \mathrm{CMe}_{3}\right)\left(\mu-\mathrm{CH}_{2} \mathrm{CMe}_{3}\right)\right\}_{2}(\mu\right.$-dmpe $\left.)\right](6)$ in $\mathrm{C}_{6} \mathrm{D}_{6}(500$ MHz)

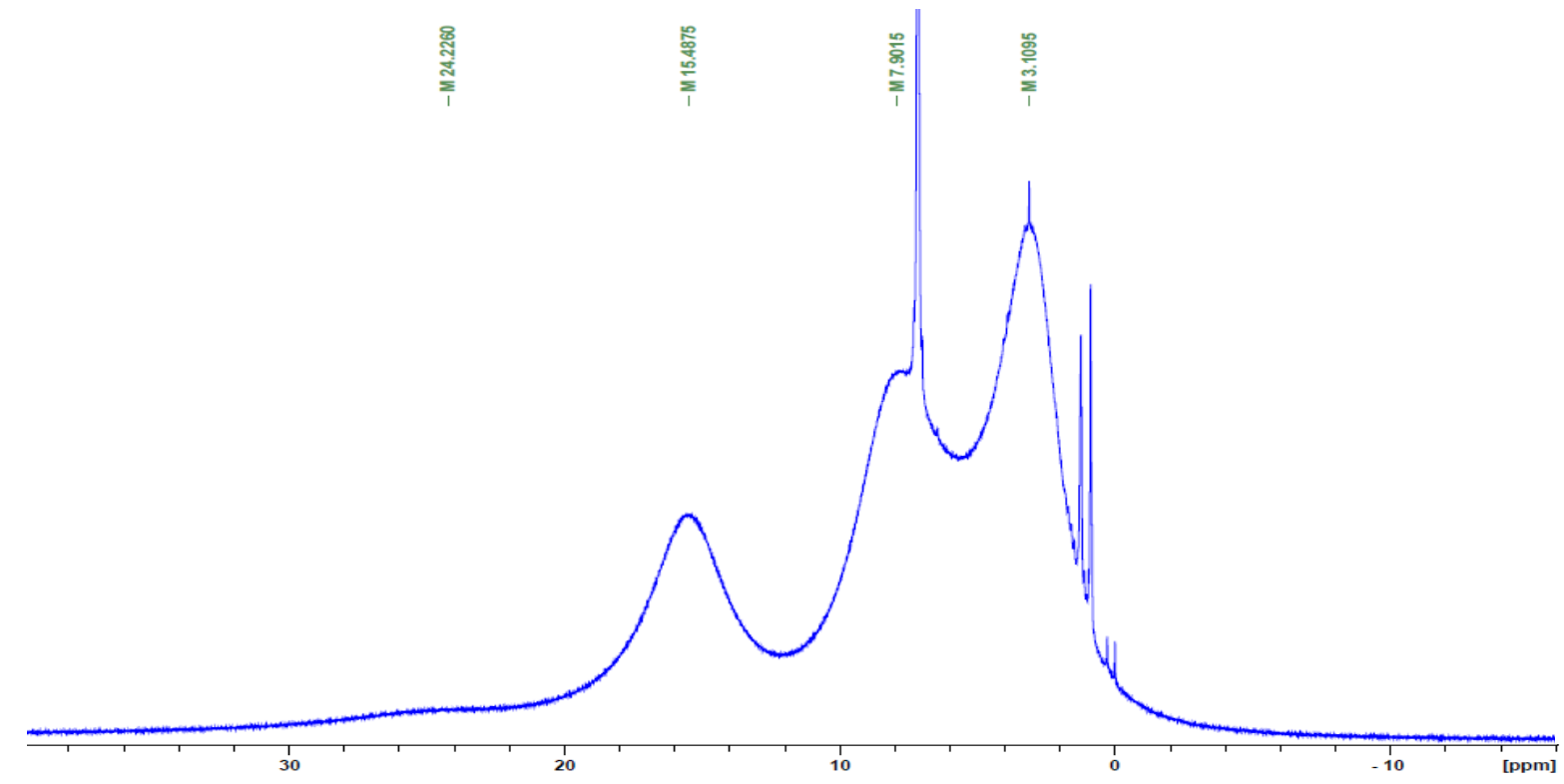

Figure S7: $\quad{ }^{1} \mathrm{H}$ NMR spectrum of $\left[\left\{\mathrm{Mn}\left(\mathrm{CH}_{2} \mathrm{SiMe}_{3}\right)\left(\mu-\mathrm{CH}_{2} \mathrm{SiMe}_{3}\right)\right\}_{2}(\mu-\mathrm{dmpm})\right](7)$ in $\mathrm{C}_{6} \mathrm{D}_{6}(500$ MHz) 


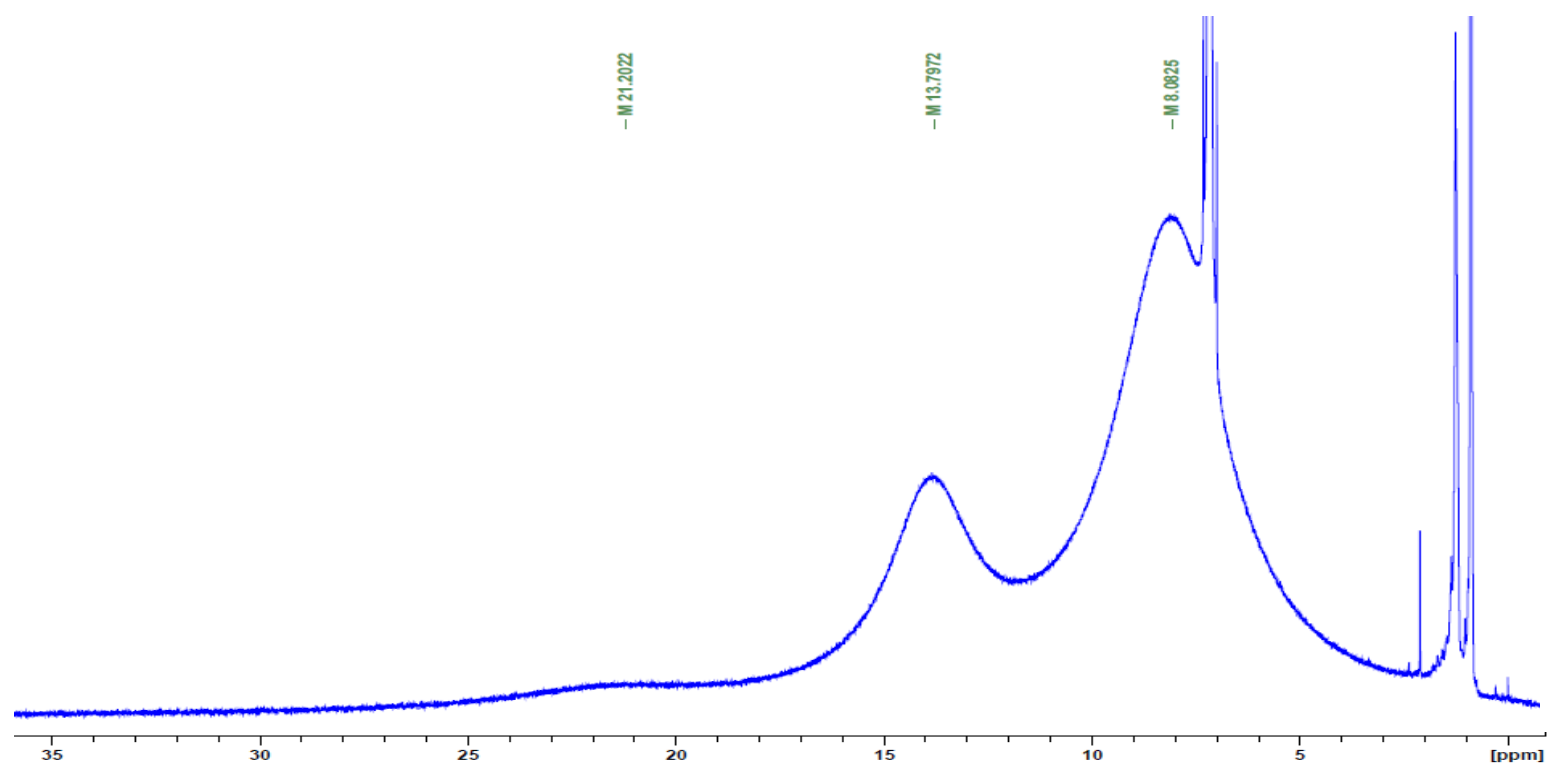

Figure S8: $\quad{ }^{1} \mathrm{H}$ NMR spectrum of $\left[\left\{\mathrm{Mn}\left(\mathrm{CH}_{2} \mathrm{CMe}_{3}\right)\left(\mu-\mathrm{CH}_{2} \mathrm{CMe}_{3}\right)\right\}_{2}(\mu-\mathrm{dmpm})\right](8)$ in $\mathrm{C}_{6} \mathrm{D}_{6}(500$ MHz) 
Figures S9-S14: $\quad$ Variable temperature ${ }^{1} \mathrm{H}$ NMR spectra of compounds $2-5$ and $7-8$

* For figures S11-S14, diamagnetic signals below 3 ppm are due to H-grease, Si-grease, or hexanes. These peaks appear relatively large despite making up a very low portion of the solution because they are diamagnetic and the complex signals are very broad due to being paramagnetic. The sharp signals at 2.08, 6.97, 7.01, and 7.09 ppm are due to residual $d^{7}$-toluene solvent.

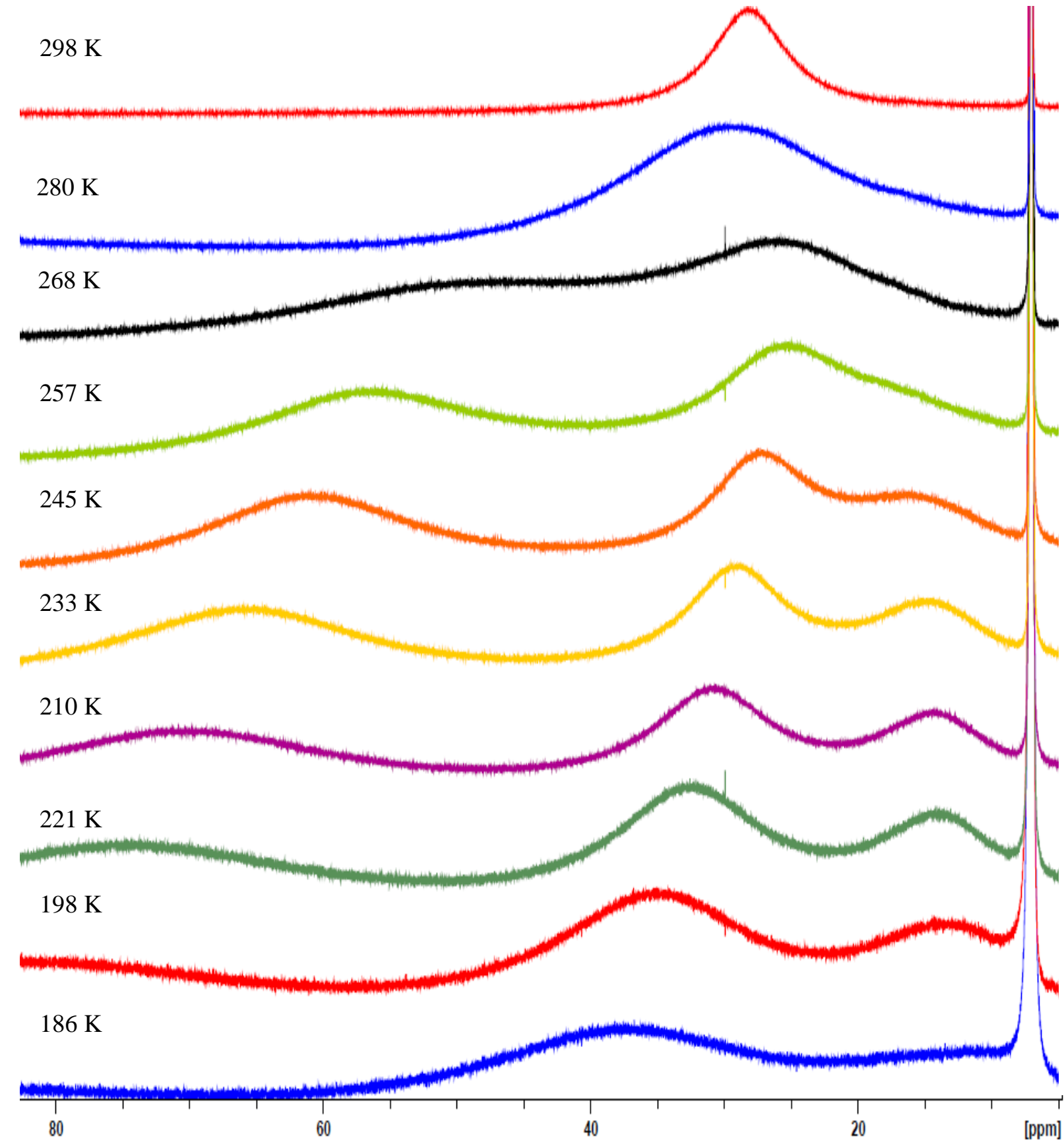

Figure S9: Variable temperature ${ }^{1} \mathrm{H} \quad \mathrm{NMR}$ of $\left[\left\{\mathrm{Mn}\left(\mathrm{CH}_{2} \mathrm{CMe}_{3}\right)\left(\mu-\mathrm{CH}_{2} \mathrm{CMe}_{3}\right)_{2}\right\}_{2}\{\mathrm{Mn}(\mu-\right.$ $\left.\left.\left.\mathrm{CH}_{2} \mathrm{CMe}_{3}\right)_{2} \mathrm{Mn}\right\}\right]$ (2) in $\mathrm{d}^{8}$-toluene (500 $\left.\mathrm{MHz}\right)$ 


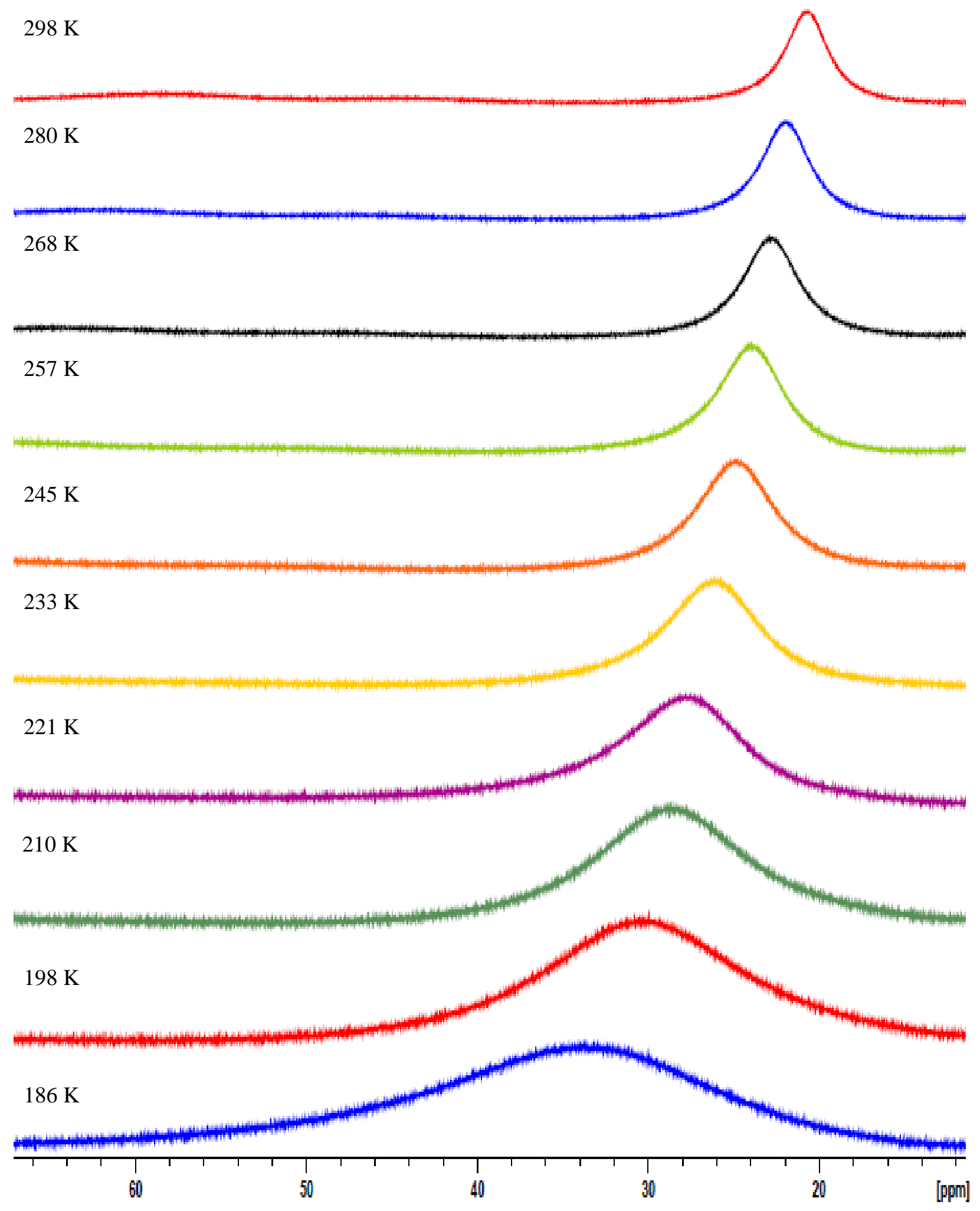

Figure S10: $\quad$ Variable temperature ${ }^{1} \mathrm{H}$ NMR of $\left[\mathrm{Mn}\left(\mathrm{CH}_{2} \mathrm{SiMe}_{3}\right)_{2}(\mathrm{dmpe})\right](3)$ in $\mathrm{d}^{8}$-toluene (500 MHz) 


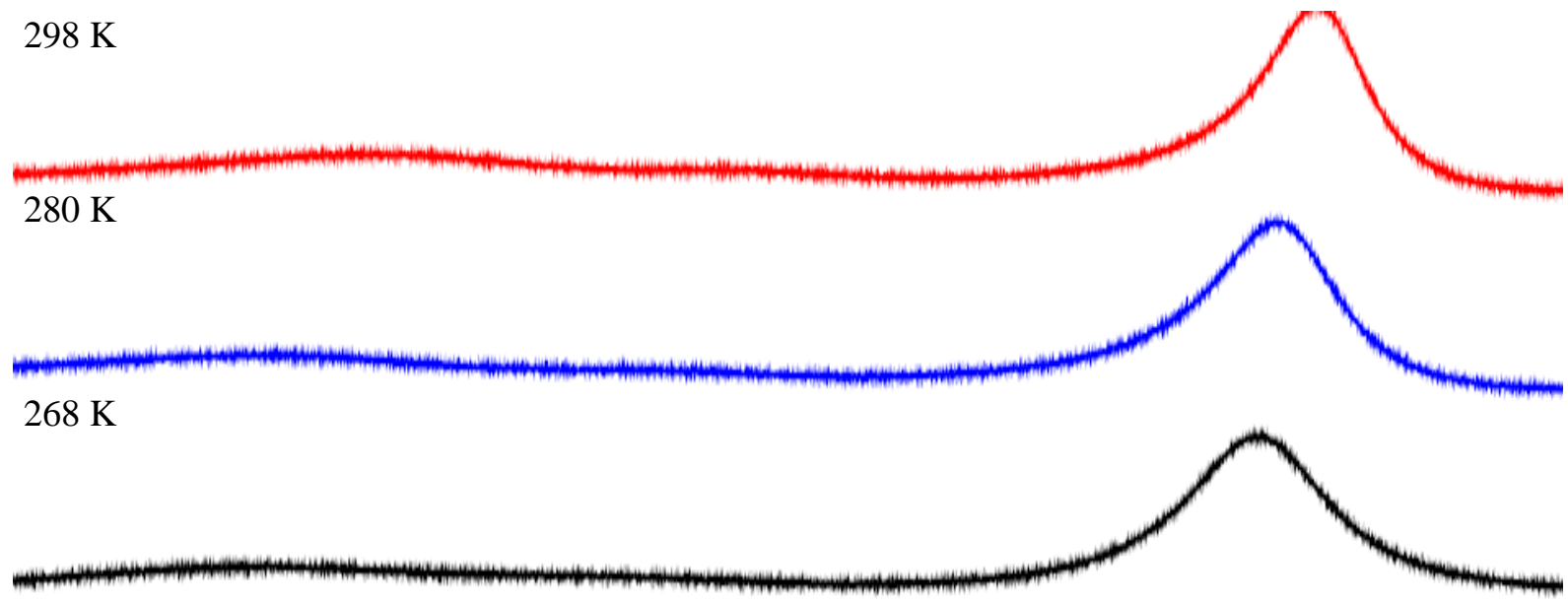
$257 \mathrm{~K}$

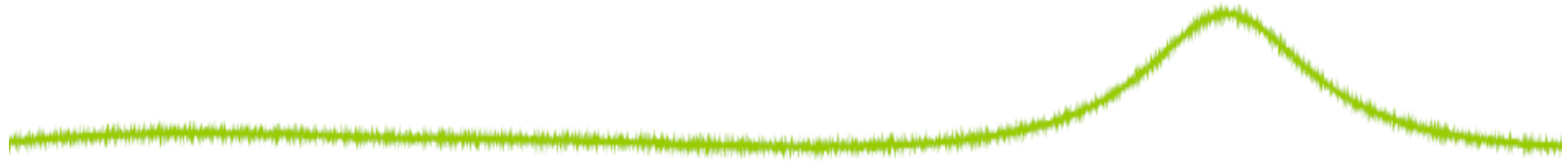

$245 \mathrm{~K}$

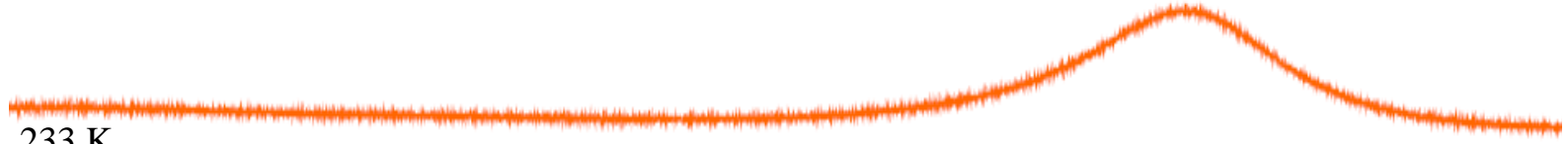
$233 \mathrm{~K}$
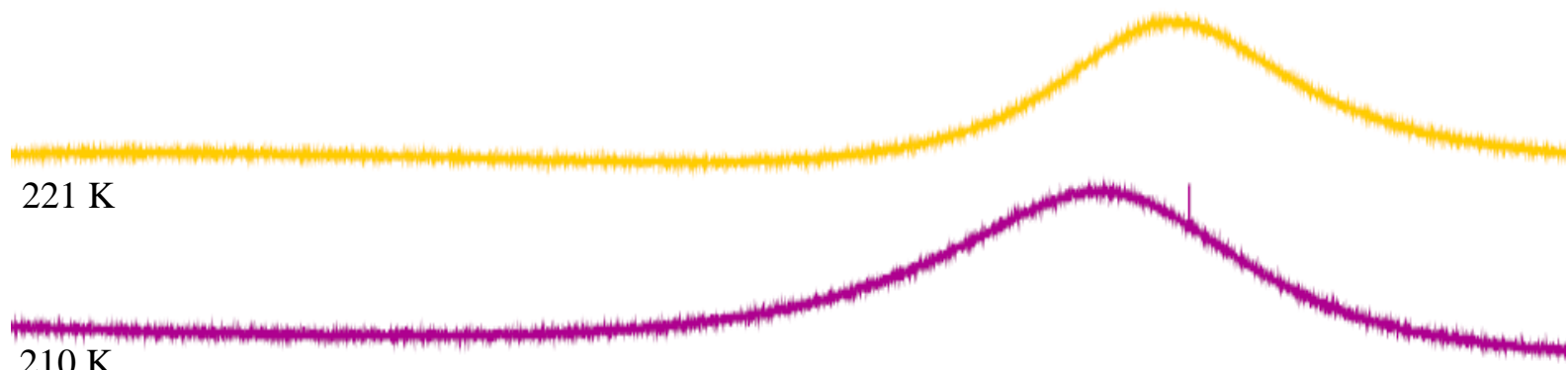
$210 \mathrm{~K}$

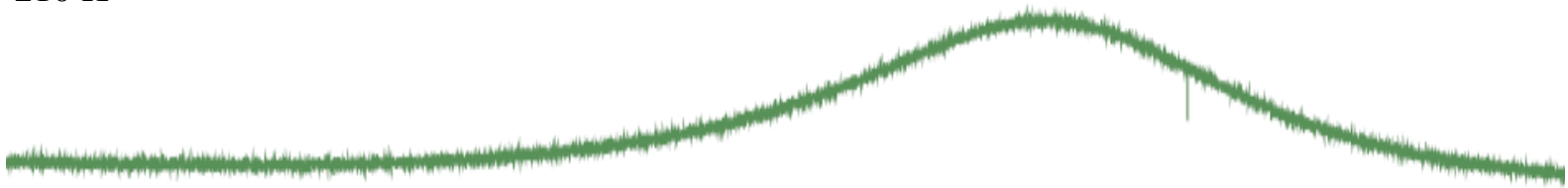
$198 \mathrm{~K}$

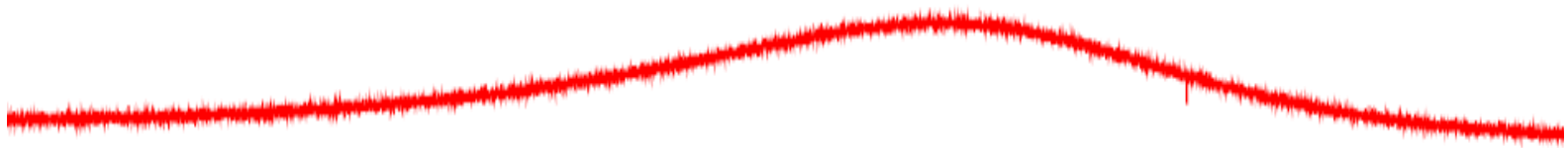
$186 \mathrm{~K}$

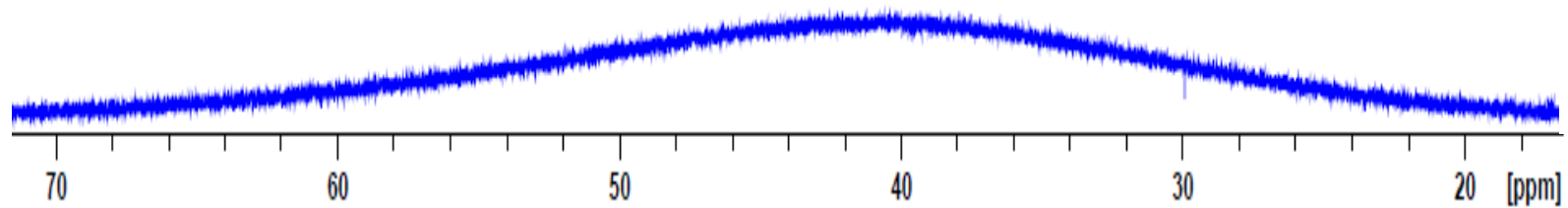

Figure S11: Variable temperature ${ }^{1} \mathrm{H}$ NMR of $\left[\left\{\mathrm{Mn}\left(\mathrm{CH}_{2} \mathrm{CMe}_{3}\right)_{2}(\mu-d m p e)\right\}_{2}\right](4)$ in $\mathrm{d}^{8}$-toluene (500 MHz) 

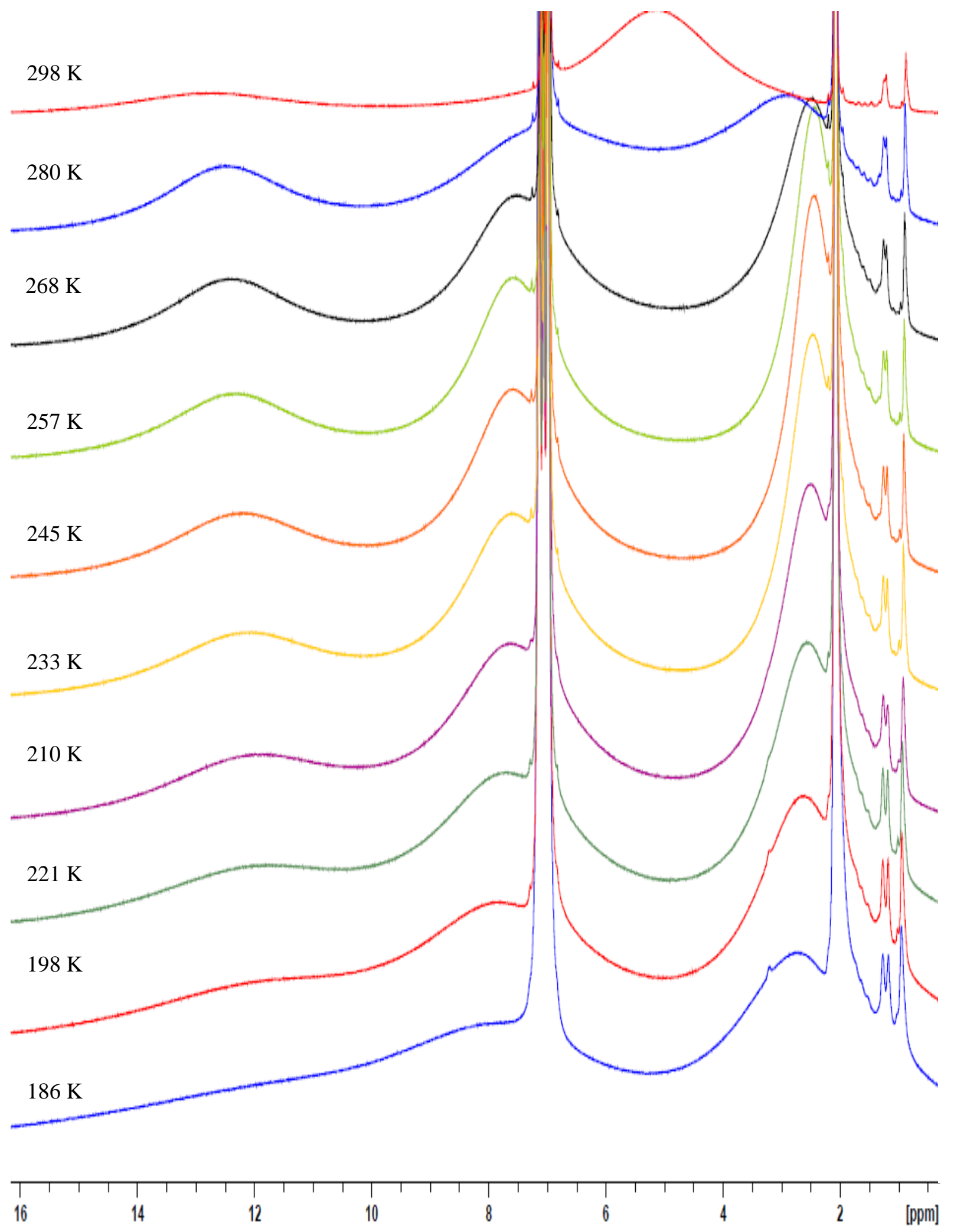

Figure S12: Variable temperature ${ }^{1} \mathrm{H}$ NMR of $\left[\left\{\mathrm{Mn}\left(\mathrm{CH}_{2} \mathrm{SiMe}_{3}\right)\left(\mu-\mathrm{CH}_{2} \mathrm{SiMe}_{3}\right)\right\}_{2}(\mu\right.$-dmpe) $(5)$ in $\mathrm{d}^{8}$-toluene (500 MHz) 


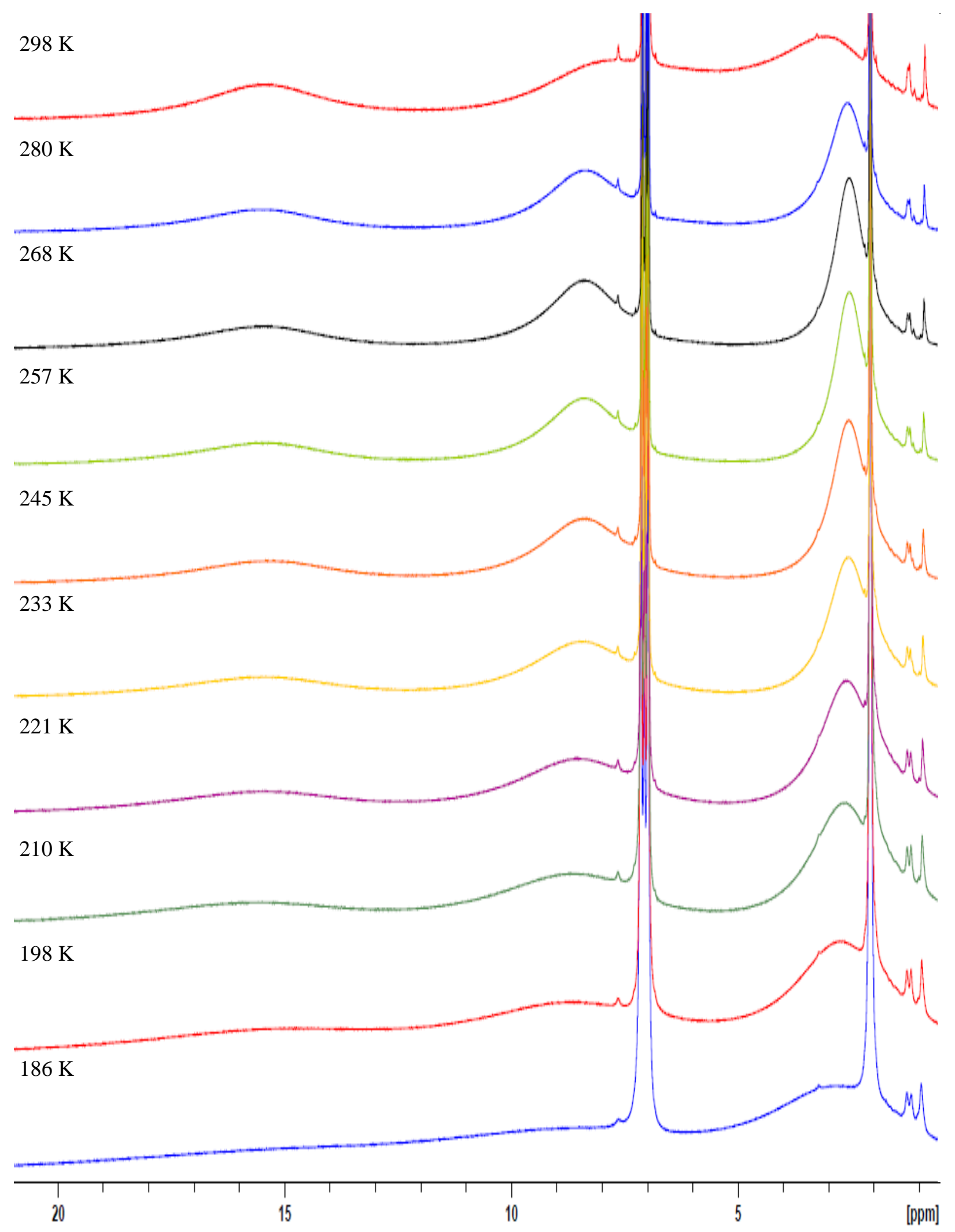

Figure S13: $\quad$ Variable temperature ${ }^{1} \mathrm{H}$ NMR of $\left[\left\{\mathrm{Mn}\left(\mathrm{CH}_{2} \mathrm{SiMe}_{3}\right)\left(\mu-\mathrm{CH}_{2} \mathrm{SiMe}_{3}\right)\right\}_{2}(\mu-\mathrm{dmpm})\right](7)$ in $\mathrm{d}^{8}$-toluene (500 MHz) 


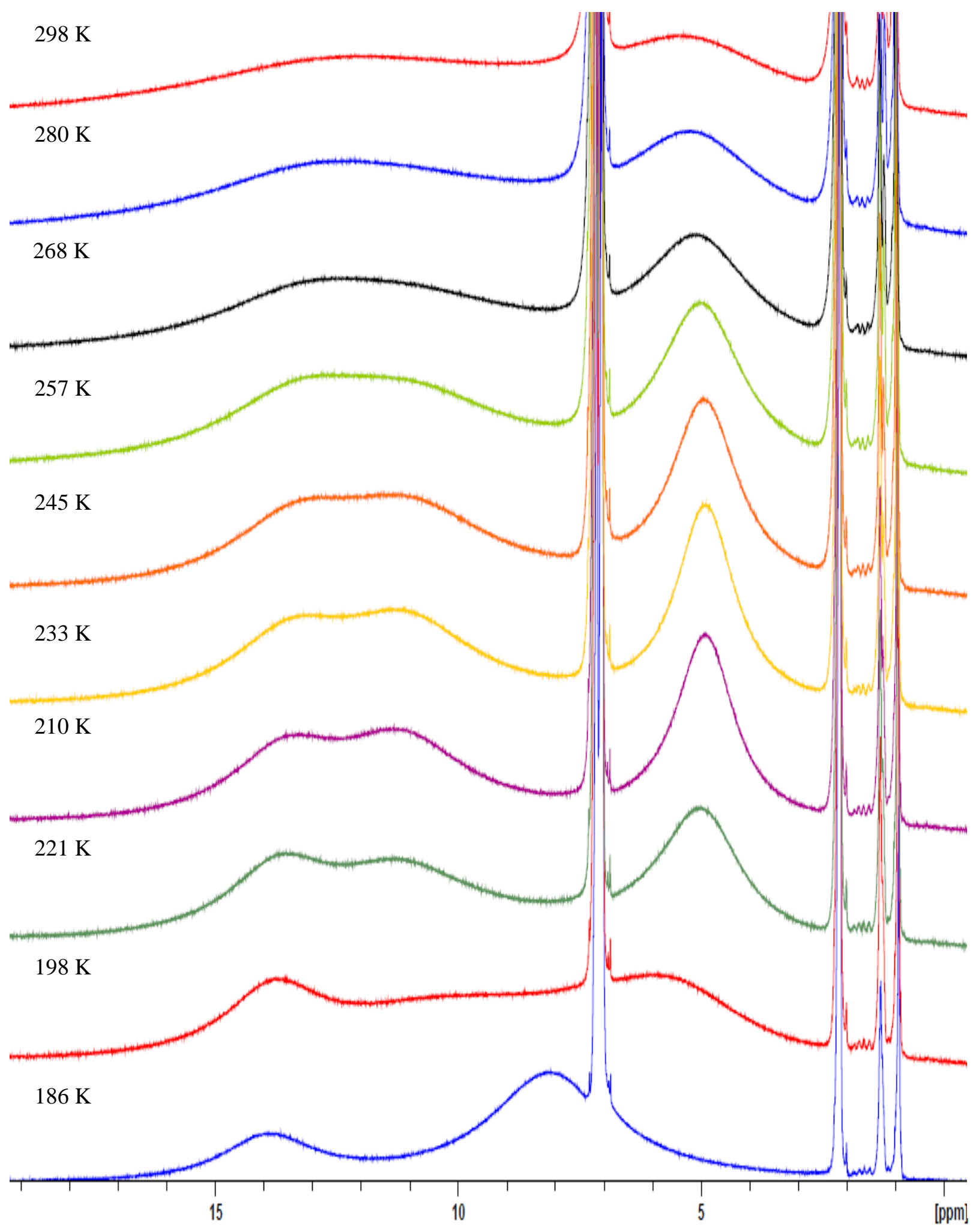

Figure S14: $\quad$ Variable temperature ${ }^{1} \mathrm{H}$ NMR of $\left[\left\{\mathrm{Mn}\left(\mathrm{CH}_{2} \mathrm{CMe}_{3}\right)\left(\mu-\mathrm{CH}_{2} \mathrm{CMe}_{3}\right)\right\}_{2}(\mu-\mathrm{dmpm})\right](8)$ in $\mathrm{d}^{8}$-toluene (500 MHz) 
Figures S15-S17: $\quad$ Variable temperature ${ }^{1}$ H NMR spectra Evans measurements for 2, 5, and 7

The signal at 2.11ppm is protonated toluene from the 40:1 mixture of $d^{8}$-toluene : toluene in the outer NMR tube (which does not contain any solute). The signal on the far left is protonated toluene from the $40: 1$ mixture of $d^{8}$-toluene : toluene in the inner NMR tube (which contains a dissolved organomanganese(II) complex). The difference between these two signals (in $\mathrm{Hz}$ ) is used for the pertinent calculations). Each signal has another of much lower intensity 0.03ppm to its right corresponding to residual $d^{7}$-toluene contained in the $d^{8}$-toluene of the respective $40: 1$ mixture.

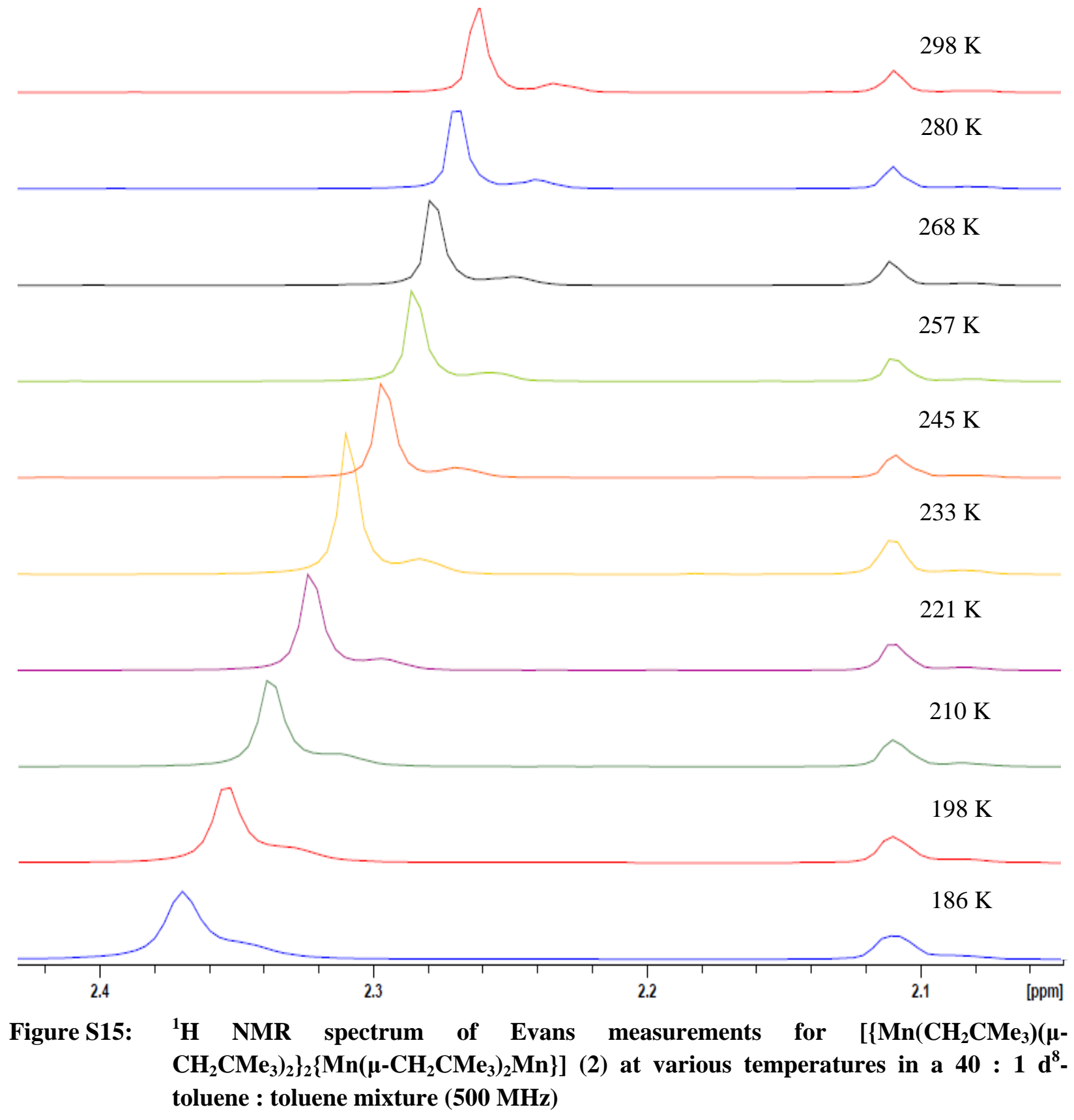



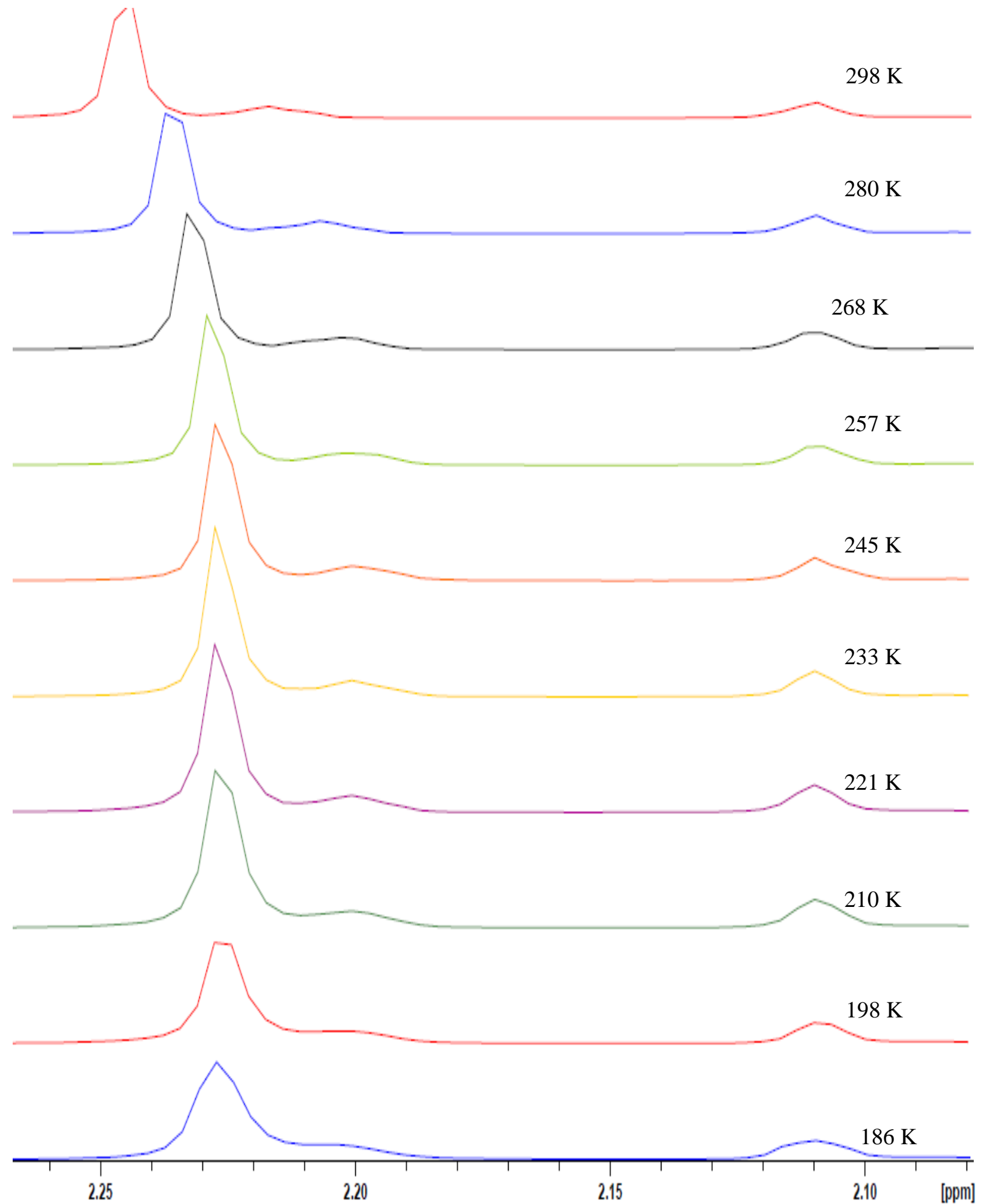

Figure S16: $\quad{ }^{1} \mathrm{H}$ NMR spectrum of Evans measurements for $\left[\left\{\mathrm{Mn}\left(\mathrm{CH}_{2} \mathrm{SiMe}_{3}\right)\left(\mu-\mathrm{CH}_{2} \mathrm{SiMe}_{3}\right)\right\}_{2}(\mu-\right.$ dmpe)] (5) at various temperatures in a $40: 1 \mathrm{~d}^{8}$-toluene : toluene mixture (500 MHz) 


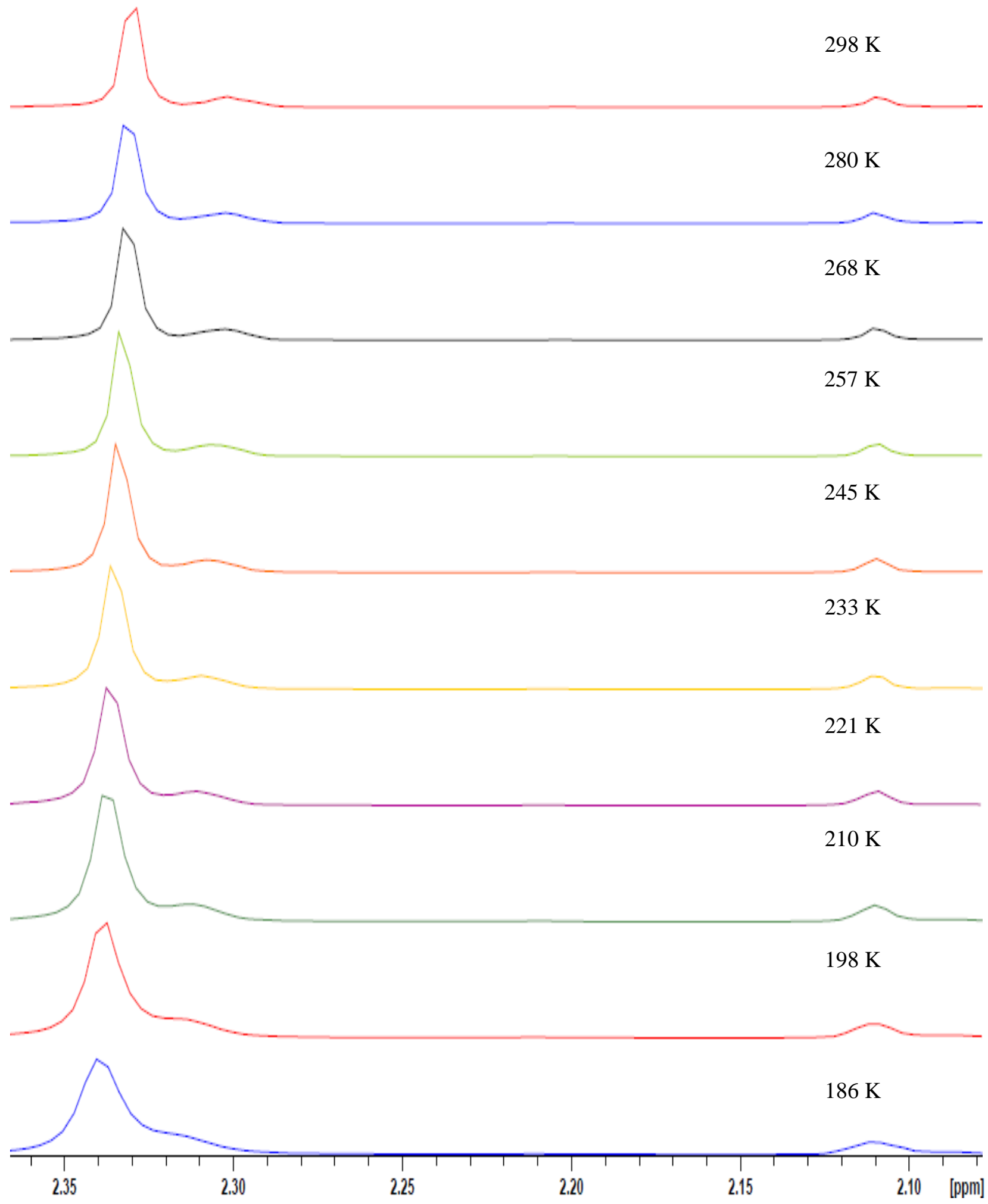

Figure S17: $\quad{ }^{1} \mathrm{H}$ NMR spectrum of Evans measurements for $\left[\left\{\mathrm{Mn}\left(\mathrm{CH}_{2} \mathrm{SiMe}_{3}\right)\left(\mu-\mathrm{CH}_{2} \mathrm{SiMe}_{3}\right)\right\}_{2}(\mu-\right.$ dmpm)] (7) at various temperatures in a $40: 1 \mathrm{~d}^{8}$-toluene : toluene mixture (500 MHz) 
Figures S18-S26: $\quad$ 2D Powder X-ray diffractograms of compounds 1 - 8

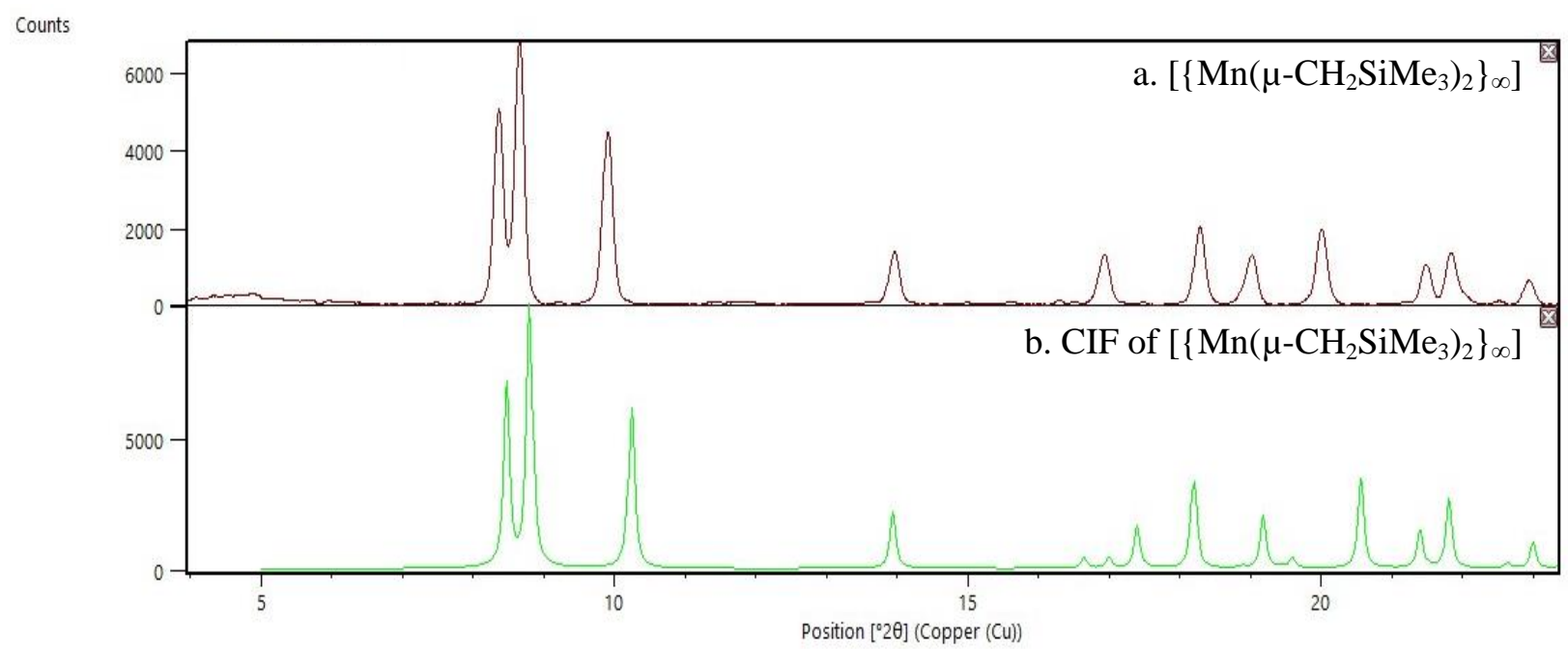

Figure S18: 2D Powder X-ray diffractograms of a. compound 1 (300 K), and b. compound 1 generated from .CIF (from literature) ${ }^{[6]}(150 \mathrm{~K})(\mathrm{Cu}$ source $\lambda=1.54056 \AA)$

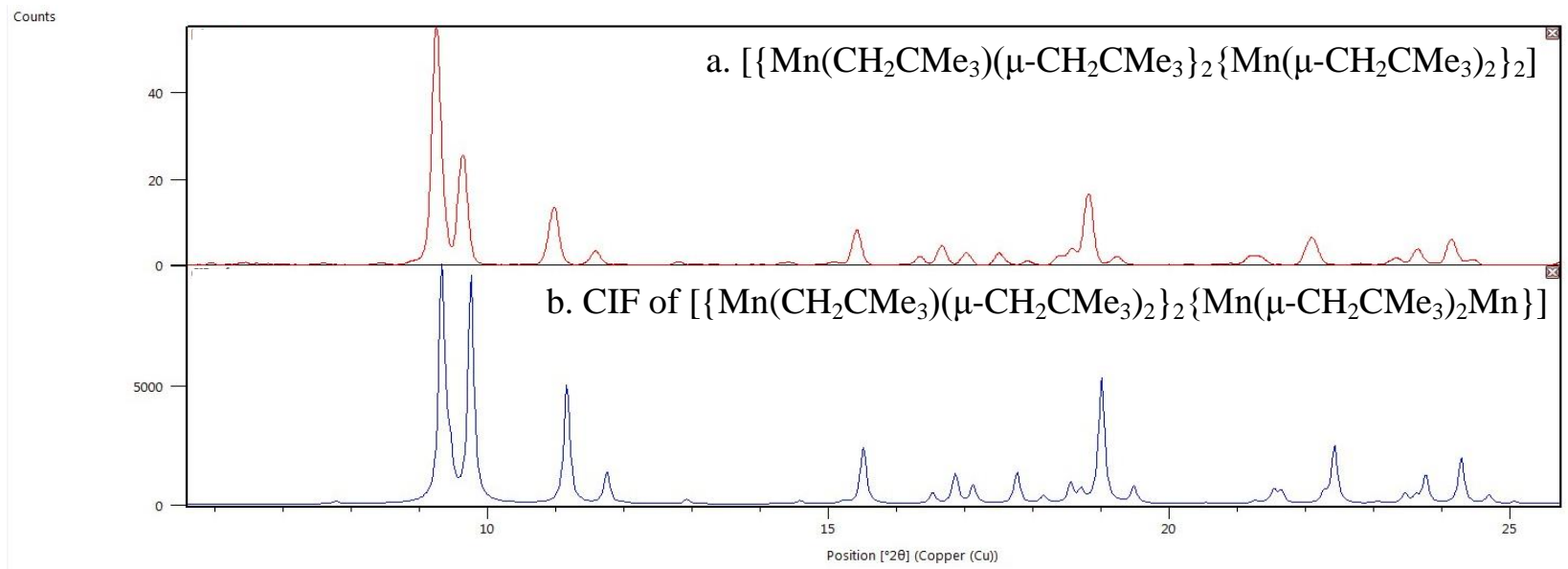

Figure S19: 2D Powder X-ray diffractograms of a. compound 2 (300 K) and b. compound 2 generated from .CIF (100 K) (Cu source $\lambda=1.54056 \AA)$ 
Counts

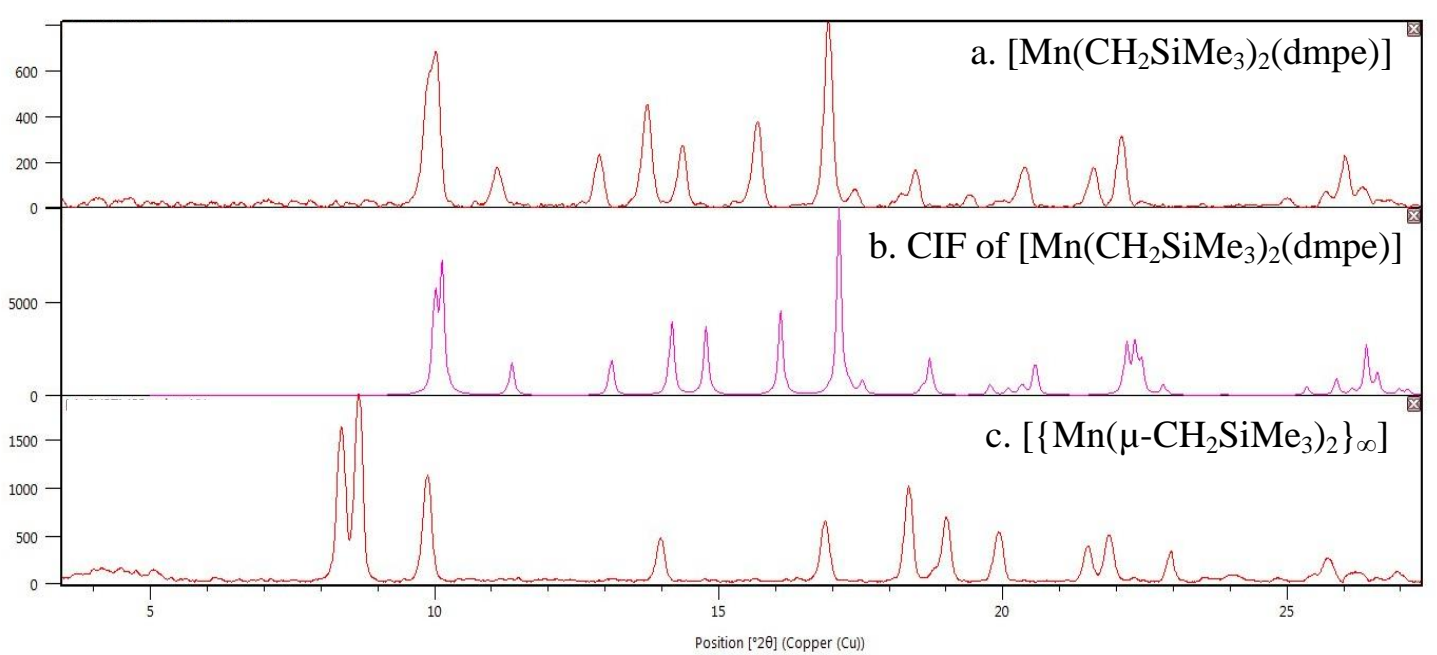

Figure S20: 2D Powder X-ray diffractograms of a. compound 3 (300 K), b. compound 3 generated from .CIF (213 K), and compound 1 (300 K) $(\mathrm{Cu}$ source $\lambda=1.54056 \AA)$

Counts

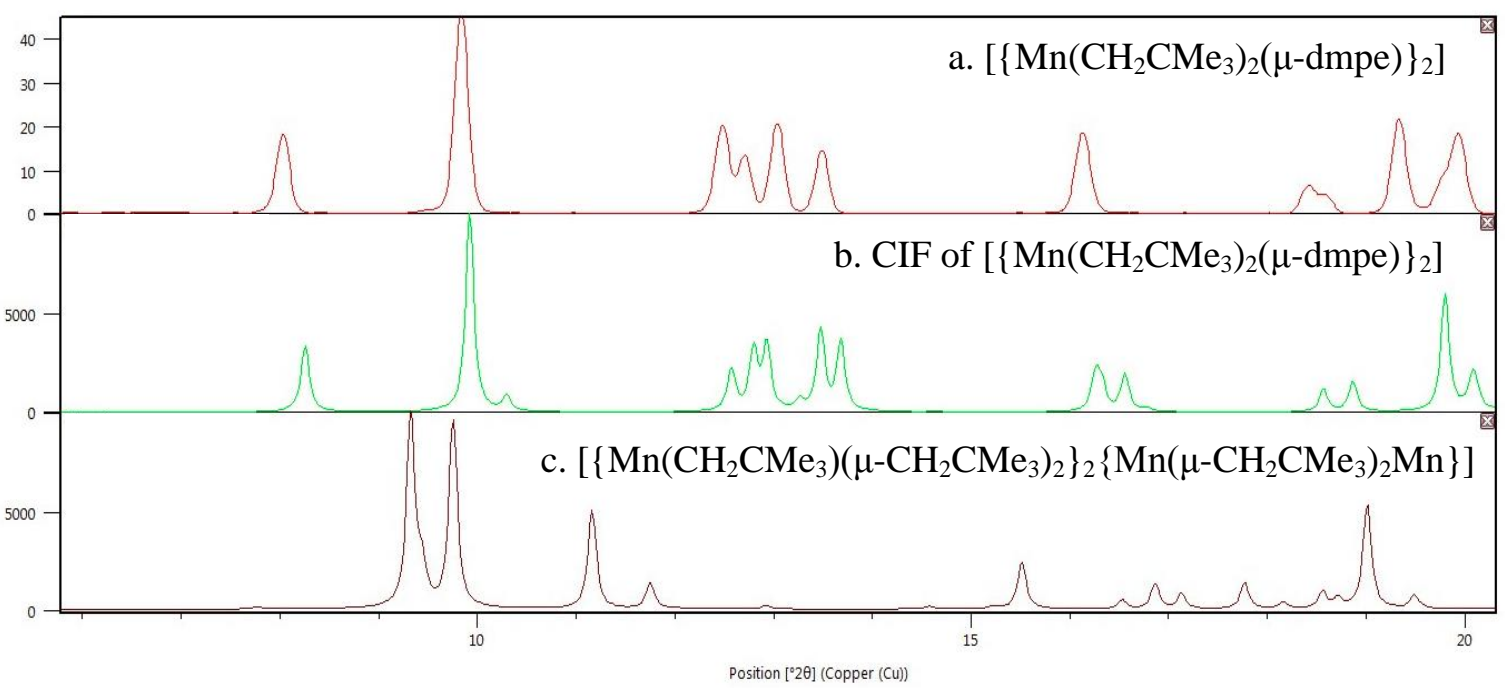

Figure S21: 2D Powder X-ray diffractograms of a. compound 4 (300 K), b. compound 4 generated from .CIF (100 K), and c. compound $2(300 \mathrm{~K})(\mathrm{Cu}$ source $\lambda=1.54056 \AA)$ 


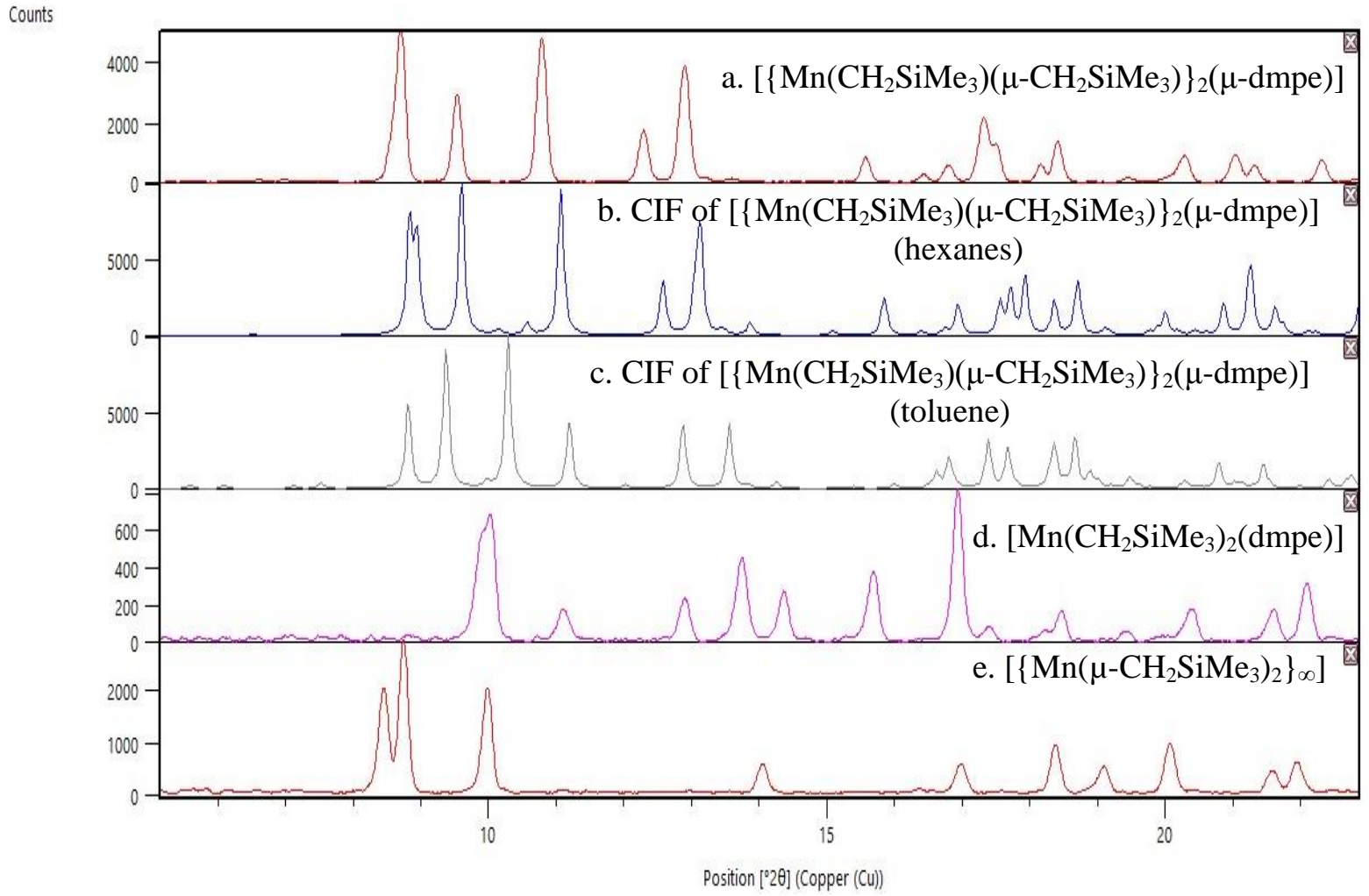

Figure S22: 2D Powder X-ray diffractograms of a. compound 5 (300 K), b. compound 5 generated from .CIF when recrystallized from hexanes $(100 \mathrm{~K})$, c. compound 5 generated from .CIF when recrystallized from toluene (173 K), d. compound 3 (300 $\mathrm{K})$, and e. compound $1(300 \mathrm{~K})(\mathrm{Cu}$ source $\lambda=1.54056 \AA)$ 

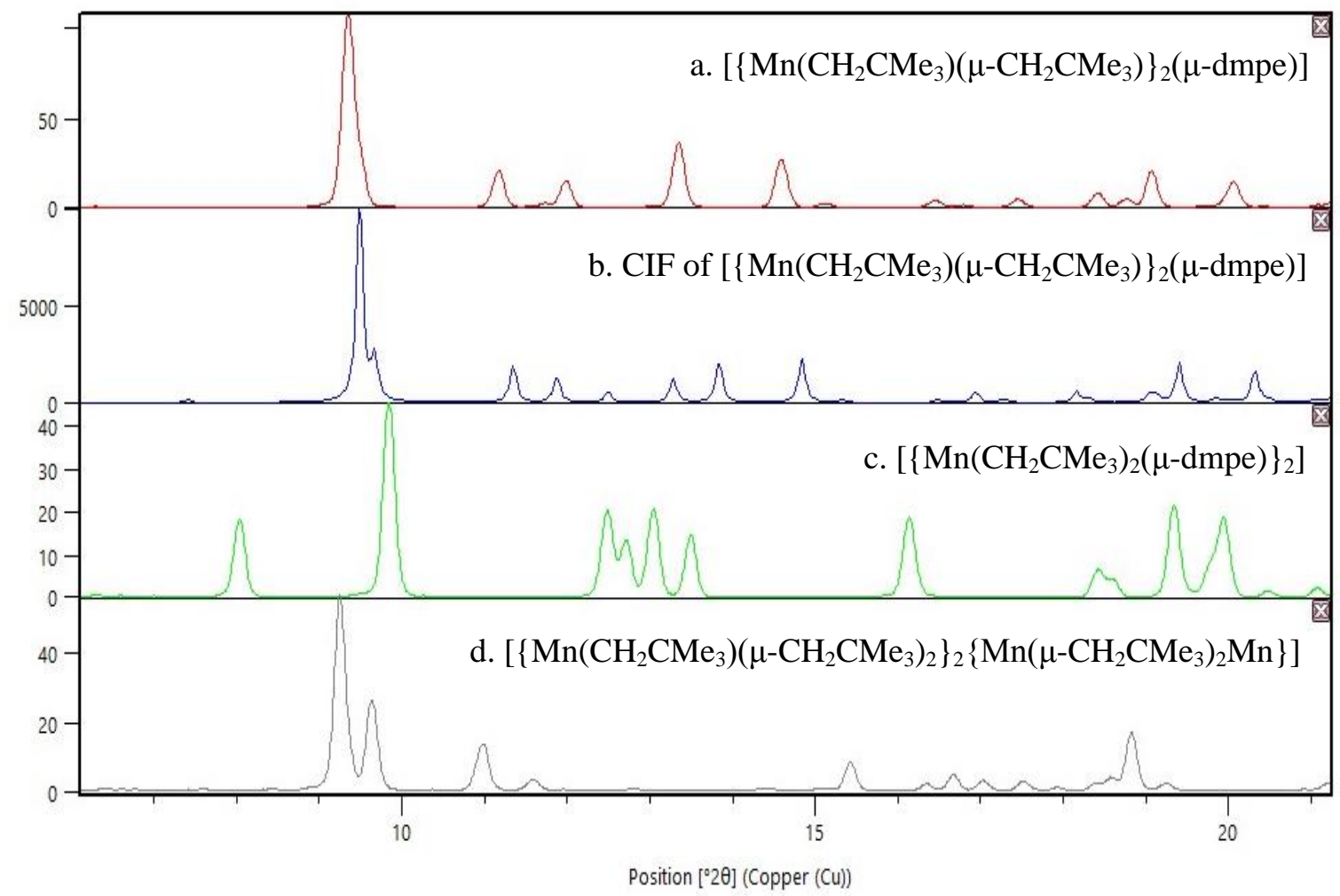

Figure S23: 2D Powder X-ray diffractograms of a. compound 6 (300 K), b. compound 6 generated from .CIF (173 K), c. compound 4 (300 K), and d. compound 2 (300 K) $(\mathrm{Cu}$ source $\lambda=1.54056 \AA)$

Counts

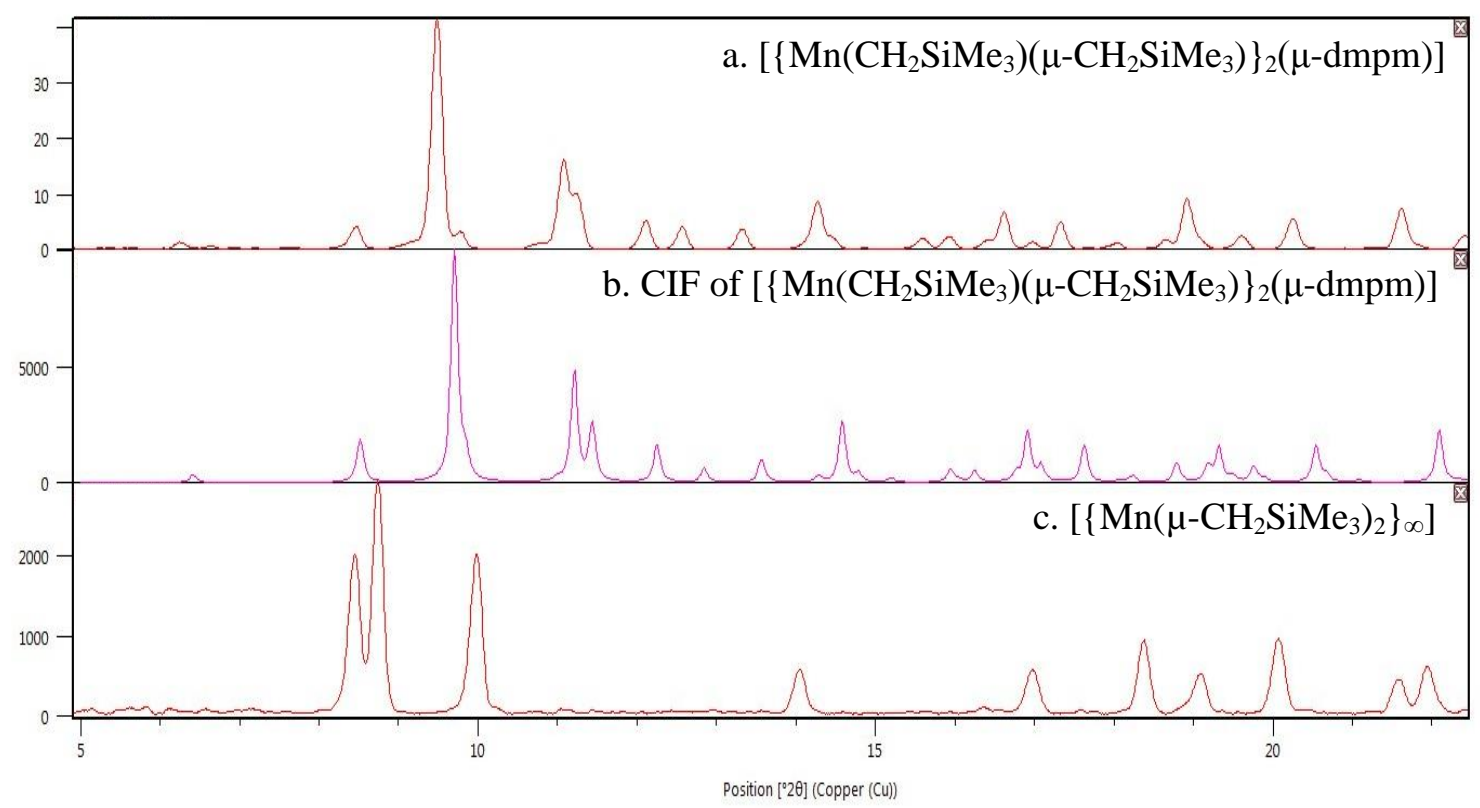

Figure S24: 2D Powder X-ray diffractograms of a. compound 7 (300 K) , b. compound 7 generated from .CIF (100 K), and compound 1 (300 K) (Cu source $\lambda=1.54056 \AA)$ 


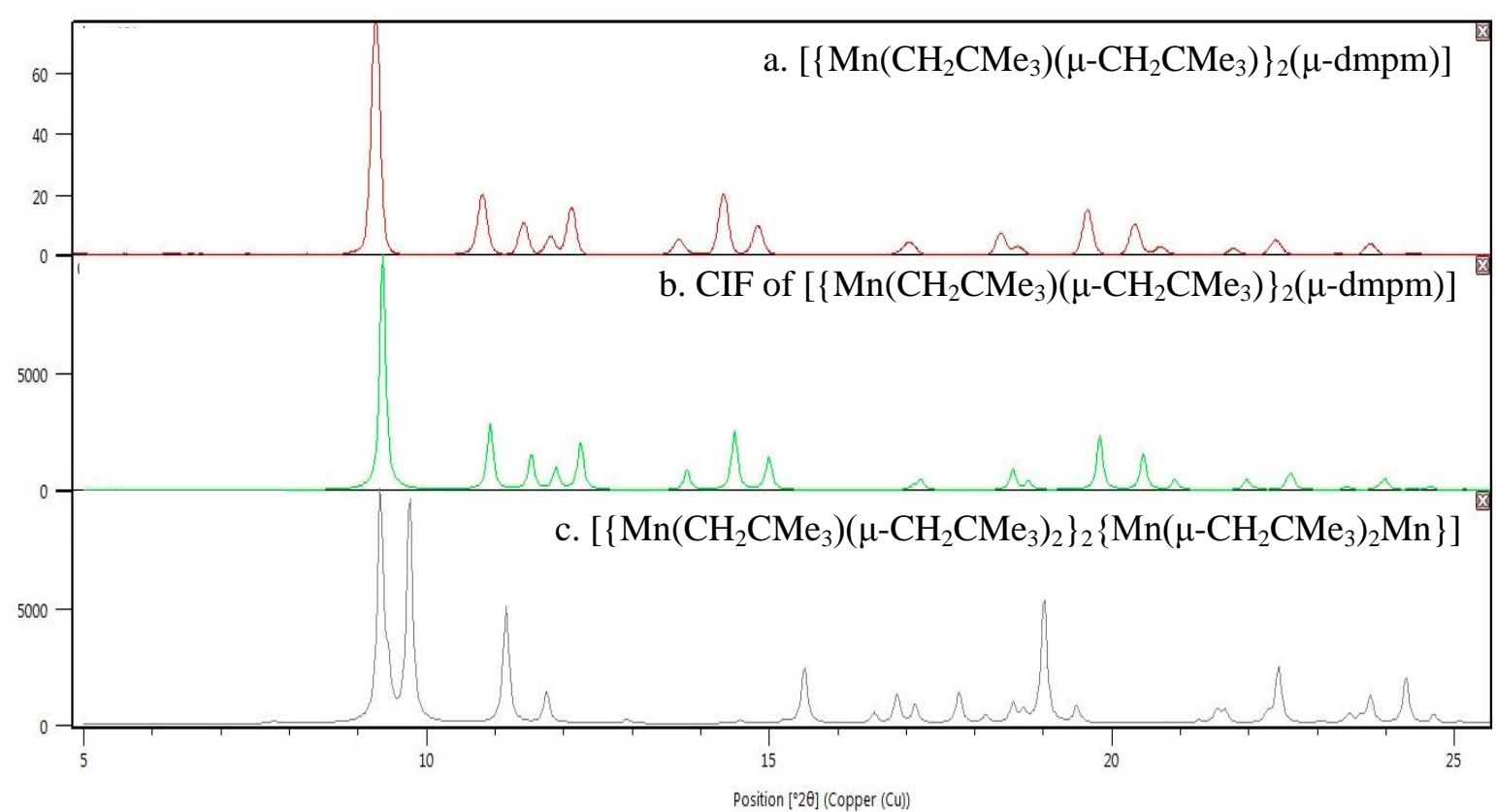

Figure S25: 2D Powder X-ray diffractograms of a. compound 8 (300 K), b. compound 8 generated from .CIF, $(233 \mathrm{~K})$ and compound $2(300 \mathrm{~K})(\mathrm{Cu}$ source $\lambda=1.54056 \AA)$ 

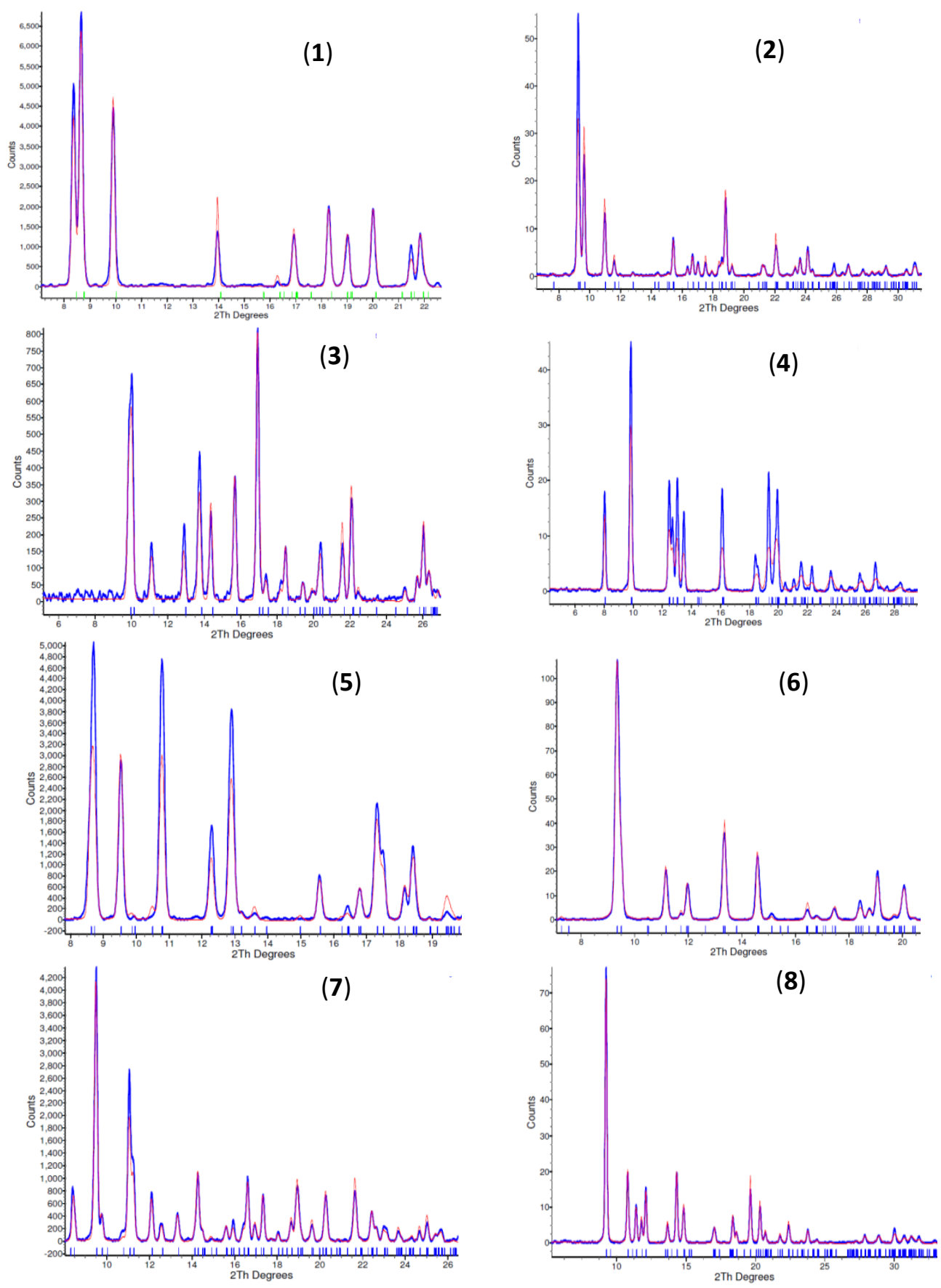

Figure S26: Overlays of 2D powder $x$-ray crystal structures of $\left[\left\{\mathrm{Mn}\left(\mu-\mathrm{CH}_{2} \mathrm{SiMe}\right)_{2}\right\}_{\infty}\right]$ (1) $\left[\left\{\mathrm{Mn}\left(\mathrm{CH}_{2} \mathrm{CMe}_{3}\right)\left(\mu-\mathrm{CH}_{2} \mathrm{CMe}_{3}\right)_{2}\right\}_{2}\left\{\mathrm{Mn}\left(\mu-\mathrm{CH}_{2} \mathrm{CMe}_{3}\right)_{2} \mathrm{Mn}\right\}\right]$ $\left[\mathrm{Mn}\left(\mathrm{CH}_{2} \mathrm{SiMe}_{3}\right)_{2}(\mathrm{dmpe})\right](3),\left[\left\{\mathrm{Mn}\left(\mathrm{CH}_{2} \mathrm{CMe}_{3}\right)_{2}(\mu-\mathrm{dmpe})\right\}_{2}\right](4),\left[\left\{\mathrm{Mn}\left(\mathrm{CH}_{2} \mathrm{SiMe}_{3}\right)(\mu-\right.\right.$ $\left.\left.\mathrm{CH}_{2} \mathrm{SiMe}_{3}\right)\right\}_{2}(\mu$-dmpe) $] \quad(5), \quad\left[\left\{\mathrm{Mn}\left(\mathrm{CH}_{2} \mathrm{CMe}_{3}\right)\left(\mu-\mathrm{CH}_{2} \mathrm{CMe}_{3}\right)\right\}_{2}(\mu\right.$-dmpe) $] \quad$ (6), $\left[\left\{\mathrm{Mn}\left(\mathrm{CH}_{2} \mathrm{SiMe}_{3}\right)\left(\mu-\mathrm{CH}_{2} \mathrm{SiMe}_{3}\right)\right\}_{2}(\mu-\mathrm{dmpm})\right] \quad(7), \quad$ and $\quad\left[\left\{\mathrm{Mn}\left(\mathrm{CH}_{2} \mathrm{CMe}_{3}\right)(\mu-\right.\right.$ $\left.\left.\mathrm{CH}_{2} \mathrm{CMe}_{3}\right)\right\}_{2}(\mu$-dmpm)] (8) collected at $300 \mathrm{~K}$ (blue) over diffractograms generated from single crystal data at lower temperatures with the unit cell parameters relaxed to allow for the expected unit cell expansion to compare to room temperature diffractograms (red) 
ESI 3 SQUID Data Tables for Complexes 2 -8

Complex $2\left(\left[\left\{\mathrm{Mn}\left(\mathrm{CH}_{2} \mathrm{CMe}_{3}\right)\left(\mu-\mathrm{CH}_{2} \mathrm{CMe}_{3}\right)_{2}\right\}_{2}\left\{\mathrm{Mn}\left(\mu-\mathrm{CH}_{2} \mathrm{CMe}_{3}\right)_{2} \mathrm{Mn}\right\}\right]\right)$

Mass (mg): 46.4

Field

(Oe)

100

100

100

100

100

100

100

100

100

100

100

100

100

100

100

100

100

100

100

100

100

100

100

100

100

100

100

100

100

100

100

100

100

100

100

100

100
Mol. Wt. $\left(\mathrm{gmol}^{-1}\right): 788.89$

\begin{tabular}{|c|c|c|c|c|c|c|c|}
\hline K) & EMU & STD & $\begin{array}{c}\text { Chi } \\
\text { (cm3/mol) }\end{array}$ & $\begin{array}{c}\text { dia } \\
(\mathrm{cm} 3 / \mathrm{mol})\end{array}$ & $\begin{array}{l}\text { Cor Chi } \\
(\mathrm{cm} 3 / \mathrm{mol})\end{array}$ & esd & $\begin{array}{c}\text { Chi*T } \\
(\mathrm{cm} 3 \mathrm{~K} / \mathrm{mol}\end{array}$ \\
\hline 300.0 & $3 \mathrm{E}-05$ & $15 \mathrm{E}-08$ & $1.07 \mathrm{E}-02$ & $-5.54 \mathrm{E}-04$ & $1.13 \mathrm{E}-02$ & 1.19E-04 & $3.38 \mathrm{E}+00$ \\
\hline 94.7 & $.31 \mathrm{E}-05$ & $.62 \mathrm{E}-08$ & $1.07 \mathrm{E}-02$ & $-5.54 \mathrm{E}-04$ & $\delta \mathrm{L}-\mathrm{OL}$ & $-1.17 \mathrm{E}-04$ & $3.33 \mathrm{E}+00$ \\
\hline 289.7 & $6.31 \mathrm{E}-05$ & $2.86 \mathrm{E}-08$ & $1.07 \mathrm{E}-02$ & $-5.54 \mathrm{E}-04$ & $1.13 \mathrm{E}-02$ & $-1.17 \mathrm{E}-04$ & $3.27 \mathrm{E}+00$ \\
\hline 284.7 & $6.32 \mathrm{E}-05$ & $94 \mathrm{E}-08$ & $1.07 \mathrm{E}-02$ & $-5.54 \mathrm{E}-04$ & E-02 & $-1.17 \mathrm{E}-04$ & $3.22 \mathrm{E}+00$ \\
\hline 279.7 & $6.33 \mathrm{E}-05$ & $1.49 \mathrm{E}-08$ & $1.08 \mathrm{E}-02$ & $-5.54 \mathrm{E}-04$ & $1.13 \mathrm{E}-02$ & $-1.17 \mathrm{E}-04$ & $3.16 \mathrm{E}+00$ \\
\hline 74.7 & $6.33 \mathrm{E}-05$ & 4.23E-09 & $1.08 \mathrm{E}-02$ & $-5.54 \mathrm{E}-04$ & $1.13 \mathrm{E}-02$ & $-1.17 \mathrm{E}-04$ & $3.11 \mathrm{E}+00$ \\
\hline 269.7 & $6.34 \mathrm{E}-05$ & 4.00E-09 & $1.08 \mathrm{E}-02$ & $-5.54 \mathrm{E}-04$ & $1.13 \mathrm{E}-02$ & $-1.17 \mathrm{E}-04$ & $3.06 \mathrm{E}+00$ \\
\hline 264.7 & $6.35 \mathrm{E}-05$ & 2.19E-08 & $1.08 \mathrm{E}-02$ & $-5.54 \mathrm{E}-04$ & $1.13 \mathrm{E}-02$ & $-1.18 \mathrm{E}-04$ & $3.00 \mathrm{E}+00$ \\
\hline 50 & $251 \mathrm{I}$ & 8 & 2 & -04 & 1 & 1 & $2.95 \mathrm{E}+00$ \\
\hline 254.7 & $6.36 \mathrm{E}-05$ & 9.79E-09 & $1.08 \mathrm{E}-02$ & $-5.54 \mathrm{E}-04$ & $1.14 \mathrm{E}-02$ & $-1.18 \mathrm{E}-04$ & $2.89 \mathrm{E}+00$ \\
\hline 249.7 & $6.36 \mathrm{E}-05$ & E-08 & -02 & -04 & 02 & -04 & $2.84 \mathrm{E}+00$ \\
\hline 244.7 & $6.37 \mathrm{E}-05$ & 8.19E-09 & $1.08 \mathrm{E}-02$ & $-5.54 \mathrm{E}-04$ & $1.14 \mathrm{E}-02$ & $-1.18 \mathrm{E}-04$ & $2.78 \mathrm{E}+00$ \\
\hline 2397 & $643 \mathrm{~F}_{-} 05$ & $103 \mathrm{E}_{-} 06$ & $1.09 \mathrm{E}-02$ & $-5.54 \mathrm{E}-04$ & $1.15 \mathrm{E}-02$ & $-1.19 \mathrm{E}-04$ & $2.75 \mathrm{E}+00$ \\
\hline 234.7 & $6.38 \mathrm{E}-05$ & 6.33E-09 & $1.09 \mathrm{E}-02$ & $-5.54 \mathrm{E}-04$ & E-02 & E-04 & $2.68 \mathrm{E}+00$ \\
\hline 229.7 & $4 \mathrm{E}-05$ & $.61 \mathrm{E}-08$ & $1.09 \mathrm{E}-02$ & $-5.54 \mathrm{E}-04$ & $1.14 \mathrm{E}-02$ & $-1.19 \mathrm{E}-04$ & $2.63 \mathrm{E}+00$ \\
\hline 24.7 & 5 & -09 & $\mathrm{E}-02$ & E-04 & $1.14 \mathrm{E}-02$ & -1 & $2.57 \mathrm{E}+00$ \\
\hline 219.7 & $6.41 \mathrm{E}-05$ & $1.37 \mathrm{E}-08$ & $1.09 \mathrm{E}-02$ & $-5.54 \mathrm{E}-04$ & $1.15 \mathrm{E}-02$ & $-1.19 \mathrm{E}-04$ & $2.52 \mathrm{E}+00$ \\
\hline 214.1 & $6.41 \mathrm{E}-05$ & 7.33E-09 & $1.09 \mathrm{E}-02$ & $-5.54 \mathrm{E}-04$ & $1.15 \mathrm{E}-02$ & $-1.19 \mathrm{E}-04$ & $2.46 \mathrm{E}+00$ \\
\hline 09.7 & $6.42 \mathrm{E}-05$ & 00 & $1.09 \mathrm{E}-02$ & $-5.54 \mathrm{E}-04$ & $1.15 \mathrm{E}-02$ & E-04 & $2.41 \mathrm{E}+00$ \\
\hline 204.7 & $6.42 \mathrm{E}-05$ & 3.77E-09 & $1.09 \mathrm{E}-02$ & $-5.54 \mathrm{E}-04$ & $1.15 \mathrm{E}-02$ & $-1.19 \mathrm{E}-04$ & $2.35 \mathrm{E}+00$ \\
\hline 199.7 & $6.43 \mathrm{E}-05$ & -09 & $1.09 \mathrm{E}-02$ & $-5.54 \mathrm{E}-04$ & E-02 & -04 & $2.29 \mathrm{E}+00$ \\
\hline 94.7 & $6.43 \mathrm{E}-05$ & 3.8 & E-02 & $-5.54 \mathrm{E}-04$ & E-02 & $-1.19 \mathrm{E}-04$ & $2.24 \mathrm{E}+00$ \\
\hline 189.8 & $6.44 \mathrm{E}-05$ & $3.96 \mathrm{E}-09$ & $1.09 \mathrm{E}-02$ & $-5.54 \mathrm{E}-04$ & E-02 & $-1.19 \mathrm{E}-04$ & $2.18 \mathrm{E}+00$ \\
\hline 184.8 & $6.44 \mathrm{E}-05$ & 6.11E-09 & $1.10 \mathrm{E}-02$ & $-5.54 \mathrm{E}-04$ & & & $2.13 \mathrm{E}+00$ \\
\hline 179.8 & $6.45 \mathrm{E}-05$ & $1.74 \mathrm{E}-08$ & $1.10 \mathrm{E}-02$ & $-5.54 \mathrm{E}-04$ & $1.15 \mathrm{E}-02$ & $-1.19 \mathrm{E}-04$ & $2.07 \mathrm{E}+00$ \\
\hline 74.8 & 05 & -09 & -02 & -5.5 & 1. & 4 & $2.01 \mathrm{E}+00$ \\
\hline 169.8 & $6.45 \mathrm{E}-05$ & $2.95 \mathrm{E}-08$ & $1.10 \mathrm{E}-02$ & & & & $1.96 \mathrm{E}+00$ \\
\hline 164.8 & $6.45 \mathrm{E}-05$ & 1.69E-09 & $1.10 \mathrm{E}-02$ & $-5.54 \mathrm{E}-04$ & $1.15 \mathrm{E}-02$ & $-1.19 \mathrm{E}-04$ & $1.90 \mathrm{E}+00$ \\
\hline 1508 & 05 & -09 & $\mathrm{E}-02$ & $-5.54 \mathrm{E}-04$ & E-02 & -1 . & $1.84 \mathrm{E}+00$ \\
\hline 154.8 & $6.46 \mathrm{E}-05$ & $1.13 \mathrm{E}-08$ & $1.10 \mathrm{E}-02$ & $-5.54 \mathrm{E}-04$ & $1.15 \mathrm{E}-02$ & $-1.20 \mathrm{E}-04$ & $1.79 \mathrm{E}+00$ \\
\hline 149.9 & $6.47 \mathrm{E}-05$ & $2.10 \mathrm{E}-08$ & $1.10 \mathrm{E}-02$ & $-5.54 \mathrm{E}-04$ & $1.16 \mathrm{E}-02$ & $-1.20 \mathrm{E}-04$ & $1.73 \mathrm{E}+00$ \\
\hline 144.9 & $6.48 \mathrm{E}-05$ & $2.78 \mathrm{E}-08$ & $1.10 \mathrm{E}-02$ & $-5.54 \mathrm{E}-04$ & $1.16 \mathrm{E}-02$ & $-1.20 \mathrm{E}-04$ & $1.68 \mathrm{E}+00$ \\
\hline 139.9 & $6.49 \mathrm{E}-05$ & $2.91 \mathrm{E}-10$ & $1.10 \mathrm{E}-02$ & $-5.54 \mathrm{E}-04$ & $1.16 \mathrm{E}-02$ & $-1.20 \mathrm{E}-04$ & $1.62 \mathrm{E}+00$ \\
\hline 134.9 & $6.49 \mathrm{E}-05$ & $1.05 \mathrm{E}-08$ & $1.10 \mathrm{E}-02$ & $-5.54 \mathrm{E}-04$ & $1.16 \mathrm{E}-02$ & $-1.20 \mathrm{E}-04$ & $1.56 \mathrm{E}+00$ \\
\hline 129.9 & $6.5 \mathrm{E}-05$ & 7.26E-09 & $1.10 \mathrm{E}-02$ & $-5.54 \mathrm{E}-04$ & $1.16 \mathrm{E}-02$ & $-1.20 \mathrm{E}-04$ & $1.51 \mathrm{E}+00$ \\
\hline 124.9 & $6.5 \mathrm{E}-05$ & $1.11 \mathrm{E}-08$ & $1.11 \mathrm{E}-02$ & $-5.54 \mathrm{E}-04$ & $1.16 \mathrm{E}-02$ & $-1.20 \mathrm{E}-04$ & $1.45 \mathrm{E}+00$ \\
\hline 120.0 & $6.51 \mathrm{E}-05$ & $1.75 \mathrm{E}-08$ & $1.11 \mathrm{E}-02$ & $-5.54 \mathrm{E}-04$ & $1.16 \mathrm{E}-02$ & $-1.21 \mathrm{E}-04$ & $1.39 \mathrm{E}+00$ \\
\hline
\end{tabular}




\begin{tabular}{|c|c|c|c|c|c|c|c|c|}
\hline 100 & 115.0 & $6.52 \mathrm{E}-05$ & $1.43 \mathrm{E}-08$ & $1.11 \mathrm{E}-02$ & $-5.54 \mathrm{E}-04$ & E- 02 & $-1.21 \mathrm{E}-04$ & $4 \mathrm{E}+00$ \\
\hline 100 & 110.0 & $6.52 \mathrm{E}-05$ & $1.20 \mathrm{E}-08$ & $1.11 \mathrm{E}-02$ & $-5.54 \mathrm{E}-04$ & $1.16 \mathrm{E}-02$ & $-1.21 \mathrm{E}-04$ & $1.28 \mathrm{E}+00$ \\
\hline 100 & 105.0 & $6.52 \mathrm{E}-05$ & $2.07 \mathrm{E}-08$ & $1.11 \mathrm{E}-02$ & $-5.54 \mathrm{E}-04$ & $1.16 \mathrm{E}-02$ & $-1.21 \mathrm{E}-04$ & $1.22 \mathrm{E}+00$ \\
\hline 100 & 100.0 & $6.52 \mathrm{E}-05$ & $1.26 \mathrm{E}-08$ & $1.11 \mathrm{E}-02$ & $-5.54 \mathrm{E}-04$ & $1.16 \mathrm{E}-02$ & $-1.21 \mathrm{E}-04$ & $1.16 \mathrm{E}+00$ \\
\hline 100 & 98.9 & 6.53E-05 & $3.00 \mathrm{E}-08$ & $1.11 \mathrm{E}-02$ & $-5.54 \mathrm{E}-04$ & $1.17 \mathrm{E}-02$ & $-1.21 \mathrm{E}-04$ & $1.15 \mathrm{E}+00$ \\
\hline 100 & 97.9 & $6.53 \mathrm{E}-05$ & $1.23 \mathrm{E}-08$ & $1.11 \mathrm{E}-02$ & $-5.54 \mathrm{E}-04$ & $1.17 \mathrm{E}-02$ & $-1.21 \mathrm{E}-04$ & $1.14 \mathrm{E}+00$ \\
\hline 100 & 96.9 & $6.53 \mathrm{E}-05$ & 4.72E-09 & $1.11 \mathrm{E}-02$ & $-5.54 \mathrm{E}-04$ & $1.17 \mathrm{E}-02$ & $-1.21 \mathrm{E}-04$ & $1.13 \mathrm{E}+00$ \\
\hline 100 & 95.9 & $6.53 \mathrm{E}-05$ & 5.09E-09 & $1.11 \mathrm{E}-02$ & $-5.54 \mathrm{E}-04$ & $1.17 \mathrm{E}-02$ & $-1.21 \mathrm{E}-04$ & $1.12 \mathrm{E}+00$ \\
\hline 100 & 94.9 & $6.54 \mathrm{E}-05$ & 5.85E-09 & $1.11 \mathrm{E}-02$ & $-5.54 \mathrm{E}-04$ & $1.17 \mathrm{E}-02$ & $-1.21 \mathrm{E}-04$ & $1.11 \mathrm{E}+00$ \\
\hline 100 & 93.9 & $6.54 \mathrm{E}-05$ & $1.11 \mathrm{E}-08$ & $1.11 \mathrm{E}-02$ & $-5.54 \mathrm{E}-04$ & $1.17 \mathrm{E}-02$ & $-1.21 \mathrm{E}-04$ & $1.10 \mathrm{E}+00$ \\
\hline 100 & 92.9 & $6.54 \mathrm{E}-05$ & $1.31 \mathrm{E}-08$ & $1.11 \mathrm{E}-02$ & $-5.54 \mathrm{E}-04$ & $1.17 \mathrm{E}-02$ & $-1.21 \mathrm{E}-04$ & $1.08 \mathrm{E}+00$ \\
\hline 100 & 91.9 & $6.54 \mathrm{E}-05$ & 4.28E-09 & $1.11 \mathrm{E}-02$ & $-5.54 \mathrm{E}-04$ & $1.17 \mathrm{E}-02$ & $-1.21 \mathrm{E}-04$ & $1.07 \mathrm{E}+00$ \\
\hline 100 & 90.9 & $6.54 \mathrm{E}-05$ & 4.98E-09 & $1.11 \mathrm{E}-02$ & $-5.54 \mathrm{E}-04$ & $1.17 \mathrm{E}-02$ & $-1.21 \mathrm{E}-04$ & $1.06 \mathrm{E}+00$ \\
\hline 100 & 89.9 & $6.54 \mathrm{E}-05$ & $6.09 \mathrm{E}-09$ & $1.11 \mathrm{E}-02$ & $-5.54 \mathrm{E}-04$ & $1.17 \mathrm{E}-02$ & $-1.21 \mathrm{E}-04$ & $1.05 \mathrm{E}+00$ \\
\hline 100 & 88.9 & 6.54E-05 & $6.35 \mathrm{E}-10$ & $1.11 \mathrm{E}-02$ & $-5.54 \mathrm{E}-04$ & $1.17 \mathrm{E}-02$ & $-1.21 \mathrm{E}-04$ & $1.04 \mathrm{E}+00$ \\
\hline 100 & 87.9 & $6.53 \mathrm{E}-05$ & $2.45 \mathrm{E}-08$ & $1.11 \mathrm{E}-02$ & $-5.54 \mathrm{E}-04$ & $1.17 \mathrm{E}-02$ & $-1.21 \mathrm{E}-04$ & $1.03 \mathrm{E}+00$ \\
\hline 100 & 86.9 & 6.53E-05 & 5.59E-09 & $1.11 \mathrm{E}-02$ & $-5.54 \mathrm{E}-04$ & $1.17 \mathrm{E}-02$ & $-1.21 \mathrm{E}-04$ & $1.01 \mathrm{E}+00$ \\
\hline 100 & 85.9 & $6.53 \mathrm{E}-05$ & $1.06 \mathrm{E}-08$ & $1.11 \mathrm{E}-02$ & $-5.54 \mathrm{E}-04$ & $1.17 \mathrm{E}-02$ & $-1.21 \mathrm{E}-04$ & $1.00 \mathrm{E}+00$ \\
\hline 100 & 84.9 & $6.53 \mathrm{E}-05$ & 8.14E-09 & $1.11 \mathrm{E}-02$ & $-5.54 \mathrm{E}-04$ & $1.17 \mathrm{E}-02$ & $-1.21 \mathrm{E}-04$ & $9.90 \mathrm{E}-01$ \\
\hline 100 & 83.9 & $6.53 \mathrm{E}-05$ & $6.24 \mathrm{E}-09$ & $1.11 \mathrm{E}-02$ & $-5.54 \mathrm{E}-04$ & $1.17 \mathrm{E}-02$ & $-1.21 \mathrm{E}-04$ & $9.78 \mathrm{E}-01$ \\
\hline 100 & 82.9 & $6.53 \mathrm{E}-05$ & $1.46 \mathrm{E}-08$ & $1.11 \mathrm{E}-02$ & $-5.54 \mathrm{E}-04$ & $1.17 \mathrm{E}-02$ & $-1.21 \mathrm{E}-04$ & $9.66 \mathrm{E}-01$ \\
\hline 100 & 81.9 & $6.52 \mathrm{E}-05$ & $1.20 \mathrm{E}-08$ & $1.11 \mathrm{E}-02$ & $-5.54 \mathrm{E}-04$ & $1.16 \mathrm{E}-02$ & $-1.21 \mathrm{E}-04$ & $9.54 \mathrm{E}-01$ \\
\hline 100 & 80.9 & $6.52 \mathrm{E}-05$ & 4.81E-09 & $1.11 \mathrm{E}-02$ & $-5.54 \mathrm{E}-04$ & $1.16 \mathrm{E}-02$ & $-1.21 \mathrm{E}-04$ & $9.42 \mathrm{E}-01$ \\
\hline 100 & 80.0 & $6.52 \mathrm{E}-05$ & $1.57 \mathrm{E}-08$ & $1.11 \mathrm{E}-02$ & $-5.54 \mathrm{E}-04$ & $1.16 \mathrm{E}-02$ & $-1.21 \mathrm{E}-04$ & $9.30 \mathrm{E}-01$ \\
\hline 100 & 79.0 & $6.52 \mathrm{E}-05$ & $6.84 \mathrm{E}-09$ & $1.11 \mathrm{E}-02$ & $-5.54 \mathrm{E}-04$ & $1.16 \mathrm{E}-02$ & $-1.21 \mathrm{E}-04$ & $9.18 \mathrm{E}-01$ \\
\hline 100 & 78.0 & $6.52 \mathrm{E}-05$ & 4.95E-09 & $1.11 \mathrm{E}-02$ & $-5.54 \mathrm{E}-04$ & $1.16 \mathrm{E}-02$ & $-1.21 \mathrm{E}-04$ & $9.07 \mathrm{E}-01$ \\
\hline 100 & 77.0 & $6.51 \mathrm{E}-05$ & 7.23E-09 & $1.11 \mathrm{E}-02$ & $-5.54 \mathrm{E}-04$ & $1.16 \mathrm{E}-02$ & $-1.21 \mathrm{E}-04$ & 8.95E-01 \\
\hline 100 & 76.0 & $6.51 \mathrm{E}-05$ & $2.55 \mathrm{E}-08$ & $1.11 \mathrm{E}-02$ & $-5.54 \mathrm{E}-04$ & $1.16 \mathrm{E}-02$ & $-1.21 \mathrm{E}-04$ & $8.83 \mathrm{E}-01$ \\
\hline 100 & 75.0 & $6.5 \mathrm{E}-05$ & 1.63E-09 & $1.11 \mathrm{E}-02$ & $-5.54 \mathrm{E}-04$ & $1.16 \mathrm{E}-02$ & $-1.20 \mathrm{E}-04$ & 8.70E-01 \\
\hline 100 & 74.0 & $6.5 \mathrm{E}-05$ & 3.93E-09 & $1.10 \mathrm{E}-02$ & $-5.54 \mathrm{E}-04$ & $1.16 \mathrm{E}-02$ & $-1.20 \mathrm{E}-04$ & $8.58 \mathrm{E}-01$ \\
\hline 100 & 73.0 & $6.5 \mathrm{E}-05$ & $1.29 \mathrm{E}-08$ & $1.10 \mathrm{E}-02$ & $-5.54 \mathrm{E}-04$ & $1.16 \mathrm{E}-02$ & $-1.20 \mathrm{E}-04$ & $8.46 \mathrm{E}-01$ \\
\hline 100 & 72.0 & $6.49 \mathrm{E}-05$ & $1.74 \mathrm{E}-08$ & $1.10 \mathrm{E}-02$ & $-5.54 \mathrm{E}-04$ & $1.16 \mathrm{E}-02$ & $-1.20 \mathrm{E}-04$ & 8.35E-01 \\
\hline 100 & 71.0 & $6.49 \mathrm{E}-05$ & $2.71 \mathrm{E}-08$ & $1.10 \mathrm{E}-02$ & $-5.54 \mathrm{E}-04$ & $1.16 \mathrm{E}-02$ & $-1.20 \mathrm{E}-04$ & $8.23 \mathrm{E}-01$ \\
\hline 100 & 70.0 & $6.48 \mathrm{E}-05$ & $1.25 \mathrm{E}-08$ & $1.10 \mathrm{E}-02$ & $-5.54 \mathrm{E}-04$ & $1.16 \mathrm{E}-02$ & $-1.20 \mathrm{E}-04$ & 8.11E-01 \\
\hline 100 & 69.0 & $6.48 \mathrm{E}-05$ & $3.98 \mathrm{E}-08$ & $1.10 \mathrm{E}-02$ & $-5.54 \mathrm{E}-04$ & $1.16 \mathrm{E}-02$ & $-1.20 \mathrm{E}-04$ & 7.99E-01 \\
\hline 100 & 68.0 & $6.48 \mathrm{E}-05$ & $1.42 \mathrm{E}-08$ & $1.10 \mathrm{E}-02$ & $-5.54 \mathrm{E}-04$ & $1.16 \mathrm{E}-02$ & $-1.20 \mathrm{E}-04$ & $7.88 \mathrm{E}-01$ \\
\hline 100 & 67.0 & $6.48 \mathrm{E}-05$ & $2.34 \mathrm{E}-08$ & $1.10 \mathrm{E}-02$ & $-5.54 \mathrm{E}-04$ & $1.16 \mathrm{E}-02$ & $-1.20 \mathrm{E}-04$ & 7.76E-01 \\
\hline 100 & 66.0 & $6.48 \mathrm{E}-05$ & $1.80 \mathrm{E}-08$ & $1.10 \mathrm{E}-02$ & $-5.54 \mathrm{E}-04$ & $1.16 \mathrm{E}-02$ & $-1.20 \mathrm{E}-04$ & 7.64E-01 \\
\hline 100 & 65.0 & $6.48 \mathrm{E}-05$ & $1.49 \mathrm{E}-08$ & $1.10 \mathrm{E}-02$ & $-5.54 \mathrm{E}-04$ & $1.16 \mathrm{E}-02$ & $-1.20 \mathrm{E}-04$ & 7.53E-01 \\
\hline 100 & 64.0 & $6.47 \mathrm{E}-05$ & 7.88E-09 & $1.10 \mathrm{E}-02$ & $-5.54 \mathrm{E}-04$ & $1.16 \mathrm{E}-02$ & $-1.20 \mathrm{E}-04$ & 7.40E-01 \\
\hline 100 & 63.0 & $6.46 \mathrm{E}-05$ & $1.23 \mathrm{E}-08$ & $1.10 \mathrm{E}-02$ & $-5.54 \mathrm{E}-04$ & $1.15 \mathrm{E}-02$ & $-1.20 \mathrm{E}-04$ & $7.28 \mathrm{E}-01$ \\
\hline 100 & 62.0 & $6.46 \mathrm{E}-05$ & $1.30 \mathrm{E}-08$ & $1.10 \mathrm{E}-02$ & $-5.54 \mathrm{E}-04$ & $1.15 \mathrm{E}-02$ & $-1.20 \mathrm{E}-04$ & 7.16E-01 \\
\hline 100 & 61.0 & $6.46 \mathrm{E}-05$ & $1.17 \mathrm{E}-08$ & $1.10 \mathrm{E}-02$ & $-5.54 \mathrm{E}-04$ & $1.15 \mathrm{E}-02$ & $-1.20 \mathrm{E}-04$ & 7.04E-01 \\
\hline
\end{tabular}




\begin{tabular}{|c|c|c|c|c|c|c|c|c|}
\hline 100 & 60.0 & $6.45 \mathrm{E}-05$ & 2.12E-08 & $1.10 \mathrm{E}-02$ & $-5.54 \mathrm{E}-04$ & $1.15 \mathrm{E}-02$ & $-1.19 \mathrm{E}-04$ & $6.92 \mathrm{E}-01$ \\
\hline 100 & 59.0 & $45 \mathrm{E}-05$ & 3.57E-09 & $1.10 \mathrm{E}-02$ & $-5.54 \mathrm{E}-04$ & $1.15 \mathrm{E}-02$ & $-1.19 \mathrm{E}-04$ & $6.80 \mathrm{E}-01$ \\
\hline 100 & 58.0 & $6.44 \mathrm{E}-05$ & 2.34E-09 & $1.09 \mathrm{E}-02$ & $-5.54 \mathrm{E}-04$ & $1.15 \mathrm{E}-02$ & $-1.19 \mathrm{E}-04$ & $6.68 \mathrm{E}-01$ \\
\hline 100 & 57.0 & $6.43 \mathrm{E}-05$ & 7.38E-09 & $1.09 \mathrm{E}-02$ & $-5.54 \mathrm{E}-04$ & $1.15 \mathrm{E}-02$ & $-1.19 \mathrm{E}-04$ & $6.55 \mathrm{E}-01$ \\
\hline 100 & 56.0 & $6.42 \mathrm{E}-05$ & $1.43 \mathrm{E}-08$ & $1.09 \mathrm{E}-02$ & $-5.54 \mathrm{E}-04$ & $1.15 \mathrm{E}-02$ & $-1.19 \mathrm{E}-04$ & 6.43E-01 \\
\hline 100 & 55.0 & $6.41 \mathrm{E}-05$ & $3.28 \mathrm{E}-09$ & $1.09 \mathrm{E}-02$ & $-5.54 \mathrm{E}-04$ & $1.15 \mathrm{E}-02$ & $-1.19 \mathrm{E}-04$ & $6.31 \mathrm{E}-01$ \\
\hline 100 & 54.0 & 6.4E-05 & $1.06 \mathrm{E}-08$ & $1.09 \mathrm{E}-02$ & $-5.54 \mathrm{E}-04$ & $1.14 \mathrm{E}-02$ & $-1.19 \mathrm{E}-04$ & $6.18 \mathrm{E}-01$ \\
\hline 100 & 53.1 & $6.39 \mathrm{E}-05$ & $1.10 \mathrm{E}-08$ & $1.09 \mathrm{E}-02$ & $-5.54 \mathrm{E}-04$ & $1.14 \mathrm{E}-02$ & $-1.18 \mathrm{E}-04$ & $6.06 \mathrm{E}-01$ \\
\hline 100 & 52.0 & $6.37 \mathrm{E}-05$ & 6.31E-09 & $1.08 \mathrm{E}-02$ & $-5.54 \mathrm{E}-04$ & $1.14 \mathrm{E}-02$ & $-1.18 \mathrm{E}-04$ & 5.93E-01 \\
\hline 100 & 51.0 & $6.36 \mathrm{E}-05$ & 4.12E-09 & $1.08 \mathrm{E}-02$ & $-5.54 \mathrm{E}-04$ & $1.14 \mathrm{E}-02$ & $-1.18 \mathrm{E}-04$ & $5.80 \mathrm{E}-01$ \\
\hline 100 & 50.0 & $6.35 \mathrm{E}-05$ & $1.31 \mathrm{E}-08$ & $1.08 \mathrm{E}-02$ & $-5.54 \mathrm{E}-04$ & $1.13 \mathrm{E}-02$ & $-1.18 \mathrm{E}-04$ & $5.68 \mathrm{E}-01$ \\
\hline 100 & 49.0 & $6.33 \mathrm{E}-05$ & 7.36E-09 & $1.08 \mathrm{E}-02$ & $-5.54 \mathrm{E}-04$ & $1.13 \mathrm{E}-02$ & $-1.17 \mathrm{E}-04$ & $5.55 \mathrm{E}-01$ \\
\hline 100 & 48.0 & $6.32 \mathrm{E}-05$ & 8.17E-09 & $1.07 \mathrm{E}-02$ & $-5.54 \mathrm{E}-04$ & $1.13 \mathrm{E}-02$ & $-1.17 \mathrm{E}-04$ & 5.43E-01 \\
\hline 100 & 47.0 & 6.3E-05 & $1.50 \mathrm{E}-08$ & 1.07E-02 & $-5.54 \mathrm{E}-04$ & $1.13 \mathrm{E}-02$ & $-1.17 \mathrm{E}-04$ & $5.30 \mathrm{E}-01$ \\
\hline 100 & 46.0 & $6.29 \mathrm{E}-05$ & $9.36 \mathrm{E}-09$ & $1.07 \mathrm{E}-02$ & $-5.54 \mathrm{E}-04$ & $1.12 \mathrm{E}-02$ & $-1.16 \mathrm{E}-04$ & $5.18 \mathrm{E}-01$ \\
\hline 100 & 45.0 & $6.27 \mathrm{E}-05$ & $1.37 \mathrm{E}-08$ & $1.07 \mathrm{E}-02$ & $-5.54 \mathrm{E}-04$ & $1.12 \mathrm{E}-02$ & $-1.16 \mathrm{E}-04$ & $5.05 \mathrm{E}-01$ \\
\hline 100 & 44.0 & $6.24 \mathrm{E}-05$ & $2.60 \mathrm{E}-08$ & $1.06 \mathrm{E}-02$ & $-5.54 \mathrm{E}-04$ & $1.12 \mathrm{E}-02$ & $-1.16 \mathrm{E}-04$ & 4.92E-01 \\
\hline 100 & 43.0 & $6.23 \mathrm{E}-05$ & $8.75 \mathrm{E}-09$ & $1.06 \mathrm{E}-02$ & $-5.54 \mathrm{E}-04$ & $1.11 \mathrm{E}-02$ & $-1.15 \mathrm{E}-04$ & 4.79E-01 \\
\hline 100 & 42.0 & $6.21 \mathrm{E}-05$ & $3.03 \mathrm{E}-08$ & $1.06 \mathrm{E}-02$ & $-5.54 \mathrm{E}-04$ & $1.11 \mathrm{E}-02$ & $-1.15 \mathrm{E}-04$ & 4.67E-01 \\
\hline 100 & 41.0 & $6.18 \mathrm{E}-05$ & $2.27 \mathrm{E}-08$ & $1.05 \mathrm{E}-02$ & $-5.54 \mathrm{E}-04$ & $1.11 \mathrm{E}-02$ & $-1.14 \mathrm{E}-04$ & 4.54E-01 \\
\hline 100 & 40.0 & $6.15 \mathrm{E}-05$ & $2.01 \mathrm{E}-08$ & $1.05 \mathrm{E}-02$ & $-5.54 \mathrm{E}-04$ & $1.10 \mathrm{E}-02$ & $-1.14 \mathrm{E}-04$ & 4.40E-01 \\
\hline 100 & 39.0 & $6.12 \mathrm{E}-05$ & $3.25 \mathrm{E}-09$ & $1.04 \mathrm{E}-02$ & $-5.54 \mathrm{E}-04$ & $1.10 \mathrm{E}-02$ & $-1.13 \mathrm{E}-04$ & 4.28E-01 \\
\hline 100 & 38.0 & $6.09 \mathrm{E}-05$ & $1.30 \mathrm{E}-08$ & $1.04 \mathrm{E}-02$ & $-5.54 \mathrm{E}-04$ & $1.09 \mathrm{E}-02$ & $-1.13 \mathrm{E}-04$ & 4.15E-01 \\
\hline 100 & 37.0 & $6.06 \mathrm{E}-05$ & $2.45 \mathrm{E}-08$ & $1.03 \mathrm{E}-02$ & $-5.54 \mathrm{E}-04$ & $1.09 \mathrm{E}-02$ & $-1.12 \mathrm{E}-04$ & 4.02E-01 \\
\hline 100 & 36.0 & $6.01 \mathrm{E}-05$ & 9.03E-09 & $1.02 \mathrm{E}-02$ & $-5.54 \mathrm{E}-04$ & $1.08 \mathrm{E}-02$ & $-1.11 \mathrm{E}-04$ & $3.88 \mathrm{E}-01$ \\
\hline 100 & 35.0 & 5.97E-05 & $1.41 \mathrm{E}-08$ & $1.02 \mathrm{E}-02$ & $-5.54 \mathrm{E}-04$ & $1.07 \mathrm{E}-02$ & $-1.11 \mathrm{E}-04$ & 3.75E-01 \\
\hline 100 & 34.0 & 5.93E-05 & 2.01E-09 & $1.01 \mathrm{E}-02$ & $-5.54 \mathrm{E}-04$ & $1.06 \mathrm{E}-02$ & $-1.10 \mathrm{E}-04$ & 3.61E-01 \\
\hline 100 & 33.0 & 5.87E-05 & $9.38 \mathrm{E}-09$ & $9.99 \mathrm{E}-03$ & $-5.54 \mathrm{E}-04$ & $1.05 \mathrm{E}-02$ & $-1.09 \mathrm{E}-04$ & $3.48 \mathrm{E}-01$ \\
\hline 100 & 32.0 & $5.82 \mathrm{E}-05$ & $2.64 \mathrm{E}-08$ & $9.89 \mathrm{E}-03$ & $-5.54 \mathrm{E}-04$ & $1.04 \mathrm{E}-02$ & $-1.08 \mathrm{E}-04$ & 3.34E-01 \\
\hline 100 & 31.0 & $5.76 \mathrm{E}-05$ & $1.57 \mathrm{E}-08$ & $9.78 \mathrm{E}-03$ & $-5.54 \mathrm{E}-04$ & $1.03 \mathrm{E}-02$ & $-1.07 \mathrm{E}-04$ & $3.20 \mathrm{E}-01$ \\
\hline 100 & 30.0 & $5.68 \mathrm{E}-05$ & $8.77 \mathrm{E}-09$ & $9.66 \mathrm{E}-03$ & $-5.54 \mathrm{E}-04$ & $1.02 \mathrm{E}-02$ & $-1.05 \mathrm{E}-04$ & 3.06E-01 \\
\hline 100 & 29.0 & $5.61 \mathrm{E}-05$ & $3.66 \mathrm{E}-09$ & $9.53 \mathrm{E}-03$ & $-5.54 \mathrm{E}-04$ & $1.01 \mathrm{E}-02$ & $-1.04 \mathrm{E}-04$ & 2.92E-01 \\
\hline 100 & 28.0 & $5.53 \mathrm{E}-05$ & 8.60E-09 & $9.40 \mathrm{E}-03$ & $-5.54 \mathrm{E}-04$ & $9.95 \mathrm{E}-03$ & $-1.02 \mathrm{E}-04$ & $2.79 \mathrm{E}-01$ \\
\hline 100 & 27.0 & $5.44 \mathrm{E}-05$ & 6.19E-09 & $9.25 \mathrm{E}-03$ & $-5.54 \mathrm{E}-04$ & $9.80 \mathrm{E}-03$ & $-1.01 \mathrm{E}-04$ & 2.65E-01 \\
\hline 100 & 26.0 & $5.34 \mathrm{E}-05$ & 8.35E-09 & $9.08 \mathrm{E}-03$ & $-5.54 \mathrm{E}-04$ & $9.64 \mathrm{E}-03$ & $-9.90 \mathrm{E}-05$ & $2.51 \mathrm{E}-01$ \\
\hline 100 & 25.0 & $5.24 \mathrm{E}-05$ & 4.18E-09 & 8.91E-03 & $-5.54 \mathrm{E}-04$ & $9.46 \mathrm{E}-03$ & $-9.70 \mathrm{E}-05$ & 2.36E-01 \\
\hline 100 & 24.0 & $5.13 \mathrm{E}-05$ & $1.05 \mathrm{E}-09$ & $8.72 \mathrm{E}-03$ & $-5.54 \mathrm{E}-04$ & $9.27 \mathrm{E}-03$ & $-9.50 \mathrm{E}-05$ & 2.23E-01 \\
\hline 100 & 23.0 & 5.01E-05 & 9.39E-09 & 8.53E-03 & $-5.54 \mathrm{E}-04$ & $9.08 \mathrm{E}-03$ & $-9.29 \mathrm{E}-05$ & $2.09 \mathrm{E}-01$ \\
\hline 100 & 22.0 & 4.89E-05 & 7.87E-09 & 8.32E-03 & $-5.54 \mathrm{E}-04$ & 8.87E-03 & $-9.06 \mathrm{E}-05$ & $1.95 \mathrm{E}-01$ \\
\hline 100 & 21.0 & 4.77E-05 & $1.11 \mathrm{E}-08$ & $8.11 \mathrm{E}-03$ & $-5.54 \mathrm{E}-04$ & 8.66E-03 & $-8.83 \mathrm{E}-05$ & $1.82 \mathrm{E}-01$ \\
\hline 100 & 20.0 & 4.65E-05 & 7.13E-09 & 7.91E-03 & $-5.54 \mathrm{E}-04$ & 8.46E-03 & $-8.61 \mathrm{E}-05$ & $1.69 \mathrm{E}-01$ \\
\hline 100 & 19.0 & 4.53E-05 & $5.16 \mathrm{E}-09$ & 7.71E-03 & $-5.54 \mathrm{E}-04$ & $8.26 \mathrm{E}-03$ & $-8.40 \mathrm{E}-05$ & $1.57 \mathrm{E}-01$ \\
\hline 100 & 18.0 & $4.43 \mathrm{E}-05$ & 6.71E-09 & $7.52 \mathrm{E}-03$ & $-5.54 \mathrm{E}-04$ & $8.08 \mathrm{E}-03$ & $-8.20 \mathrm{E}-05$ & $1.45 \mathrm{E}-01$ \\
\hline
\end{tabular}




$\begin{array}{lllllllll}100 & 17.0 & 4.33 \mathrm{E}-05 & 1.28 \mathrm{E}-08 & 7.37 \mathrm{E}-03 & -5.54 \mathrm{E}-04 & 7.92 \mathrm{E}-03 & -8.02 \mathrm{E}-05 & 1.35 \mathrm{E}-01 \\ 100 & 16.0 & 4.26 \mathrm{E}-05 & 9.60 \mathrm{E}-09 & 7.24 \mathrm{E}-03 & -5.54 \mathrm{E}-04 & 7.80 \mathrm{E}-03 & -7.89 \mathrm{E}-05 & 1.25 \mathrm{E}-01 \\ 100 & 15.0 & 4.22 \mathrm{E}-05 & 2.48 \mathrm{E}-09 & 7.17 \mathrm{E}-03 & -5.54 \mathrm{E}-04 & 7.73 \mathrm{E}-03 & -7.81 \mathrm{E}-05 & 1.16 \mathrm{E}-01 \\ 100 & 14.0 & 4.22 \mathrm{E}-05 & 9.10 \mathrm{E}-10 & 7.17 \mathrm{E}-03 & -5.54 \mathrm{E}-04 & 7.72 \mathrm{E}-03 & -7.81 \mathrm{E}-05 & 1.08 \mathrm{E}-01 \\ 100 & 13.0 & 4.27 \mathrm{E}-05 & 5.14 \mathrm{E}-09 & 7.25 \mathrm{E}-03 & -5.54 \mathrm{E}-04 & 7.81 \mathrm{E}-03 & -7.90 \mathrm{E}-05 & 1.02 \mathrm{E}-01 \\ 100 & 12.0 & 4.38 \mathrm{E}-05 & 4.58 \mathrm{E}-09 & 7.44 \mathrm{E}-03 & -5.54 \mathrm{E}-04 & 7.99 \mathrm{E}-03 & -8.11 \mathrm{E}-05 & 9.59 \mathrm{E}-02 \\ 100 & 11.0 & 4.57 \mathrm{E}-05 & 4.66 \mathrm{E}-09 & 7.76 \mathrm{E}-03 & -5.54 \mathrm{E}-04 & 8.32 \mathrm{E}-03 & -8.46 \mathrm{E}-05 & 9.15 \mathrm{E}-02 \\ 100 & 10.0 & 4.84 \mathrm{E}-05 & 1.38 \mathrm{E}-08 & 8.23 \mathrm{E}-03 & -5.54 \mathrm{E}-04 & 8.79 \mathrm{E}-03 & -8.97 \mathrm{E}-05 & 8.80 \mathrm{E}-02 \\ 100 & 9.0 & 5.22 \mathrm{E}-05 & 2.91 \mathrm{E}-08 & 8.88 \mathrm{E}-03 & -5.54 \mathrm{E}-04 & 9.44 \mathrm{E}-03 & -9.68 \mathrm{E}-05 & 8.50 \mathrm{E}-02 \\ 100 & 8.0 & 5.73 \mathrm{E}-05 & 4.16 \mathrm{E}-08 & 9.75 \mathrm{E}-03 & -5.54 \mathrm{E}-04 & 1.03 \mathrm{E}-02 & -1.06 \mathrm{E}-04 & 8.25 \mathrm{E}-02 \\ 100 & 7.0 & 6.41 \mathrm{E}-05 & 5.30 \mathrm{E}-08 & 1.09 \mathrm{E}-02 & -5.54 \mathrm{E}-04 & 1.14 \mathrm{E}-02 & -1.19 \mathrm{E}-04 & 8.02 \mathrm{E}-02 \\ 100 & 6.0 & 7.3 \mathrm{E}-05 & 9.72 \mathrm{E}-08 & 1.24 \mathrm{E}-02 & -5.54 \mathrm{E}-04 & 1.30 \mathrm{E}-02 & -1.35 \mathrm{E}-04 & 7.79 \mathrm{E}-02 \\ 100 & 5.0 & 8.53 \mathrm{E}-05 & 9.54 \mathrm{E}-08 & 1.45 \mathrm{E}-02 & -5.54 \mathrm{E}-04 & 1.50 \mathrm{E}-02 & -1.58 \mathrm{E}-04 & 7.53 \mathrm{E}-02\end{array}$


Complex 3 ([Mn $\left.\left.\left(\mathrm{CH}_{2} \mathrm{SiMe}_{3}\right)_{2}(\mathrm{dmpe})\right]\right)$

Mass (mg): 3.2

\begin{tabular}{|c|c|c|c|c|c|c|c|c|}
\hline $\begin{array}{l}\text { Field } \\
\text { (Oe) }\end{array}$ & Temp (K) & EMU & STD & $\begin{array}{c}\text { Chi } \\
\text { (cm3/mol) }\end{array}$ & $\begin{array}{c}\mathrm{dia} \\
(\mathrm{cm} 3 / \mathrm{mol})\end{array}$ & $\begin{array}{l}\text { Cor Chi } \\
\text { (cm3/mol) }\end{array}$ & esd & $\begin{array}{c}\text { Chi*T } \\
(\mathrm{cm} 3 \mathrm{~K} / \mathrm{mol})\end{array}$ \\
\hline 10000 & 300.0 & $1.14 \mathrm{E}-03$ & $4.96 \mathrm{E}-08$ & $1.37 \mathrm{E}-02$ & $-2.88 \mathrm{E}-04$ & $1.40 \mathrm{E}-02$ & $-1.18 \mathrm{E}-03$ & $4.19 \mathrm{E}+00$ \\
\hline 10000 & 294.7 & $1.16 \mathrm{E}-03$ & $1.27 \mathrm{E}-06$ & $1.40 \mathrm{E}-02$ & $-2.88 \mathrm{E}-04$ & $1.43 \mathrm{E}-02$ & $-1.22 \mathrm{E}-03$ & $4.21 \mathrm{E}+00$ \\
\hline 10000 & 289.6 & $1.18 \mathrm{E}-03$ & $1.52 \mathrm{E}-06$ & $1.43 \mathrm{E}-02$ & $-2.88 \mathrm{E}-04$ & $1.45 \mathrm{E}-02$ & $-1.25 \mathrm{E}-03$ & $4.21 \mathrm{E}+00$ \\
\hline 10000 & 284.6 & $1.21 \mathrm{E}-03$ & $1.43 \mathrm{E}-06$ & $1.45 \mathrm{E}-02$ & $-2.88 \mathrm{E}-04$ & $1.48 \mathrm{E}-02$ & $-1.27 \mathrm{E}-03$ & $4.22 \mathrm{E}+00$ \\
\hline 10000 & 279.6 & $1.23 \mathrm{E}-03$ & $1.28 \mathrm{E}-06$ & $1.48 \mathrm{E}-02$ & $-2.88 \mathrm{E}-04$ & $1.51 \mathrm{E}-02$ & $-1.29 \mathrm{E}-03$ & $4.22 \mathrm{E}+00$ \\
\hline 10000 & 274.6 & $1.25 \mathrm{E}-03$ & $1.34 \mathrm{E}-06$ & $1.51 \mathrm{E}-02$ & $-2.88 \mathrm{E}-04$ & $1.54 \mathrm{E}-02$ & $-1.32 \mathrm{E}-03$ & $4.23 \mathrm{E}+00$ \\
\hline 10000 & 269.6 & $1.28 \mathrm{E}-03$ & $1.30 \mathrm{E}-06$ & $1.54 \mathrm{E}-02$ & $-2.88 \mathrm{E}-04$ & $1.57 \mathrm{E}-02$ & $-1.34 \mathrm{E}-03$ & $4.23 \mathrm{E}+00$ \\
\hline 10000 & 264.6 & $1.30 \mathrm{E}-03$ & $1.29 \mathrm{E}-06$ & $1.57 \mathrm{E}-02$ & $-2.88 \mathrm{E}-04$ & $1.60 \mathrm{E}-02$ & $-1.37 \mathrm{E}-03$ & $4.23 \mathrm{E}+00$ \\
\hline 10000 & 259.6 & $1.33 \mathrm{E}-03$ & $1.34 \mathrm{E}-06$ & $1.60 \mathrm{E}-02$ & $-2.88 \mathrm{E}-04$ & $1.63 \mathrm{E}-02$ & $-1.40 \mathrm{E}-03$ & $4.23 \mathrm{E}+00$ \\
\hline 10000 & 254.6 & $1.36 \mathrm{E}-03$ & $1.59 \mathrm{E}-06$ & $1.63 \mathrm{E}-02$ & $-2.88 \mathrm{E}-04$ & $1.66 \mathrm{E}-02$ & $-1.43 \mathrm{E}-03$ & $4.23 \mathrm{E}+00$ \\
\hline 10000 & 249.6 & $1.38 \mathrm{E}-03$ & $1.36 \mathrm{E}-06$ & $1.67 \mathrm{E}-02$ & $-2.88 \mathrm{E}-04$ & $1.70 \mathrm{E}-02$ & $-1.45 \mathrm{E}-03$ & $4.23 \mathrm{E}+00$ \\
\hline 10000 & 244.7 & $1.41 \mathrm{E}-03$ & $1.60 \mathrm{E}-06$ & $1.70 \mathrm{E}-02$ & $-2.88 \mathrm{E}-04$ & $1.73 \mathrm{E}-02$ & $-1.49 \mathrm{E}-03$ & $4.23 \mathrm{E}+00$ \\
\hline 10000 & 239.7 & $1.44 \mathrm{E}-03$ & $1.62 \mathrm{E}-06$ & $1.74 \mathrm{E}-02$ & $-2.88 \mathrm{E}-04$ & $1.77 \mathrm{E}-02$ & $-1.52 \mathrm{E}-03$ & $4.23 \mathrm{E}+00$ \\
\hline 10000 & 234.7 & $1.47 \mathrm{E}-03$ & $1.66 \mathrm{E}-06$ & $1.77 \mathrm{E}-02$ & $-2.88 \mathrm{E}-04$ & $1.80 \mathrm{E}-02$ & $-1.55 \mathrm{E}-03$ & $4.23 \mathrm{E}+00$ \\
\hline 10000 & 229.7 & $1.51 \mathrm{E}-03$ & $1.63 \mathrm{E}-06$ & $1.81 \mathrm{E}-02$ & $-2.88 \mathrm{E}-04$ & $1.84 \mathrm{E}-02$ & $-1.59 \mathrm{E}-03$ & $4.23 \mathrm{E}+00$ \\
\hline 10000 & 224.7 & $1.54 \mathrm{E}-03$ & $1.67 \mathrm{E}-06$ & $1.86 \mathrm{E}-02$ & $-2.88 \mathrm{E}-04$ & $1.88 \mathrm{E}-02$ & $-1.62 \mathrm{E}-03$ & $4.23 \mathrm{E}+00$ \\
\hline 10000 & 219.7 & $1.58 \mathrm{E}-03$ & $1.87 \mathrm{E}-06$ & $1.90 \mathrm{E}-02$ & $-2.88 \mathrm{E}-04$ & $1.93 \mathrm{E}-02$ & $-1.66 \mathrm{E}-03$ & $4.23 \mathrm{E}+00$ \\
\hline 10000 & 214.7 & $1.61 \mathrm{E}-03$ & $1.92 \mathrm{E}-06$ & $1.94 \mathrm{E}-02$ & $-2.88 \mathrm{E}-04$ & $1.97 \mathrm{E}-02$ & $-1.70 \mathrm{E}-03$ & $4.23 \mathrm{E}+00$ \\
\hline 10000 & 209.7 & $1.65 \mathrm{E}-03$ & $1.85 \mathrm{E}-06$ & 1.99E-02 & $-2.88 \mathrm{E}-04$ & $2.02 \mathrm{E}-02$ & $-1.74 \mathrm{E}-03$ & $4.23 \mathrm{E}+00$ \\
\hline 10000 & 204.7 & $1.69 \mathrm{E}-03$ & $1.92 \mathrm{E}-06$ & $2.04 \mathrm{E}-02$ & $-2.88 \mathrm{E}-04$ & 2.07E-02 & $-1.78 \mathrm{E}-03$ & $4.23 \mathrm{E}+00$ \\
\hline 10000 & 199.8 & $1.73 \mathrm{E}-03$ & $2.00 \mathrm{E}-06$ & 2.09E-02 & $-2.88 \mathrm{E}-04$ & $2.12 \mathrm{E}-02$ & $-1.83 \mathrm{E}-03$ & $4.23 \mathrm{E}+00$ \\
\hline 10000 & 194.8 & $1.78 \mathrm{E}-03$ & $2.04 \mathrm{E}-06$ & $2.14 \mathrm{E}-02$ & $-2.88 \mathrm{E}-04$ & $2.17 \mathrm{E}-02$ & $-1.87 \mathrm{E}-03$ & $4.23 \mathrm{E}+00$ \\
\hline 10000 & 189.8 & $1.82 \mathrm{E}-03$ & $2.10 \mathrm{E}-06$ & $2.20 \mathrm{E}-02$ & $-2.88 \mathrm{E}-04$ & $2.23 \mathrm{E}-02$ & $-1.92 \mathrm{E}-03$ & $4.23 \mathrm{E}+00$ \\
\hline 10000 & 184.8 & $1.87 \mathrm{E}-03$ & $2.26 \mathrm{E}-06$ & $2.26 \mathrm{E}-02$ & $-2.88 \mathrm{E}-04$ & $2.29 \mathrm{E}-02$ & $-1.98 \mathrm{E}-03$ & $4.22 \mathrm{E}+00$ \\
\hline 10000 & 179.8 & $1.93 \mathrm{E}-03$ & $2.86 \mathrm{E}-06$ & 2.32E-02 & $-2.88 \mathrm{E}-04$ & $2.35 \mathrm{E}-02$ & $-2.04 \mathrm{E}-03$ & $4.22 \mathrm{E}+00$ \\
\hline 10000 & 174.8 & $1.98 \mathrm{E}-03$ & $2.37 \mathrm{E}-06$ & $2.39 \mathrm{E}-02$ & $-2.88 \mathrm{E}-04$ & $2.42 \mathrm{E}-02$ & $-2.09 \mathrm{E}-03$ & $4.22 \mathrm{E}+00$ \\
\hline 10000 & 169.8 & $2.04 \mathrm{E}-03$ & $2.52 \mathrm{E}-06$ & $2.46 \mathrm{E}-02$ & $-2.88 \mathrm{E}-04$ & $2.49 \mathrm{E}-02$ & $-2.15 E-03$ & $4.22 \mathrm{E}+00$ \\
\hline 10000 & 164.8 & $2.10 \mathrm{E}-03$ & $2.60 \mathrm{E}-06$ & $2.53 \mathrm{E}-02$ & $-2.88 \mathrm{E}-04$ & $2.56 \mathrm{E}-02$ & $-2.22 \mathrm{E}-03$ & $4.22 \mathrm{E}+00$ \\
\hline 10000 & 159.8 & $2.17 \mathrm{E}-03$ & $2.59 \mathrm{E}-06$ & $2.62 \mathrm{E}-02$ & $-2.88 \mathrm{E}-04$ & 2.64E-02 & $-2.29 \mathrm{E}-03$ & $4.23 \mathrm{E}+00$ \\
\hline 10000 & 154.9 & $2.24 \mathrm{E}-03$ & $2.78 \mathrm{E}-06$ & $2.70 \mathrm{E}-02$ & $-2.88 \mathrm{E}-04$ & $2.73 \mathrm{E}-02$ & $-2.36 \mathrm{E}-03$ & $4.23 \mathrm{E}+00$ \\
\hline 10000 & 149.9 & $2.32 \mathrm{E}-03$ & $2.85 \mathrm{E}-06$ & 2.79E-02 & $-2.88 \mathrm{E}-04$ & $2.82 \mathrm{E}-02$ & $-2.44 \mathrm{E}-03$ & $4.23 \mathrm{E}+00$ \\
\hline 10000 & 144.9 & $2.40 \mathrm{E}-03$ & $2.94 \mathrm{E}-06$ & $2.89 \mathrm{E}-02$ & $-2.88 \mathrm{E}-04$ & $2.92 \mathrm{E}-02$ & $-2.53 \mathrm{E}-03$ & $4.22 \mathrm{E}+00$ \\
\hline 10000 & 139.9 & $2.48 \mathrm{E}-03$ & $3.12 \mathrm{E}-06$ & $2.99 \mathrm{E}-02$ & $-2.88 \mathrm{E}-04$ & $3.02 \mathrm{E}-02$ & $-2.62 \mathrm{E}-03$ & $4.22 \mathrm{E}+00$ \\
\hline 10000 & 134.9 & $2.58 \mathrm{E}-03$ & $3.35 \mathrm{E}-06$ & $3.10 \mathrm{E}-02$ & $-2.88 \mathrm{E}-04$ & $3.13 \mathrm{E}-02$ & $-2.72 \mathrm{E}-03$ & $4.22 \mathrm{E}+00$ \\
\hline 10000 & 129.9 & $2.67 \mathrm{E}-03$ & $3.40 \mathrm{E}-06$ & $3.22 \mathrm{E}-02$ & $-2.88 \mathrm{E}-04$ & $3.25 \mathrm{E}-02$ & $-2.82 \mathrm{E}-03$ & $4.22 \mathrm{E}+00$ \\
\hline 10000 & 124.9 & $2.78 \mathrm{E}-03$ & $3.56 \mathrm{E}-06$ & $3.35 \mathrm{E}-02$ & $-2.88 \mathrm{E}-04$ & $3.38 \mathrm{E}-02$ & $-2.94 \mathrm{E}-03$ & $4.22 \mathrm{E}+00$ \\
\hline 10000 & 119.9 & $2.90 \mathrm{E}-03$ & $3.73 \mathrm{E}-06$ & $3.49 \mathrm{E}-02$ & $-2.88 \mathrm{E}-04$ & $3.52 \mathrm{E}-02$ & $-3.06 \mathrm{E}-03$ & $4.22 \mathrm{E}+00$ \\
\hline 10000 & 115.0 & $3.02 \mathrm{E}-03$ & $3.79 \mathrm{E}-06$ & $3.64 \mathrm{E}-02$ & $-2.88 \mathrm{E}-04$ & $3.67 \mathrm{E}-02$ & $-3.19 \mathrm{E}-03$ & $4.22 \mathrm{E}+00$ \\
\hline 10000 & 110.0 & $3.16 \mathrm{E}-03$ & $4.24 \mathrm{E}-06$ & $3.81 \mathrm{E}-02$ & $-2.88 \mathrm{E}-04$ & $3.84 \mathrm{E}-02$ & $-3.34 \mathrm{E}-03$ & $4.22 \mathrm{E}+00$ \\
\hline
\end{tabular}




\begin{tabular}{|c|c|c|c|c|c|c|c|c|}
\hline 10000 & 105.0 & $3.31 \mathrm{E}-03$ & $4.15 \mathrm{E}-06$ & $3.99 \mathrm{E}-02$ & $-2.88 \mathrm{E}-04$ & $4.02 \mathrm{E}-02$ & $-3.49 \mathrm{E}-03$ & $4.22 \mathrm{E}+00$ \\
\hline 10000 & 100.0 & $3.48 \mathrm{E}-03$ & $4.56 \mathrm{E}-06$ & 4.19E-02 & $-2.88 \mathrm{E}-04$ & 4.22E-02 & $-3.67 \mathrm{E}-03$ & $4.22 \mathrm{E}+00$ \\
\hline 10000 & 98.9 & $3.50 \mathrm{E}-03$ & $1.94 \mathrm{E}-06$ & 4.22E-02 & $-2.88 \mathrm{E}-04$ & $4.25 \mathrm{E}-02$ & $-3.66 \mathrm{E}-03$ & $4.21 \mathrm{E}+00$ \\
\hline 10000 & 97.9 & $3.55 \mathrm{E}-03$ & $3.96 \mathrm{E}-06$ & 4.27E-02 & $-2.88 \mathrm{E}-04$ & $4.30 \mathrm{E}-02$ & $-3.73 E-03$ & $4.21 \mathrm{E}+00$ \\
\hline 10000 & 96.9 & $3.58 \mathrm{E}-03$ & $3.66 \mathrm{E}-06$ & $4.31 \mathrm{E}-02$ & $-2.88 \mathrm{E}-04$ & 4.34E-02 & $-3.77 \mathrm{E}-03$ & $4.21 \mathrm{E}+00$ \\
\hline 10000 & 95.9 & $3.62 \mathrm{E}-03$ & $3.96 \mathrm{E}-06$ & $4.36 \mathrm{E}-02$ & $-2.88 \mathrm{E}-04$ & 4.39E-02 & $-3.81 \mathrm{E}-03$ & $4.21 \mathrm{E}+00$ \\
\hline 10000 & 94.9 & $3.66 \mathrm{E}-03$ & 4.13E-06 & $4.40 \mathrm{E}-02$ & $-2.88 \mathrm{E}-04$ & 4.43E-02 & $-3.85 E-03$ & $4.21 \mathrm{E}+00$ \\
\hline 10000 & 93.9 & 3.69E-03 & 4.19E-06 & 4.45E-02 & $-2.88 \mathrm{E}-04$ & 4.48E-02 & $-3.89 \mathrm{E}-03$ & $4.21 \mathrm{E}+00$ \\
\hline 10000 & 92.9 & $3.73 \mathrm{E}-03$ & 4.24E-06 & $4.50 \mathrm{E}-02$ & $-2.88 \mathrm{E}-04$ & 4.53E-02 & $-3.93 \mathrm{E}-03$ & $4.21 \mathrm{E}+00$ \\
\hline 10000 & 91.9 & $3.78 \mathrm{E}-03$ & $4.48 \mathrm{E}-06$ & $4.55 \mathrm{E}-02$ & $-2.88 \mathrm{E}-04$ & $4.58 \mathrm{E}-02$ & $-3.98 \mathrm{E}-03$ & $4.21 \mathrm{E}+00$ \\
\hline 10000 & 90.9 & $3.82 \mathrm{E}-03$ & 4.59E-06 & 4.60E-02 & $-2.88 \mathrm{E}-04$ & 4.63E-02 & $-4.02 \mathrm{E}-03$ & $4.21 \mathrm{E}+00$ \\
\hline 10000 & 89.9 & $3.86 \mathrm{E}-03$ & 5.13E-06 & $4.65 \mathrm{E}-02$ & $-2.88 \mathrm{E}-04$ & $4.68 \mathrm{E}-02$ & $-4.08 \mathrm{E}-03$ & $4.21 \mathrm{E}+00$ \\
\hline 10000 & 88.9 & $3.90 \mathrm{E}-03$ & 4.91E-06 & 4.70E-02 & $-2.88 \mathrm{E}-04$ & 4.73E-02 & $-4.12 \mathrm{E}-03$ & $4.21 \mathrm{E}+00$ \\
\hline 10000 & 87.9 & $3.95 \mathrm{E}-03$ & 5.13E-06 & $4.75 \mathrm{E}-02$ & $-2.88 \mathrm{E}-04$ & $4.78 \mathrm{E}-02$ & $-4.17 \mathrm{E}-03$ & $4.20 \mathrm{E}+00$ \\
\hline 10000 & 86.9 & $3.99 \mathrm{E}-03$ & $5.29 \mathrm{E}-06$ & $4.81 \mathrm{E}-02$ & $-2.88 \mathrm{E}-04$ & 4.84E-02 & $-4.21 \mathrm{E}-03$ & $4.20 \mathrm{E}+00$ \\
\hline 10000 & 85.9 & 4.04E-03 & $5.65 \mathrm{E}-06$ & $4.86 \mathrm{E}-02$ & $-2.88 \mathrm{E}-04$ & 4.89E-02 & $-4.27 \mathrm{E}-03$ & $4.20 \mathrm{E}+00$ \\
\hline 10000 & 84.9 & $4.08 \mathrm{E}-03$ & 5.92E-06 & 4.92E-02 & $-2.88 \mathrm{E}-04$ & 4.95E-02 & $-4.32 \mathrm{E}-03$ & $4.20 \mathrm{E}+00$ \\
\hline 10000 & 83.9 & $4.13 \mathrm{E}-03$ & $5.89 \mathrm{E}-06$ & $4.98 \mathrm{E}-02$ & $-2.88 \mathrm{E}-04$ & $5.01 \mathrm{E}-02$ & $-4.37 \mathrm{E}-03$ & $4.20 \mathrm{E}+00$ \\
\hline 10000 & 82.9 & 4.18E-03 & $6.13 \mathrm{E}-06$ & $5.04 \mathrm{E}-02$ & $-2.88 \mathrm{E}-04$ & 5.07E-02 & $-4.42 \mathrm{E}-03$ & $4.20 \mathrm{E}+00$ \\
\hline 10000 & 81.9 & 4.23E-03 & $6.33 \mathrm{E}-06$ & $5.10 \mathrm{E}-02$ & $-2.88 \mathrm{E}-04$ & $5.13 \mathrm{E}-02$ & $-4.48 \mathrm{E}-03$ & $4.20 \mathrm{E}+00$ \\
\hline 10000 & 80.9 & 4.29E-03 & $6.61 \mathrm{E}-06$ & $5.16 \mathrm{E}-02$ & $-2.88 \mathrm{E}-04$ & $5.19 \mathrm{E}-02$ & $-4.54 \mathrm{E}-03$ & $4.20 \mathrm{E}+00$ \\
\hline 10000 & 79.9 & 4.34E-03 & $6.58 \mathrm{E}-06$ & $5.23 \mathrm{E}-02$ & $-2.88 \mathrm{E}-04$ & $5.26 \mathrm{E}-02$ & $-4.59 \mathrm{E}-03$ & $4.20 \mathrm{E}+00$ \\
\hline 10000 & 78.9 & 4.39E-03 & $6.71 \mathrm{E}-06$ & $5.29 \mathrm{E}-02$ & $-2.88 \mathrm{E}-04$ & $5.32 \mathrm{E}-02$ & $-4.65 \mathrm{E}-03$ & $4.20 \mathrm{E}+00$ \\
\hline 10000 & 77.9 & $4.45 \mathrm{E}-03$ & $7.16 \mathrm{E}-06$ & $5.36 \mathrm{E}-02$ & $-2.88 \mathrm{E}-04$ & 5.39E-02 & $-4.72 \mathrm{E}-03$ & $4.20 \mathrm{E}+00$ \\
\hline 10000 & 77.0 & 4.51E-03 & 7.14E-06 & $5.43 \mathrm{E}-02$ & $-2.88 \mathrm{E}-04$ & $5.46 \mathrm{E}-02$ & $-4.77 \mathrm{E}-03$ & $4.20 \mathrm{E}+00$ \\
\hline 10000 & 76.0 & 4.57E-03 & 7.31E-06 & $5.50 \mathrm{E}-02$ & $-2.88 \mathrm{E}-04$ & $5.53 \mathrm{E}-02$ & $-4.84 \mathrm{E}-03$ & $4.20 \mathrm{E}+00$ \\
\hline 10000 & 75.0 & 4.63E-03 & 7.47E-06 & $5.57 \mathrm{E}-02$ & $-2.88 \mathrm{E}-04$ & $5.60 \mathrm{E}-02$ & $-4.90 \mathrm{E}-03$ & $4.20 \mathrm{E}+00$ \\
\hline 10000 & 74.0 & 4.69E-03 & 7.30E-06 & $5.65 \mathrm{E}-02$ & $-2.88 \mathrm{E}-04$ & $5.68 \mathrm{E}-02$ & $-4.96 \mathrm{E}-03$ & $4.20 \mathrm{E}+00$ \\
\hline 10000 & 73.0 & 4.75E-03 & 7.29E-06 & $5.72 \mathrm{E}-02$ & $-2.88 \mathrm{E}-04$ & $5.75 \mathrm{E}-02$ & $-5.03 \mathrm{E}-03$ & $4.20 \mathrm{E}+00$ \\
\hline 10000 & 72.0 & 4.82E-03 & $7.51 \mathrm{E}-06$ & $5.80 \mathrm{E}-02$ & $-2.88 \mathrm{E}-04$ & $5.83 \mathrm{E}-02$ & $-5.10 \mathrm{E}-03$ & $4.20 \mathrm{E}+00$ \\
\hline 10000 & 71.0 & 4.88E-03 & 7.38E-06 & $5.88 \mathrm{E}-02$ & $-2.88 \mathrm{E}-04$ & $5.91 \mathrm{E}-02$ & $-5.17 \mathrm{E}-03$ & $4.20 \mathrm{E}+00$ \\
\hline 10000 & 70.0 & 4.95E-03 & 7.27E-06 & $5.97 \mathrm{E}-02$ & $-2.88 \mathrm{E}-04$ & $5.99 \mathrm{E}-02$ & $-5.24 \mathrm{E}-03$ & $4.20 \mathrm{E}+00$ \\
\hline 10000 & 69.0 & $5.02 \mathrm{E}-03$ & 7.91E-06 & $6.05 \mathrm{E}-02$ & $-2.88 \mathrm{E}-04$ & $6.08 \mathrm{E}-02$ & $-5.32 \mathrm{E}-03$ & $4.19 \mathrm{E}+00$ \\
\hline 10000 & 68.0 & $5.09 \mathrm{E}-03$ & 7.75E-06 & $6.14 \mathrm{E}-02$ & $-2.88 \mathrm{E}-04$ & $6.17 \mathrm{E}-02$ & $-5.39 \mathrm{E}-03$ & $4.19 \mathrm{E}+00$ \\
\hline 10000 & 67.0 & $5.17 \mathrm{E}-03$ & 7.49E-06 & $6.23 \mathrm{E}-02$ & $-2.88 \mathrm{E}-04$ & $6.25 \mathrm{E}-02$ & $-5.46 \mathrm{E}-03$ & $4.19 \mathrm{E}+00$ \\
\hline 10000 & 66.0 & $5.24 \mathrm{E}-03$ & $6.42 \mathrm{E}-06$ & 6.32E-02 & $-2.88 \mathrm{E}-04$ & $6.35 \mathrm{E}-02$ & $-5.53 \mathrm{E}-03$ & $4.19 \mathrm{E}+00$ \\
\hline 10000 & 65.0 & $5.32 \mathrm{E}-03$ & 7.22E-06 & $6.41 \mathrm{E}-02$ & $-2.88 \mathrm{E}-04$ & $6.44 \mathrm{E}-02$ & $-5.62 \mathrm{E}-03$ & $4.19 \mathrm{E}+00$ \\
\hline 10000 & 64.0 & $5.41 \mathrm{E}-03$ & $6.85 \mathrm{E}-06$ & $6.51 \mathrm{E}-02$ & $-2.88 \mathrm{E}-04$ & $6.54 \mathrm{E}-02$ & $-5.70 \mathrm{E}-03$ & $4.19 \mathrm{E}+00$ \\
\hline 10000 & 63.0 & $5.49 \mathrm{E}-03$ & $7.26 \mathrm{E}-06$ & $6.62 \mathrm{E}-02$ & $-2.88 \mathrm{E}-04$ & $6.64 \mathrm{E}-02$ & $-5.80 \mathrm{E}-03$ & $4.19 \mathrm{E}+00$ \\
\hline 10000 & 62.0 & $5.58 \mathrm{E}-03$ & $6.82 \mathrm{E}-06$ & 6.72E-02 & $-2.88 \mathrm{E}-04$ & $6.75 \mathrm{E}-02$ & $-5.88 \mathrm{E}-03$ & $4.19 \mathrm{E}+00$ \\
\hline 10000 & 61.0 & $5.67 \mathrm{E}-03$ & $6.60 \mathrm{E}-06$ & $6.83 \mathrm{E}-02$ & $-2.88 \mathrm{E}-04$ & $6.86 \mathrm{E}-02$ & $-5.97 \mathrm{E}-03$ & $4.19 \mathrm{E}+00$ \\
\hline 10000 & 60.0 & $5.76 \mathrm{E}-03$ & $1.97 \mathrm{E}-07$ & $6.93 \mathrm{E}-02$ & $-2.88 \mathrm{E}-04$ & $6.96 \mathrm{E}-02$ & $-5.98 \mathrm{E}-03$ & $4.18 \mathrm{E}+00$ \\
\hline 10000 & 59.0 & $5.85 \mathrm{E}-03$ & $1.84 \mathrm{E}-06$ & 7.05E-02 & $-2.88 \mathrm{E}-04$ & $7.08 \mathrm{E}-02$ & $-6.10 \mathrm{E}-03$ & $4.18 \mathrm{E}+00$ \\
\hline
\end{tabular}




\begin{tabular}{|c|c|c|c|c|c|c|c|c|}
\hline 10000 & 58.0 & $5.95 \mathrm{E}-03$ & $9.09 \mathrm{E}-08$ & 7.17E-02 & $-2.88 \mathrm{E}-04$ & $7.20 \mathrm{E}-02$ & $-6.18 \mathrm{E}-03$ & $4.18 \mathrm{E}+00$ \\
\hline 10000 & 57.0 & $6.06 \mathrm{E}-03$ & $2.30 \mathrm{E}-07$ & $7.30 \mathrm{E}-02$ & $-2.88 \mathrm{E}-04$ & 7.33E-02 & $-6.29 \mathrm{E}-03$ & $4.18 \mathrm{E}+00$ \\
\hline 10000 & 56.0 & $6.16 \mathrm{E}-03$ & $2.14 \mathrm{E}-07$ & 7.43E-02 & $-2.88 \mathrm{E}-04$ & $7.46 \mathrm{E}-02$ & $-6.40 \mathrm{E}-03$ & $4.18 \mathrm{E}+00$ \\
\hline 10000 & 55.0 & $6.28 \mathrm{E}-03$ & $2.36 \mathrm{E}-07$ & $7.56 \mathrm{E}-02$ & $-2.88 \mathrm{E}-04$ & 7.59E-02 & $-6.52 E-03$ & $4.18 \mathrm{E}+00$ \\
\hline 10000 & 54.0 & $6.39 \mathrm{E}-03$ & $5.58 \mathrm{E}-07$ & 7.70E-02 & $-2.88 \mathrm{E}-04$ & 7.73E-02 & $-6.65 \mathrm{E}-03$ & $4.18 \mathrm{E}+00$ \\
\hline 10000 & 53.0 & $6.51 \mathrm{E}-03$ & $3.82 \mathrm{E}-07$ & $7.85 \mathrm{E}-02$ & $-2.88 \mathrm{E}-04$ & 7.87E-02 & $-6.77 \mathrm{E}-03$ & $4.17 \mathrm{E}+00$ \\
\hline 10000 & 52.0 & 6.64E-03 & $2.84 \mathrm{E}-07$ & $8.00 \mathrm{E}-02$ & $-2.88 \mathrm{E}-04$ & 8.02E-02 & $-6.90 \mathrm{E}-03$ & $4.17 \mathrm{E}+00$ \\
\hline 10000 & 51.0 & $6.77 \mathrm{E}-03$ & $4.74 \mathrm{E}-07$ & $8.15 \mathrm{E}-02$ & $-2.88 \mathrm{E}-04$ & $8.18 \mathrm{E}-02$ & $-7.03 \mathrm{E}-03$ & $4.17 \mathrm{E}+00$ \\
\hline 10000 & 50.0 & $6.90 \mathrm{E}-03$ & $7.60 \mathrm{E}-07$ & $8.31 \mathrm{E}-02$ & $-2.88 \mathrm{E}-04$ & 8.34E-02 & $-7.18 \mathrm{E}-03$ & $4.17 \mathrm{E}+00$ \\
\hline 10000 & 49.0 & 7.05E-03 & $1.03 \mathrm{E}-06$ & $8.49 \mathrm{E}-02$ & $-2.88 \mathrm{E}-04$ & 8.52E-02 & $-7.33 \mathrm{E}-03$ & $4.18 \mathrm{E}+00$ \\
\hline 10000 & 48.0 & 7.19E-03 & $6.38 \mathrm{E}-07$ & $8.66 \mathrm{E}-02$ & $-2.88 \mathrm{E}-04$ & 8.69E-02 & $-7.48 \mathrm{E}-03$ & $4.18 \mathrm{E}+00$ \\
\hline 10000 & 47.0 & 7.34E-03 & 5.97E-07 & $8.85 \mathrm{E}-02$ & $-2.88 \mathrm{E}-04$ & $8.88 \mathrm{E}-02$ & $-7.64 \mathrm{E}-03$ & $4.18 \mathrm{E}+00$ \\
\hline 10000 & 46.0 & 7.50E-03 & $1.51 \mathrm{E}-07$ & $9.04 \mathrm{E}-02$ & $-2.88 \mathrm{E}-04$ & $9.07 \mathrm{E}-02$ & $-7.80 \mathrm{E}-03$ & $4.17 \mathrm{E}+00$ \\
\hline 10000 & 45.0 & 7.67E-03 & 4.12E-07 & $9.24 \mathrm{E}-02$ & $-2.88 \mathrm{E}-04$ & $9.27 \mathrm{E}-02$ & $-7.97 \mathrm{E}-03$ & $4.17 \mathrm{E}+00$ \\
\hline 10000 & 44.0 & 7.84E-03 & $1.40 \mathrm{E}-06$ & $9.45 \mathrm{E}-02$ & $-2.88 \mathrm{E}-04$ & $9.48 \mathrm{E}-02$ & $-8.16 \mathrm{E}-03$ & $4.17 \mathrm{E}+00$ \\
\hline 10000 & 43.0 & 8.02E-03 & $5.68 \mathrm{E}-07$ & $9.67 \mathrm{E}-02$ & $-2.88 \mathrm{E}-04$ & $9.70 \mathrm{E}-02$ & $-8.34 \mathrm{E}-03$ & $4.17 \mathrm{E}+00$ \\
\hline 10000 & 42.0 & $8.21 \mathrm{E}-03$ & $6.76 \mathrm{E}-07$ & $9.89 \mathrm{E}-02$ & $-2.88 \mathrm{E}-04$ & $9.92 \mathrm{E}-02$ & $-8.54 \mathrm{E}-03$ & $4.17 \mathrm{E}+00$ \\
\hline 10000 & 41.0 & $8.41 \mathrm{E}-03$ & $1.86 \mathrm{E}-06$ & $1.01 \mathrm{E}-01$ & $-2.88 \mathrm{E}-04$ & $1.02 \mathrm{E}-01$ & $-8.76 \mathrm{E}-03$ & $4.17 \mathrm{E}+00$ \\
\hline 10000 & 40.0 & 8.62E-03 & $2.60 \mathrm{E}-07$ & $1.04 \mathrm{E}-01$ & $-2.88 \mathrm{E}-04$ & $1.04 \mathrm{E}-01$ & $-8.96 \mathrm{E}-03$ & $4.17 \mathrm{E}+00$ \\
\hline 10000 & 39.0 & $8.84 \mathrm{E}-03$ & 4.39E-07 & $1.07 \mathrm{E}-01$ & $-2.88 \mathrm{E}-04$ & 1.07E-01 & $-9.19 \mathrm{E}-03$ & $4.17 \mathrm{E}+00$ \\
\hline 10000 & 38.0 & $9.07 \mathrm{E}-03$ & $5.02 \mathrm{E}-07$ & $1.09 \mathrm{E}-01$ & $-2.88 \mathrm{E}-04$ & $1.10 \mathrm{E}-01$ & $-9.43 \mathrm{E}-03$ & $4.16 \mathrm{E}+00$ \\
\hline 10000 & 37.0 & $9.31 \mathrm{E}-03$ & $1.90 \mathrm{E}-07$ & $1.12 \mathrm{E}-01$ & $-2.88 \mathrm{E}-04$ & $1.13 \mathrm{E}-01$ & $-9.68 \mathrm{E}-03$ & $4.16 \mathrm{E}+00$ \\
\hline 10000 & 36.0 & $9.57 \mathrm{E}-03$ & $3.19 \mathrm{E}-07$ & $1.15 \mathrm{E}-01$ & $-2.88 \mathrm{E}-04$ & $1.16 \mathrm{E}-01$ & $-9.94 \mathrm{E}-03$ & $4.16 \mathrm{E}+00$ \\
\hline 10000 & 35.0 & $9.84 \mathrm{E}-03$ & $5.41 \mathrm{E}-07$ & $1.19 \mathrm{E}-01$ & $-2.88 \mathrm{E}-04$ & $1.19 \mathrm{E}-01$ & $-1.02 \mathrm{E}-02$ & $4.16 \mathrm{E}+00$ \\
\hline 10000 & 34.0 & $1.01 \mathrm{E}-02$ & $1.69 \mathrm{E}-06$ & $1.22 \mathrm{E}-01$ & $-2.88 \mathrm{E}-04$ & $1.22 \mathrm{E}-01$ & $-1.05 \mathrm{E}-02$ & $4.16 \mathrm{E}+00$ \\
\hline 10000 & 33.0 & $1.04 \mathrm{E}-02$ & $7.31 \mathrm{E}-07$ & $1.26 \mathrm{E}-01$ & $-2.88 \mathrm{E}-04$ & $1.26 \mathrm{E}-01$ & $-1.08 \mathrm{E}-02$ & $4.16 \mathrm{E}+00$ \\
\hline 10000 & 32.0 & $1.08 \mathrm{E}-02$ & $9.25 \mathrm{E}-07$ & $1.30 \mathrm{E}-01$ & $-2.88 \mathrm{E}-04$ & $1.30 \mathrm{E}-01$ & $-1.12 \mathrm{E}-02$ & $4.16 \mathrm{E}+00$ \\
\hline 10000 & 31.0 & $1.11 \mathrm{E}-02$ & 7.62E-07 & $1.34 \mathrm{E}-01$ & $-2.88 \mathrm{E}-04$ & $1.34 \mathrm{E}-01$ & $-1.15 \mathrm{E}-02$ & $4.15 \mathrm{E}+00$ \\
\hline 10000 & 30.0 & $1.15 \mathrm{E}-02$ & $7.70 \mathrm{E}-07$ & $1.38 \mathrm{E}-01$ & $-2.88 \mathrm{E}-04$ & $1.38 \mathrm{E}-01$ & $-1.19 \mathrm{E}-02$ & $4.15 \mathrm{E}+00$ \\
\hline 10000 & 29.0 & 1.19E-02 & $7.50 \mathrm{E}-07$ & $1.43 \mathrm{E}-01$ & $-2.88 \mathrm{E}-04$ & $1.43 \mathrm{E}-01$ & $-1.23 \mathrm{E}-02$ & $4.15 \mathrm{E}+00$ \\
\hline 10000 & 28.0 & $1.23 \mathrm{E}-02$ & $1.66 \mathrm{E}-06$ & $1.48 \mathrm{E}-01$ & $-2.88 \mathrm{E}-04$ & $1.48 \mathrm{E}-01$ & $-1.28 \mathrm{E}-02$ & $4.15 \mathrm{E}+00$ \\
\hline 10000 & 27.0 & $1.27 \mathrm{E}-02$ & $1.44 \mathrm{E}-06$ & $1.53 \mathrm{E}-01$ & $-2.88 \mathrm{E}-04$ & $1.54 \mathrm{E}-01$ & $-1.32 \mathrm{E}-02$ & $4.14 \mathrm{E}+00$ \\
\hline 10000 & 26.0 & $1.32 \mathrm{E}-02$ & $1.47 \mathrm{E}-06$ & $1.59 \mathrm{E}-01$ & $-2.88 \mathrm{E}-04$ & $1.59 \mathrm{E}-01$ & $-1.37 \mathrm{E}-02$ & $4.14 \mathrm{E}+00$ \\
\hline 10000 & 25.0 & $1.37 \mathrm{E}-02$ & $1.07 \mathrm{E}-05$ & $1.65 \mathrm{E}-01$ & $-2.88 \mathrm{E}-04$ & $1.65 \mathrm{E}-01$ & $-1.44 \mathrm{E}-02$ & $4.14 \mathrm{E}+00$ \\
\hline 10000 & 24.0 & $1.43 \mathrm{E}-02$ & $3.64 \mathrm{E}-06$ & $1.72 \mathrm{E}-01$ & $-2.88 \mathrm{E}-04$ & $1.72 \mathrm{E}-01$ & $-1.49 \mathrm{E}-02$ & $4.14 \mathrm{E}+00$ \\
\hline 10000 & 23.0 & $1.49 \mathrm{E}-02$ & $3.68 \mathrm{E}-06$ & $1.79 \mathrm{E}-01$ & $-2.88 \mathrm{E}-04$ & $1.80 \mathrm{E}-01$ & $-1.55 \mathrm{E}-02$ & $4.13 \mathrm{E}+00$ \\
\hline 10000 & 22.0 & $1.56 \mathrm{E}-02$ & $2.42 \mathrm{E}-06$ & $1.87 \mathrm{E}-01$ & $-2.88 \mathrm{E}-04$ & $1.88 \mathrm{E}-01$ & $-1.62 \mathrm{E}-02$ & $4.13 E+00$ \\
\hline 10000 & 21.0 & $1.63 \mathrm{E}-02$ & $9.78 \mathrm{E}-07$ & $1.96 \mathrm{E}-01$ & $-2.88 \mathrm{E}-04$ & $1.96 \mathrm{E}-01$ & $-1.69 \mathrm{E}-02$ & $4.12 \mathrm{E}+00$ \\
\hline 10000 & 20.0 & $1.71 \mathrm{E}-02$ & $8.58 \mathrm{E}-07$ & $2.06 \mathrm{E}-01$ & $-2.88 \mathrm{E}-04$ & $2.06 \mathrm{E}-01$ & $-1.77 \mathrm{E}-02$ & $4.12 \mathrm{E}+00$ \\
\hline 10000 & 19.0 & $1.80 \mathrm{E}-02$ & $5.62 \mathrm{E}-07$ & $2.16 \mathrm{E}-01$ & $-2.88 \mathrm{E}-04$ & $2.17 \mathrm{E}-01$ & $-1.87 \mathrm{E}-02$ & $4.12 \mathrm{E}+00$ \\
\hline 10000 & 18.0 & $1.89 \mathrm{E}-02$ & $1.17 \mathrm{E}-05$ & $2.28 \mathrm{E}-01$ & $-2.88 \mathrm{E}-04$ & $2.28 \mathrm{E}-01$ & $-1.98 \mathrm{E}-02$ & $4.11 \mathrm{E}+00$ \\
\hline 10000 & 17.0 & $2.00 \mathrm{E}-02$ & $2.01 \mathrm{E}-06$ & $2.41 \mathrm{E}-01$ & $-2.88 \mathrm{E}-04$ & $2.42 \mathrm{E}-01$ & $-2.08 \mathrm{E}-02$ & $4.11 \mathrm{E}+00$ \\
\hline 10000 & 16.0 & $2.12 \mathrm{E}-02$ & $1.07 \mathrm{E}-05$ & $2.56 \mathrm{E}-01$ & $-2.88 \mathrm{E}-04$ & $2.56 \mathrm{E}-01$ & $-2.22 \mathrm{E}-02$ & $4.10 \mathrm{E}+00$ \\
\hline
\end{tabular}




$\begin{array}{lllllllll}10000 & 15.0 & 2.26 \mathrm{E}-02 & 8.09 \mathrm{E}-06 & 2.73 \mathrm{E}-01 & -2.88 \mathrm{E}-04 & 2.73 \mathrm{E}-01 & -2.36 \mathrm{E}-02 & 4.10 \mathrm{E}+00 \\ 10000 & 14.0 & 2.42 \mathrm{E}-02 & 7.36 \mathrm{E}-06 & 2.91 \mathrm{E}-01 & -2.88 \mathrm{E}-04 & 2.92 \mathrm{E}-01 & -2.52 \mathrm{E}-02 & 4.09 \mathrm{E}+00 \\ 10000 & 13.0 & 2.60 \mathrm{E}-02 & 7.72 \mathrm{E}-06 & 3.13 \mathrm{E}-01 & -2.88 \mathrm{E}-04 & 3.13 \mathrm{E}-01 & -2.71 \mathrm{E}-02 & 4.07 \mathrm{E}+00 \\ 10000 & 12.0 & 2.80 \mathrm{E}-02 & 5.62 \mathrm{E}-06 & 3.38 \mathrm{E}-01 & -2.88 \mathrm{E}-04 & 3.38 \mathrm{E}-01 & -2.92 \mathrm{E}-02 & 4.05 \mathrm{E}+00 \\ 10000 & 11.0 & 3.05 \mathrm{E}-02 & 1.20 \mathrm{E}-05 & 3.67 \mathrm{E}-01 & -2.88 \mathrm{E}-04 & 3.68 \mathrm{E}-01 & -3.18 \mathrm{E}-02 & 4.05 \mathrm{E}+00 \\ 10000 & 10.0 & 3.34 \mathrm{E}-02 & 2.08 \mathrm{E}-05 & 4.03 \mathrm{E}-01 & -2.88 \mathrm{E}-04 & 4.03 \mathrm{E}-01 & -3.50 \mathrm{E}-02 & 4.03 \mathrm{E}+00 \\ 10000 & 9.0 & 3.69 \mathrm{E}-02 & 3.37 \mathrm{E}-05 & 4.45 \mathrm{E}-01 & -2.88 \mathrm{E}-04 & 4.45 \mathrm{E}-01 & -3.88 \mathrm{E}-02 & 4.01 \mathrm{E}+00 \\ 10000 & 8.0 & 4.13 \mathrm{E}-02 & 2.00 \mathrm{E}-05 & 4.97 \mathrm{E}-01 & -2.88 \mathrm{E}-04 & 4.98 \mathrm{E}-01 & -4.31 \mathrm{E}-02 & 3.98 \mathrm{E}+00 \\ 10000 & 7.0 & 4.67 \mathrm{E}-02 & 5.21 \mathrm{E}-05 & 5.63 \mathrm{E}-01 & -2.88 \mathrm{E}-04 & 5.63 \mathrm{E}-01 & -4.92 \mathrm{E}-02 & 3.95 \mathrm{E}+00 \\ 10000 & 6.0 & 5.37 \mathrm{E}-02 & 6.68 \mathrm{E}-05 & 6.47 \mathrm{E}-01 & -2.88 \mathrm{E}-04 & 6.48 \mathrm{E}-01 & -5.67 \mathrm{E}-02 & 3.89 \mathrm{E}+00 \\ 10000 & 5.0 & 6.30 \mathrm{E}-02 & 4.72 \mathrm{E}-05 & 7.60 \mathrm{E}-01 & -2.88 \mathrm{E}-04 & 7.60 \mathrm{E}-01 & -6.61 \mathrm{E}-02 & 3.80 \mathrm{E}+00\end{array}$


Complex 4 ([\{ $\mathrm{Mn}\left(\mathrm{CH}_{2} \mathrm{CMe}_{3}\right)_{2}(\mu$-dmpe $\left.\left.\left.)\right\}_{2}\right]\right)$

Mass (mg): 57.2

Field

(Oe)

100

100

100

100

100

100

100

100

100

100

100

100

100

100

100

100

100

100

100

100

100

100

100

100

100

100

100

100

100

100

100

100

100

100

100

100

100

$100 \quad 115.0$

$100 \quad 110.0$
Mol. Wt. $\left(\mathrm{gmol}^{-1}\right): 694.72$

\begin{tabular}{|c|c|c|c|c|c|c|c|}
\hline $\operatorname{emp}(\mathrm{K})$ & EMU & STD & $\begin{array}{c}\text { Chi } \\
\text { (cm3/mol) }\end{array}$ & $\begin{array}{c}\text { dia } \\
(\mathrm{cm} 3 / \mathrm{mol})\end{array}$ & $\begin{array}{l}\text { Cor Chi } \\
(\mathrm{cm} 3 / \mathrm{mol})\end{array}$ & esd & $\begin{array}{c}\text { Chi*T } \\
(\mathrm{cm} 3 \mathrm{~K} / \mathrm{mol})\end{array}$ \\
\hline 300.0 & $2.27 \mathrm{E}-04$ & $.52 \mathrm{E}-08$ & $76 \mathrm{E}-02$ & $-5.48 \mathrm{E}-04$ & $2.82 \mathrm{E}-02$ & $-2.45 \mathrm{E}-04$ & $8.45 \mathrm{E}+00$ \\
\hline 294.7 & 2.33E-04 & $2.02 \mathrm{E}-07$ & $2.83 \mathrm{E}-02$ & $-5.48 \mathrm{E}-04$ & $2.88 \mathrm{E}-02$ & $-2.74 \mathrm{E}-04$ & $8.49 \mathrm{E}+00$ \\
\hline 289.7 & 2.37E-04 & $1.08 \mathrm{E}-07$ & $2.88 \mathrm{E}-02$ & $-5.48 \mathrm{E}-04$ & 2.93E-02 & $-2.67 \mathrm{E}-04$ & $8.49 \mathrm{E}+00$ \\
\hline 284.7 & $2.41 \mathrm{E}-04$ & $.19 \mathrm{E}-08$ & $2.93 \mathrm{E}-02$ & $-5.48 \mathrm{E}-04$ & $2.98 \mathrm{E}-02$ & $-2.67 \mathrm{E}-04$ & $8.49 \mathrm{E}+00$ \\
\hline 279.7 & $2.46 \mathrm{E}-04$ & $1.08 \mathrm{E}-07$ & $2.98 \mathrm{E}-02$ & $-5.48 \mathrm{E}-04$ & $3.04 \mathrm{E}-02$ & $-2.76 \mathrm{E}-04$ & $8.49 \mathrm{E}+00$ \\
\hline 274.7 & $2.50 \mathrm{E}-04$ & $1.24 \mathrm{E}-07$ & $3.04 \mathrm{E}-02$ & $-5.48 \mathrm{E}-04$ & 3.09E-02 & $-2.83 \mathrm{E}-04$ & $8.49 \mathrm{E}+00$ \\
\hline 269.7 & $2.55 \mathrm{E}-04$ & $.09 \mathrm{E}-07$ & $3.10 \mathrm{E}-02$ & $-5.48 \mathrm{E}-04$ & $3.15 \mathrm{E}-02$ & $-2.86 \mathrm{E}-04$ & $8.50 \mathrm{E}+00$ \\
\hline 264.7 & $2.60 \mathrm{E}-04$ & $1.23 \mathrm{E}-07$ & $3.16 \mathrm{E}-02$ & $-5.48 \mathrm{E}-04$ & $3.21 \mathrm{E}-02$ & $-2.93 \mathrm{E}-04$ & $8.50 \mathrm{E}+00$ \\
\hline 259.7 & $2.65 \mathrm{E}-04$ & 7.34E-08 & $3.22 \mathrm{E}-02$ & $-5.48 \mathrm{E}-04$ & $3.27 \mathrm{E}-02$ & $-2.93 \mathrm{E}-04$ & $8.50 \mathrm{E}+00$ \\
\hline 254.7 & $2.70 \mathrm{E}-04$ & $8.42 \mathrm{E}-08$ & $3.28 \mathrm{E}-02$ & $-5.48 \mathrm{E}-04$ & $3.34 \mathrm{E}-02$ & $-3.00 \mathrm{E}-04$ & $8.50 \mathrm{E}+00$ \\
\hline 249.7 & $2.76 \mathrm{E}-04$ & $1.48 \mathrm{E}-07$ & $3.35 \mathrm{E}-02$ & $-5.48 \mathrm{E}-04$ & $3.41 \mathrm{E}-02$ & $-3.14 \mathrm{E}-04$ & $8.51 \mathrm{E}+00$ \\
\hline 244.7 & $2.82 \mathrm{E}-04$ & $1.25 \mathrm{E}-07$ & $3.42 \mathrm{E}-02$ & $-5.48 \mathrm{E}-04$ & $3.48 \mathrm{E}-02$ & $-3.17 \mathrm{E}-04$ & $8.51 \mathrm{E}+00$ \\
\hline 239.7 & $88 \mathrm{E}-04$ & $.55 \mathrm{E}-07$ & $3.50 \mathrm{E}-02$ & $-5.48 \mathrm{E}-04$ & $3.55 \mathrm{E}-02$ & $-3.27 \mathrm{E}-04$ & $8.51 \mathrm{E}+00$ \\
\hline 234.7 & $2.94 \mathrm{E}-04$ & $1.39 \mathrm{E}-07$ & $3.57 \mathrm{E}-02$ & $-5.48 \mathrm{E}-04$ & $3.62 \mathrm{E}-02$ & $-3.32 \mathrm{E}-04$ & $8.51 \mathrm{E}+00$ \\
\hline 229.7 & $3.01 \mathrm{E}-04$ & $1.66 \mathrm{E}-07$ & $3.65 \mathrm{E}-02$ & $-5.48 \mathrm{E}-04$ & $3.71 \mathrm{E}-02$ & $-3.42 \mathrm{E}-04$ & $8.51 \mathrm{E}+00$ \\
\hline 224.7 & -04 & $E-07$ & E-02 & $-5.48 \mathrm{E}-04$ & E-02 & -3 & $8.52 \mathrm{E}+00$ \\
\hline 219.7 & $3.15 \mathrm{E}-04$ & $1.86 \mathrm{E}-07$ & $3.82 \mathrm{E}-02$ & $-5.48 \mathrm{E}-04$ & $3.88 \mathrm{E}-02$ & $-3.60 \mathrm{E}-04$ & $8.52 \mathrm{E}+00$ \\
\hline 214.7 & $3.22 \mathrm{E}-04$ & $2.26 \mathrm{E}-07$ & $3.91 \mathrm{E}-02$ & $-5.48 \mathrm{E}-04$ & $3.97 \mathrm{E}-02$ & $-3.73 \mathrm{E}-04$ & $8.52 \mathrm{E}+00$ \\
\hline 209.7 & E-04 & $E-07$ & E-02 & E-04 & $\mathrm{E}-02$ & -3 & $8.53 \mathrm{E}+00$ \\
\hline 204.7 & $3.38 \mathrm{E}-04$ & $2.21 \mathrm{E}-07$ & $4.11 \mathrm{E}-02$ & $-5.48 \mathrm{E}-04$ & 4.17E-02 & $-3.90 \mathrm{E}-04$ & $8.53 \mathrm{E}+00$ \\
\hline 199.7 & $3.47 \mathrm{E}-04$ & $2.80 \mathrm{E}-07$ & $4.22 \mathrm{E}-02$ & $-5.48 \mathrm{E}-04$ & 4.27E-02 & $-4.06 \mathrm{E}-04$ & $8.53 \mathrm{E}+00$ \\
\hline 194.8 & $3.56 \mathrm{E}-04$ & $3.65 \mathrm{E}-07$ & 4.33E-02 & $-5.48 \mathrm{E}-04$ & 4.38E-02 & -4.26 & $8.53 \mathrm{E}+00$ \\
\hline 189.8 & $3.66 \mathrm{E}-04$ & $3.24 \mathrm{E}-07$ & 4.44E-02 & $-5.48 \mathrm{E}-04$ & $4.50 \mathrm{E}-02$ & $-4.31 \mathrm{E}-04$ & $8.53 \mathrm{E}+00$ \\
\hline 184.8 & $3.76 \mathrm{E}-04$ & $3.26 \mathrm{E}-07$ & $4.56 \mathrm{E}-02$ & $-5.48 \mathrm{E}-04$ & $4.62 \mathrm{E}-02$ & $-4.42 \mathrm{E}-04$ & $8.54 \mathrm{E}+00$ \\
\hline 179.8 & $3.86 \mathrm{E}-04$ & $3.58 \mathrm{E}-07$ & 4.69E-02 & $-5.48 \mathrm{E}-04$ & $4.75 \mathrm{E}-02$ & $-4.58 \mathrm{E}-04$ & $8.54 \mathrm{E}+00$ \\
\hline 174.8 & $3.98 \mathrm{E}-04$ & $3.70 \mathrm{E}-07$ & 4.83E-02 & $-5.48 \mathrm{E}-04$ & $4.89 \mathrm{E}-02$ & $-4.71 \mathrm{E}-04$ & $8.54 \mathrm{E}+00$ \\
\hline 169.8 & $4.10 \mathrm{E}-04$ & 4.37E-07 & 4.97E-02 & $-5.48 \mathrm{E}-04$ & $5.03 \mathrm{E}-02$ & $-4.92 \mathrm{E}-04$ & $8.54 \mathrm{E}+00$ \\
\hline 164.8 & $4.22 \mathrm{E}-04$ & $4.24 \mathrm{E}-07$ & $5.13 \mathrm{E}-02$ & $-5.48 \mathrm{E}-04$ & $5.18 \mathrm{E}-02$ & $-5.04 \mathrm{E}-04$ & $8.55 \mathrm{E}+00$ \\
\hline 107.9 & $4.36 \mathrm{E}-04$ & $5.23 \mathrm{E}-07$ & $5.29 \mathrm{E}-02$ & $-5.48 \mathrm{E}-04$ & $5.35 \mathrm{E}-02$ & $-5.31 \mathrm{E}-04$ & $8.55 \mathrm{E}+00$ \\
\hline 154.9 & $4.50 \mathrm{E}-04$ & $5.18 \mathrm{E}-07$ & $5.47 \mathrm{E}-02$ & $-5.48 \mathrm{E}-04$ & $5.52 \mathrm{E}-02$ & $-5.45 E-04$ & $8.55 \mathrm{E}+00$ \\
\hline 149.9 & $4.65 \mathrm{E}-04$ & $5.25 \mathrm{E}-07$ & $5.65 \mathrm{E}-02$ & $-5.48 \mathrm{E}-04$ & $5.71 \mathrm{E}-02$ & $-5.63 \mathrm{E}-04$ & $8.55 \mathrm{E}+00$ \\
\hline 144.9 & $4.82 \mathrm{E}-04$ & $5.79 \mathrm{E}-07$ & $5.85 \mathrm{E}-02$ & $-5.48 \mathrm{E}-04$ & $5.90 \mathrm{E}-02$ & $-5.87 \mathrm{E}-04$ & $8.55 \mathrm{E}+00$ \\
\hline 139.9 & 4.99E-04 & $6.23 \mathrm{E}-07$ & $6.06 \mathrm{E}-02$ & $-5.48 \mathrm{E}-04$ & $6.12 \mathrm{E}-02$ & $-6.11 \mathrm{E}-04$ & $8.56 \mathrm{E}+00$ \\
\hline 134.9 & $5.18 \mathrm{E}-04$ & $6.77 \mathrm{E}-07$ & $6.29 \mathrm{E}-02$ & $-5.48 \mathrm{E}-04$ & $6.34 \mathrm{E}-02$ & $-6.38 \mathrm{E}-04$ & $8.56 \mathrm{E}+00$ \\
\hline 129.9 & $5.38 \mathrm{E}-04$ & $7.26 \mathrm{E}-07$ & $6.53 \mathrm{E}-02$ & $-5.48 \mathrm{E}-04$ & $6.59 \mathrm{E}-02$ & $-6.65 \mathrm{E}-04$ & $8.56 \mathrm{E}+00$ \\
\hline 124.9 & $5.60 \mathrm{E}-04$ & $7.66 \mathrm{E}-07$ & $6.80 \mathrm{E}-02$ & $-5.48 \mathrm{E}-04$ & $6.85 \mathrm{E}-02$ & $-6.93 \mathrm{E}-04$ & $8.56 \mathrm{E}+00$ \\
\hline 120.0 & $5.83 \mathrm{E}-04$ & $7.95 \mathrm{E}-07$ & $7.08 \mathrm{E}-02$ & $-5.48 \mathrm{E}-04$ & $7.14 \mathrm{E}-02$ & $-7.22 \mathrm{E}-04$ & $8.56 \mathrm{E}+00$ \\
\hline 115.0 & $6.09 \mathrm{E}-04$ & $8.85 \mathrm{E}-07$ & 7.39E-02 & $-5.48 \mathrm{E}-04$ & $7.45 \mathrm{E}-02$ & $-7.60 \mathrm{E}-04$ & $8.56 \mathrm{E}+00$ \\
\hline 110.0 & 6.37E-04 & $9.43 \mathrm{E}-07$ & 7.73E-02 & $-5.48 \mathrm{E}-04$ & 7.79E-02 & -7.97E-04 & $8.56 \mathrm{E}+00$ \\
\hline
\end{tabular}




\begin{tabular}{|c|c|c|c|c|c|c|c|c|}
\hline 100 & 105.0 & 6.67E-04 & $1.03 \mathrm{E}-06$ & $8.10 \mathrm{E}-02$ & $-5.48 \mathrm{E}-04$ & $8.16 \mathrm{E}-02$ & $-8.41 \mathrm{E}-04$ & $8.56 \mathrm{E}+00$ \\
\hline 100 & 100.0 & 7.00E-04 & $1.07 \mathrm{E}-06$ & $8.51 \mathrm{E}-02$ & $-5.48 \mathrm{E}-04$ & 8.56E-02 & $-8.81 \mathrm{E}-04$ & $8.56 \mathrm{E}+00$ \\
\hline 100 & 98.9 & 7.06E-04 & $3.67 \mathrm{E}-07$ & $8.57 \mathrm{E}-02$ & $-5.48 \mathrm{E}-04$ & 8.63E-02 & $-8.01 \mathrm{E}-04$ & $8.53 \mathrm{E}+00$ \\
\hline 100 & 97.9 & 7.14E-04 & $5.57 \mathrm{E}-07$ & 8.67E-02 & $-5.48 \mathrm{E}-04$ & 8.73E-02 & $-8.33 \mathrm{E}-04$ & $8.54 \mathrm{E}+00$ \\
\hline 100 & 96.9 & $7.21 \mathrm{E}-04$ & $5.84 \mathrm{E}-07$ & $8.76 \mathrm{E}-02$ & $-5.48 \mathrm{E}-04$ & $8.82 \mathrm{E}-02$ & $-8.44 \mathrm{E}-04$ & $8.54 \mathrm{E}+00$ \\
\hline 100 & 95.9 & 7.29E-04 & $6.18 \mathrm{E}-07$ & $8.85 \mathrm{E}-02$ & $-5.48 \mathrm{E}-04$ & 8.91E-02 & $-8.56 \mathrm{E}-04$ & $8.54 \mathrm{E}+00$ \\
\hline 100 & 94.9 & 7.37E-04 & 6.39E-07 & 8.95E-02 & $-5.48 \mathrm{E}-04$ & $9.00 \mathrm{E}-02$ & $-8.67 \mathrm{E}-04$ & $8.54 \mathrm{E}+00$ \\
\hline 100 & 93.9 & $7.45 \mathrm{E}-04$ & $5.41 \mathrm{E}-07$ & $9.05 \mathrm{E}-02$ & $-5.48 \mathrm{E}-04$ & $9.10 \mathrm{E}-02$ & $-8.64 \mathrm{E}-04$ & $8.54 \mathrm{E}+00$ \\
\hline 100 & 92.9 & 7.53E-04 & $6.94 \mathrm{E}-07$ & $9.15 \mathrm{E}-02$ & $-5.48 \mathrm{E}-04$ & $9.20 \mathrm{E}-02$ & $-8.92 \mathrm{E}-04$ & $8.55 E+00$ \\
\hline 100 & 91.9 & 7.61E-04 & 7.77E-07 & $9.25 \mathrm{E}-02$ & $-5.48 \mathrm{E}-04$ & $9.30 \mathrm{E}-02$ & $-9.11 \mathrm{E}-04$ & $8.55 \mathrm{E}+00$ \\
\hline 100 & 90.9 & $7.70 \mathrm{E}-04$ & 8.09E-07 & $9.35 \mathrm{E}-02$ & $-5.48 \mathrm{E}-04$ & $9.40 \mathrm{E}-02$ & $-9.23 \mathrm{E}-04$ & $8.55 E+00$ \\
\hline 100 & 89.9 & 7.78E-04 & $8.50 \mathrm{E}-07$ & $9.45 \mathrm{E}-02$ & $-5.48 \mathrm{E}-04$ & $9.51 \mathrm{E}-02$ & $-9.38 \mathrm{E}-04$ & $8.55 \mathrm{E}+00$ \\
\hline 100 & 88.9 & 7.87E-04 & $9.19 \mathrm{E}-07$ & $9.56 \mathrm{E}-02$ & $-5.48 \mathrm{E}-04$ & $9.61 \mathrm{E}-02$ & $-9.55 \mathrm{E}-04$ & $8.55 \mathrm{E}+00$ \\
\hline 100 & 87.9 & 7.96E-04 & $9.80 \mathrm{E}-07$ & $9.67 \mathrm{E}-02$ & $-5.48 \mathrm{E}-04$ & $9.72 \mathrm{E}-02$ & $-9.72 \mathrm{E}-04$ & $8.54 \mathrm{E}+00$ \\
\hline 100 & 86.9 & 8.05E-04 & $9.96 \mathrm{E}-07$ & $9.78 \mathrm{E}-02$ & $-5.48 \mathrm{E}-04$ & $9.83 \mathrm{E}-02$ & $-9.84 \mathrm{E}-04$ & $8.54 \mathrm{E}+00$ \\
\hline 100 & 86.0 & 8.13E-04 & $9.83 \mathrm{E}-07$ & $9.88 \mathrm{E}-02$ & $-5.48 \mathrm{E}-04$ & 9.94E-02 & $-9.92 \mathrm{E}-04$ & $8.54 \mathrm{E}+00$ \\
\hline 100 & 84.9 & $8.24 \mathrm{E}-04$ & $1.18 \mathrm{E}-06$ & $1.00 \mathrm{E}-01$ & $-5.48 \mathrm{E}-04$ & $1.01 \mathrm{E}-01$ & $-1.03 \mathrm{E}-03$ & $8.55 \mathrm{E}+00$ \\
\hline 100 & 83.9 & 8.34E-04 & $1.17 \mathrm{E}-06$ & $1.01 \mathrm{E}-01$ & $-5.48 \mathrm{E}-04$ & $1.02 \mathrm{E}-01$ & $-1.04 \mathrm{E}-03$ & $8.55 \mathrm{E}+00$ \\
\hline 100 & 82.9 & 8.44E-04 & $1.24 \mathrm{E}-06$ & $1.02 \mathrm{E}-01$ & $-5.48 \mathrm{E}-04$ & $1.03 \mathrm{E}-01$ & $-1.06 \mathrm{E}-03$ & $8.54 \mathrm{E}+00$ \\
\hline 100 & 81.9 & $8.54 \mathrm{E}-04$ & $1.30 \mathrm{E}-06$ & $1.04 \mathrm{E}-01$ & $-5.48 \mathrm{E}-04$ & $1.04 \mathrm{E}-01$ & $-1.07 \mathrm{E}-03$ & $8.54 \mathrm{E}+00$ \\
\hline 100 & 80.9 & $8.65 \mathrm{E}-04$ & $1.34 \mathrm{E}-06$ & $1.05 \mathrm{E}-01$ & $-5.48 \mathrm{E}-04$ & $1.06 \mathrm{E}-01$ & $-1.09 \mathrm{E}-03$ & $8.54 \mathrm{E}+00$ \\
\hline 100 & 79.9 & $8.75 \mathrm{E}-04$ & $1.40 \mathrm{E}-06$ & $1.06 \mathrm{E}-01$ & $-5.48 \mathrm{E}-04$ & $1.07 \mathrm{E}-01$ & $-1.11 \mathrm{E}-03$ & $8.54 \mathrm{E}+00$ \\
\hline 100 & 78.9 & 8.86E-04 & $1.44 \mathrm{E}-06$ & $1.08 \mathrm{E}-01$ & $-5.48 \mathrm{E}-04$ & $1.08 \mathrm{E}-01$ & $-1.13 \mathrm{E}-03$ & $8.54 \mathrm{E}+00$ \\
\hline 100 & 78.0 & 8.98E-04 & $1.56 \mathrm{E}-06$ & $1.09 \mathrm{E}-01$ & $-5.48 \mathrm{E}-04$ & $1.10 \mathrm{E}-01$ & $-1.15 \mathrm{E}-03$ & $8.54 \mathrm{E}+00$ \\
\hline 100 & 77.0 & $9.09 \mathrm{E}-04$ & $1.53 \mathrm{E}-06$ & $1.10 \mathrm{E}-01$ & $-5.48 \mathrm{E}-04$ & $1.11 \mathrm{E}-01$ & $-1.16 \mathrm{E}-03$ & $8.54 \mathrm{E}+00$ \\
\hline 100 & 76.0 & $9.21 \mathrm{E}-04$ & $1.63 \mathrm{E}-06$ & $1.12 \mathrm{E}-01$ & $-5.48 \mathrm{E}-04$ & $1.12 \mathrm{E}-01$ & $-1.19 \mathrm{E}-03$ & $8.54 \mathrm{E}+00$ \\
\hline 100 & 75.0 & $9.34 \mathrm{E}-04$ & $1.68 \mathrm{E}-06$ & $1.13 \mathrm{E}-01$ & $-5.48 \mathrm{E}-04$ & $1.14 \mathrm{E}-01$ & $-1.21 \mathrm{E}-03$ & $8.54 \mathrm{E}+00$ \\
\hline 100 & 74.0 & $9.46 \mathrm{E}-04$ & $1.70 \mathrm{E}-06$ & $1.15 \mathrm{E}-01$ & $-5.48 \mathrm{E}-04$ & $1.15 \mathrm{E}-01$ & $-1.22 \mathrm{E}-03$ & $8.54 \mathrm{E}+00$ \\
\hline 100 & 73.0 & $9.59 \mathrm{E}-04$ & $1.69 \mathrm{E}-06$ & $1.16 \mathrm{E}-01$ & $-5.48 \mathrm{E}-04$ & $1.17 \mathrm{E}-01$ & $-1.23 \mathrm{E}-03$ & $8.54 \mathrm{E}+00$ \\
\hline 100 & 72.0 & $9.72 \mathrm{E}-04$ & $1.73 \mathrm{E}-06$ & $1.18 \mathrm{E}-01$ & $-5.48 \mathrm{E}-04$ & $1.19 \mathrm{E}-01$ & $-1.25 \mathrm{E}-03$ & $8.54 \mathrm{E}+00$ \\
\hline 100 & 71.0 & $9.86 \mathrm{E}-04$ & $1.82 \mathrm{E}-06$ & $1.20 \mathrm{E}-01$ & $-5.48 \mathrm{E}-04$ & $1.20 \mathrm{E}-01$ & $-1.28 \mathrm{E}-03$ & $8.54 \mathrm{E}+00$ \\
\hline 100 & 70.0 & $1.00 \mathrm{E}-03$ & $1.75 \mathrm{E}-06$ & $1.21 \mathrm{E}-01$ & $-5.48 \mathrm{E}-04$ & $1.22 \mathrm{E}-01$ & $-1.29 \mathrm{E}-03$ & $8.54 \mathrm{E}+00$ \\
\hline 100 & 69.0 & $1.01 \mathrm{E}-03$ & $1.78 \mathrm{E}-06$ & $1.23 \mathrm{E}-01$ & $-5.48 \mathrm{E}-04$ & $1.24 \mathrm{E}-01$ & $-1.30 \mathrm{E}-03$ & $8.53 E+00$ \\
\hline 100 & 68.0 & $1.03 \mathrm{E}-03$ & $1.81 \mathrm{E}-06$ & $1.25 \mathrm{E}-01$ & $-5.48 \mathrm{E}-04$ & $1.25 \mathrm{E}-01$ & $-1.32 \mathrm{E}-03$ & $8.53 \mathrm{E}+00$ \\
\hline 100 & 67.0 & $1.04 \mathrm{E}-03$ & $1.81 \mathrm{E}-06$ & $1.27 \mathrm{E}-01$ & $-5.48 \mathrm{E}-04$ & $1.27 \mathrm{E}-01$ & $-1.34 \mathrm{E}-03$ & $8.53 \mathrm{E}+00$ \\
\hline 100 & 66.0 & $1.06 \mathrm{E}-03$ & $1.86 \mathrm{E}-06$ & $1.29 \mathrm{E}-01$ & $-5.48 \mathrm{E}-04$ & $1.29 \mathrm{E}-01$ & $-1.36 \mathrm{E}-03$ & $8.53 \mathrm{E}+00$ \\
\hline 100 & 65.0 & $1.07 \mathrm{E}-03$ & $1.77 \mathrm{E}-08$ & $1.30 \mathrm{E}-01$ & $-5.48 \mathrm{E}-04$ & $1.31 \mathrm{E}-01$ & $-1.15 \mathrm{E}-03$ & $8.51 \mathrm{E}+00$ \\
\hline 100 & 64.0 & $1.09 \mathrm{E}-03$ & $4.63 \mathrm{E}-08$ & $1.32 \mathrm{E}-01$ & $-5.48 \mathrm{E}-04$ & $1.33 \mathrm{E}-01$ & $-1.17 \mathrm{E}-03$ & $8.51 \mathrm{E}+00$ \\
\hline 100 & 63.0 & $1.11 \mathrm{E}-03$ & $3.01 \mathrm{E}-08$ & $1.34 \mathrm{E}-01$ & $-5.48 \mathrm{E}-04$ & $1.35 \mathrm{E}-01$ & $-1.19 \mathrm{E}-03$ & $8.51 \mathrm{E}+00$ \\
\hline 100 & 62.0 & $1.12 \mathrm{E}-03$ & $5.96 \mathrm{E}-08$ & $1.37 \mathrm{E}-01$ & $-5.48 \mathrm{E}-04$ & $1.37 \mathrm{E}-01$ & $-1.21 \mathrm{E}-03$ & $8.51 \mathrm{E}+00$ \\
\hline 100 & 61.0 & $1.14 \mathrm{E}-03$ & $2.87 \mathrm{E}-08$ & $1.39 \mathrm{E}-01$ & $-5.48 \mathrm{E}-04$ & $1.39 \mathrm{E}-01$ & $-1.23 \mathrm{E}-03$ & $8.51 \mathrm{E}+00$ \\
\hline 100 & 60.0 & $1.16 \mathrm{E}-03$ & $2.97 \mathrm{E}-08$ & $1.41 \mathrm{E}-01$ & $-5.48 \mathrm{E}-04$ & $1.42 \mathrm{E}-01$ & $-1.25 \mathrm{E}-03$ & $8.51 \mathrm{E}+00$ \\
\hline 100 & 59.0 & $1.18 \mathrm{E}-03$ & 5.73E-08 & $1.44 \mathrm{E}-01$ & $-5.48 \mathrm{E}-04$ & $1.44 \mathrm{E}-01$ & $-1.27 \mathrm{E}-03$ & $8.51 \mathrm{E}+00$ \\
\hline
\end{tabular}




\begin{tabular}{|c|c|c|c|c|c|c|c|c|}
\hline 100 & 58.0 & $1.20 \mathrm{E}-03$ & $1.91 \mathrm{E}-08$ & $1.46 \mathrm{E}-01$ & $-5.48 \mathrm{E}-04$ & $1.47 \mathrm{E}-01$ & $-1.29 \mathrm{E}-03$ & $8.51 \mathrm{E}+00$ \\
\hline 100 & 57.0 & $1.22 \mathrm{E}-03$ & $5.20 \mathrm{E}-08$ & 1.49E-01 & $-5.48 \mathrm{E}-04$ & $1.49 \mathrm{E}-01$ & $-1.32 \mathrm{E}-03$ & $8.50 \mathrm{E}+00$ \\
\hline 100 & 56.0 & $1.24 \mathrm{E}-03$ & 8.98E-08 & $1.51 \mathrm{E}-01$ & $-5.48 \mathrm{E}-04$ & $1.52 \mathrm{E}-01$ & $-1.34 \mathrm{E}-03$ & $8.50 \mathrm{E}+00$ \\
\hline 100 & 55.0 & $1.27 \mathrm{E}-03$ & $1.21 \mathrm{E}-07$ & $1.54 \mathrm{E}-01$ & $-5.48 \mathrm{E}-04$ & $1.54 \mathrm{E}-01$ & $-1.37 \mathrm{E}-03$ & $8.50 \mathrm{E}+00$ \\
\hline 100 & 54.0 & $1.29 \mathrm{E}-03$ & $6.25 \mathrm{E}-08$ & $1.57 \mathrm{E}-01$ & $-5.48 \mathrm{E}-04$ & $1.57 \mathrm{E}-01$ & $-1.39 \mathrm{E}-03$ & $8.50 \mathrm{E}+00$ \\
\hline 100 & 53.0 & $1.32 \mathrm{E}-03$ & $6.54 \mathrm{E}-08$ & $1.60 \mathrm{E}-01$ & $-5.48 \mathrm{E}-04$ & $1.60 \mathrm{E}-01$ & $-1.42 \mathrm{E}-03$ & $8.50 \mathrm{E}+00$ \\
\hline 100 & 52.0 & $1.34 \mathrm{E}-03$ & $3.89 \mathrm{E}-07$ & $1.63 \mathrm{E}-01$ & $-5.48 \mathrm{E}-04$ & $1.63 \mathrm{E}-01$ & $-1.48 \mathrm{E}-03$ & $8.50 \mathrm{E}+00$ \\
\hline 100 & 51.0 & $1.37 \mathrm{E}-03$ & $4.10 \mathrm{E}-07$ & $1.66 \mathrm{E}-01$ & $-5.48 \mathrm{E}-04$ & $1.66 \mathrm{E}-01$ & $-1.51 \mathrm{E}-03$ & $8.50 \mathrm{E}+00$ \\
\hline 100 & 50.0 & $1.39 \mathrm{E}-03$ & $3.46 \mathrm{E}-07$ & $1.69 \mathrm{E}-01$ & $-5.48 \mathrm{E}-04$ & $1.70 \mathrm{E}-01$ & $-1.53 \mathrm{E}-03$ & $8.50 \mathrm{E}+00$ \\
\hline 100 & 49.0 & $1.42 \mathrm{E}-03$ & $2.30 \mathrm{E}-07$ & $1.73 \mathrm{E}-01$ & $-5.48 \mathrm{E}-04$ & $1.73 \mathrm{E}-01$ & $-1.55 \mathrm{E}-03$ & $8.50 \mathrm{E}+00$ \\
\hline 100 & 48.0 & $1.45 \mathrm{E}-03$ & $2.09 \mathrm{E}-07$ & $1.76 \mathrm{E}-01$ & $-5.48 \mathrm{E}-04$ & $1.77 \mathrm{E}-01$ & $-1.58 \mathrm{E}-03$ & $8.50 \mathrm{E}+00$ \\
\hline 100 & 47.0 & $1.48 \mathrm{E}-03$ & $1.33 \mathrm{E}-07$ & $1.80 \mathrm{E}-01$ & $-5.48 \mathrm{E}-04$ & $1.81 \mathrm{E}-01$ & $-1.60 \mathrm{E}-03$ & $8.49 \mathrm{E}+00$ \\
\hline 100 & 46.0 & $1.51 \mathrm{E}-03$ & $1.45 \mathrm{E}-07$ & $1.84 \mathrm{E}-01$ & $-5.48 \mathrm{E}-04$ & $1.84 \mathrm{E}-01$ & $-1.64 \mathrm{E}-03$ & $8.49 \mathrm{E}+00$ \\
\hline 100 & 45.0 & $1.55 \mathrm{E}-03$ & $1.84 \mathrm{E}-07$ & $1.88 \mathrm{E}-01$ & $-5.48 \mathrm{E}-04$ & $1.89 \mathrm{E}-01$ & $-1.68 \mathrm{E}-03$ & $8.49 \mathrm{E}+00$ \\
\hline 100 & 44.0 & $1.58 \mathrm{E}-03$ & $1.10 \mathrm{E}-07$ & $1.92 \mathrm{E}-01$ & $-5.48 \mathrm{E}-04$ & $1.93 \mathrm{E}-01$ & $-1.71 \mathrm{E}-03$ & $8.49 \mathrm{E}+00$ \\
\hline 100 & 43.0 & $1.62 \mathrm{E}-03$ & $1.60 \mathrm{E}-07$ & $1.97 \mathrm{E}-01$ & $-5.48 \mathrm{E}-04$ & $1.97 \mathrm{E}-01$ & $-1.75 \mathrm{E}-03$ & $8.48 \mathrm{E}+00$ \\
\hline 100 & 42.0 & $1.66 \mathrm{E}-03$ & $1.56 \mathrm{E}-07$ & $2.01 \mathrm{E}-01$ & $-5.48 \mathrm{E}-04$ & $2.02 \mathrm{E}-01$ & $-1.79 \mathrm{E}-03$ & $8.48 \mathrm{E}+00$ \\
\hline 100 & 41.0 & $1.70 \mathrm{E}-03$ & $8.55 \mathrm{E}-08$ & $2.06 \mathrm{E}-01$ & $-5.48 \mathrm{E}-04$ & $2.07 \mathrm{E}-01$ & $-1.83 \mathrm{E}-03$ & $8.48 \mathrm{E}+00$ \\
\hline 100 & 40.0 & $1.74 \mathrm{E}-03$ & $1.25 \mathrm{E}-07$ & $2.11 \mathrm{E}-01$ & $-5.48 \mathrm{E}-04$ & $2.12 \mathrm{E}-01$ & $-1.88 \mathrm{E}-03$ & $8.48 \mathrm{E}+00$ \\
\hline 100 & 39.0 & $1.78 \mathrm{E}-03$ & $4.16 \mathrm{E}-07$ & $2.17 \mathrm{E}-01$ & $-5.48 \mathrm{E}-04$ & $2.17 \mathrm{E}-01$ & $-1.96 \mathrm{E}-03$ & $8.48 \mathrm{E}+00$ \\
\hline 100 & 38.0 & $1.83 \mathrm{E}-03$ & $6.20 \mathrm{E}-08$ & $2.22 \mathrm{E}-01$ & $-5.48 \mathrm{E}-04$ & $2.23 \mathrm{E}-01$ & $-1.97 \mathrm{E}-03$ & $8.47 \mathrm{E}+00$ \\
\hline 100 & 37.0 & $1.88 \mathrm{E}-03$ & $1.34 \mathrm{E}-07$ & $2.28 \mathrm{E}-01$ & $-5.48 \mathrm{E}-04$ & $2.29 \mathrm{E}-01$ & $-2.03 \mathrm{E}-03$ & $8.47 \mathrm{E}+00$ \\
\hline 100 & 36.0 & $1.93 \mathrm{E}-03$ & $1.23 \mathrm{E}-07$ & $2.35 \mathrm{E}-01$ & $-5.48 \mathrm{E}-04$ & $2.35 \mathrm{E}-01$ & $-2.08 \mathrm{E}-03$ & $8.47 \mathrm{E}+00$ \\
\hline 100 & 35.0 & $1.99 \mathrm{E}-03$ & $2.21 \mathrm{E}-08$ & $2.41 \mathrm{E}-01$ & $-5.48 \mathrm{E}-04$ & $2.42 \mathrm{E}-01$ & $-2.13 \mathrm{E}-03$ & $8.47 \mathrm{E}+00$ \\
\hline 100 & 34.0 & $2.05 \mathrm{E}-03$ & $1.99 \mathrm{E}-07$ & $2.48 \mathrm{E}-01$ & $-5.48 \mathrm{E}-04$ & $2.49 \mathrm{E}-01$ & $-2.22 \mathrm{E}-03$ & $8.46 \mathrm{E}+00$ \\
\hline 100 & 33.0 & $2.11 \mathrm{E}-03$ & $1.70 \mathrm{E}-07$ & $2.56 \mathrm{E}-01$ & $-5.48 \mathrm{E}-04$ & $2.56 \mathrm{E}-01$ & $-2.28 \mathrm{E}-03$ & $8.46 \mathrm{E}+00$ \\
\hline 100 & 32.0 & $2.17 \mathrm{E}-03$ & $2.21 \mathrm{E}-07$ & 2.64E-01 & $-5.48 \mathrm{E}-04$ & $2.64 \mathrm{E}-01$ & $-2.35 \mathrm{E}-03$ & $8.46 \mathrm{E}+00$ \\
\hline 100 & 31.0 & $2.24 \mathrm{E}-03$ & $2.31 \mathrm{E}-07$ & $2.72 \mathrm{E}-01$ & $-5.48 \mathrm{E}-04$ & $2.73 \mathrm{E}-01$ & $-2.43 \mathrm{E}-03$ & $8.46 \mathrm{E}+00$ \\
\hline 100 & 30.0 & $2.32 \mathrm{E}-03$ & $2.83 \mathrm{E}-07$ & $2.81 \mathrm{E}-01$ & $-5.48 \mathrm{E}-04$ & $2.82 \mathrm{E}-01$ & $-2.51 \mathrm{E}-03$ & $8.45 \mathrm{E}+00$ \\
\hline 100 & 29.0 & $2.39 \mathrm{E}-03$ & $3.09 \mathrm{E}-07$ & $2.91 \mathrm{E}-01$ & $-5.48 \mathrm{E}-04$ & $2.91 \mathrm{E}-01$ & $-2.60 \mathrm{E}-03$ & $8.45 \mathrm{E}+00$ \\
\hline 100 & 28.0 & $2.48 \mathrm{E}-03$ & $3.69 \mathrm{E}-07$ & $3.01 \mathrm{E}-01$ & $-5.48 \mathrm{E}-04$ & $3.02 \mathrm{E}-01$ & $-2.70 \mathrm{E}-03$ & $8.44 \mathrm{E}+00$ \\
\hline 100 & 27.0 & $2.57 \mathrm{E}-03$ & $4.26 \mathrm{E}-07$ & $3.12 \mathrm{E}-01$ & $-5.48 \mathrm{E}-04$ & $3.13 \mathrm{E}-01$ & $-2.80 \mathrm{E}-03$ & $8.44 \mathrm{E}+00$ \\
\hline 100 & 26.0 & $2.67 \mathrm{E}-03$ & $3.51 \mathrm{E}-07$ & $3.24 \mathrm{E}-01$ & $-5.48 \mathrm{E}-04$ & $3.24 \mathrm{E}-01$ & $-2.90 \mathrm{E}-03$ & $8.43 \mathrm{E}+00$ \\
\hline 100 & 25.0 & $2.77 \mathrm{E}-03$ & $1.29 \mathrm{E}-06$ & $3.37 \mathrm{E}-01$ & $-5.48 \mathrm{E}-04$ & $3.37 \mathrm{E}-01$ & $-3.13 \mathrm{E}-03$ & $8.43 \mathrm{E}+00$ \\
\hline 100 & 24.0 & $2.89 \mathrm{E}-03$ & $1.60 \mathrm{E}-06$ & $3.50 \mathrm{E}-01$ & $-5.48 \mathrm{E}-04$ & $3.51 \mathrm{E}-01$ & $-3.29 \mathrm{E}-03$ & $8.42 \mathrm{E}+00$ \\
\hline 100 & 23.0 & $3.01 \mathrm{E}-03$ & $6.65 \mathrm{E}-07$ & $3.66 \mathrm{E}-01$ & $-5.48 \mathrm{E}-04$ & $3.66 \mathrm{E}-01$ & $-3.30 \mathrm{E}-03$ & $8.42 \mathrm{E}+00$ \\
\hline 100 & 22.0 & $3.14 \mathrm{E}-03$ & $5.05 \mathrm{E}-07$ & $3.82 \mathrm{E}-01$ & $-5.48 \mathrm{E}-04$ & $3.82 \mathrm{E}-01$ & $-3.43 \mathrm{E}-03$ & $8.41 \mathrm{E}+00$ \\
\hline 100 & 21.0 & $3.29 \mathrm{E}-03$ & $3.34 \mathrm{E}-07$ & 4.00E-01 & $-5.48 \mathrm{E}-04$ & $4.00 \mathrm{E}-01$ & $-3.57 \mathrm{E}-03$ & $8.41 \mathrm{E}+00$ \\
\hline 100 & 20.0 & $3.45 \mathrm{E}-03$ & $1.27 \mathrm{E}-07$ & $4.20 \mathrm{E}-01$ & $-5.48 \mathrm{E}-04$ & $4.20 \mathrm{E}-01$ & $-3.72 \mathrm{E}-03$ & $8.40 \mathrm{E}+00$ \\
\hline 100 & 19.0 & $3.63 \mathrm{E}-03$ & $1.85 \mathrm{E}-06$ & 4.41E-01 & $-5.48 \mathrm{E}-04$ & 4.42E-01 & $-4.12 \mathrm{E}-03$ & $8.40 \mathrm{E}+00$ \\
\hline 100 & 18.0 & $3.83 \mathrm{E}-03$ & $2.21 \mathrm{E}-06$ & 4.65E-01 & $-5.48 \mathrm{E}-04$ & $4.66 \mathrm{E}-01$ & $-4.37 \mathrm{E}-03$ & $8.39 \mathrm{E}+00$ \\
\hline 100 & 17.0 & 4.06E-03 & $1.99 \mathrm{E}-07$ & 4.93E-01 & $-5.48 \mathrm{E}-04$ & $4.93 \mathrm{E}-01$ & $-4.37 \mathrm{E}-03$ & $8.38 \mathrm{E}+00$ \\
\hline 100 & 16.0 & $4.30 \mathrm{E}-03$ & $1.36 \mathrm{E}-06$ & $5.23 \mathrm{E}-01$ & $-5.48 \mathrm{E}-04$ & $5.23 \mathrm{E}-01$ & $-4.78 \mathrm{E}-03$ & $8.37 \mathrm{E}+00$ \\
\hline
\end{tabular}




$\begin{array}{lllllllll}100 & 15.0 & 4.59 \mathrm{E}-03 & 1.16 \mathrm{E}-06 & 5.57 \mathrm{E}-01 & -5.48 \mathrm{E}-04 & 5.57 \mathrm{E}-01 & -5.05 \mathrm{E}-03 & 8.36 \mathrm{E}+00 \\ 100 & 14.0 & 4.90 \mathrm{E}-03 & 1.63 \mathrm{E}-06 & 5.96 \mathrm{E}-01 & -5.48 \mathrm{E}-04 & 5.96 \mathrm{E}-01 & -5.45 \mathrm{E}-03 & 8.35 \mathrm{E}+00 \\ 100 & 13.0 & 5.27 \mathrm{E}-03 & 9.83 \mathrm{E}-07 & 6.40 \mathrm{E}-01 & -5.48 \mathrm{E}-04 & 6.40 \mathrm{E}-01 & -5.76 \mathrm{E}-03 & 8.33 \mathrm{E}+00 \\ 100 & 12.0 & 5.68 \mathrm{E}-03 & 2.95 \mathrm{E}-06 & 6.90 \mathrm{E}-01 & -5.48 \mathrm{E}-04 & 6.91 \mathrm{E}-01 & -6.45 \mathrm{E}-03 & 8.29 \mathrm{E}+00 \\ 100 & 11.0 & 6.20 \mathrm{E}-03 & 3.03 \mathrm{E}-06 & 7.53 \mathrm{E}-01 & -5.48 \mathrm{E}-04 & 7.54 \mathrm{E}-01 & -7.01 \mathrm{E}-03 & 8.30 \mathrm{E}+00 \\ 100 & 10.0 & 6.80 \mathrm{E}-03 & 4.74 \mathrm{E}-06 & 8.26 \mathrm{E}-01 & -5.48 \mathrm{E}-04 & 8.27 \mathrm{E}-01 & -7.87 \mathrm{E}-03 & 8.27 \mathrm{E}+00 \\ 100 & 9.0 & 7.54 \mathrm{E}-03 & 7.05 \mathrm{E}-06 & 9.16 \mathrm{E}-01 & -5.48 \mathrm{E}-04 & 9.16 \mathrm{E}-01 & -8.94 \mathrm{E}-03 & 8.25 \mathrm{E}+00 \\ 100 & 8.0 & 8.45 \mathrm{E}-03 & 3.38 \mathrm{E}-06 & 1.03 \mathrm{E}+00 & -5.48 \mathrm{E}-04 & 1.03 \mathrm{E}+00 & -9.46 \mathrm{E}-03 & 8.22 \mathrm{E}+00 \\ 100 & 7.0 & 9.60 \mathrm{E}-03 & 1.10 \mathrm{E}-05 & 1.17 \mathrm{E}+00 & -5.48 \mathrm{E}-04 & 1.17 \mathrm{E}+00 & -1.16 \mathrm{E}-02 & 8.17 \mathrm{E}+00 \\ 100 & 6.0 & 1.11 \mathrm{E}-02 & 1.31 \mathrm{E}-05 & 1.35 \mathrm{E}+00 & -5.48 \mathrm{E}-04 & 1.35 \mathrm{E}+00 & -1.35 \mathrm{E}-02 & 8.11 \mathrm{E}+00 \\ 100 & 5.0 & 1.32 \mathrm{E}-02 & 1.93 \mathrm{E}-05 & 1.60 \mathrm{E}+00 & -5.48 \mathrm{E}-04 & 1.60 \mathrm{E}+00 & -1.65 \mathrm{E}-02 & 8.00 \mathrm{E}+00\end{array}$


Complex 5 ([ $\left\{\mathrm{Mn}\left(\mathrm{CH}_{2} \mathrm{SiMe}_{3}\right)\left(\mu-\mathrm{CH}_{2} \mathrm{SiMe}_{3}\right)\right\}_{2}(\mu$-dmpe $\left.\left.)\right]\right)$

Mass (mg): 57.6

Field

(Oe)

100

100

100

100

100

100

100

100

100

100

100

100

100

100

100

100

100

100

100

100

100

100

100

100

100

100

100

100

100

100

100

100

100

100

100

100

100

$100 \quad 115.0$

$100 \quad 110.0$
Mol. Wt. $\left(\mathrm{gmol}^{-1}\right): 608.88$

\begin{tabular}{|c|c|c|c|c|c|c|c|}
\hline Temp (K) & EMU & STD & $\begin{array}{c}\text { Chi } \\
(\mathrm{cm} 3 / \mathrm{mol})\end{array}$ & $\begin{array}{c}\mathrm{dia} \\
(\mathrm{cm} 3 / \mathrm{mol})\end{array}$ & $\begin{array}{l}\text { Cor Chi } \\
(\mathrm{cm} 3 / \mathrm{mol})\end{array}$ & esd & $\begin{array}{c}\text { Chi*T } \\
(\mathrm{cm} 3 \mathrm{~K} / \mathrm{mol})\end{array}$ \\
\hline 300.0 & $6.17 \mathrm{E}-05$ & $5.57 \mathrm{E}-08$ & $6.53 \mathrm{E}-03$ & $-4.40 \mathrm{E}-04$ & $6.97 \mathrm{E}-03$ & 5.89E-06 & $2.09 \mathrm{E}+00$ \\
\hline 294.7 & $6.22 \mathrm{E}-05$ & 7.84E-08 & $6.58 \mathrm{E}-03$ & $-4.40 \mathrm{E}-04$ & 7.02E-03 & $8.29 \mathrm{E}-06$ & $2.07 \mathrm{E}+00$ \\
\hline 289.7 & $6.24 \mathrm{E}-05$ & $3.21 \mathrm{E}-08$ & $6.60 \mathrm{E}-03$ & $-4.40 \mathrm{E}-04$ & 7.04E-03 & $3.40 \mathrm{E}-06$ & $2.04 \mathrm{E}+00$ \\
\hline 284.7 & $6.26 \mathrm{E}-05$ & 3.49E-08 & $6.62 \mathrm{E}-03$ & $-4.40 \mathrm{E}-04$ & $7.06 \mathrm{E}-03$ & 3.69E-06 & $2.01 \mathrm{E}+00$ \\
\hline 279.7 & $6.27 \mathrm{E}-05$ & 2.83E-08 & $6.63 \mathrm{E}-03$ & $-4.40 \mathrm{E}-04$ & 7.07E-03 & 2.99E-06 & $1.98 \mathrm{E}+00$ \\
\hline 274.7 & $6.29 \mathrm{E}-05$ & $2.16 \mathrm{E}-08$ & $6.64 \mathrm{E}-03$ & $-4.40 \mathrm{E}-04$ & $7.08 \mathrm{E}-03$ & $2.28 \mathrm{E}-06$ & $1.95 \mathrm{E}+00$ \\
\hline 269.7 & $6.29 \mathrm{E}-05$ & $2.51 \mathrm{E}-08$ & $6.65 \mathrm{E}-03$ & $-4.40 \mathrm{E}-04$ & 7.09E-03 & $.65 \mathrm{E}-06$ & $1.91 \mathrm{E}+00$ \\
\hline 264.7 & $6.30 \mathrm{E}-05$ & $4.75 \mathrm{E}-08$ & $6.66 \mathrm{E}-03$ & $-4.40 \mathrm{E}-04$ & $7.10 \mathrm{E}-03$ & 5.03E-06 & $1.88 \mathrm{E}+00$ \\
\hline 259.7 & $6.30 \mathrm{E}-05$ & 2.04E-08 & $6.66 \mathrm{E}-03$ & $-4.40 \mathrm{E}-04$ & $7.10 \mathrm{E}-03$ & $.16 \mathrm{E}-06$ & $1.84 \mathrm{E}+00$ \\
\hline 254.7 & $6.31 \mathrm{E}-05$ & $1.06 \mathrm{E}-08$ & $6.67 \mathrm{E}-03$ & $-4.40 \mathrm{E}-04$ & $7.11 \mathrm{E}-03$ & $1.12 \mathrm{E}-06$ & $1.81 \mathrm{E}+00$ \\
\hline 249.7 & $6.31 \mathrm{E}-05$ & $1.05 \mathrm{E}-08$ & $6.67 \mathrm{E}-03$ & $-4.40 \mathrm{E}-04$ & $7.11 \mathrm{E}-03$ & $1.11 \mathrm{E}-06$ & $1.78 \mathrm{E}+00$ \\
\hline 244.7 & $6.31 \mathrm{E}-05$ & $3.88 \mathrm{E}-09$ & $6.67 \mathrm{E}-03$ & $-4.40 \mathrm{E}-04$ & $7.11 \mathrm{E}-03$ & 4.10E-07 & $1.74 \mathrm{E}+00$ \\
\hline 239.7 & $6.31 \mathrm{E}-05$ & $1.56 \mathrm{E}-08$ & $6.67 \mathrm{E}-03$ & $-4.40 \mathrm{E}-04$ & $7.11 \mathrm{E}-03$ & $.65 \mathrm{E}-06$ & $1.70 \mathrm{E}+00$ \\
\hline 234.7 & $6.30 \mathrm{E}-05$ & $9.96 \mathrm{E}-09$ & $6.66 \mathrm{E}-03$ & $-4.40 \mathrm{E}-04$ & 7.10E-03 & $1.05 \mathrm{E}-06$ & $1.67 \mathrm{E}+00$ \\
\hline 229.7 & $6.30 \mathrm{E}-05$ & $6.86 \mathrm{E}-09$ & $6.66 \mathrm{E}-03$ & $-4.40 \mathrm{E}-04$ & $7.10 \mathrm{E}-03$ & $7.25 \mathrm{E}-07$ & $1.63 \mathrm{E}+00$ \\
\hline 224.7 & $6.29 \mathrm{E}-05$ & 4.86E-09 & $6.65 \mathrm{E}-03$ & $-4.40 \mathrm{E}-04$ & 7.09E-03 & 14E-07 & $1.59 \mathrm{E}+00$ \\
\hline 219.8 & $6.28 \mathrm{E}-05$ & $3.32 \mathrm{E}-09$ & $6.64 \mathrm{E}-03$ & $-4.40 \mathrm{E}-04$ & $7.08 \mathrm{E}-03$ & $3.51 \mathrm{E}-07$ & $1.56 \mathrm{E}+00$ \\
\hline 214.7 & $6.28 \mathrm{E}-05$ & $1.80 \mathrm{E}-08$ & $6.64 \mathrm{E}-03$ & $-4.40 \mathrm{E}-04$ & $7.08 \mathrm{E}-03$ & $1.90 \mathrm{E}-06$ & $1.52 \mathrm{E}+00$ \\
\hline 209.7 & $6.27 \mathrm{E}-05$ & $1.07 \mathrm{E}-08$ & $6.63 \mathrm{E}-03$ & $-4.40 \mathrm{E}-04$ & 7.07E-03 & $.14 \mathrm{E}-06$ & $1.48 \mathrm{E}+00$ \\
\hline 204.7 & $6.27 \mathrm{E}-05$ & $1.51 \mathrm{E}-09$ & $6.63 \mathrm{E}-03$ & $-4.40 \mathrm{E}-04$ & 7.07E-03 & $1.59 \mathrm{E}-07$ & $1.45 \mathrm{E}+00$ \\
\hline 199.7 & $6.26 \mathrm{E}-05$ & 5.77E-09 & $6.62 \mathrm{E}-03$ & $-4.40 \mathrm{E}-04$ & $7.06 \mathrm{E}-03$ & $6.10 \mathrm{E}-07$ & $1.41 \mathrm{E}+00$ \\
\hline 194.7 & $6.25 \mathrm{E}-05$ & $3.25 \mathrm{E}-09$ & $6.61 \mathrm{E}-03$ & $-4.40 \mathrm{E}-04$ & $7.05 \mathrm{E}-03$ & $3.43 \mathrm{E}-07$ & $1.37 \mathrm{E}+00$ \\
\hline 189.8 & $6.25 \mathrm{E}-05$ & $9.23 \mathrm{E}-09$ & $6.61 \mathrm{E}-03$ & $-4.40 \mathrm{E}-04$ & 7.05E-03 & $.76 \mathrm{E}-07$ & $1.34 \mathrm{E}+00$ \\
\hline 184.8 & $6.24 \mathrm{E}-05$ & $5.77 \mathrm{E}-09$ & $6.60 \mathrm{E}-03$ & $-4.40 \mathrm{E}-04$ & 7.04E-03 & $6.10 \mathrm{E}-07$ & $1.30 \mathrm{E}+00$ \\
\hline 179.8 & $6.24 \mathrm{E}-05$ & $6.00 \mathrm{E}-09$ & $6.59 \mathrm{E}-03$ & $-4.40 \mathrm{E}-04$ & 7.03E-03 & $6.34 \mathrm{E}-07$ & $1.26 \mathrm{E}+00$ \\
\hline 174.8 & $6.23 \mathrm{E}-05$ & 9.84E-09 & $6.59 \mathrm{E}-03$ & $-4.40 \mathrm{E}-04$ & 7.03E-03 & $1.04 \mathrm{E}-06$ & $1.23 \mathrm{E}+00$ \\
\hline 169.8 & $6.23 \mathrm{E}-05$ & $1.59 \mathrm{E}-08$ & $6.59 \mathrm{E}-03$ & $-4.40 \mathrm{E}-04$ & $7.03 \mathrm{E}-03$ & $1.68 \mathrm{E}-06$ & $1.19 \mathrm{E}+00$ \\
\hline 164.8 & $6.23 \mathrm{E}-05$ & 4.04E-09 & $6.58 \mathrm{E}-03$ & $-4.40 \mathrm{E}-04$ & 7.02E-03 & 4.27E-07 & $1.16 \mathrm{E}+00$ \\
\hline 159.8 & $6.22 \mathrm{E}-05$ & 2.89E-08 & $6.57 \mathrm{E}-03$ & $-4.40 \mathrm{E}-04$ & $7.01 \mathrm{E}-03$ & $3.06 \mathrm{E}-06$ & $1.12 \mathrm{E}+00$ \\
\hline 154.9 & $6.22 \mathrm{E}-05$ & 9.59E-09 & $6.58 \mathrm{E}-03$ & $-4.40 \mathrm{E}-04$ & $7.02 \mathrm{E}-03$ & $1.01 \mathrm{E}-06$ & $1.09 \mathrm{E}+00$ \\
\hline 149.9 & $6.22 \mathrm{E}-05$ & 5.97E-09 & $6.58 \mathrm{E}-03$ & $-4.40 \mathrm{E}-04$ & 7.02E-03 & $6.31 \mathrm{E}-07$ & $1.05 \mathrm{E}+00$ \\
\hline 144.9 & $6.22 \mathrm{E}-05$ & 4.18E-09 & $6.57 \mathrm{E}-03$ & $-4.40 \mathrm{E}-04$ & $7.01 \mathrm{E}-03$ & 4.42E-07 & $1.02 \mathrm{E}+00$ \\
\hline 139.9 & $6.21 \mathrm{E}-05$ & 4.23E-09 & $6.57 \mathrm{E}-03$ & $-4.40 \mathrm{E}-04$ & 7.01E-03 & 4.47E-07 & $9.80 \mathrm{E}-01$ \\
\hline 134.9 & $6.21 \mathrm{E}-05$ & 4.07E-09 & $6.56 \mathrm{E}-03$ & $-4.40 \mathrm{E}-04$ & $7.00 \mathrm{E}-03$ & 4.30E-07 & $9.45 \mathrm{E}-01$ \\
\hline 129.9 & $6.21 \mathrm{E}-05$ & 7.95E-09 & $6.56 \mathrm{E}-03$ & $-4.40 \mathrm{E}-04$ & $7.00 \mathrm{E}-03$ & 8.40E-07 & $9.09 \mathrm{E}-01$ \\
\hline 124.9 & $6.20 \mathrm{E}-05$ & 5.99E-09 & $6.55 \mathrm{E}-03$ & $-4.40 \mathrm{E}-04$ & $6.99 \mathrm{E}-03$ & $6.33 \mathrm{E}-07$ & $8.74 \mathrm{E}-01$ \\
\hline 119.9 & $6.18 \mathrm{E}-05$ & $6.36 \mathrm{E}-09$ & $6.54 \mathrm{E}-03$ & $-4.40 \mathrm{E}-04$ & $6.98 \mathrm{E}-03$ & $6.73 \mathrm{E}-07$ & 8.37E-01 \\
\hline 115.0 & $6.16 \mathrm{E}-05$ & $1.70 \mathrm{E}-08$ & $6.51 \mathrm{E}-03$ & $-4.40 \mathrm{E}-04$ & $6.95 \mathrm{E}-03$ & $1.80 \mathrm{E}-06$ & 7.99E-01 \\
\hline 110.0 & $6.13 \mathrm{E}-05$ & $2.68 \mathrm{E}-08$ & $6.48 \mathrm{E}-03$ & $-4.40 \mathrm{E}-04$ & $6.92 \mathrm{E}-03$ & $2.84 \mathrm{E}-06$ & 7.61E-01 \\
\hline
\end{tabular}




\begin{tabular}{|c|c|c|c|c|c|c|c|c|}
\hline 100 & 105.0 & $6.13 \mathrm{E}-05$ & $1.17 \mathrm{E}-08$ & $6.48 \mathrm{E}-03$ & $-4.40 \mathrm{E}-04$ & $6.92 \mathrm{E}-03$ & $1.24 \mathrm{E}-06$ & 7.26E-01 \\
\hline 100 & 100.0 & $15 \mathrm{E}-05$ & $9.53 \mathrm{E}-09$ & $6.50 \mathrm{E}-03$ & $-4.40 \mathrm{E}-04$ & $6.94 \mathrm{E}-03$ & $1.01 \mathrm{E}-06$ & $6.94 \mathrm{E}-01$ \\
\hline 100 & 99.0 & $6.15 \mathrm{E}-05$ & $9.07 \mathrm{E}-09$ & $6.50 \mathrm{E}-03$ & $-4.40 \mathrm{E}-04$ & $6.94 \mathrm{E}-03$ & $9.59 \mathrm{E}-07$ & $6.87 \mathrm{E}-01$ \\
\hline 100 & 97.9 & $6.15 \mathrm{E}-05$ & 7.02E-09 & $6.51 \mathrm{E}-03$ & $-4.40 \mathrm{E}-04$ & $6.95 \mathrm{E}-03$ & 7.42E-07 & $6.80 \mathrm{E}-01$ \\
\hline 100 & 96.9 & $6.16 \mathrm{E}-05$ & $3.01 \mathrm{E}-09$ & $6.51 \mathrm{E}-03$ & $-4.40 \mathrm{E}-04$ & $6.95 \mathrm{E}-03$ & $3.18 \mathrm{E}-07$ & $6.73 \mathrm{E}-01$ \\
\hline 100 & 95.9 & $6.16 \mathrm{E}-05$ & $6.24 \mathrm{E}-10$ & $6.51 \mathrm{E}-03$ & $-4.40 \mathrm{E}-04$ & $6.95 \mathrm{E}-03$ & $6.60 \mathrm{E}-08$ & 6.67E-01 \\
\hline 100 & 94.9 & $6.16 \mathrm{E}-05$ & $9.48 \mathrm{E}-10$ & $6.52 \mathrm{E}-03$ & $-4.40 \mathrm{E}-04$ & $6.96 \mathrm{E}-03$ & $1.00 \mathrm{E}-07$ & $6.60 \mathrm{E}-01$ \\
\hline 100 & 93.9 & $6.17 \mathrm{E}-05$ & $7.90 \mathrm{E}-09$ & $6.52 \mathrm{E}-03$ & $-4.40 \mathrm{E}-04$ & $6.96 \mathrm{E}-03$ & 8.35E-07 & $6.53 \mathrm{E}-01$ \\
\hline 100 & 92.9 & $6.17 \mathrm{E}-05$ & 8.84E-09 & $6.52 \mathrm{E}-03$ & $-4.40 \mathrm{E}-04$ & $6.96 \mathrm{E}-03$ & 9.34E-07 & $6.47 \mathrm{E}-01$ \\
\hline 100 & 91.9 & $6.35 \mathrm{E}-05$ & $3.15 \mathrm{E}-06$ & $6.72 \mathrm{E}-03$ & $-4.40 \mathrm{E}-04$ & $7.16 \mathrm{E}-03$ & 3.33E-04 & $6.58 \mathrm{E}-01$ \\
\hline 100 & 90.9 & $6.17 \mathrm{E}-05$ & 6.93E-09 & $6.53 \mathrm{E}-03$ & $-4.40 \mathrm{E}-04$ & $6.97 \mathrm{E}-03$ & 7.32E-07 & $6.33 \mathrm{E}-01$ \\
\hline 100 & 89.9 & $6.18 \mathrm{E}-05$ & $8.58 \mathrm{E}-10$ & $6.53 \mathrm{E}-03$ & $-4.40 \mathrm{E}-04$ & $6.97 \mathrm{E}-03$ & $9.07 \mathrm{E}-08$ & $6.27 \mathrm{E}-01$ \\
\hline 100 & 88.9 & $6.18 \mathrm{E}-05$ & 7.78E-09 & $6.53 \mathrm{E}-03$ & $-4.40 \mathrm{E}-04$ & $6.97 \mathrm{E}-03$ & $8.22 \mathrm{E}-07$ & $6.20 \mathrm{E}-01$ \\
\hline 100 & 87.9 & $6.18 \mathrm{E}-05$ & 7.14E-09 & $6.53 \mathrm{E}-03$ & $-4.40 \mathrm{E}-04$ & $6.97 \mathrm{E}-03$ & $7.55 \mathrm{E}-07$ & $6.13 \mathrm{E}-01$ \\
\hline 100 & 86.9 & $6.18 \mathrm{E}-05$ & $8.27 \mathrm{E}-09$ & $6.53 \mathrm{E}-03$ & $-4.40 \mathrm{E}-04$ & $6.97 \mathrm{E}-03$ & $8.75 \mathrm{E}-07$ & $6.06 \mathrm{E}-01$ \\
\hline 100 & 85.9 & $6.18 \mathrm{E}-05$ & 3.57E-09 & $6.54 \mathrm{E}-03$ & $-4.40 \mathrm{E}-04$ & $6.98 \mathrm{E}-03$ & $3.78 \mathrm{E}-07$ & $5.99 \mathrm{E}-01$ \\
\hline 100 & 84.9 & $6.18 \mathrm{E}-05$ & 4.73E-09 & $6.54 \mathrm{E}-03$ & $-4.40 \mathrm{E}-04$ & $6.98 \mathrm{E}-03$ & $5.00 \mathrm{E}-07$ & $5.92 \mathrm{E}-01$ \\
\hline 100 & 83.9 & $6.18 \mathrm{E}-05$ & 4.03E-09 & $6.54 \mathrm{E}-03$ & $-4.40 \mathrm{E}-04$ & $6.98 \mathrm{E}-03$ & $4.26 \mathrm{E}-07$ & $5.86 \mathrm{E}-01$ \\
\hline 100 & 82.9 & $6.19 \mathrm{E}-05$ & $9.21 \mathrm{E}-10$ & $6.54 \mathrm{E}-03$ & $-4.40 \mathrm{E}-04$ & $6.98 \mathrm{E}-03$ & $9.73 \mathrm{E}-08$ & $5.79 \mathrm{E}-01$ \\
\hline 100 & 81.9 & $6.19 \mathrm{E}-05$ & $6.27 \mathrm{E}-09$ & $6.54 \mathrm{E}-03$ & $-4.40 \mathrm{E}-04$ & $6.98 \mathrm{E}-03$ & $6.63 \mathrm{E}-07$ & 5.72E-01 \\
\hline 100 & 80.9 & $6.19 \mathrm{E}-05$ & 4.54E-09 & $6.54 \mathrm{E}-03$ & $-4.40 \mathrm{E}-04$ & $6.98 \mathrm{E}-03$ & $4.80 \mathrm{E}-07$ & $5.65 \mathrm{E}-01$ \\
\hline 100 & 79.9 & $6.19 \mathrm{E}-05$ & $6.87 \mathrm{E}-09$ & $6.54 \mathrm{E}-03$ & $-4.40 \mathrm{E}-04$ & $6.98 \mathrm{E}-03$ & $7.26 \mathrm{E}-07$ & $5.58 \mathrm{E}-01$ \\
\hline 100 & 78.9 & $6.19 \mathrm{E}-05$ & $2.20 \mathrm{E}-09$ & $6.54 \mathrm{E}-03$ & $-4.40 \mathrm{E}-04$ & $6.98 \mathrm{E}-03$ & $2.33 \mathrm{E}-07$ & $5.51 \mathrm{E}-01$ \\
\hline 100 & 78.0 & $6.19 \mathrm{E}-05$ & 1.37E-09 & $6.54 \mathrm{E}-03$ & $-4.40 \mathrm{E}-04$ & $6.98 \mathrm{E}-03$ & $1.44 \mathrm{E}-07$ & $5.44 \mathrm{E}-01$ \\
\hline 100 & 77.0 & $6.19 \mathrm{E}-05$ & 7.14E-09 & $6.54 \mathrm{E}-03$ & $-4.40 \mathrm{E}-04$ & $6.98 \mathrm{E}-03$ & 7.54E-07 & $5.38 \mathrm{E}-01$ \\
\hline 100 & 76.0 & $6.19 \mathrm{E}-05$ & $1.20 \mathrm{E}-08$ & $6.54 \mathrm{E}-03$ & $-4.40 \mathrm{E}-04$ & $6.98 \mathrm{E}-03$ & $1.27 \mathrm{E}-06$ & $5.31 \mathrm{E}-01$ \\
\hline 100 & 75.0 & $6.19 \mathrm{E}-05$ & 7.66E-09 & $6.54 \mathrm{E}-03$ & $-4.40 \mathrm{E}-04$ & $6.99 \mathrm{E}-03$ & $8.10 \mathrm{E}-07$ & $5.24 \mathrm{E}-01$ \\
\hline 100 & 74.0 & $6.19 \mathrm{E}-05$ & 8.67E-09 & $6.54 \mathrm{E}-03$ & $-4.40 \mathrm{E}-04$ & $6.98 \mathrm{E}-03$ & $9.16 \mathrm{E}-07$ & $5.17 \mathrm{E}-01$ \\
\hline 100 & 73.0 & $6.19 \mathrm{E}-05$ & $6.36 \mathrm{E}-09$ & $6.54 \mathrm{E}-03$ & $-4.40 \mathrm{E}-04$ & $6.98 \mathrm{E}-03$ & $6.72 \mathrm{E}-07$ & $5.10 \mathrm{E}-01$ \\
\hline 100 & 72.0 & $6.19 \mathrm{E}-05$ & 2.22E-09 & $6.54 \mathrm{E}-03$ & $-4.40 \mathrm{E}-04$ & $6.98 \mathrm{E}-03$ & $2.35 \mathrm{E}-07$ & 5.03E-01 \\
\hline 100 & 71.0 & $6.19 \mathrm{E}-05$ & $5.75 \mathrm{E}-09$ & $6.54 \mathrm{E}-03$ & $-4.40 \mathrm{E}-04$ & $6.98 \mathrm{E}-03$ & $6.08 \mathrm{E}-07$ & 4.96E-01 \\
\hline 100 & 70.0 & $6.19 \mathrm{E}-05$ & 7.51E-09 & $6.54 \mathrm{E}-03$ & $-4.40 \mathrm{E}-04$ & $6.98 \mathrm{E}-03$ & 7.94E-07 & 4.89E-01 \\
\hline 100 & 69.0 & $6.19 \mathrm{E}-05$ & 8.31E-09 & $6.54 \mathrm{E}-03$ & $-4.40 \mathrm{E}-04$ & $6.98 \mathrm{E}-03$ & $8.78 \mathrm{E}-07$ & $4.82 \mathrm{E}-01$ \\
\hline 100 & 68.0 & $6.19 \mathrm{E}-05$ & 4.92E-09 & $6.54 \mathrm{E}-03$ & $-4.40 \mathrm{E}-04$ & $6.98 \mathrm{E}-03$ & $5.21 \mathrm{E}-07$ & 4.75E-01 \\
\hline 100 & 67.0 & $6.19 \mathrm{E}-05$ & 4.45E-09 & $6.54 \mathrm{E}-03$ & $-4.40 \mathrm{E}-04$ & $6.98 \mathrm{E}-03$ & $4.71 \mathrm{E}-07$ & $4.68 \mathrm{E}-01$ \\
\hline 100 & 66.0 & $6.19 \mathrm{E}-05$ & $3.42 \mathrm{E}-09$ & $6.54 \mathrm{E}-03$ & $-4.40 \mathrm{E}-04$ & $6.98 \mathrm{E}-03$ & $3.61 \mathrm{E}-07$ & 4.61E-01 \\
\hline 100 & 65.0 & $6.18 \mathrm{E}-05$ & $6.33 \mathrm{E}-09$ & $6.54 \mathrm{E}-03$ & $-4.40 \mathrm{E}-04$ & $6.98 \mathrm{E}-03$ & $6.69 \mathrm{E}-07$ & 4.54E-01 \\
\hline 100 & 64.0 & $6.18 \mathrm{E}-05$ & 3.83E-09 & $6.54 \mathrm{E}-03$ & $-4.40 \mathrm{E}-04$ & $6.98 \mathrm{E}-03$ & $4.05 \mathrm{E}-07$ & 4.47E-01 \\
\hline 100 & 63.0 & $6.18 \mathrm{E}-05$ & 2.71E-09 & $6.53 \mathrm{E}-03$ & $-4.40 \mathrm{E}-04$ & $6.97 \mathrm{E}-03$ & $2.86 \mathrm{E}-07$ & 4.40E-01 \\
\hline 100 & 62.0 & $6.18 \mathrm{E}-05$ & $3.21 \mathrm{E}-09$ & $6.53 \mathrm{E}-03$ & $-4.40 \mathrm{E}-04$ & $6.97 \mathrm{E}-03$ & $3.39 \mathrm{E}-07$ & 4.33E-01 \\
\hline 100 & 61.0 & $6.18 \mathrm{E}-05$ & $1.31 \mathrm{E}-10$ & $6.53 \mathrm{E}-03$ & $-4.40 \mathrm{E}-04$ & $6.97 \mathrm{E}-03$ & $1.39 \mathrm{E}-08$ & $4.25 \mathrm{E}-01$ \\
\hline 100 & 60.0 & $6.18 \mathrm{E}-05$ & 2.94E-09 & $6.53 \mathrm{E}-03$ & $-4.40 \mathrm{E}-04$ & $6.97 \mathrm{E}-03$ & $3.11 \mathrm{E}-07$ & $4.18 \mathrm{E}-01$ \\
\hline 100 & 59.0 & $6.18 \mathrm{E}-05$ & $2.66 \mathrm{E}-09$ & $6.53 \mathrm{E}-03$ & $-4.40 \mathrm{E}-04$ & $6.97 \mathrm{E}-03$ & $2.82 \mathrm{E}-07$ & $4.11 \mathrm{E}-01$ \\
\hline
\end{tabular}




\begin{tabular}{|c|c|c|c|c|c|c|c|c|}
\hline 100 & 58.0 & $6.17 \mathrm{E}-05$ & 4.15E-09 & $6.53 \mathrm{E}-03$ & $-4.40 \mathrm{E}-04$ & $6.97 \mathrm{E}-03$ & 4.39E-07 & $4.04 \mathrm{E}-01$ \\
\hline 100 & 57.0 & $6.17 \mathrm{E}-05$ & 7.22E-09 & $6.53 \mathrm{E}-03$ & $-4.40 \mathrm{E}-04$ & $6.97 \mathrm{E}-03$ & 7.64E-07 & $3.97 \mathrm{E}-01$ \\
\hline 100 & 56.0 & $6.17 \mathrm{E}-05$ & 4.69E-09 & $6.52 \mathrm{E}-03$ & $-4.40 \mathrm{E}-04$ & $6.96 \mathrm{E}-03$ & $4.96 \mathrm{E}-07$ & $3.90 \mathrm{E}-01$ \\
\hline 100 & 55.0 & 6.17E-05 & 1.91E-09 & $6.52 \mathrm{E}-03$ & $-4.40 \mathrm{E}-04$ & $6.96 \mathrm{E}-03$ & $2.02 \mathrm{E}-07$ & $3.83 \mathrm{E}-01$ \\
\hline 100 & 54.0 & $6.17 \mathrm{E}-05$ & 6.34E-09 & $6.52 \mathrm{E}-03$ & $-4.40 \mathrm{E}-04$ & $6.96 \mathrm{E}-03$ & $6.70 \mathrm{E}-07$ & $3.76 \mathrm{E}-01$ \\
\hline 100 & 53.0 & $6.17 \mathrm{E}-05$ & 8.17E-09 & $6.52 \mathrm{E}-03$ & $-4.40 \mathrm{E}-04$ & $6.96 \mathrm{E}-03$ & 8.64E-07 & $3.69 \mathrm{E}-01$ \\
\hline 100 & 52.0 & $6.17 \mathrm{E}-05$ & 6.57E-09 & $6.52 \mathrm{E}-03$ & $-4.40 \mathrm{E}-04$ & $6.96 \mathrm{E}-03$ & $6.94 \mathrm{E}-07$ & $3.62 \mathrm{E}-01$ \\
\hline 100 & 51.0 & $6.17 \mathrm{E}-05$ & 4.09E-09 & $6.52 \mathrm{E}-03$ & $-4.40 \mathrm{E}-04$ & $6.96 \mathrm{E}-03$ & 4.32E-07 & $3.55 \mathrm{E}-01$ \\
\hline 100 & 50.0 & $6.17 \mathrm{E}-05$ & 8.46E-09 & $6.52 \mathrm{E}-03$ & $-4.40 \mathrm{E}-04$ & $6.96 \mathrm{E}-03$ & 8.94E-07 & $3.49 \mathrm{E}-01$ \\
\hline 100 & 49.0 & $6.17 \mathrm{E}-05$ & 4.29E-09 & $6.53 \mathrm{E}-03$ & $-4.40 \mathrm{E}-04$ & $6.97 \mathrm{E}-03$ & 4.54E-07 & $3.42 \mathrm{E}-01$ \\
\hline 100 & 48.0 & $6.18 \mathrm{E}-05$ & 4.00E-09 & $6.53 \mathrm{E}-03$ & $-4.40 \mathrm{E}-04$ & $6.97 \mathrm{E}-03$ & 4.23E-07 & $3.35 \mathrm{E}-01$ \\
\hline 100 & 47.0 & $6.18 \mathrm{E}-05$ & 5.59E-09 & $6.54 \mathrm{E}-03$ & $-4.40 \mathrm{E}-04$ & $6.98 \mathrm{E}-03$ & 5.91E-07 & $3.28 \mathrm{E}-01$ \\
\hline 100 & 46.0 & $6.19 \mathrm{E}-05$ & $3.22 \mathrm{E}-09$ & $6.55 \mathrm{E}-03$ & $-4.40 \mathrm{E}-04$ & $6.99 \mathrm{E}-03$ & $3.40 \mathrm{E}-07$ & $3.22 \mathrm{E}-01$ \\
\hline 100 & 45.0 & $6.20 \mathrm{E}-05$ & 4.14E-09 & $6.55 \mathrm{E}-03$ & $-4.40 \mathrm{E}-04$ & $7.00 \mathrm{E}-03$ & 4.38E-07 & $3.15 \mathrm{E}-01$ \\
\hline 100 & 44.0 & $6.21 \mathrm{E}-05$ & $5.58 \mathrm{E}-09$ & $6.57 \mathrm{E}-03$ & $-4.40 \mathrm{E}-04$ & 7.01E-03 & $5.90 \mathrm{E}-07$ & $3.08 \mathrm{E}-01$ \\
\hline 100 & 43.0 & $6.23 \mathrm{E}-05$ & 3.50E-09 & $6.58 \mathrm{E}-03$ & $-4.40 \mathrm{E}-04$ & 7.02E-03 & $3.69 \mathrm{E}-07$ & $3.02 \mathrm{E}-01$ \\
\hline 100 & 42.0 & $6.24 \mathrm{E}-05$ & 2.07E-09 & $6.60 \mathrm{E}-03$ & $-4.40 \mathrm{E}-04$ & 7.04E-03 & 2.19E-07 & $2.96 \mathrm{E}-01$ \\
\hline 100 & 41.0 & $6.26 \mathrm{E}-05$ & $9.81 \mathrm{E}-10$ & $6.62 \mathrm{E}-03$ & $-4.40 \mathrm{E}-04$ & $7.06 \mathrm{E}-03$ & $1.04 \mathrm{E}-07$ & $2.90 \mathrm{E}-01$ \\
\hline 100 & 40.0 & $6.29 \mathrm{E}-05$ & 3.05E-09 & $6.65 \mathrm{E}-03$ & $-4.40 \mathrm{E}-04$ & 7.09E-03 & $3.23 \mathrm{E}-07$ & 2.84E-01 \\
\hline 100 & 39.0 & $6.32 \mathrm{E}-05$ & 8.77E-09 & $6.68 \mathrm{E}-03$ & $-4.40 \mathrm{E}-04$ & 7.12E-03 & $9.27 \mathrm{E}-07$ & $2.78 \mathrm{E}-01$ \\
\hline 100 & 38.0 & $6.35 \mathrm{E}-05$ & $3.43 \mathrm{E}-09$ & $6.71 \mathrm{E}-03$ & $-4.40 \mathrm{E}-04$ & $7.15 \mathrm{E}-03$ & $3.63 \mathrm{E}-07$ & $2.72 \mathrm{E}-01$ \\
\hline 100 & 37.0 & $6.39 \mathrm{E}-05$ & $1.42 \mathrm{E}-09$ & $6.75 \mathrm{E}-03$ & $-4.40 \mathrm{E}-04$ & 7.19E-03 & $1.50 \mathrm{E}-07$ & $2.66 \mathrm{E}-01$ \\
\hline 100 & 36.0 & $6.44 \mathrm{E}-05$ & $5.76 \mathrm{E}-09$ & $6.80 \mathrm{E}-03$ & $-4.40 \mathrm{E}-04$ & 7.24E-03 & $6.09 \mathrm{E}-07$ & $2.61 \mathrm{E}-01$ \\
\hline 100 & 35.0 & $6.49 \mathrm{E}-05$ & 7.69E-09 & $6.86 \mathrm{E}-03$ & $-4.40 \mathrm{E}-04$ & $7.30 \mathrm{E}-03$ & $8.12 \mathrm{E}-07$ & $2.56 \mathrm{E}-01$ \\
\hline 100 & 34.0 & $6.55 \mathrm{E}-05$ & 7.55E-09 & $6.93 \mathrm{E}-03$ & $-4.40 \mathrm{E}-04$ & 7.37E-03 & 7.98E-07 & $2.50 \mathrm{E}-01$ \\
\hline 100 & 33.0 & $6.63 \mathrm{E}-05$ & 8.42E-09 & 7.01E-03 & $-4.40 \mathrm{E}-04$ & $7.45 \mathrm{E}-03$ & $8.90 \mathrm{E}-07$ & $2.46 \mathrm{E}-01$ \\
\hline 100 & 32.0 & $6.71 \mathrm{E}-05$ & $8.51 \mathrm{E}-09$ & 7.09E-03 & $-4.40 \mathrm{E}-04$ & $7.53 \mathrm{E}-03$ & 8.99E-07 & $2.41 \mathrm{E}-01$ \\
\hline 100 & 31.0 & $6.81 \mathrm{E}-05$ & $5.85 \mathrm{E}-09$ & $7.20 \mathrm{E}-03$ & $-4.40 \mathrm{E}-04$ & 7.64E-03 & $6.18 \mathrm{E}-07$ & $2.37 \mathrm{E}-01$ \\
\hline 100 & 30.0 & $6.92 \mathrm{E}-05$ & 5.41E-09 & 7.31E-03 & $-4.40 \mathrm{E}-04$ & $7.75 \mathrm{E}-03$ & $5.72 \mathrm{E}-07$ & $2.33 \mathrm{E}-01$ \\
\hline 100 & 29.0 & 7.04E-05 & 3.93E-09 & $7.45 \mathrm{E}-03$ & $-4.40 \mathrm{E}-04$ & 7.89E-03 & $4.16 \mathrm{E}-07$ & $2.29 \mathrm{E}-01$ \\
\hline 100 & 28.0 & 7.19E-05 & $5.56 \mathrm{E}-09$ & $7.60 \mathrm{E}-03$ & $-4.40 \mathrm{E}-04$ & $8.04 \mathrm{E}-03$ & $5.88 \mathrm{E}-07$ & $2.25 \mathrm{E}-01$ \\
\hline 100 & 27.0 & $7.35 \mathrm{E}-05$ & $3.95 \mathrm{E}-09$ & 7.77E-03 & $-4.40 \mathrm{E}-04$ & $8.21 \mathrm{E}-03$ & 4.18E-07 & $2.22 \mathrm{E}-01$ \\
\hline 100 & 26.0 & 7.54E-05 & 7.72E-09 & 7.97E-03 & $-4.40 \mathrm{E}-04$ & $8.41 \mathrm{E}-03$ & $8.16 \mathrm{E}-07$ & $2.18 \mathrm{E}-01$ \\
\hline 100 & 25.0 & 7.74E-05 & $9.00 \mathrm{E}-09$ & $8.18 \mathrm{E}-03$ & $-4.40 \mathrm{E}-04$ & $8.62 \mathrm{E}-03$ & $9.51 \mathrm{E}-07$ & $2.16 \mathrm{E}-01$ \\
\hline 100 & 24.0 & 7.98E-05 & 4.97E-09 & $8.43 \mathrm{E}-03$ & $-4.40 \mathrm{E}-04$ & 8.87E-03 & $5.25 \mathrm{E}-07$ & $2.13 \mathrm{E}-01$ \\
\hline 100 & 23.0 & 8.23E-05 & 5.30E-08 & 8.70E-03 & $-4.40 \mathrm{E}-04$ & $9.14 \mathrm{E}-03$ & $5.61 \mathrm{E}-06$ & $2.10 \mathrm{E}-01$ \\
\hline 100 & 22.0 & $8.52 \mathrm{E}-05$ & $2.48 \mathrm{E}-08$ & $9.00 \mathrm{E}-03$ & $-4.40 \mathrm{E}-04$ & $9.44 \mathrm{E}-03$ & $2.62 \mathrm{E}-06$ & $2.08 \mathrm{E}-01$ \\
\hline 100 & 21.0 & $8.85 \mathrm{E}-05$ & $3.63 \mathrm{E}-08$ & $9.36 \mathrm{E}-03$ & $-4.40 \mathrm{E}-04$ & $9.80 \mathrm{E}-03$ & $3.84 \mathrm{E}-06$ & $2.06 \mathrm{E}-01$ \\
\hline 100 & 20.0 & $9.22 \mathrm{E}-05$ & 2.44E-08 & $9.75 \mathrm{E}-03$ & $-4.40 \mathrm{E}-04$ & $1.02 \mathrm{E}-02$ & $2.58 \mathrm{E}-06$ & $2.04 \mathrm{E}-01$ \\
\hline 100 & 19.0 & $9.64 \mathrm{E}-05$ & $3.12 \mathrm{E}-08$ & $1.02 \mathrm{E}-02$ & $-4.40 \mathrm{E}-04$ & $1.06 \mathrm{E}-02$ & $3.30 \mathrm{E}-06$ & $2.02 \mathrm{E}-01$ \\
\hline 100 & 18.0 & $1.01 \mathrm{E}-04$ & $1.88 \mathrm{E}-08$ & $1.07 \mathrm{E}-02$ & $-4.40 \mathrm{E}-04$ & $1.11 \mathrm{E}-02$ & $1.99 \mathrm{E}-06$ & $2.00 \mathrm{E}-01$ \\
\hline 100 & 17.0 & $1.06 \mathrm{E}-04$ & $2.50 \mathrm{E}-09$ & $1.12 \mathrm{E}-02$ & $-4.40 \mathrm{E}-04$ & $1.17 \mathrm{E}-02$ & 2.64E-07 & $1.99 \mathrm{E}-01$ \\
\hline 100 & 16.0 & $1.12 \mathrm{E}-04$ & $2.42 \mathrm{E}-08$ & 1.19E-02 & $-4.40 \mathrm{E}-04$ & $1.23 \mathrm{E}-02$ & $2.56 \mathrm{E}-06$ & $1.97 \mathrm{E}-01$ \\
\hline
\end{tabular}




$\begin{array}{lllllllll}100 & 15.0 & 1.19 \mathrm{E}-04 & 6.40 \mathrm{E}-08 & 1.26 \mathrm{E}-02 & -4.40 \mathrm{E}-04 & 1.30 \mathrm{E}-02 & 6.77 \mathrm{E}-06 & 1.95 \mathrm{E}-01 \\ 100 & 14.0 & 1.27 \mathrm{E}-04 & 2.40 \mathrm{E}-08 & 1.34 \mathrm{E}-02 & -4.40 \mathrm{E}-04 & 1.39 \mathrm{E}-02 & 2.54 \mathrm{E}-06 & 1.94 \mathrm{E}-01 \\ 100 & 13.0 & 1.36 \mathrm{E}-04 & 6.96 \mathrm{E}-08 & 1.43 \mathrm{E}-02 & -4.40 \mathrm{E}-04 & 1.48 \mathrm{E}-02 & 7.36 \mathrm{E}-06 & 1.92 \mathrm{E}-01 \\ 100 & 12.0 & 1.46 \mathrm{E}-04 & 5.88 \mathrm{E}-08 & 1.54 \mathrm{E}-02 & -4.40 \mathrm{E}-04 & 1.59 \mathrm{E}-02 & 6.21 \mathrm{E}-06 & 1.90 \mathrm{E}-01 \\ 100 & 11.0 & 1.58 \mathrm{E}-04 & 9.83 \mathrm{E}-08 & 1.67 \mathrm{E}-02 & -4.40 \mathrm{E}-04 & 1.72 \mathrm{E}-02 & 1.04 \mathrm{E}-05 & 1.89 \mathrm{E}-01 \\ 100 & 10.0 & 1.73 \mathrm{E}-04 & 3.44 \mathrm{E}-08 & 1.83 \mathrm{E}-02 & -4.40 \mathrm{E}-04 & 1.87 \mathrm{E}-02 & 3.64 \mathrm{E}-06 & 1.87 \mathrm{E}-01 \\ 100 & 9.0 & 1.90 \mathrm{E}-04 & 8.16 \mathrm{E}-08 & 2.01 \mathrm{E}-02 & -4.40 \mathrm{E}-04 & 2.06 \mathrm{E}-02 & 8.63 \mathrm{E}-06 & 1.85 \mathrm{E}-01 \\ 100 & 8.0 & 2.12 \mathrm{E}-04 & 6.53 \mathrm{E}-08 & 2.24 \mathrm{E}-02 & -4.40 \mathrm{E}-04 & 2.29 \mathrm{E}-02 & 6.90 \mathrm{E}-06 & 1.83 \mathrm{E}-01 \\ 100 & 7.0 & 2.40 \mathrm{E}-04 & 1.04 \mathrm{E}-07 & 2.54 \mathrm{E}-02 & -4.40 \mathrm{E}-04 & 2.58 \mathrm{E}-02 & 1.10 \mathrm{E}-05 & 1.81 \mathrm{E}-01 \\ 100 & 6.0 & 2.76 \mathrm{E}-04 & 1.40 \mathrm{E}-07 & 2.92 \mathrm{E}-02 & -4.40 \mathrm{E}-04 & 2.97 \mathrm{E}-02 & 1.48 \mathrm{E}-05 & 1.78 \mathrm{E}-01 \\ 100 & 5.0 & 3.27 \mathrm{E}-04 & 2.71 \mathrm{E}-07 & 3.45 \mathrm{E}-02 & -4.40 \mathrm{E}-04 & 3.50 \mathrm{E}-02 & 2.87 \mathrm{E}-05 & 1.75 \mathrm{E}-01\end{array}$


Complex 6 ([\{ $\left.\mathrm{Mn}\left(\mathrm{CH}_{2} \mathrm{CMe}_{3}\right)\left(\mu-\mathrm{CH}_{2} \mathrm{CMe}_{3}\right)\right\}_{2}(\mu$-dmpe $\left.)\right]$

Mass (mg): 62.5

Field

\begin{tabular}{|c|c|c|c|c|c|c|c|c|}
\hline $\begin{array}{l}\text { Field } \\
\text { (Oe) }\end{array}$ & Temp (K) & EMU & STD & $\begin{array}{c}\text { Chi } \\
\text { (cm3/mol) }\end{array}$ & $\begin{array}{c}\mathrm{dia} \\
(\mathrm{cm} 3 / \mathrm{mol})\end{array}$ & $\begin{array}{l}\text { Cor Chi } \\
(\mathrm{cm} 3 / \mathrm{mol})\end{array}$ & esd & $\begin{array}{c}\text { Chi*T } \\
(\mathrm{cm} 3 \mathrm{~K} / \mathrm{mol}\end{array}$ \\
\hline 100 & 300.0 & 7.11E-05 & $3.15 \mathrm{E}-08$ & $6.19 \mathrm{E}-03$ & $-4.12 \mathrm{E}-04$ & $6.61 \mathrm{E}-03$ & $-5.27 \mathrm{E}-05$ & $1.98 \mathrm{E}+00$ \\
\hline 100 & 294.7 & 7.07E-05 & 3.22E-08 & $6.16 \mathrm{E}-03$ & $-4.12 \mathrm{E}-04$ & $6.58 \mathrm{E}-03$ & $-5.25 \mathrm{E}-05$ & $1.94 \mathrm{E}+00$ \\
\hline 100 & 289.7 & $7.05 \mathrm{E}-05$ & $1.35 \mathrm{E}-08$ & $6.15 \mathrm{E}-03$ & $-4.12 \mathrm{E}-04$ & $6.56 \mathrm{E}-03$ & $-5.07 \mathrm{E}-05$ & $1.90 \mathrm{E}+00$ \\
\hline 100 & 284.7 & 7.03E-05 & $2.10 \mathrm{E}-08$ & $6.13 \mathrm{E}-03$ & $-4.12 \mathrm{E}-04$ & $6.54 \mathrm{E}-03$ & $-5.13 \mathrm{E}-05$ & $1.86 \mathrm{E}+00$ \\
\hline 100 & 279.7 & 7.01E-05 & $1.78 \mathrm{E}-08$ & $6.11 \mathrm{E}-03$ & $-4.12 \mathrm{E}-04$ & $6.52 \mathrm{E}-03$ & $-5.08 \mathrm{E}-05$ & $1.82 \mathrm{E}+00$ \\
\hline 100 & 274.7 & $6.99 \mathrm{E}-05$ & $1.90 \mathrm{E}-08$ & $6.09 \mathrm{E}-03$ & $-4.12 \mathrm{E}-04$ & $6.50 \mathrm{E}-03$ & $-5.08 \mathrm{E}-05$ & $1.79 \mathrm{E}+00$ \\
\hline 100 & 269.7 & $6.97 \mathrm{E}-05$ & $1.19 \mathrm{E}-08$ & $6.07 \mathrm{E}-03$ & $-4.12 \mathrm{E}-04$ & $6.48 \mathrm{E}-03$ & $-5.00 \mathrm{E}-05$ & $1.75 \mathrm{E}+00$ \\
\hline 100 & 264.7 & $6.94 \mathrm{E}-05$ & $1.45 \mathrm{E}-08$ & $6.05 \mathrm{E}-03$ & $-4.12 \mathrm{E}-04$ & $6.46 \mathrm{E}-03$ & $-5.01 \mathrm{E}-05$ & $1.71 \mathrm{E}+00$ \\
\hline 100 & 259.7 & $6.92 \mathrm{E}-05$ & $2.06 \mathrm{E}-08$ & $6.03 \mathrm{E}-03$ & $-4.12 \mathrm{E}-04$ & $6.44 \mathrm{E}-03$ & $-5.04 \mathrm{E}-05$ & $1.67 \mathrm{E}+00$ \\
\hline 100 & 254.7 & $6.91 \mathrm{E}-05$ & $1.58 \mathrm{E}-08$ & $6.02 \mathrm{E}-03$ & $-4.12 \mathrm{E}-04$ & $6.43 \mathrm{E}-03$ & $-4.99 \mathrm{E}-05$ & $1.64 \mathrm{E}+00$ \\
\hline 100 & 249.7 & $6.90 \mathrm{E}-05$ & 8.37E-09 & $6.01 \mathrm{E}-03$ & $-4.12 \mathrm{E}-04$ & $6.43 \mathrm{E}-03$ & $-4.92 \mathrm{E}-05$ & $1.60 \mathrm{E}+00$ \\
\hline 100 & 244.7 & $6.89 \mathrm{E}-05$ & 4.05E-09 & $6.01 \mathrm{E}-03$ & $-4.12 \mathrm{E}-04$ & $6.42 \mathrm{E}-03$ & $-4.88 \mathrm{E}-05$ & $1.57 \mathrm{E}+00$ \\
\hline 100 & 239.7 & $6.88 \mathrm{E}-05$ & $1.27 \mathrm{E}-08$ & $6.00 \mathrm{E}-03$ & $-4.12 \mathrm{E}-04$ & $6.41 \mathrm{E}-03$ & $-4.95 E-05$ & $1.54 \mathrm{E}+00$ \\
\hline 100 & 234.7 & $6.87 \mathrm{E}-05$ & $1.18 \mathrm{E}-08$ & $5.99 \mathrm{E}-03$ & $-4.12 \mathrm{E}-04$ & $6.40 \mathrm{E}-03$ & $-4.93 \mathrm{E}-05$ & $1.50 \mathrm{E}+00$ \\
\hline 100 & 229.7 & $6.86 \mathrm{E}-05$ & $1.37 \mathrm{E}-08$ & $5.98 \mathrm{E}-03$ & $-4.12 \mathrm{E}-04$ & $6.39 \mathrm{E}-03$ & $-4.94 \mathrm{E}-05$ & $1.47 \mathrm{E}+00$ \\
\hline 100 & 224.7 & $6.85 \mathrm{E}-05$ & $1.04 \mathrm{E}-08$ & $5.97 \mathrm{E}-03$ & $-4.12 \mathrm{E}-04$ & $6.38 \mathrm{E}-03$ & $-4.90 \mathrm{E}-05$ & $1.43 \mathrm{E}+00$ \\
\hline 100 & 219.7 & $6.83 \mathrm{E}-05$ & $1.23 \mathrm{E}-08$ & $5.95 \mathrm{E}-03$ & $-4.12 \mathrm{E}-04$ & $6.36 \mathrm{E}-03$ & $-4.91 \mathrm{E}-05$ & $1.40 \mathrm{E}+00$ \\
\hline 100 & 214.7 & $6.81 \mathrm{E}-05$ & $1.23 \mathrm{E}-08$ & $5.94 \mathrm{E}-03$ & $-4.12 \mathrm{E}-04$ & $6.35 \mathrm{E}-03$ & $-4.89 \mathrm{E}-05$ & $1.36 \mathrm{E}+00$ \\
\hline 100 & 209.7 & $6.79 \mathrm{E}-05$ & 4.12E-09 & $5.92 \mathrm{E}-03$ & $-4.12 \mathrm{E}-04$ & $6.33 \mathrm{E}-03$ & $-4.81 \mathrm{E}-05$ & $1.33 \mathrm{E}+00$ \\
\hline 100 & 204.7 & $6.77 \mathrm{E}-05$ & $2.29 \mathrm{E}-08$ & $5.90 \mathrm{E}-03$ & $-4.12 \mathrm{E}-04$ & $6.31 \mathrm{E}-03$ & $-4.96 \mathrm{E}-05$ & $1.29 \mathrm{E}+00$ \\
\hline 100 & 199.7 & $6.74 \mathrm{E}-05$ & $1.86 \mathrm{E}-08$ & $5.87 \mathrm{E}-03$ & $-4.12 \mathrm{E}-04$ & $6.28 \mathrm{E}-03$ & $-4.90 \mathrm{E}-05$ & $1.26 \mathrm{E}+00$ \\
\hline 100 & 194.7 & $6.72 \mathrm{E}-05$ & $1.80 \mathrm{E}-08$ & $5.85 \mathrm{E}-03$ & $-4.12 \mathrm{E}-04$ & $6.26 \mathrm{E}-03$ & $-4.88 \mathrm{E}-05$ & $1.22 \mathrm{E}+00$ \\
\hline 100 & 189.8 & $6.69 \mathrm{E}-05$ & $2.25 \mathrm{E}-08$ & $5.83 \mathrm{E}-03$ & $-4.12 \mathrm{E}-04$ & $6.24 \mathrm{E}-03$ & $-4.90 \mathrm{E}-05$ & $1.18 \mathrm{E}+00$ \\
\hline 100 & 184.8 & $6.67 \mathrm{E}-05$ & $8.02 \mathrm{E}-09$ & $5.81 \mathrm{E}-03$ & $-4.12 \mathrm{E}-04$ & $6.22 \mathrm{E}-03$ & $-4.75 \mathrm{E}-05$ & $1.15 \mathrm{E}+00$ \\
\hline 100 & 179.8 & $6.64 \mathrm{E}-05$ & $1.84 \mathrm{E}-08$ & $5.78 \mathrm{E}-03$ & $-4.12 \mathrm{E}-04$ & $6.19 \mathrm{E}-03$ & $-4.82 \mathrm{E}-05$ & $1.11 \mathrm{E}+00$ \\
\hline 100 & 174.8 & $6.60 \mathrm{E}-05$ & $6.96 \mathrm{E}-09$ & $5.76 \mathrm{E}-03$ & $-4.12 \mathrm{E}-04$ & $6.17 \mathrm{E}-03$ & $-4.70 \mathrm{E}-05$ & $1.08 \mathrm{E}+00$ \\
\hline 100 & 169.8 & $6.57 \mathrm{E}-05$ & $2.49 \mathrm{E}-08$ & $5.72 \mathrm{E}-03$ & $-4.12 \mathrm{E}-04$ & $6.14 \mathrm{E}-03$ & $-4.84 \mathrm{E}-05$ & $1.04 \mathrm{E}+00$ \\
\hline 100 & 164.8 & $6.54 \mathrm{E}-05$ & $1.45 \mathrm{E}-08$ & $5.69 \mathrm{E}-03$ & $-4.12 \mathrm{E}-04$ & $6.11 \mathrm{E}-03$ & $-4.72 \mathrm{E}-05$ & $1.01 \mathrm{E}+00$ \\
\hline 100 & 159.8 & $6.51 \mathrm{E}-05$ & $9.13 \mathrm{E}-09$ & $5.67 \mathrm{E}-03$ & $-4.12 \mathrm{E}-04$ & $6.08 \mathrm{E}-03$ & $-4.65 \mathrm{E}-05$ & $9.72 \mathrm{E}-01$ \\
\hline 100 & 154.8 & $6.54 \mathrm{E}-05$ & $7.53 \mathrm{E}-08$ & $5.70 \mathrm{E}-03$ & $-4.12 \mathrm{E}-04$ & $6.11 \mathrm{E}-03$ & $-5.26 \mathrm{E}-05$ & $9.46 \mathrm{E}-01$ \\
\hline 100 & 149.9 & $6.62 \mathrm{E}-05$ & $1.05 \mathrm{E}-08$ & $5.77 \mathrm{E}-03$ & $-4.12 \mathrm{E}-04$ & $6.18 \mathrm{E}-03$ & $-4.74 \mathrm{E}-05$ & $9.27 \mathrm{E}-01$ \\
\hline 100 & 144.9 & $6.62 \mathrm{E}-05$ & $2.16 \mathrm{E}-09$ & $5.76 \mathrm{E}-03$ & $-4.12 \mathrm{E}-04$ & $6.18 \mathrm{E}-03$ & $-4.67 \mathrm{E}-05$ & 8.95E-01 \\
\hline 100 & 139.9 & $6.59 \mathrm{E}-05$ & $6.35 \mathrm{E}-09$ & $5.74 \mathrm{E}-03$ & $-4.12 \mathrm{E}-04$ & $6.16 \mathrm{E}-03$ & $-4.69 \mathrm{E}-05$ & 8.61E-01 \\
\hline 100 & 134.9 & $6.56 \mathrm{E}-05$ & $1.09 \mathrm{E}-08$ & $5.72 \mathrm{E}-03$ & $-4.12 \mathrm{E}-04$ & $6.13 \mathrm{E}-03$ & $-4.71 \mathrm{E}-05$ & $8.27 \mathrm{E}-01$ \\
\hline 100 & 129.9 & $6.52 \mathrm{E}-05$ & $1.42 \mathrm{E}-08$ & $5.69 \mathrm{E}-03$ & $-4.12 \mathrm{E}-04$ & $6.10 \mathrm{E}-03$ & $-4.71 \mathrm{E}-05$ & 7.92E-01 \\
\hline 100 & 124.9 & $6.50 \mathrm{E}-05$ & $3.74 \mathrm{E}-08$ & $5.66 \mathrm{E}-03$ & $-4.12 \mathrm{E}-04$ & $6.08 \mathrm{E}-03$ & $-4.90 \mathrm{E}-05$ & 7.59E-01 \\
\hline 100 & 120.0 & $6.46 \mathrm{E}-05$ & $3.89 \mathrm{E}-08$ & $5.63 \mathrm{E}-03$ & $-4.12 \mathrm{E}-04$ & $6.04 \mathrm{E}-03$ & $-4.88 \mathrm{E}-05$ & $7.25 \mathrm{E}-01$ \\
\hline 100 & 115.0 & $6.42 \mathrm{E}-05$ & 2.19E-08 & $5.59 \mathrm{E}-03$ & $-4.12 \mathrm{E}-04$ & $6.00 \mathrm{E}-03$ & $-4.70 \mathrm{E}-05$ & $6.90 \mathrm{E}-01$ \\
\hline 100 & 110.0 & $6.36 \mathrm{E}-05$ & $2.73 \mathrm{E}-08$ & $5.54 \mathrm{E}-03$ & $-4.12 \mathrm{E}-04$ & $5.95 \mathrm{E}-03$ & $-4.71 \mathrm{E}-05$ & $6.55 \mathrm{E}-01$ \\
\hline
\end{tabular}




\begin{tabular}{|c|c|c|c|c|c|c|c|c|}
\hline 100 & 105.0 & $6.30 \mathrm{E}-05$ & $3.12 \mathrm{E}-08$ & $5.49 \mathrm{E}-03$ & $-4.12 \mathrm{E}-04$ & $5.90 \mathrm{E}-03$ & $-4.70 \mathrm{E}-05$ & $6.19 \mathrm{E}-01$ \\
\hline 100 & 100.0 & $6.23 \mathrm{E}-05$ & $2.41 \mathrm{E}-08$ & $5.43 \mathrm{E}-03$ & $-4.12 \mathrm{E}-04$ & $5.84 \mathrm{E}-03$ & $-4.59 \mathrm{E}-05$ & $5.84 \mathrm{E}-01$ \\
\hline 100 & 98.9 & $6.22 \mathrm{E}-05$ & $1.12 \mathrm{E}-08$ & $5.42 \mathrm{E}-03$ & $-4.12 \mathrm{E}-04$ & $5.83 \mathrm{E}-03$ & $-4.47 \mathrm{E}-05$ & $5.77 \mathrm{E}-01$ \\
\hline 100 & 97.9 & $6.20 \mathrm{E}-05$ & $2.30 \mathrm{E}-08$ & $5.40 \mathrm{E}-03$ & $-4.12 \mathrm{E}-04$ & $5.82 \mathrm{E}-03$ & $-4.56 \mathrm{E}-05$ & $5.69 \mathrm{E}-01$ \\
\hline 100 & 96.9 & $6.19 \mathrm{E}-05$ & $1.62 \mathrm{E}-08$ & $5.39 \mathrm{E}-03$ & $-4.12 \mathrm{E}-04$ & $5.80 \mathrm{E}-03$ & $-4.49 \mathrm{E}-05$ & 5.62E-01 \\
\hline 100 & 95.9 & $6.17 \mathrm{E}-05$ & $4.31 \mathrm{E}-08$ & $5.38 \mathrm{E}-03$ & $-4.12 \mathrm{E}-04$ & $5.79 \mathrm{E}-03$ & $-4.72 \mathrm{E}-05$ & $5.56 \mathrm{E}-01$ \\
\hline 100 & 94.9 & $6.16 \mathrm{E}-05$ & $2.59 \mathrm{E}-08$ & $5.37 \mathrm{E}-03$ & $-4.12 \mathrm{E}-04$ & $5.78 \mathrm{E}-03$ & $-4.56 \mathrm{E}-05$ & $5.49 \mathrm{E}-01$ \\
\hline 100 & 93.9 & $6.14 \mathrm{E}-05$ & $1.19 \mathrm{E}-08$ & $5.35 \mathrm{E}-03$ & $-4.12 \mathrm{E}-04$ & $5.77 \mathrm{E}-03$ & $-4.42 \mathrm{E}-05$ & $5.41 \mathrm{E}-01$ \\
\hline 100 & 92.9 & $6.12 \mathrm{E}-05$ & $2.57 \mathrm{E}-08$ & $5.34 \mathrm{E}-03$ & $-4.12 \mathrm{E}-04$ & $5.75 \mathrm{E}-03$ & $-4.53 \mathrm{E}-05$ & 5.34E-01 \\
\hline 100 & 91.9 & $6.11 \mathrm{E}-05$ & $1.29 \mathrm{E}-08$ & $5.32 \mathrm{E}-03$ & $-4.12 \mathrm{E}-04$ & $5.73 \mathrm{E}-03$ & $-4.40 \mathrm{E}-05$ & $5.27 \mathrm{E}-01$ \\
\hline 100 & 90.9 & $6.09 \mathrm{E}-05$ & 3.61E-08 & $5.30 \mathrm{E}-03$ & $-4.12 \mathrm{E}-04$ & $5.72 \mathrm{E}-03$ & $-4.59 \mathrm{E}-05$ & $5.20 \mathrm{E}-01$ \\
\hline 100 & 89.9 & $6.07 \mathrm{E}-05$ & $2.08 \mathrm{E}-08$ & $5.29 \mathrm{E}-03$ & $-4.12 \mathrm{E}-04$ & $5.70 \mathrm{E}-03$ & $-4.45 \mathrm{E}-05$ & $5.13 \mathrm{E}-01$ \\
\hline 100 & 88.9 & $6.05 \mathrm{E}-05$ & $2.23 \mathrm{E}-08$ & $5.27 \mathrm{E}-03$ & $-4.12 \mathrm{E}-04$ & $5.68 \mathrm{E}-03$ & $-4.45 \mathrm{E}-05$ & $5.05 \mathrm{E}-01$ \\
\hline 100 & 87.9 & $6.03 \mathrm{E}-05$ & $2.14 \mathrm{E}-08$ & $5.25 \mathrm{E}-03$ & $-4.12 \mathrm{E}-04$ & $5.67 \mathrm{E}-03$ & $-4.43 \mathrm{E}-05$ & $4.98 \mathrm{E}-01$ \\
\hline 100 & 86.9 & $6.04 \mathrm{E}-05$ & 2.43E-08 & $5.26 \mathrm{E}-03$ & $-4.12 \mathrm{E}-04$ & $5.67 \mathrm{E}-03$ & $-4.46 \mathrm{E}-05$ & 4.93E-01 \\
\hline 100 & 85.9 & $6.02 \mathrm{E}-05$ & 2.30E-08 & $5.24 \mathrm{E}-03$ & $-4.12 \mathrm{E}-04$ & $5.65 \mathrm{E}-03$ & $-4.43 \mathrm{E}-05$ & $4.86 \mathrm{E}-01$ \\
\hline 100 & 84.9 & 5.99E-05 & 3.34E-08 & $5.22 \mathrm{E}-03$ & $-4.12 \mathrm{E}-04$ & $5.64 \mathrm{E}-03$ & $-4.51 \mathrm{E}-05$ & $4.79 \mathrm{E}-01$ \\
\hline 100 & 83.9 & $5.97 \mathrm{E}-05$ & $2.56 \mathrm{E}-08$ & $5.21 \mathrm{E}-03$ & $-4.12 \mathrm{E}-04$ & $5.62 \mathrm{E}-03$ & $-4.42 \mathrm{E}-05$ & 4.71E-01 \\
\hline 100 & 82.9 & $5.95 \mathrm{E}-05$ & 2.22E-08 & $5.18 \mathrm{E}-03$ & $-4.12 \mathrm{E}-04$ & $5.60 \mathrm{E}-03$ & $-4.38 \mathrm{E}-05$ & 4.64E-01 \\
\hline 100 & 81.9 & $5.93 \mathrm{E}-05$ & $3.21 \mathrm{E}-08$ & $5.16 \mathrm{E}-03$ & $-4.12 \mathrm{E}-04$ & $5.58 \mathrm{E}-03$ & $-4.45 \mathrm{E}-05$ & 4.57E-01 \\
\hline 100 & 80.9 & $5.90 \mathrm{E}-05$ & $2.80 \mathrm{E}-08$ & $5.14 \mathrm{E}-03$ & $-4.12 \mathrm{E}-04$ & $5.56 \mathrm{E}-03$ & $-4.39 \mathrm{E}-05$ & $4.50 \mathrm{E}-01$ \\
\hline 100 & 80.0 & $5.88 \mathrm{E}-05$ & $3.62 \mathrm{E}-08$ & $5.12 \mathrm{E}-03$ & $-4.12 \mathrm{E}-04$ & $5.53 \mathrm{E}-03$ & $-4.45 \mathrm{E}-05$ & 4.42E-01 \\
\hline 100 & 79.0 & $5.85 \mathrm{E}-05$ & 4.43E-08 & $5.10 \mathrm{E}-03$ & $-4.12 \mathrm{E}-04$ & $5.51 \mathrm{E}-03$ & $-4.50 \mathrm{E}-05$ & 4.35E-01 \\
\hline 100 & 78.0 & 5.83E-05 & 3.99E-08 & $5.08 \mathrm{E}-03$ & $-4.12 \mathrm{E}-04$ & $5.49 \mathrm{E}-03$ & $-4.44 \mathrm{E}-05$ & 4.28E-01 \\
\hline 100 & 77.0 & $5.80 \mathrm{E}-05$ & $3.26 \mathrm{E}-08$ & $5.05 \mathrm{E}-03$ & $-4.12 \mathrm{E}-04$ & $5.46 \mathrm{E}-03$ & $-4.36 \mathrm{E}-05$ & 4.21E-01 \\
\hline 100 & 76.0 & 5.77E-05 & $2.98 \mathrm{E}-08$ & $5.03 \mathrm{E}-03$ & $-4.12 \mathrm{E}-04$ & $5.44 \mathrm{E}-03$ & $-4.31 \mathrm{E}-05$ & 4.13E-01 \\
\hline 100 & 75.0 & $5.74 \mathrm{E}-05$ & 4.46E-08 & $5.00 \mathrm{E}-03$ & $-4.12 \mathrm{E}-04$ & $5.41 \mathrm{E}-03$ & $-4.42 \mathrm{E}-05$ & 4.06E-01 \\
\hline 100 & 74.0 & 5.71E-05 & $4.52 \mathrm{E}-08$ & $4.97 \mathrm{E}-03$ & $-4.12 \mathrm{E}-04$ & $5.39 \mathrm{E}-03$ & $-4.41 \mathrm{E}-05$ & $3.99 \mathrm{E}-01$ \\
\hline 100 & 73.0 & $5.68 \mathrm{E}-05$ & 4.59E-08 & $4.95 \mathrm{E}-03$ & $-4.12 \mathrm{E}-04$ & $5.36 \mathrm{E}-03$ & $-4.39 \mathrm{E}-05$ & $3.91 \mathrm{E}-01$ \\
\hline 100 & 72.0 & $5.61 \mathrm{E}-05$ & $2.56 \mathrm{E}-07$ & $4.89 \mathrm{E}-03$ & $-4.12 \mathrm{E}-04$ & $5.30 \mathrm{E}-03$ & $-6.19 \mathrm{E}-05$ & $3.81 \mathrm{E}-01$ \\
\hline 100 & 71.0 & $5.58 \mathrm{E}-05$ & 2.99E-08 & 4.87E-03 & $-4.12 \mathrm{E}-04$ & $5.28 \mathrm{E}-03$ & $-4.19 \mathrm{E}-05$ & $3.75 \mathrm{E}-01$ \\
\hline 100 & 70.0 & 5.55E-05 & 4.46E-08 & $4.83 \mathrm{E}-03$ & $-4.12 \mathrm{E}-04$ & $5.25 \mathrm{E}-03$ & $-4.29 \mathrm{E}-05$ & $3.67 \mathrm{E}-01$ \\
\hline 100 & 69.0 & $5.51 \mathrm{E}-05$ & 4.94E-08 & $4.80 \mathrm{E}-03$ & $-4.12 \mathrm{E}-04$ & $5.22 \mathrm{E}-03$ & $-4.31 \mathrm{E}-05$ & $3.60 \mathrm{E}-01$ \\
\hline 100 & 68.0 & $5.48 \mathrm{E}-05$ & $5.27 \mathrm{E}-08$ & $4.77 \mathrm{E}-03$ & $-4.12 \mathrm{E}-04$ & $5.18 \mathrm{E}-03$ & $-4.31 \mathrm{E}-05$ & $3.53 \mathrm{E}-01$ \\
\hline 100 & 67.0 & $5.44 \mathrm{E}-05$ & 4.23E-08 & 4.74E-03 & $-4.12 \mathrm{E}-04$ & $5.15 \mathrm{E}-03$ & $-4.19 \mathrm{E}-05$ & $3.45 \mathrm{E}-01$ \\
\hline 100 & 66.0 & $5.40 \mathrm{E}-05$ & $4.23 \mathrm{E}-08$ & $4.71 \mathrm{E}-03$ & $-4.12 \mathrm{E}-04$ & $5.12 \mathrm{E}-03$ & $-4.17 \mathrm{E}-05$ & $3.38 \mathrm{E}-01$ \\
\hline 100 & 65.0 & 5.37E-05 & $1.09 \mathrm{E}-08$ & $4.68 \mathrm{E}-03$ & $-4.12 \mathrm{E}-04$ & $5.09 \mathrm{E}-03$ & $-3.87 \mathrm{E}-05$ & 3.31E-01 \\
\hline 100 & 64.0 & $5.33 \mathrm{E}-05$ & 5.93E-09 & 4.64E-03 & $-4.12 \mathrm{E}-04$ & $5.05 \mathrm{E}-03$ & $-3.79 \mathrm{E}-05$ & $3.24 \mathrm{E}-01$ \\
\hline 100 & 63.0 & $5.28 \mathrm{E}-05$ & 7.59E-09 & $4.60 \mathrm{E}-03$ & $-4.12 \mathrm{E}-04$ & $5.01 \mathrm{E}-03$ & $-3.78 \mathrm{E}-05$ & $3.16 \mathrm{E}-01$ \\
\hline 100 & 62.0 & $5.24 \mathrm{E}-05$ & $3.13 \mathrm{E}-09$ & $4.56 \mathrm{E}-03$ & $-4.12 \mathrm{E}-04$ & 4.97E-03 & $-3.71 \mathrm{E}-05$ & $3.09 \mathrm{E}-01$ \\
\hline 100 & 61.0 & $5.21 \mathrm{E}-05$ & $1.30 \mathrm{E}-07$ & $4.54 \mathrm{E}-03$ & $-4.12 \mathrm{E}-04$ & $4.95 \mathrm{E}-03$ & $-4.80 \mathrm{E}-05$ & $3.02 \mathrm{E}-01$ \\
\hline 100 & 60.0 & $5.16 \mathrm{E}-05$ & $7.21 \mathrm{E}-08$ & $4.50 \mathrm{E}-03$ & $-4.12 \mathrm{E}-04$ & 4.91E-03 & $-4.26 \mathrm{E}-05$ & $2.95 \mathrm{E}-01$ \\
\hline 100 & 59.0 & $5.11 \mathrm{E}-05$ & 8.11E-09 & $4.45 \mathrm{E}-03$ & $-4.12 \mathrm{E}-04$ & 4.86E-03 & $-3.66 \mathrm{E}-05$ & $2.87 \mathrm{E}-01$ \\
\hline
\end{tabular}




\begin{tabular}{|c|c|c|c|c|c|c|c|c|}
\hline 100 & 58.0 & $5.06 \mathrm{E}-05$ & $2.60 \mathrm{E}-08$ & 4.41E-03 & $-4.12 \mathrm{E}-04$ & 4.82E-03 & $-3.78 \mathrm{E}-05$ & $2.80 \mathrm{E}-01$ \\
\hline 100 & 57.0 & $5.00 \mathrm{E}-05$ & $7.75 \mathrm{E}-08$ & $4.35 \mathrm{E}-03$ & $-4.12 \mathrm{E}-04$ & 4.77E-03 & $-4.19 \mathrm{E}-05$ & 2.72E-01 \\
\hline 100 & 56.0 & 4.94E-05 & $1.73 \mathrm{E}-08$ & $4.30 \mathrm{E}-03$ & $-4.12 \mathrm{E}-04$ & $4.72 \mathrm{E}-03$ & $-3.62 \mathrm{E}-05$ & $2.64 \mathrm{E}-01$ \\
\hline 100 & 55.0 & 4.89E-05 & $9.68 \mathrm{E}-09$ & 4.26E-03 & $-4.12 \mathrm{E}-04$ & 4.67E-03 & $-3.52 \mathrm{E}-05$ & $2.57 \mathrm{E}-01$ \\
\hline 100 & 54.0 & 4.83E-05 & $1.78 \mathrm{E}-08$ & $4.21 \mathrm{E}-03$ & $-4.12 \mathrm{E}-04$ & 4.62E-03 & $-3.55 \mathrm{E}-05$ & $2.50 \mathrm{E}-01$ \\
\hline 100 & 53.1 & $4.78 \mathrm{E}-05$ & $9.48 \mathrm{E}-09$ & $4.17 \mathrm{E}-03$ & $-4.12 \mathrm{E}-04$ & $4.58 \mathrm{E}-03$ & $-3.44 \mathrm{E}-05$ & $2.43 \mathrm{E}-01$ \\
\hline 100 & 52.0 & 4.73E-05 & 8.07E-09 & 4.12E-03 & $-4.12 \mathrm{E}-04$ & $4.53 \mathrm{E}-03$ & $-3.39 \mathrm{E}-05$ & $2.36 \mathrm{E}-01$ \\
\hline 100 & 51.0 & 4.67E-05 & 8.71E-09 & 4.07E-03 & $-4.12 \mathrm{E}-04$ & 4.48E-03 & $-3.36 \mathrm{E}-05$ & $2.29 \mathrm{E}-01$ \\
\hline 100 & 50.0 & 4.61E-05 & $1.55 \mathrm{E}-08$ & $4.02 \mathrm{E}-03$ & $-4.12 \mathrm{E}-04$ & $4.43 \mathrm{E}-03$ & $-3.38 \mathrm{E}-05$ & $2.22 \mathrm{E}-01$ \\
\hline 100 & 49.0 & $4.56 \mathrm{E}-05$ & $9.24 \mathrm{E}-09$ & $3.97 \mathrm{E}-03$ & $-4.12 \mathrm{E}-04$ & $4.38 \mathrm{E}-03$ & $-3.28 \mathrm{E}-05$ & $2.15 \mathrm{E}-01$ \\
\hline 100 & 48.0 & 4.50E-05 & 8.87E-09 & $3.92 \mathrm{E}-03$ & $-4.12 \mathrm{E}-04$ & 4.33E-03 & $-3.24 \mathrm{E}-05$ & $2.08 \mathrm{E}-01$ \\
\hline 100 & 47.0 & 4.44E-05 & 7.05E-09 & $3.87 \mathrm{E}-03$ & $-4.12 \mathrm{E}-04$ & $4.28 \mathrm{E}-03$ & $-3.18 \mathrm{E}-05$ & $2.01 \mathrm{E}-01$ \\
\hline 100 & 46.0 & $4.38 \mathrm{E}-05$ & $6.58 \mathrm{E}-09$ & $3.82 \mathrm{E}-03$ & $-4.12 \mathrm{E}-04$ & $4.23 \mathrm{E}-03$ & $-3.14 \mathrm{E}-05$ & $1.95 \mathrm{E}-01$ \\
\hline 100 & 45.0 & 4.33E-05 & 9.29E-09 & $3.77 \mathrm{E}-03$ & $-4.12 \mathrm{E}-04$ & 4.18E-03 & $-3.12 \mathrm{E}-05$ & $1.88 \mathrm{E}-01$ \\
\hline 100 & 44.0 & 4.27E-05 & 4.56E-09 & $3.72 \mathrm{E}-03$ & $-4.12 \mathrm{E}-04$ & 4.13E-03 & $-3.04 \mathrm{E}-05$ & $1.82 \mathrm{E}-01$ \\
\hline 100 & 43.0 & $4.21 \mathrm{E}-05$ & 6.82E-09 & $3.67 \mathrm{E}-03$ & $-4.12 \mathrm{E}-04$ & $4.08 \mathrm{E}-03$ & $-3.02 \mathrm{E}-05$ & $1.76 \mathrm{E}-01$ \\
\hline 100 & 42.0 & 4.16E-05 & $1.09 \mathrm{E}-08$ & $3.62 \mathrm{E}-03$ & $-4.12 \mathrm{E}-04$ & 4.04E-03 & $-3.02 \mathrm{E}-05$ & $1.70 \mathrm{E}-01$ \\
\hline 100 & 41.0 & 4.14E-05 & $7.56 \mathrm{E}-08$ & $3.61 \mathrm{E}-03$ & $-4.12 \mathrm{E}-04$ & 4.02E-03 & $-3.57 \mathrm{E}-05$ & $1.65 \mathrm{E}-01$ \\
\hline 100 & 40.0 & 4.09E-05 & $2.95 \mathrm{E}-08$ & $3.57 \mathrm{E}-03$ & $-4.12 \mathrm{E}-04$ & $3.98 \mathrm{E}-03$ & $-3.14 \mathrm{E}-05$ & $1.59 \mathrm{E}-01$ \\
\hline 100 & 39.0 & 4.05E-05 & $6.45 \mathrm{E}-08$ & $3.53 \mathrm{E}-03$ & $-4.12 \mathrm{E}-04$ & $3.94 \mathrm{E}-03$ & $-3.41 \mathrm{E}-05$ & $1.54 \mathrm{E}-01$ \\
\hline 100 & 38.0 & $4.02 \mathrm{E}-05$ & $1.98 \mathrm{E}-08$ & $3.50 \mathrm{E}-03$ & $-4.12 \mathrm{E}-04$ & $3.91 \mathrm{E}-03$ & $-3.00 \mathrm{E}-05$ & $1.49 \mathrm{E}-01$ \\
\hline 100 & 37.0 & $3.98 \mathrm{E}-05$ & $2.41 \mathrm{E}-08$ & $3.47 \mathrm{E}-03$ & $-4.12 \mathrm{E}-04$ & $3.88 \mathrm{E}-03$ & $-3.01 \mathrm{E}-05$ & $1.44 \mathrm{E}-01$ \\
\hline 100 & 36.0 & $3.95 \mathrm{E}-05$ & $6.95 \mathrm{E}-08$ & $3.44 \mathrm{E}-03$ & $-4.12 \mathrm{E}-04$ & $3.86 \mathrm{E}-03$ & $-3.39 \mathrm{E}-05$ & $1.39 \mathrm{E}-01$ \\
\hline 100 & 35.0 & $3.93 \mathrm{E}-05$ & $1.12 \mathrm{E}-08$ & $3.43 \mathrm{E}-03$ & $-4.12 \mathrm{E}-04$ & $3.84 \mathrm{E}-03$ & $-2.86 \mathrm{E}-05$ & $1.34 \mathrm{E}-01$ \\
\hline 100 & 34.0 & 3.92E-05 & $1.34 \mathrm{E}-08$ & $3.41 \mathrm{E}-03$ & $-4.12 \mathrm{E}-04$ & $3.83 \mathrm{E}-03$ & $-2.87 \mathrm{E}-05$ & $1.30 \mathrm{E}-01$ \\
\hline 100 & 33.0 & $3.90 \mathrm{E}-05$ & 3.64E-08 & $3.40 \mathrm{E}-03$ & $-4.12 \mathrm{E}-04$ & $3.81 \mathrm{E}-03$ & $-3.06 \mathrm{E}-05$ & $1.26 \mathrm{E}-01$ \\
\hline 100 & 32.0 & $3.89 \mathrm{E}-05$ & $2.98 \mathrm{E}-08$ & $3.39 \mathrm{E}-03$ & $-4.12 \mathrm{E}-04$ & $3.81 \mathrm{E}-03$ & $-3.00 \mathrm{E}-05$ & $1.22 \mathrm{E}-01$ \\
\hline 100 & 31.0 & $3.91 \mathrm{E}-05$ & $6.78 \mathrm{E}-08$ & $3.41 \mathrm{E}-03$ & $-4.12 \mathrm{E}-04$ & $3.82 \mathrm{E}-03$ & $-3.34 \mathrm{E}-05$ & $1.18 \mathrm{E}-01$ \\
\hline 100 & 30.0 & $3.94 \mathrm{E}-05$ & 8.03E-09 & $3.43 \mathrm{E}-03$ & $-4.12 \mathrm{E}-04$ & $3.84 \mathrm{E}-03$ & $-2.84 \mathrm{E}-05$ & $1.15 \mathrm{E}-01$ \\
\hline 100 & 29.0 & 3.97E-05 & $1.65 \mathrm{E}-08$ & $3.46 \mathrm{E}-03$ & $-4.12 \mathrm{E}-04$ & $3.87 \mathrm{E}-03$ & $-2.93 \mathrm{E}-05$ & $1.12 \mathrm{E}-01$ \\
\hline 100 & 28.0 & 4.01E-05 & 4.67E-08 & $3.49 \mathrm{E}-03$ & $-4.12 \mathrm{E}-04$ & $3.90 \mathrm{E}-03$ & $-3.22 \mathrm{E}-05$ & $1.09 \mathrm{E}-01$ \\
\hline 100 & 27.0 & $4.06 \mathrm{E}-05$ & $1.74 \mathrm{E}-08$ & $3.54 \mathrm{E}-03$ & $-4.12 \mathrm{E}-04$ & $3.95 \mathrm{E}-03$ & $-3.00 \mathrm{E}-05$ & $1.07 \mathrm{E}-01$ \\
\hline 100 & 26.0 & 4.13E-05 & 9.93E-09 & $3.60 \mathrm{E}-03$ & $-4.12 \mathrm{E}-04$ & 4.01E-03 & $-2.99 \mathrm{E}-05$ & $1.04 \mathrm{E}-01$ \\
\hline 100 & 25.0 & $4.22 \mathrm{E}-05$ & $1.92 \mathrm{E}-08$ & $3.68 \mathrm{E}-03$ & $-4.12 \mathrm{E}-04$ & 4.09E-03 & $-3.14 \mathrm{E}-05$ & $1.02 \mathrm{E}-01$ \\
\hline 100 & 24.0 & 4.33E-05 & $2.25 \mathrm{E}-08$ & $3.78 \mathrm{E}-03$ & $-4.12 \mathrm{E}-04$ & 4.19E-03 & $-3.24 \mathrm{E}-05$ & $1.01 \mathrm{E}-01$ \\
\hline 100 & 23.0 & 4.47E-05 & $1.83 \mathrm{E}-08$ & $3.90 \mathrm{E}-03$ & $-4.12 \mathrm{E}-04$ & $4.31 \mathrm{E}-03$ & $-3.30 \mathrm{E}-05$ & $9.91 \mathrm{E}-02$ \\
\hline 100 & 22.0 & 4.63E-05 & $2.05 \mathrm{E}-08$ & $4.03 \mathrm{E}-03$ & $-4.12 \mathrm{E}-04$ & 4.44E-03 & $-3.43 \mathrm{E}-05$ & $9.77 \mathrm{E}-02$ \\
\hline 100 & 21.0 & $4.80 \mathrm{E}-05$ & $2.16 \mathrm{E}-08$ & $4.18 \mathrm{E}-03$ & $-4.12 \mathrm{E}-04$ & $4.59 \mathrm{E}-03$ & $-3.56 \mathrm{E}-05$ & $9.65 \mathrm{E}-02$ \\
\hline 100 & 20.0 & $5.00 \mathrm{E}-05$ & $3.78 \mathrm{E}-08$ & $4.36 \mathrm{E}-03$ & $-4.12 \mathrm{E}-04$ & 4.77E-03 & $-3.84 \mathrm{E}-05$ & $9.54 \mathrm{E}-02$ \\
\hline 100 & 19.0 & $5.22 \mathrm{E}-05$ & $2.02 \mathrm{E}-08$ & $4.55 \mathrm{E}-03$ & $-4.12 \mathrm{E}-04$ & $4.96 \mathrm{E}-03$ & $-3.85 \mathrm{E}-05$ & $9.43 \mathrm{E}-02$ \\
\hline 100 & 18.0 & $5.48 \mathrm{E}-05$ & $2.08 \mathrm{E}-08$ & $4.78 \mathrm{E}-03$ & $-4.12 \mathrm{E}-04$ & $5.19 \mathrm{E}-03$ & $-4.03 \mathrm{E}-05$ & $9.34 \mathrm{E}-02$ \\
\hline 100 & 17.0 & $5.78 \mathrm{E}-05$ & $3.98 \mathrm{E}-08$ & $5.03 \mathrm{E}-03$ & $-4.12 \mathrm{E}-04$ & $5.45 \mathrm{E}-03$ & $-4.41 \mathrm{E}-05$ & $9.26 \mathrm{E}-02$ \\
\hline 100 & 16.0 & $6.12 \mathrm{E}-05$ & $2.08 \mathrm{E}-08$ & 5.33E-03 & $-4.12 \mathrm{E}-04$ & $5.74 \mathrm{E}-03$ & $-4.48 \mathrm{E}-05$ & $9.20 \mathrm{E}-02$ \\
\hline
\end{tabular}




$\begin{array}{lllllllll}100 & 15.0 & 6.51 \mathrm{E}-05 & 3.22 \mathrm{E}-08 & 5.67 \mathrm{E}-03 & -4.12 \mathrm{E}-04 & 6.08 \mathrm{E}-03 & -4.86 \mathrm{E}-05 & 9.13 \mathrm{E}-02 \\ 100 & 14.0 & 6.96 \mathrm{E}-05 & 2.04 \mathrm{E}-08 & 6.06 \mathrm{E}-03 & -4.12 \mathrm{E}-04 & 6.48 \mathrm{E}-03 & -5.07 \mathrm{E}-05 & 9.07 \mathrm{E}-02 \\ 100 & 13.0 & 7.47 \mathrm{E}-05 & 2.81 \mathrm{E}-08 & 6.50 \mathrm{E}-03 & -4.12 \mathrm{E}-04 & 6.92 \mathrm{E}-03 & -5.49 \mathrm{E}-05 & 9.00 \mathrm{E}-02 \\ 100 & 12.0 & 8.06 \mathrm{E}-05 & 4.29 \mathrm{E}-08 & 7.02 \mathrm{E}-03 & -4.12 \mathrm{E}-04 & 7.43 \mathrm{E}-03 & -6.04 \mathrm{E}-05 & 8.92 \mathrm{E}-02 \\ 100 & 11.0 & 8.78 \mathrm{E}-05 & 5.41 \mathrm{E}-08 & 7.65 \mathrm{E}-03 & -4.12 \mathrm{E}-04 & 8.06 \mathrm{E}-03 & -6.64 \mathrm{E}-05 & 8.87 \mathrm{E}-02 \\ 100 & 10.0 & 9.62 \mathrm{E}-05 & 9.31 \mathrm{E}-08 & 8.38 \mathrm{E}-03 & -4.12 \mathrm{E}-04 & 8.79 \mathrm{E}-03 & -7.58 \mathrm{E}-05 & 8.80 \mathrm{E}-02 \\ 100 & 9.0 & 1.07 \mathrm{E}-04 & 1.05 \mathrm{E}-07 & 9.28 \mathrm{E}-03 & -4.12 \mathrm{E}-04 & 9.69 \mathrm{E}-03 & -8.41 \mathrm{E}-05 & 8.73 \mathrm{E}-02 \\ 100 & 8.0 & 1.19 \mathrm{E}-04 & 1.25 \mathrm{E}-07 & 1.04 \mathrm{E}-02 & -4.12 \mathrm{E}-04 & 1.08 \mathrm{E}-02 & -9.46 \mathrm{E}-05 & 8.63 \mathrm{E}-02 \\ 100 & 7.0 & 1.35 \mathrm{E}-04 & 1.15 \mathrm{E}-07 & 1.18 \mathrm{E}-02 & -4.12 \mathrm{E}-04 & 1.22 \mathrm{E}-02 & -1.05 \mathrm{E}-04 & 8.54 \mathrm{E}-02 \\ 100 & 6.0 & 1.56 \mathrm{E}-04 & 2.55 \mathrm{E}-07 & 1.36 \mathrm{E}-02 & -4.12 \mathrm{E}-04 & 1.40 \mathrm{E}-02 & -1.32 \mathrm{E}-04 & 8.42 \mathrm{E}-02 \\ 100 & 5.0 & 1.85 \mathrm{E}-04 & 2.46 \mathrm{E}-07 & 1.61 \mathrm{E}-02 & -4.12 \mathrm{E}-04 & 1.65 \mathrm{E}-02 & -1.52 \mathrm{E}-04 & 8.26 \mathrm{E}-02\end{array}$


Complex 7 ([ $\left.\left\{\mathrm{Mn}\left(\mathrm{CH}_{2} \mathrm{SiMe}_{3}\right)\left(\mu-\mathrm{CH}_{2} \mathrm{SiMe}_{3}\right)\right\}_{2}(\mu-\mathrm{dmpm})\right]$

Mass (mg): 49.5

Field

(Oe)

100

100

100

100

100

100

100

100

100

100

100

100

100

100

100

100

100

100

100

100

100

100

100

100

100

100

100

100

100

100

100

100

100

100

100

100

100

$100 \quad 115.0$

$100 \quad 110.0$

289.7

284.7

279.7

269.7

264.7

259.7

254.7

249.7

244.7

234.7

229.7

224.7

219.8

214.7

209.7

199.7

194.7
189.8

179.8

174.8

164.8

154.9

149.9

144.9

139.9

134.9

129.9

124.9
Mol. Wt. $\left(\mathrm{gmol}^{-1}\right): 594.86$

Temp (K) EMU $\quad$ STD $\quad \begin{gathered}\text { Chi } \\ (\mathrm{cm} 3 / \mathrm{mol})\end{gathered} \begin{gathered}\text { dia } \\ (\mathrm{cm} 3 / \mathrm{mol})\end{gathered}$ $300.0 \quad 5.04 \mathrm{E}-05 \quad 4.59 \mathrm{E}-09$

$294.7 \quad 5.04 \mathrm{E}-05 \quad 2.31 \mathrm{E}-08$

5.04E-05 2.14E-08

5.04E-05

$1.96 \mathrm{E}-08$

1.57E-08

5.03E-05

$1.92 \mathrm{E}-08$

5.03E-05

1.23E-08

5.02E-05

2.02E-08

5.02E-05

$1.82 \mathrm{E}-08$

5.01E-05

1.13E-08

$5.00 \mathrm{E}-05$

6.06E-09

8.01E-09

2.47E-08

4.97E-05

3.05E-08

$4.96 \mathrm{E}-05$

1.73E-08

4.95E-05

$1.51 \mathrm{E}-08$

5.31E-08

4.92E-05 3.83E-08

4.91E-05

$2.10 \mathrm{E}-08$

4.89E-05

1.33E-08

$4.88 \mathrm{E}-0$

1.12E-08

$1.21 \mathrm{E}-08$

4.86E-05

1.64E-08

4.85E-05

$1.40 \mathrm{E}-08$

4.83E-0

$1.48 \mathrm{E}-08$

4.81E-0

$1.88 \mathrm{E}-08$

4.79E-0

2.35E-08

4.77E-0

$1.18 \mathrm{E}-08$

4.75E-05

$1.08 \mathrm{E}-08$

4.71E-05 $1.41 \mathrm{E}-08$

4.68E-05 2.51E-08

4.66E-05 2.19E-08

4.63E-05

4.60E-0

2.44E-08

2.30E-08

4.57E-05 1.56E-08

4.53E-05 2.32E-08

4.49E-05 1.84E-08

4.45E-05 2.79E-08

4.41E-05 1.33E-08 (cm3/mol)

$6.06 \mathrm{E}-03$

$6.06 \mathrm{E}-03$

$6.06 \mathrm{E}-03$

$6.05 \mathrm{E}-03$

6.05E-03

6.04E-03

6.03E-03

6.02E-03

6.01E-03

$6.00 \mathrm{E}-03$

5.99E-03

5.98E-03

5.97E-03

5.95E-03

5.94E-03

5.94E-03

5.92E-03

5.90E-03

5.88E-03

$5.86 \mathrm{E}-03$

5.85E-03

5.83E-03

5.81E-03

$5.78 \mathrm{E}-03$

$5.76 \mathrm{E}-03$

5.74E-03

5.71E-03

$5.68 \mathrm{E}-03$

5.66E-03

5.63E-03

$5.59 \mathrm{E}-03$

$5.56 \mathrm{E}-03$

$5.53 \mathrm{E}-03$

5.49E-03

5.45E-03

5.40E-03

5.35E-03

$5.30 \mathrm{E}-03$
$-4.29 \mathrm{E}-04$

$-4.29 \mathrm{E}-04$

$-4.29 \mathrm{E}-04$

$-4.29 \mathrm{E}-04$

$-4.29 \mathrm{E}-04$

$-4.29 \mathrm{E}-04$

$-4.29 \mathrm{E}-04$

$-4.29 \mathrm{E}-04$

$-4.29 \mathrm{E}-04$

$-4.29 \mathrm{E}-04$

$-4.29 \mathrm{E}-04$

$-4.29 \mathrm{E}-04$

$-4.29 \mathrm{E}-04$

$-4.29 \mathrm{E}-04$

$-4.29 \mathrm{E}-04$

$-4.29 \mathrm{E}-04$

$-4.29 \mathrm{E}-04$

$-4.29 \mathrm{E}-04$

$-4.29 \mathrm{E}-04$

$-4.29 \mathrm{E}-04$

$-4.29 \mathrm{E}-04$

$-4.29 \mathrm{E}-04$

$-4.29 \mathrm{E}-04$

$-4.29 \mathrm{E}-04$

$-4.29 \mathrm{E}-04$

$-4.29 \mathrm{E}-04$

$-4.29 \mathrm{E}-04$

$-4.29 \mathrm{E}-04$

$-4.29 \mathrm{E}-04$

$-4.29 \mathrm{E}-04$

$-4.29 \mathrm{E}-04$

$-4.29 \mathrm{E}-04$

$-4.29 \mathrm{E}-04$

$-4.29 \mathrm{E}-04$

$-4.29 \mathrm{E}-04$

$-4.29 \mathrm{E}-04$

$-4.29 \mathrm{E}-04$

$-4.29 \mathrm{E}-04$

$-4.29 \mathrm{E}-04$
Cor Chi

(cm3/mol)

$6.48 \mathrm{E}-03$

$6.48 \mathrm{E}-03$

$6.49 \mathrm{E}-03$

$6.48 \mathrm{E}-03$

$6.48 \mathrm{E}-03$

$6.47 \mathrm{E}-03$

$6.47 \mathrm{E}-03$

$6.46 \mathrm{E}-03$

$6.45 \mathrm{E}-03$

6.44E-03

$6.43 \mathrm{E}-03$

6.42E-03

6.41E-03

$6.39 \mathrm{E}-03$

$6.38 \mathrm{E}-03$

6.37E-03

6.37E-03

6.35E-03

$6.33 \mathrm{E}-03$

$6.31 \mathrm{E}-03$

$6.29 \mathrm{E}-03$

$6.27 \mathrm{E}-03$

$6.26 \mathrm{E}-03$

$6.23 \mathrm{E}-03$

$6.21 \mathrm{E}-03$

6.19E-03

6.17E-03

6.14E-03

$6.11 \mathrm{E}-03$

$6.08 \mathrm{E}-03$

$6.05 \mathrm{E}-03-6.05 \mathrm{E}-05$

6.02E-03 -5.97E-05

5.99E-03 -5.97E-05

5.95E-03 -5.92E-05

5.92E-03 -5.79E-05

5.87E-03 -5.84E-05

5.83E-03 -5.73E-05

$5.78 \mathrm{E}-03 \quad-5.80 \mathrm{E}-05$

$5.72 \mathrm{E}-03 \quad-5.56 \mathrm{E}-05$

Chi*T

(cm3K/mol)

$1.95 \mathrm{E}+00$

$1.91 \mathrm{E}+00$

$1.88 \mathrm{E}+00$

$1.85 \mathrm{E}+00$

$1.81 \mathrm{E}+00$

$1.78 \mathrm{E}+00$

$1.74 \mathrm{E}+00$

$1.71 \mathrm{E}+00$

$1.67 \mathrm{E}+00$

$1.64 \mathrm{E}+00$

$1.60 \mathrm{E}+00$

$1.57 \mathrm{E}+00$

$1.54 \mathrm{E}+00$

$1.50 \mathrm{E}+00$

$1.47 \mathrm{E}+00$

$1.43 \mathrm{E}+00$

$1.40 \mathrm{E}+00$

$1.36 \mathrm{E}+00$

$1.33 \mathrm{E}+00$

$1.29 \mathrm{E}+00$

$1.26 \mathrm{E}+00$

$1.22 \mathrm{E}+00$

$1.19 \mathrm{E}+00$

$1.15 \mathrm{E}+00$

$1.12 \mathrm{E}+00$

$1.08 \mathrm{E}+00$

$1.05 \mathrm{E}+00$

$1.01 \mathrm{E}+00$

9.77E-01 


\begin{tabular}{|c|c|c|c|c|c|c|c|c|}
\hline 100 & 105.0 & $4.36 \mathrm{E}-05$ & 3.35E-08 & $5.24 \mathrm{E}-03$ & $-4.29 \mathrm{E}-04$ & $5.66 \mathrm{E}-03$ & $-5.75 \mathrm{E}-05$ & 5.95E-01 \\
\hline 100 & 100.0 & 4.31E-05 & $1.18 \mathrm{E}-07$ & $5.18 \mathrm{E}-03$ & $-4.29 \mathrm{E}-04$ & $5.61 \mathrm{E}-03$ & $-6.71 \mathrm{E}-05$ & $5.61 \mathrm{E}-01$ \\
\hline 100 & 99.0 & $4.31 \mathrm{E}-05$ & 2.27E-09 & $5.18 \mathrm{E}-03$ & $-4.29 \mathrm{E}-04$ & $5.61 \mathrm{E}-03$ & $-5.31 \mathrm{E}-05$ & $5.55 \mathrm{E}-01$ \\
\hline 100 & 97.9 & $4.30 \mathrm{E}-05$ & $8.23 \mathrm{E}-09$ & $5.17 \mathrm{E}-03$ & $-4.29 \mathrm{E}-04$ & $5.60 \mathrm{E}-03$ & $-5.37 \mathrm{E}-05$ & $5.48 \mathrm{E}-01$ \\
\hline 100 & 96.9 & $4.28 \mathrm{E}-05$ & $1.49 \mathrm{E}-08$ & $5.15 \mathrm{E}-03$ & $-4.29 \mathrm{E}-04$ & $5.57 \mathrm{E}-03$ & $-5.43 \mathrm{E}-05$ & $5.40 \mathrm{E}-01$ \\
\hline 100 & 95.9 & 4.27E-05 & 5.97E-09 & $5.13 \mathrm{E}-03$ & $-4.29 \mathrm{E}-04$ & $5.56 \mathrm{E}-03$ & $-5.31 \mathrm{E}-05$ & 5.33E-01 \\
\hline 100 & 94.9 & $4.26 \mathrm{E}-05$ & $2.21 \mathrm{E}-08$ & $5.12 \mathrm{E}-03$ & $-4.29 \mathrm{E}-04$ & $5.55 \mathrm{E}-03$ & $-5.49 \mathrm{E}-05$ & $5.27 \mathrm{E}-01$ \\
\hline 100 & 93.9 & $4.25 \mathrm{E}-05$ & 8.91E-09 & $5.10 \mathrm{E}-03$ & $-4.29 \mathrm{E}-04$ & $5.53 \mathrm{E}-03$ & $-5.31 \mathrm{E}-05$ & $5.19 \mathrm{E}-01$ \\
\hline 100 & 92.9 & 4.23E-05 & $3.20 \mathrm{E}-08$ & $5.09 \mathrm{E}-03$ & $-4.29 \mathrm{E}-04$ & $5.52 \mathrm{E}-03$ & $-5.58 \mathrm{E}-05$ & $5.12 \mathrm{E}-01$ \\
\hline 100 & 91.9 & $4.22 \mathrm{E}-05$ & $2.16 \mathrm{E}-08$ & $5.07 \mathrm{E}-03$ & $-4.29 \mathrm{E}-04$ & $5.50 \mathrm{E}-03$ & $-5.44 \mathrm{E}-05$ & $5.05 \mathrm{E}-01$ \\
\hline 100 & 90.9 & $4.21 \mathrm{E}-05$ & $1.37 \mathrm{E}-08$ & $5.05 \mathrm{E}-03$ & $-4.29 \mathrm{E}-04$ & $5.48 \mathrm{E}-03$ & $-5.32 \mathrm{E}-05$ & 4.98E-01 \\
\hline 100 & 89.9 & 4.19E-05 & 7.37E-09 & $5.04 \mathrm{E}-03$ & $-4.29 \mathrm{E}-04$ & $5.46 \mathrm{E}-03$ & $-5.23 \mathrm{E}-05$ & 4.91E-01 \\
\hline 100 & 88.9 & 4.17E-05 & $2.70 \mathrm{E}-08$ & $5.02 \mathrm{E}-03$ & $-4.29 \mathrm{E}-04$ & $5.45 \mathrm{E}-03$ & $-5.45 \mathrm{E}-05$ & 4.84E-01 \\
\hline 100 & 87.9 & 4.16E-05 & $3.14 \mathrm{E}-08$ & $5.00 \mathrm{E}-03$ & $-4.29 \mathrm{E}-04$ & $5.43 \mathrm{E}-03$ & $-5.48 \mathrm{E}-05$ & 4.77E-01 \\
\hline 100 & 86.9 & $4.14 \mathrm{E}-05$ & 2.03E-08 & $4.98 \mathrm{E}-03$ & $-4.29 \mathrm{E}-04$ & $5.41 \mathrm{E}-03$ & $-5.33 \mathrm{E}-05$ & 4.70E-01 \\
\hline 100 & 85.9 & 4.13E-05 & 2.34E-08 & $4.96 \mathrm{E}-03$ & $-4.29 \mathrm{E}-04$ & 5.39E-03 & $-5.34 \mathrm{E}-05$ & 4.63E-01 \\
\hline 100 & 84.9 & $4.11 \mathrm{E}-05$ & $2.40 \mathrm{E}-08$ & $4.94 \mathrm{E}-03$ & $-4.29 \mathrm{E}-04$ & $5.37 \mathrm{E}-03$ & $-5.33 \mathrm{E}-05$ & 4.56E-01 \\
\hline 100 & 83.9 & 4.09E-05 & $2.68 \mathrm{E}-08$ & $4.92 \mathrm{E}-03$ & $-4.29 \mathrm{E}-04$ & $5.35 \mathrm{E}-03$ & $-5.34 \mathrm{E}-05$ & 4.49E-01 \\
\hline 100 & 82.9 & 4.07E-05 & $3.23 \mathrm{E}-08$ & $4.90 \mathrm{E}-03$ & $-4.29 \mathrm{E}-04$ & $5.32 \mathrm{E}-03$ & $-5.39 \mathrm{E}-05$ & 4.41E-01 \\
\hline 100 & 81.9 & $4.06 \mathrm{E}-05$ & $3.47 \mathrm{E}-08$ & 4.87E-03 & $-4.29 \mathrm{E}-04$ & $5.30 \mathrm{E}-03$ & $-5.39 \mathrm{E}-05$ & 4.34E-01 \\
\hline 100 & 80.9 & $4.04 \mathrm{E}-05$ & $2.00 \mathrm{E}-08$ & $4.85 \mathrm{E}-03$ & $-4.29 \mathrm{E}-04$ & $5.28 \mathrm{E}-03$ & $-5.19 \mathrm{E}-05$ & 4.27E-01 \\
\hline 100 & 79.9 & 4.02E-05 & 4.77E-08 & $4.83 \mathrm{E}-03$ & $-4.29 \mathrm{E}-04$ & $5.25 \mathrm{E}-03$ & $-5.50 \mathrm{E}-05$ & 4.20E-01 \\
\hline 100 & 78.9 & 3.99E-05 & 2.79E-08 & $4.80 \mathrm{E}-03$ & $-4.29 \mathrm{E}-04$ & $5.23 \mathrm{E}-03$ & $-5.24 \mathrm{E}-05$ & 4.13E-01 \\
\hline 100 & 78.0 & $3.97 \mathrm{E}-05$ & 2.79E-08 & $4.78 \mathrm{E}-03$ & $-4.29 \mathrm{E}-04$ & $5.20 \mathrm{E}-03$ & $-5.21 \mathrm{E}-05$ & 4.06E-01 \\
\hline 100 & 77.0 & $3.95 \mathrm{E}-05$ & 3.13E-08 & $4.75 \mathrm{E}-03$ & $-4.29 \mathrm{E}-04$ & $5.18 \mathrm{E}-03$ & $-5.23 \mathrm{E}-05$ & 3.98E-01 \\
\hline 100 & 76.0 & 3.93E-05 & $2.61 \mathrm{E}-08$ & $4.72 \mathrm{E}-03$ & $-4.29 \mathrm{E}-04$ & $5.15 \mathrm{E}-03$ & $-5.13 \mathrm{E}-05$ & $3.91 \mathrm{E}-01$ \\
\hline 100 & 75.0 & $3.91 \mathrm{E}-05$ & $3.29 \mathrm{E}-08$ & $4.70 \mathrm{E}-03$ & $-4.29 \mathrm{E}-04$ & $5.12 \mathrm{E}-03$ & $-5.19 \mathrm{E}-05$ & $3.84 \mathrm{E}-01$ \\
\hline 100 & 74.0 & $3.88 \mathrm{E}-05$ & $2.75 \mathrm{E}-08$ & 4.67E-03 & $-4.29 \mathrm{E}-04$ & $5.09 \mathrm{E}-03$ & $-5.09 \mathrm{E}-05$ & 3.77E-01 \\
\hline 100 & 73.0 & $3.86 \mathrm{E}-05$ & $3.34 \mathrm{E}-08$ & $4.63 \mathrm{E}-03$ & $-4.29 \mathrm{E}-04$ & $5.06 \mathrm{E}-03$ & $-5.13 \mathrm{E}-05$ & $3.70 \mathrm{E}-01$ \\
\hline 100 & 72.0 & $3.83 \mathrm{E}-05$ & $2.66 \mathrm{E}-08$ & $4.60 \mathrm{E}-03$ & $-4.29 \mathrm{E}-04$ & $5.03 \mathrm{E}-03$ & $-5.02 \mathrm{E}-05$ & 3.62E-01 \\
\hline 100 & 71.0 & $3.80 \mathrm{E}-05$ & 4.62E-08 & 4.57E-03 & $-4.29 \mathrm{E}-04$ & $5.00 \mathrm{E}-03$ & $-5.22 \mathrm{E}-05$ & 3.55E-01 \\
\hline 100 & 70.0 & $3.78 \mathrm{E}-05$ & $4.98 \mathrm{E}-08$ & $4.54 \mathrm{E}-03$ & $-4.29 \mathrm{E}-04$ & 4.97E-03 & $-5.24 \mathrm{E}-05$ & $3.48 \mathrm{E}-01$ \\
\hline 100 & 69.0 & $3.75 \mathrm{E}-05$ & $3.79 \mathrm{E}-08$ & $4.51 \mathrm{E}-03$ & $-4.29 \mathrm{E}-04$ & 4.94E-03 & $-5.06 \mathrm{E}-05$ & $3.41 \mathrm{E}-01$ \\
\hline 100 & 68.0 & 3.72E-05 & $2.74 \mathrm{E}-08$ & 4.47E-03 & $-4.29 \mathrm{E}-04$ & $4.90 \mathrm{E}-03$ & $-4.89 \mathrm{E}-05$ & 3.33E-01 \\
\hline 100 & 67.0 & $3.69 \mathrm{E}-05$ & $3.94 \mathrm{E}-08$ & 4.43E-03 & $-4.29 \mathrm{E}-04$ & $4.86 \mathrm{E}-03$ & $-5.00 \mathrm{E}-05$ & $3.26 \mathrm{E}-01$ \\
\hline 100 & 66.0 & $3.66 \mathrm{E}-05$ & 7.97E-09 & $4.40 \mathrm{E}-03$ & $-4.29 \mathrm{E}-04$ & $4.83 \mathrm{E}-03$ & $-4.59 \mathrm{E}-05$ & $3.19 \mathrm{E}-01$ \\
\hline 100 & 65.0 & $3.63 \mathrm{E}-05$ & $6.41 \mathrm{E}-09$ & $4.36 \mathrm{E}-03$ & $-4.29 \mathrm{E}-04$ & 4.79E-03 & $-4.53 \mathrm{E}-05$ & $3.12 \mathrm{E}-01$ \\
\hline 100 & 64.0 & $3.60 \mathrm{E}-05$ & $1.18 \mathrm{E}-09$ & 4.32E-03 & $-4.29 \mathrm{E}-04$ & $4.75 \mathrm{E}-03$ & $-4.42 \mathrm{E}-05$ & $3.04 \mathrm{E}-01$ \\
\hline 100 & 63.0 & $3.56 \mathrm{E}-05$ & 2.15E-09 & $4.28 \mathrm{E}-03$ & $-4.29 \mathrm{E}-04$ & $4.71 \mathrm{E}-03$ & $-4.39 \mathrm{E}-05$ & 2.97E-01 \\
\hline 100 & 62.0 & $3.53 \mathrm{E}-05$ & 8.03E-09 & $4.24 \mathrm{E}-03$ & $-4.29 \mathrm{E}-04$ & $4.66 \mathrm{E}-03$ & $-4.42 \mathrm{E}-05$ & $2.89 \mathrm{E}-01$ \\
\hline 100 & 61.0 & $3.49 \mathrm{E}-05$ & $8.45 \mathrm{E}-09$ & 4.19E-03 & $-4.29 \mathrm{E}-04$ & $4.62 \mathrm{E}-03$ & $-4.38 \mathrm{E}-05$ & $2.82 \mathrm{E}-01$ \\
\hline 100 & 60.0 & $3.45 \mathrm{E}-05$ & $6.20 \mathrm{E}-09$ & 4.14E-03 & $-4.29 \mathrm{E}-04$ & $4.57 \mathrm{E}-03$ & $-4.30 \mathrm{E}-05$ & 2.75E-01 \\
\hline 100 & 59.0 & $3.41 \mathrm{E}-05$ & $4.80 \mathrm{E}-09$ & $4.10 \mathrm{E}-03$ & $-4.29 \mathrm{E}-04$ & $4.52 \mathrm{E}-03$ & $-4.24 \mathrm{E}-05$ & $2.67 \mathrm{E}-01$ \\
\hline
\end{tabular}




\begin{tabular}{|c|c|c|c|c|c|c|c|c|}
\hline 100 & 58.0 & 3.37E-05 & $3.58 \mathrm{E}-09$ & $4.05 \mathrm{E}-03$ & $-4.29 \mathrm{E}-04$ & $4.47 \mathrm{E}-03$ & $-4.17 \mathrm{E}-05$ & $2.60 \mathrm{E}-01$ \\
\hline 100 & 57.0 & $3.32 \mathrm{E}-05$ & $8.45 \mathrm{E}-09$ & $4.00 \mathrm{E}-03$ & $-4.29 \mathrm{E}-04$ & $4.42 \mathrm{E}-03$ & $-4.18 \mathrm{E}-05$ & $2.52 \mathrm{E}-01$ \\
\hline 100 & 56.0 & $3.28 \mathrm{E}-05$ & 2.71E-09 & $3.94 \mathrm{E}-03$ & $-4.29 \mathrm{E}-04$ & 4.37E-03 & $-4.06 \mathrm{E}-05$ & $2.45 \mathrm{E}-01$ \\
\hline 100 & 55.0 & $3.24 \mathrm{E}-05$ & $5.47 \mathrm{E}-09$ & $3.89 \mathrm{E}-03$ & $-4.29 \mathrm{E}-04$ & 4.32E-03 & $-4.03 \mathrm{E}-05$ & $2.38 \mathrm{E}-01$ \\
\hline 100 & 54.0 & 3.19E-05 & $1.49 \mathrm{E}-08$ & $3.83 \mathrm{E}-03$ & $-4.29 \mathrm{E}-04$ & $4.26 \mathrm{E}-03$ & $-4.09 \mathrm{E}-05$ & $2.30 \mathrm{E}-01$ \\
\hline 100 & 53.0 & $3.14 \mathrm{E}-05$ & 4.53E-09 & $3.78 \mathrm{E}-03$ & $-4.29 \mathrm{E}-04$ & 4.20E-03 & $-3.91 \mathrm{E}-05$ & $2.23 \mathrm{E}-01$ \\
\hline 100 & 52.0 & $3.09 \mathrm{E}-05$ & 3.81E-09 & $3.72 \mathrm{E}-03$ & $-4.29 \mathrm{E}-04$ & 4.15E-03 & $-3.84 \mathrm{E}-05$ & $2.16 \mathrm{E}-01$ \\
\hline 100 & 51.0 & 3.04E-05 & $5.96 \mathrm{E}-10$ & $3.66 \mathrm{E}-03$ & $-4.29 \mathrm{E}-04$ & $4.08 \mathrm{E}-03$ & $-3.74 \mathrm{E}-05$ & $2.08 \mathrm{E}-01$ \\
\hline 100 & 50.0 & 2.99E-05 & $5.66 \mathrm{E}-09$ & $3.59 \mathrm{E}-03$ & $-4.29 \mathrm{E}-04$ & 4.02E-03 & $-3.74 \mathrm{E}-05$ & $2.01 \mathrm{E}-01$ \\
\hline 100 & 49.0 & 2.94E-05 & 8.99E-09 & $3.53 \mathrm{E}-03$ & $-4.29 \mathrm{E}-04$ & $3.96 \mathrm{E}-03$ & $-3.71 \mathrm{E}-05$ & $1.94 \mathrm{E}-01$ \\
\hline 100 & 48.0 & $2.88 \mathrm{E}-05$ & 8.10E-09 & $3.46 \mathrm{E}-03$ & $-4.29 \mathrm{E}-04$ & $3.89 \mathrm{E}-03$ & $-3.63 \mathrm{E}-05$ & $1.87 \mathrm{E}-01$ \\
\hline 100 & 47.0 & $2.86 \mathrm{E}-05$ & $8.71 \mathrm{E}-09$ & $3.43 \mathrm{E}-03$ & $-4.29 \mathrm{E}-04$ & $3.86 \mathrm{E}-03$ & $-3.61 \mathrm{E}-05$ & $1.82 \mathrm{E}-01$ \\
\hline 100 & 46.0 & $2.80 \mathrm{E}-05$ & $1.14 \mathrm{E}-08$ & $3.37 \mathrm{E}-03$ & $-4.29 \mathrm{E}-04$ & $3.80 \mathrm{E}-03$ & $-3.58 \mathrm{E}-05$ & $1.75 \mathrm{E}-01$ \\
\hline 100 & 45.0 & $2.75 \mathrm{E}-05$ & 6.02E-09 & $3.30 \mathrm{E}-03$ & $-4.29 \mathrm{E}-04$ & $3.73 \mathrm{E}-03$ & $-3.44 \mathrm{E}-05$ & $1.68 \mathrm{E}-01$ \\
\hline 100 & 44.0 & 2.69E-05 & $1.01 \mathrm{E}-08$ & $3.23 \mathrm{E}-03$ & $-4.29 \mathrm{E}-04$ & $3.66 \mathrm{E}-03$ & $-3.42 \mathrm{E}-05$ & $1.61 \mathrm{E}-01$ \\
\hline 100 & 43.0 & 2.63E-05 & $3.80 \mathrm{E}-09$ & $3.16 \mathrm{E}-03$ & $-4.29 \mathrm{E}-04$ & $3.59 \mathrm{E}-03$ & $-3.27 \mathrm{E}-05$ & $1.54 \mathrm{E}-01$ \\
\hline 100 & 42.0 & $2.57 \mathrm{E}-05$ & 2.65E-09 & $3.09 \mathrm{E}-03$ & $-4.29 \mathrm{E}-04$ & $3.52 \mathrm{E}-03$ & $-3.19 \mathrm{E}-05$ & $1.48 \mathrm{E}-01$ \\
\hline 100 & 41.0 & $2.52 \mathrm{E}-05$ & 7.52E-09 & $3.02 \mathrm{E}-03$ & $-4.29 \mathrm{E}-04$ & $3.45 \mathrm{E}-03$ & $-3.18 \mathrm{E}-05$ & $1.42 \mathrm{E}-01$ \\
\hline 100 & 40.0 & $2.46 \mathrm{E}-05$ & 7.09E-09 & $2.96 \mathrm{E}-03$ & $-4.29 \mathrm{E}-04$ & 3.39E-03 & $-3.10 \mathrm{E}-05$ & $1.35 \mathrm{E}-01$ \\
\hline 100 & 39.0 & $2.40 \mathrm{E}-05$ & 2.72E-09 & 2.89E-03 & $-4.29 \mathrm{E}-04$ & $3.32 \mathrm{E}-03$ & $-2.98 \mathrm{E}-05$ & $1.29 \mathrm{E}-01$ \\
\hline 100 & 38.0 & 2.35E-05 & 6.87E-09 & $2.82 \mathrm{E}-03$ & $-4.29 \mathrm{E}-04$ & $3.25 \mathrm{E}-03$ & $-2.97 \mathrm{E}-05$ & $1.24 \mathrm{E}-01$ \\
\hline 100 & 37.0 & $2.30 \mathrm{E}-05$ & $1.92 \mathrm{E}-09$ & $2.76 \mathrm{E}-03$ & $-4.29 \mathrm{E}-04$ & $3.19 \mathrm{E}-03$ & $-2.84 \mathrm{E}-05$ & $1.18 \mathrm{E}-01$ \\
\hline 100 & 36.0 & $2.25 \mathrm{E}-05$ & 5.44E-09 & $2.70 \mathrm{E}-03$ & $-4.29 \mathrm{E}-04$ & $3.13 \mathrm{E}-03$ & $-2.82 \mathrm{E}-05$ & $1.13 \mathrm{E}-01$ \\
\hline 100 & 35.0 & $2.20 \mathrm{E}-05$ & $3.96 \mathrm{E}-09$ & $2.64 \mathrm{E}-03$ & $-4.29 \mathrm{E}-04$ & $3.07 \mathrm{E}-03$ & $-2.74 \mathrm{E}-05$ & $1.07 \mathrm{E}-01$ \\
\hline 100 & 34.0 & $2.15 \mathrm{E}-05$ & $6.45 \mathrm{E}-09$ & $2.59 \mathrm{E}-03$ & $-4.29 \mathrm{E}-04$ & $3.02 \mathrm{E}-03$ & $-2.72 \mathrm{E}-05$ & $1.03 \mathrm{E}-01$ \\
\hline 100 & 33.0 & $2.11 \mathrm{E}-05$ & $3.02 \mathrm{E}-09$ & $2.54 \mathrm{E}-03$ & $-4.29 \mathrm{E}-04$ & $2.96 \mathrm{E}-03$ & $-2.62 \mathrm{E}-05$ & $9.78 \mathrm{E}-02$ \\
\hline 100 & 32.0 & 2.07E-05 & $8.80 \mathrm{E}-10$ & $2.49 \mathrm{E}-03$ & $-4.29 \mathrm{E}-04$ & $2.92 \mathrm{E}-03$ & $-2.55 \mathrm{E}-05$ & $9.34 \mathrm{E}-02$ \\
\hline 100 & 31.0 & 2.04E-05 & $5.24 \mathrm{E}-09$ & $2.45 \mathrm{E}-03$ & $-4.29 \mathrm{E}-04$ & $2.88 \mathrm{E}-03$ & $-2.57 \mathrm{E}-05$ & $8.93 \mathrm{E}-02$ \\
\hline 100 & 30.0 & $2.02 \mathrm{E}-05$ & 4.97E-09 & $2.42 \mathrm{E}-03$ & $-4.29 \mathrm{E}-04$ & $2.85 \mathrm{E}-03$ & $-2.53 \mathrm{E}-05$ & $8.55 \mathrm{E}-02$ \\
\hline 100 & 29.0 & $2.00 \mathrm{E}-05$ & 4.20E-09 & $2.40 \mathrm{E}-03$ & $-4.29 \mathrm{E}-04$ & $2.83 \mathrm{E}-03$ & $-2.50 \mathrm{E}-05$ & $8.19 \mathrm{E}-02$ \\
\hline 100 & 28.0 & $1.98 \mathrm{E}-05$ & $1.28 \mathrm{E}-08$ & $2.38 \mathrm{E}-03$ & $-4.29 \mathrm{E}-04$ & $2.81 \mathrm{E}-03$ & $-2.59 \mathrm{E}-05$ & $7.87 \mathrm{E}-02$ \\
\hline 100 & 27.0 & $1.98 \mathrm{E}-05$ & 5.13E-09 & $2.38 \mathrm{E}-03$ & $-4.29 \mathrm{E}-04$ & $2.81 \mathrm{E}-03$ & $-2.49 \mathrm{E}-05$ & 7.57E-02 \\
\hline 100 & 26.0 & $1.98 \mathrm{E}-05$ & 3.72E-09 & $2.38 \mathrm{E}-03$ & $-4.29 \mathrm{E}-04$ & $2.81 \mathrm{E}-03$ & $-2.47 \mathrm{E}-05$ & $7.30 \mathrm{E}-02$ \\
\hline 100 & 25.0 & $2.00 \mathrm{E}-05$ & $5.67 \mathrm{E}-09$ & $2.40 \mathrm{E}-03$ & $-4.29 \mathrm{E}-04$ & $2.83 \mathrm{E}-03$ & $-2.52 \mathrm{E}-05$ & $7.06 \mathrm{E}-02$ \\
\hline 100 & 24.0 & $2.02 \mathrm{E}-05$ & $6.62 \mathrm{E}-09$ & $2.42 \mathrm{E}-03$ & $-4.29 \mathrm{E}-04$ & $2.85 \mathrm{E}-03$ & $-2.55 \mathrm{E}-05$ & $6.85 \mathrm{E}-02$ \\
\hline 100 & 23.0 & $2.05 \mathrm{E}-05$ & $3.12 \mathrm{E}-09$ & $2.47 \mathrm{E}-03$ & $-4.29 \mathrm{E}-04$ & $2.89 \mathrm{E}-03$ & $-2.55 \mathrm{E}-05$ & $6.66 \mathrm{E}-02$ \\
\hline 100 & 22.0 & $2.10 \mathrm{E}-05$ & $5.23 \mathrm{E}-09$ & $2.52 \mathrm{E}-03$ & $-4.29 \mathrm{E}-04$ & $2.95 \mathrm{E}-03$ & $-2.63 \mathrm{E}-05$ & $6.49 \mathrm{E}-02$ \\
\hline 100 & 21.0 & $2.15 \mathrm{E}-05$ & 3.72E-09 & $2.59 \mathrm{E}-03$ & $-4.29 \mathrm{E}-04$ & $3.02 \mathrm{E}-03$ & $-2.69 \mathrm{E}-05$ & $6.34 \mathrm{E}-02$ \\
\hline 100 & 20.0 & 2.23E-05 & $3.28 \mathrm{E}-09$ & $2.68 \mathrm{E}-03$ & $-4.29 \mathrm{E}-04$ & $3.11 \mathrm{E}-03$ & $-2.77 \mathrm{E}-05$ & $6.21 \mathrm{E}-02$ \\
\hline 100 & 19.0 & $2.31 \mathrm{E}-05$ & 3.30E-09 & $2.78 \mathrm{E}-03$ & $-4.29 \mathrm{E}-04$ & $3.21 \mathrm{E}-03$ & $-2.88 \mathrm{E}-05$ & $6.10 \mathrm{E}-02$ \\
\hline 100 & 18.0 & $2.42 \mathrm{E}-05$ & $1.23 \mathrm{E}-08$ & $2.90 \mathrm{E}-03$ & $-4.29 \mathrm{E}-04$ & $3.33 \mathrm{E}-03$ & $-3.11 \mathrm{E}-05$ & $6.00 \mathrm{E}-02$ \\
\hline 100 & 17.0 & $2.54 \mathrm{E}-05$ & 3.02E-09 & $3.05 \mathrm{E}-03$ & $-4.29 \mathrm{E}-04$ & $3.48 \mathrm{E}-03$ & $-3.15 \mathrm{E}-05$ & $5.91 \mathrm{E}-02$ \\
\hline 100 & 16.0 & 2.67E-05 & $6.82 \mathrm{E}-09$ & $3.21 \mathrm{E}-03$ & $-4.29 \mathrm{E}-04$ & $3.64 \mathrm{E}-03$ & $-3.36 \mathrm{E}-05$ & $5.83 \mathrm{E}-02$ \\
\hline
\end{tabular}




$\begin{array}{lllllllll}100 & 15.0 & 2.84 \mathrm{E}-05 & 7.22 \mathrm{E}-09 & 3.41 \mathrm{E}-03 & -4.29 \mathrm{E}-04 & 3.84 \mathrm{E}-03 & -3.57 \mathrm{E}-05 & 5.76 \mathrm{E}-02 \\ 100 & 14.0 & 3.02 \mathrm{E}-05 & 6.04 \mathrm{E}-09 & 3.63 \mathrm{E}-03 & -4.29 \mathrm{E}-04 & 4.06 \mathrm{E}-03 & -3.78 \mathrm{E}-05 & 5.68 \mathrm{E}-02 \\ 100 & 13.0 & 3.23 \mathrm{E}-05 & 8.44 \mathrm{E}-09 & 3.88 \mathrm{E}-03 & -4.29 \mathrm{E}-04 & 4.31 \mathrm{E}-03 & -4.07 \mathrm{E}-05 & 5.61 \mathrm{E}-02 \\ 100 & 12.0 & 3.48 \mathrm{E}-05 & 3.15 \mathrm{E}-09 & 4.18 \mathrm{E}-03 & -4.29 \mathrm{E}-04 & 4.61 \mathrm{E}-03 & -4.30 \mathrm{E}-05 & 5.53 \mathrm{E}-02 \\ 100 & 11.0 & 3.78 \mathrm{E}-05 & 1.39 \mathrm{E}-08 & 4.54 \mathrm{E}-03 & -4.29 \mathrm{E}-04 & 4.97 \mathrm{E}-03 & -4.80 \mathrm{E}-05 & 5.47 \mathrm{E}-02 \\ 100 & 10.0 & 4.13 \mathrm{E}-05 & 2.51 \mathrm{E}-08 & 4.96 \mathrm{E}-03 & -4.29 \mathrm{E}-04 & 5.39 \mathrm{E}-03 & -5.37 \mathrm{E}-05 & 5.39 \mathrm{E}-02 \\ 100 & 9.0 & 4.56 \mathrm{E}-05 & 3.05 \mathrm{E}-08 & 5.48 \mathrm{E}-03 & -4.29 \mathrm{E}-04 & 5.91 \mathrm{E}-03 & -5.96 \mathrm{E}-05 & 5.32 \mathrm{E}-02 \\ 100 & 8.0 & 5.08 \mathrm{E}-05 & 4.30 \mathrm{E}-08 & 6.11 \mathrm{E}-03 & -4.29 \mathrm{E}-04 & 6.54 \mathrm{E}-03 & -6.75 \mathrm{E}-05 & 5.23 \mathrm{E}-02 \\ 100 & 7.0 & 5.75 \mathrm{E}-05 & 6.28 \mathrm{E}-08 & 6.91 \mathrm{E}-03 & -4.29 \mathrm{E}-04 & 7.34 \mathrm{E}-03 & -7.81 \mathrm{E}-05 & 5.14 \mathrm{E}-02 \\ 100 & 6.0 & 6.63 \mathrm{E}-05 & 7.37 \mathrm{E}-08 & 7.96 \mathrm{E}-03 & -4.29 \mathrm{E}-04 & 8.39 \mathrm{E}-03 & -9.02 \mathrm{E}-05 & 5.04 \mathrm{E}-02 \\ 100 & 5.0 & 7.83 \mathrm{E}-05 & 7.25 \mathrm{E}-08 & 9.41 \mathrm{E}-03 & -4.29 \mathrm{E}-04 & 9.84 \mathrm{E}-03 & -1.05 \mathrm{E}-04 & 4.92 \mathrm{E}-02\end{array}$


Complex $8\left(\left[\left\{\mathrm{Mn}\left(\mathrm{CH}_{2} \mathrm{CMe}_{3}\right)\left(\mu-\mathrm{CH}_{2} \mathrm{CMe}_{3}\right)\right\}_{2}(\mu-\mathrm{dmpm})\right]\right.$

Mass (mg): 67.8

Field

\begin{tabular}{|c|c|c|c|c|c|c|c|c|}
\hline $\begin{array}{l}\text { Field } \\
(\mathrm{Oe})\end{array}$ & Гemp (K) & EMU & STD & $\begin{array}{c}\text { Chi } \\
\text { (cm3/mol) }\end{array}$ & $\begin{array}{c}\mathrm{dia} \\
(\mathrm{cm} 3 / \mathrm{mol})\end{array}$ & $\begin{array}{l}\text { Cor Chi } \\
(\mathrm{cm} 3 / \mathrm{mol})\end{array}$ & esd & $\begin{array}{c}\text { Chi*T } \\
(\mathrm{cm} 3 \mathrm{~K} / \mathrm{mol})\end{array}$ \\
\hline 0000 & 300.0 & 8.04E-03 & $6.21 \mathrm{E}-06$ & $6.29 \mathrm{E}-03$ & $-4.01 \mathrm{E}-04$ & $6.69 \mathrm{E}-03$ & $-5.16 \mathrm{E}-05$ & $2.01 \mathrm{E}+00$ \\
\hline 10000 & 294.7 & 8.03E-03 & $1.96 \mathrm{E}-06$ & $6.28 \mathrm{E}-03$ & $-4.01 \mathrm{E}-04$ & $6.68 \mathrm{E}-03$ & $-4.82 \mathrm{E}-05$ & $1.97 \mathrm{E}+00$ \\
\hline 10000 & 289.7 & 8.01E-03 & $9.98 \mathrm{E}-07$ & $6.27 \mathrm{E}-03$ & $-4.01 \mathrm{E}-04$ & $6.67 \mathrm{E}-03$ & $-4.74 \mathrm{E}-05$ & $1.93 \mathrm{E}+00$ \\
\hline 10000 & 284.6 & 8.00E-03 & $1.27 \mathrm{E}-06$ & $6.26 \mathrm{E}-03$ & $-4.01 \mathrm{E}-04$ & $6.66 \mathrm{E}-03$ & $-4.75 \mathrm{E}-05$ & $1.90 \mathrm{E}+00$ \\
\hline 10000 & 279.6 & 7.99E-03 & 8.67E-07 & $6.25 \mathrm{E}-03$ & $-4.01 \mathrm{E}-04$ & $6.65 \mathrm{E}-03$ & $-4.71 \mathrm{E}-05$ & $1.86 \mathrm{E}+00$ \\
\hline 10000 & 274.6 & $7.98 \mathrm{E}-03$ & $1.16 \mathrm{E}-06$ & $6.24 \mathrm{E}-03$ & $-4.01 \mathrm{E}-04$ & $6.64 \mathrm{E}-03$ & $-4.73 \mathrm{E}-05$ & $1.82 \mathrm{E}+00$ \\
\hline 10000 & 269.6 & 7.97E-03 & $1.23 \mathrm{E}-06$ & $6.23 \mathrm{E}-03$ & $-4.01 \mathrm{E}-04$ & $6.63 \mathrm{E}-03$ & $-4.73 \mathrm{E}-05$ & $1.79 \mathrm{E}+00$ \\
\hline 10000 & 264.6 & 7.95E-03 & $9.10 \mathrm{E}-07$ & $6.22 \mathrm{E}-03$ & $-4.01 \mathrm{E}-04$ & $6.62 \mathrm{E}-03$ & $-4.70 \mathrm{E}-05$ & $1.75 \mathrm{E}+00$ \\
\hline 10000 & 259.7 & 7.94E-03 & $1.02 \mathrm{E}-06$ & $6.21 \mathrm{E}-03$ & $-4.01 \mathrm{E}-04$ & $6.61 \mathrm{E}-03$ & $-4.70 \mathrm{E}-05$ & $1.72 \mathrm{E}+00$ \\
\hline 10000 & 254.7 & 7.92E-03 & $5.18 \mathrm{E}-07$ & $6.20 \mathrm{E}-03$ & $-4.01 \mathrm{E}-04$ & $6.60 \mathrm{E}-03$ & $-4.65 \mathrm{E}-05$ & $1.68 \mathrm{E}+00$ \\
\hline 10000 & 249.7 & 7.91E-03 & $6.37 \mathrm{E}-07$ & $6.19 \mathrm{E}-03$ & $-4.01 \mathrm{E}-04$ & $6.59 \mathrm{E}-03$ & $-4.65 \mathrm{E}-05$ & $1.65 \mathrm{E}+00$ \\
\hline 10000 & 244.7 & $7.90 \mathrm{E}-03$ & 8.63E-07 & $6.18 \mathrm{E}-03$ & $-4.01 \mathrm{E}-04$ & $6.58 \mathrm{E}-03$ & $-4.66 \mathrm{E}-05$ & $1.61 \mathrm{E}+00$ \\
\hline 10000 & 239.7 & $7.88 \mathrm{E}-03$ & $5.35 \mathrm{E}-07$ & $6.17 \mathrm{E}-03$ & $-4.01 \mathrm{E}-04$ & $6.57 \mathrm{E}-03$ & $-4.62 \mathrm{E}-05$ & $1.57 \mathrm{E}+00$ \\
\hline 10000 & 234.7 & 7.87E-03 & $8.58 \mathrm{E}-07$ & $6.16 \mathrm{E}-03$ & $-4.01 \mathrm{E}-04$ & $6.56 \mathrm{E}-03$ & $-4.64 \mathrm{E}-05$ & $1.54 \mathrm{E}+00$ \\
\hline 10000 & 229.7 & $7.85 \mathrm{E}-03$ & $7.44 \mathrm{E}-07$ & $6.15 \mathrm{E}-03$ & $-4.01 \mathrm{E}-04$ & $6.55 \mathrm{E}-03$ & $-4.62 \mathrm{E}-05$ & $1.50 \mathrm{E}+00$ \\
\hline 10000 & 224.7 & 7.84E-03 & $9.83 \mathrm{E}-07$ & $6.13 \mathrm{E}-03$ & $-4.01 \mathrm{E}-04$ & $6.53 \mathrm{E}-03$ & $-4.63 \mathrm{E}-05$ & $1.47 \mathrm{E}+00$ \\
\hline 10000 & 219.7 & $7.82 \mathrm{E}-03$ & $4.48 \mathrm{E}-07$ & $6.12 \mathrm{E}-03$ & $-4.01 \mathrm{E}-04$ & $6.52 \mathrm{E}-03$ & E-05 & $3 \mathrm{E}+00$ \\
\hline 10000 & 214.7 & $7.81 \mathrm{E}-03$ & $3.40 \mathrm{E}-07$ & $6.11 \mathrm{E}-03$ & $-4.01 \mathrm{E}-04$ & $6.51 \mathrm{E}-03$ & $-4.57 \mathrm{E}-05$ & $1.40 \mathrm{E}+00$ \\
\hline 10000 & 209.7 & $7.80 \mathrm{E}-03$ & 7.59E-07 & $6.10 \mathrm{E}-03$ & $-4.01 \mathrm{E}-04$ & $6.50 \mathrm{E}-03$ & $-4.59 \mathrm{E}-05$ & $1.36 \mathrm{E}+00$ \\
\hline 10000 & 204.7 & $7.78 \mathrm{E}-03$ & $7.51 \mathrm{E}-07$ & $6.09 \mathrm{E}-03$ & $-4.01 \mathrm{E}-04$ & $6.49 \mathrm{E}-03$ & $-4.58 \mathrm{E}-05$ & $1.33 \mathrm{E}+00$ \\
\hline 10000 & 199.7 & 7.77E-03 & $5.49 \mathrm{E}-07$ & $6.08 \mathrm{E}-03$ & $-4.01 \mathrm{E}-04$ & $6.48 \mathrm{E}-03$ & $-4.56 \mathrm{E}-05$ & $1.29 \mathrm{E}+00$ \\
\hline 10000 & 194.7 & $7.83 \mathrm{E}-03$ & $2.72 \mathrm{E}-06$ & $6.13 \mathrm{E}-03$ & $-4.01 \mathrm{E}-04$ & $6.53 \mathrm{E}-03$ & $-4.77 \mathrm{E}-05$ & $1.27 \mathrm{E}+00$ \\
\hline 10000 & 189.8 & 7.82E-03 & $1.23 \mathrm{E}-06$ & $6.12 \mathrm{E}-03$ & $-4.01 \mathrm{E}-04$ & $6.52 \mathrm{E}-03$ & $-4.65 \mathrm{E}-05$ & $1.24 \mathrm{E}+00$ \\
\hline 10000 & 184.8 & $7.81 \mathrm{E}-03$ & $1.50 \mathrm{E}-06$ & $6.11 \mathrm{E}-03$ & $-4.01 \mathrm{E}-04$ & $6.51 \mathrm{E}-03$ & $-4.66 \mathrm{E}-05$ & $1.20 \mathrm{E}+00$ \\
\hline 10000 & 179.8 & $7.80 \mathrm{E}-03$ & $1.07 \mathrm{E}-06$ & $6.10 \mathrm{E}-03$ & $-4.01 \mathrm{E}-04$ & $6.50 \mathrm{E}-03$ & $-4.62 \mathrm{E}-05$ & $1.17 \mathrm{E}+00$ \\
\hline 10000 & 174.8 & $7.78 \mathrm{E}-03$ & $1.27 \mathrm{E}-06$ & $6.09 \mathrm{E}-03$ & $-4.01 \mathrm{E}-04$ & $6.49 \mathrm{E}-03$ & $-4.62 \mathrm{E}-05$ & $1.13 \mathrm{E}+00$ \\
\hline 10000 & 169.8 & $7.76 \mathrm{E}-03$ & $1.91 \mathrm{E}-06$ & $6.07 \mathrm{E}-03$ & $-4.01 \mathrm{E}-04$ & $6.47 \mathrm{E}-03$ & $-4.66 \mathrm{E}-05$ & $1.10 \mathrm{E}+00$ \\
\hline 10000 & 164.8 & $7.74 \mathrm{E}-03$ & $1.06 \mathrm{E}-06$ & $6.06 \mathrm{E}-03$ & $-4.01 \mathrm{E}-04$ & $6.46 \mathrm{E}-03$ & $-4.58 \mathrm{E}-05$ & $1.06 \mathrm{E}+00$ \\
\hline 10000 & 159.8 & 7.72E-03 & $1.22 \mathrm{E}-06$ & $6.04 \mathrm{E}-03$ & $-4.01 \mathrm{E}-04$ & $6.44 \mathrm{E}-03$ & $-4.59 \mathrm{E}-05$ & $1.03 \mathrm{E}+00$ \\
\hline 10000 & 154.8 & 7.70E-03 & $2.12 \mathrm{E}-06$ & $6.03 \mathrm{E}-03$ & $-4.01 \mathrm{E}-04$ & $6.43 \mathrm{E}-03$ & $-4.64 \mathrm{E}-05$ & $9.95 \mathrm{E}-01$ \\
\hline 10000 & 149.9 & $7.68 \mathrm{E}-03$ & $1.58 \mathrm{E}-06$ & $6.01 \mathrm{E}-03$ & $-4.01 \mathrm{E}-04$ & $6.41 \mathrm{E}-03$ & $-4.59 \mathrm{E}-05$ & $9.61 \mathrm{E}-01$ \\
\hline 10000 & 144.9 & $7.66 \mathrm{E}-03$ & $2.10 \mathrm{E}-06$ & $5.99 \mathrm{E}-03$ & $-4.01 \mathrm{E}-04$ & $6.39 \mathrm{E}-03$ & $-4.62 \mathrm{E}-05$ & $9.26 \mathrm{E}-01$ \\
\hline 10000 & 139.9 & 7.63E-03 & $1.57 \mathrm{E}-06$ & $5.97 \mathrm{E}-03$ & $-4.01 \mathrm{E}-04$ & $6.37 \mathrm{E}-03$ & $-4.56 \mathrm{E}-05$ & 8.92E-01 \\
\hline 10000 & 134.9 & $7.61 \mathrm{E}-03$ & $1.76 \mathrm{E}-06$ & $5.95 \mathrm{E}-03$ & $-4.01 \mathrm{E}-04$ & $6.35 \mathrm{E}-03$ & $-4.56 \mathrm{E}-05$ & $8.57 \mathrm{E}-01$ \\
\hline 10000 & 129.9 & $7.58 \mathrm{E}-03$ & $2.25 \mathrm{E}-06$ & $5.93 \mathrm{E}-03$ & $-4.01 \mathrm{E}-04$ & $6.33 \mathrm{E}-03$ & $-4.58 \mathrm{E}-05$ & 8.23E-01 \\
\hline 10000 & 124.9 & 7.55E-03 & $2.14 \mathrm{E}-06$ & $5.91 \mathrm{E}-03$ & $-4.01 \mathrm{E}-04$ & $6.31 \mathrm{E}-03$ & $-4.56 \mathrm{E}-05$ & $7.88 \mathrm{E}-01$ \\
\hline 10000 & 119.9 & $7.52 \mathrm{E}-03$ & $2.94 \mathrm{E}-06$ & $5.88 \mathrm{E}-03$ & $-4.01 \mathrm{E}-04$ & $6.28 \mathrm{E}-03$ & $-4.60 \mathrm{E}-05$ & $7.54 \mathrm{E}-01$ \\
\hline 10000 & 115.0 & $7.48 \mathrm{E}-03$ & $3.22 \mathrm{E}-06$ & $5.86 \mathrm{E}-03$ & $-4.01 \mathrm{E}-04$ & $6.26 \mathrm{E}-03$ & $-4.61 \mathrm{E}-05$ & 7.19E-01 \\
\hline 10000 & 110.0 & $7.45 \mathrm{E}-03$ & $2.55 \mathrm{E}-06$ & $5.83 \mathrm{E}-03$ & $-4.01 \mathrm{E}-04$ & $6.23 \mathrm{E}-03$ & $-4.53 \mathrm{E}-05$ & $6.85 \mathrm{E}-$ \\
\hline
\end{tabular}




\begin{tabular}{|c|c|c|c|c|c|c|c|c|}
\hline 10000 & 105.0 & 7.40E-03 & $3.18 \mathrm{E}-06$ & 5.79E-03 & $-4.01 \mathrm{E}-04$ & $6.19 \mathrm{E}-03$ & $-4.55 \mathrm{E}-05$ & $6.50 \mathrm{E}-01$ \\
\hline 10000 & 100.0 & 7.35E-03 & $3.17 \mathrm{E}-06$ & $5.75 \mathrm{E}-03$ & $-4.01 \mathrm{E}-04$ & $6.15 \mathrm{E}-03$ & $-4.52 \mathrm{E}-05$ & $6.15 \mathrm{E}-01$ \\
\hline 10000 & 98.9 & 7.35E-03 & $9.96 \mathrm{E}-07$ & $5.75 \mathrm{E}-03$ & $-4.01 \mathrm{E}-04$ & $6.15 \mathrm{E}-03$ & $-4.35 \mathrm{E}-05$ & $6.08 \mathrm{E}-01$ \\
\hline 10000 & 97.9 & 7.33E-03 & $2.34 \mathrm{E}-06$ & $5.74 \mathrm{E}-03$ & $-4.01 \mathrm{E}-04$ & $6.14 \mathrm{E}-03$ & $-4.45 \mathrm{E}-05$ & $6.01 \mathrm{E}-01$ \\
\hline 10000 & 96.9 & 7.32E-03 & $1.79 \mathrm{E}-06$ & 5.73E-03 & $-4.01 \mathrm{E}-04$ & $6.13 \mathrm{E}-03$ & $-4.40 \mathrm{E}-05$ & $5.94 \mathrm{E}-01$ \\
\hline 10000 & 95.9 & 7.31E-03 & $1.64 \mathrm{E}-06$ & 5.72E-03 & $-4.01 \mathrm{E}-04$ & $6.12 \mathrm{E}-03$ & $-4.38 \mathrm{E}-05$ & $5.87 \mathrm{E}-01$ \\
\hline 10000 & 94.9 & $7.30 \mathrm{E}-03$ & $1.81 \mathrm{E}-06$ & $5.71 \mathrm{E}-03$ & $-4.01 \mathrm{E}-04$ & $6.11 \mathrm{E}-03$ & $-4.39 \mathrm{E}-05$ & $5.80 \mathrm{E}-01$ \\
\hline 10000 & 93.9 & 7.29E-03 & $2.00 \mathrm{E}-06$ & $5.70 \mathrm{E}-03$ & $-4.01 \mathrm{E}-04$ & $6.10 \mathrm{E}-03$ & $-4.39 \mathrm{E}-05$ & 5.73E-01 \\
\hline 10000 & 92.9 & $7.28 \mathrm{E}-03$ & $1.99 \mathrm{E}-06$ & $5.69 \mathrm{E}-03$ & $-4.01 \mathrm{E}-04$ & $6.09 \mathrm{E}-03$ & $-4.39 \mathrm{E}-05$ & $5.66 \mathrm{E}-01$ \\
\hline 10000 & 91.9 & $7.26 \mathrm{E}-03$ & $2.50 \mathrm{E}-06$ & $5.68 \mathrm{E}-03$ & $-4.01 \mathrm{E}-04$ & $6.08 \mathrm{E}-03$ & $-4.42 \mathrm{E}-05$ & $5.59 \mathrm{E}-01$ \\
\hline 10000 & 90.9 & $7.25 \mathrm{E}-03$ & $2.14 \mathrm{E}-06$ & $5.67 \mathrm{E}-03$ & $-4.01 \mathrm{E}-04$ & $6.07 \mathrm{E}-03$ & $-4.38 \mathrm{E}-05$ & $5.52 \mathrm{E}-01$ \\
\hline 10000 & 89.9 & 7.23E-03 & $2.31 \mathrm{E}-06$ & $5.66 \mathrm{E}-03$ & $-4.01 \mathrm{E}-04$ & $6.06 \mathrm{E}-03$ & $-4.39 \mathrm{E}-05$ & $5.45 \mathrm{E}-01$ \\
\hline 10000 & 88.9 & 7.22E-03 & $2.10 \mathrm{E}-06$ & $5.65 \mathrm{E}-03$ & $-4.01 \mathrm{E}-04$ & $6.05 \mathrm{E}-03$ & $-4.36 \mathrm{E}-05$ & $5.38 \mathrm{E}-01$ \\
\hline 10000 & 87.9 & $7.20 \mathrm{E}-03$ & $2.29 \mathrm{E}-06$ & $5.64 \mathrm{E}-03$ & $-4.01 \mathrm{E}-04$ & $6.04 \mathrm{E}-03$ & $-4.37 \mathrm{E}-05$ & $5.31 \mathrm{E}-01$ \\
\hline 10000 & 86.9 & 7.19E-03 & $2.34 \mathrm{E}-06$ & $5.62 \mathrm{E}-03$ & $-4.01 \mathrm{E}-04$ & $6.02 \mathrm{E}-03$ & $-4.36 \mathrm{E}-05$ & $5.24 \mathrm{E}-01$ \\
\hline 10000 & 85.9 & 7.17E-03 & $2.77 \mathrm{E}-06$ & $5.61 \mathrm{E}-03$ & $-4.01 \mathrm{E}-04$ & $6.01 \mathrm{E}-03$ & $-4.39 \mathrm{E}-05$ & $5.16 \mathrm{E}-01$ \\
\hline 10000 & 84.9 & 7.15E-03 & $2.48 \mathrm{E}-06$ & $5.60 \mathrm{E}-03$ & $-4.01 \mathrm{E}-04$ & $6.00 \mathrm{E}-03$ & $-4.35 \mathrm{E}-05$ & $5.09 \mathrm{E}-01$ \\
\hline 10000 & 83.9 & 7.13E-03 & $2.66 \mathrm{E}-06$ & $5.58 \mathrm{E}-03$ & $-4.01 \mathrm{E}-04$ & $5.98 \mathrm{E}-03$ & $-4.36 \mathrm{E}-05$ & $5.02 \mathrm{E}-01$ \\
\hline 10000 & 82.9 & 7.11E-03 & $2.55 \mathrm{E}-06$ & $5.57 \mathrm{E}-03$ & $-4.01 \mathrm{E}-04$ & 5.97E-03 & $-4.34 \mathrm{E}-05$ & 4.95E-01 \\
\hline 10000 & 81.9 & 7.09E-03 & $3.11 \mathrm{E}-06$ & $5.55 \mathrm{E}-03$ & $-4.01 \mathrm{E}-04$ & $5.95 \mathrm{E}-03$ & $-4.37 \mathrm{E}-05$ & $4.88 \mathrm{E}-01$ \\
\hline 10000 & 80.9 & 7.07E-03 & $3.36 \mathrm{E}-06$ & $5.54 \mathrm{E}-03$ & $-4.01 \mathrm{E}-04$ & $5.94 \mathrm{E}-03$ & $-4.38 \mathrm{E}-05$ & $4.81 \mathrm{E}-01$ \\
\hline 10000 & 80.0 & 7.05E-03 & $3.39 \mathrm{E}-06$ & $5.52 \mathrm{E}-03$ & $-4.01 \mathrm{E}-04$ & $5.92 \mathrm{E}-03$ & $-4.37 \mathrm{E}-05$ & 4.73E-01 \\
\hline 10000 & 79.0 & 7.03E-03 & $3.52 \mathrm{E}-06$ & $5.50 \mathrm{E}-03$ & $-4.01 \mathrm{E}-04$ & $5.90 \mathrm{E}-03$ & $-4.37 \mathrm{E}-05$ & 4.66E-01 \\
\hline 10000 & 78.0 & 7.01E-03 & $3.48 \mathrm{E}-06$ & $5.48 \mathrm{E}-03$ & $-4.01 \mathrm{E}-04$ & $5.89 \mathrm{E}-03$ & $-4.35 \mathrm{E}-05$ & $4.59 \mathrm{E}-01$ \\
\hline 10000 & 77.0 & $6.99 \mathrm{E}-03$ & $4.04 \mathrm{E}-06$ & $5.47 \mathrm{E}-03$ & $-4.01 \mathrm{E}-04$ & $5.87 \mathrm{E}-03$ & $-4.38 \mathrm{E}-05$ & $4.51 \mathrm{E}-01$ \\
\hline 10000 & 76.0 & $6.96 \mathrm{E}-03$ & $3.98 \mathrm{E}-06$ & $5.45 \mathrm{E}-03$ & $-4.01 \mathrm{E}-04$ & $5.85 \mathrm{E}-03$ & $-4.36 \mathrm{E}-05$ & 4.44E-01 \\
\hline 10000 & 75.0 & $6.94 \mathrm{E}-03$ & $3.84 \mathrm{E}-06$ & $5.43 \mathrm{E}-03$ & $-4.01 \mathrm{E}-04$ & $5.83 \mathrm{E}-03$ & $-4.33 \mathrm{E}-05$ & 4.37E-01 \\
\hline 10000 & 74.0 & $6.91 \mathrm{E}-03$ & $4.26 \mathrm{E}-06$ & $5.41 \mathrm{E}-03$ & $-4.01 \mathrm{E}-04$ & $5.81 \mathrm{E}-03$ & $-4.35 E-05$ & 4.30E-01 \\
\hline 10000 & 73.0 & $6.88 \mathrm{E}-03$ & 4.04E-06 & $5.38 \mathrm{E}-03$ & $-4.01 \mathrm{E}-04$ & $5.79 \mathrm{E}-03$ & $-4.32 \mathrm{E}-05$ & 4.22E-01 \\
\hline 10000 & 72.0 & $6.85 \mathrm{E}-03$ & $4.36 \mathrm{E}-06$ & $5.36 \mathrm{E}-03$ & $-4.01 \mathrm{E}-04$ & $5.76 \mathrm{E}-03$ & $-4.33 \mathrm{E}-05$ & $4.15 \mathrm{E}-01$ \\
\hline 10000 & 71.0 & $6.82 \mathrm{E}-03$ & $4.35 \mathrm{E}-06$ & $5.34 \mathrm{E}-03$ & $-4.01 \mathrm{E}-04$ & $5.74 \mathrm{E}-03$ & $-4.31 \mathrm{E}-05$ & $4.08 \mathrm{E}-01$ \\
\hline 10000 & 70.0 & $6.79 \mathrm{E}-03$ & $4.52 \mathrm{E}-06$ & $5.32 \mathrm{E}-03$ & $-4.01 \mathrm{E}-04$ & $5.72 \mathrm{E}-03$ & $-4.31 \mathrm{E}-05$ & $4.00 \mathrm{E}-01$ \\
\hline 10000 & 69.0 & $6.76 \mathrm{E}-03$ & $4.40 \mathrm{E}-06$ & $5.29 \mathrm{E}-03$ & $-4.01 \mathrm{E}-04$ & $5.69 \mathrm{E}-03$ & $-4.28 \mathrm{E}-05$ & $3.93 \mathrm{E}-01$ \\
\hline 10000 & 68.0 & $6.73 \mathrm{E}-03$ & $4.50 \mathrm{E}-06$ & $5.26 \mathrm{E}-03$ & $-4.01 \mathrm{E}-04$ & $5.67 \mathrm{E}-03$ & $-4.27 \mathrm{E}-05$ & $3.85 \mathrm{E}-01$ \\
\hline 10000 & 67.0 & $6.69 \mathrm{E}-03$ & 4.64E-06 & $5.24 \mathrm{E}-03$ & $-4.01 \mathrm{E}-04$ & $5.64 \mathrm{E}-03$ & $-4.26 \mathrm{E}-05$ & $3.78 \mathrm{E}-01$ \\
\hline 10000 & 66.0 & $6.66 \mathrm{E}-03$ & $5.58 \mathrm{E}-07$ & $5.21 \mathrm{E}-03$ & $-4.01 \mathrm{E}-04$ & $5.61 \mathrm{E}-03$ & $-3.92 \mathrm{E}-05$ & $3.71 \mathrm{E}-01$ \\
\hline 10000 & 65.0 & $6.63 \mathrm{E}-03$ & $1.64 \mathrm{E}-07$ & $5.19 \mathrm{E}-03$ & $-4.01 \mathrm{E}-04$ & $5.59 \mathrm{E}-03$ & $-3.87 \mathrm{E}-05$ & $3.63 \mathrm{E}-01$ \\
\hline 10000 & 64.0 & $6.59 \mathrm{E}-03$ & $3.95 \mathrm{E}-07$ & $5.16 \mathrm{E}-03$ & $-4.01 \mathrm{E}-04$ & $5.56 \mathrm{E}-03$ & $-3.86 \mathrm{E}-05$ & $3.56 \mathrm{E}-01$ \\
\hline 10000 & 63.0 & $6.55 \mathrm{E}-03$ & $9.29 \mathrm{E}-07$ & $5.12 \mathrm{E}-03$ & $-4.01 \mathrm{E}-04$ & $5.53 \mathrm{E}-03$ & $-3.88 \mathrm{E}-05$ & $3.48 \mathrm{E}-01$ \\
\hline 10000 & 62.0 & $6.51 \mathrm{E}-03$ & $1.91 \mathrm{E}-07$ & $5.09 \mathrm{E}-03$ & $-4.01 \mathrm{E}-04$ & $5.49 \mathrm{E}-03$ & $-3.80 \mathrm{E}-05$ & $3.41 \mathrm{E}-01$ \\
\hline 10000 & 61.0 & $6.47 \mathrm{E}-03$ & 1.69E-07 & $5.06 \mathrm{E}-03$ & $-4.01 \mathrm{E}-04$ & $5.46 \mathrm{E}-03$ & $-3.77 \mathrm{E}-05$ & $3.33 \mathrm{E}-01$ \\
\hline 10000 & 60.0 & $6.42 \mathrm{E}-03$ & $3.37 \mathrm{E}-06$ & $5.02 \mathrm{E}-03$ & $-4.01 \mathrm{E}-04$ & $5.42 \mathrm{E}-03$ & $-4.00 \mathrm{E}-05$ & 3.26E-01 \\
\hline 10000 & 59.0 & $6.38 \mathrm{E}-03$ & $6.28 \mathrm{E}-07$ & 4.99E-03 & $-4.01 \mathrm{E}-04$ & 5.39E-03 & $-3.76 \mathrm{E}-05$ & $3.18 \mathrm{E}-01$ \\
\hline
\end{tabular}




\begin{tabular}{|c|c|c|c|c|c|c|c|c|}
\hline 10000 & 58.0 & $6.33 \mathrm{E}-03$ & $7.52 \mathrm{E}-07$ & $4.95 \mathrm{E}-03$ & $-4.01 \mathrm{E}-04$ & $5.35 \mathrm{E}-03$ & $-3.74 \mathrm{E}-05$ & $3.11 \mathrm{E}-01$ \\
\hline 10000 & 57.0 & $6.28 \mathrm{E}-03$ & $3.46 \mathrm{E}-07$ & $4.92 \mathrm{E}-03$ & $-4.01 \mathrm{E}-04$ & $5.32 \mathrm{E}-03$ & $-3.68 \mathrm{E}-05$ & $3.03 \mathrm{E}-01$ \\
\hline 10000 & 56.0 & $6.23 \mathrm{E}-03$ & 3.32E-07 & $4.88 \mathrm{E}-03$ & $-4.01 \mathrm{E}-04$ & $5.28 \mathrm{E}-03$ & $-3.65 \mathrm{E}-05$ & $2.96 \mathrm{E}-01$ \\
\hline 10000 & 55.0 & $6.18 \mathrm{E}-03$ & $2.16 \mathrm{E}-07$ & $4.84 \mathrm{E}-03$ & $-4.01 \mathrm{E}-04$ & $5.24 \mathrm{E}-03$ & $-3.61 \mathrm{E}-05$ & $2.88 \mathrm{E}-01$ \\
\hline 10000 & 54.0 & $6.13 \mathrm{E}-03$ & $2.16 \mathrm{E}-07$ & $4.80 \mathrm{E}-03$ & $-4.01 \mathrm{E}-04$ & $5.20 \mathrm{E}-03$ & $-3.58 \mathrm{E}-05$ & $2.81 \mathrm{E}-01$ \\
\hline 10000 & 53.1 & $6.08 \mathrm{E}-03$ & $1.23 \mathrm{E}-06$ & $4.76 \mathrm{E}-03$ & $-4.01 \mathrm{E}-04$ & $5.16 \mathrm{E}-03$ & $-3.63 \mathrm{E}-05$ & $2.74 \mathrm{E}-01$ \\
\hline 10000 & 52.0 & $6.02 \mathrm{E}-03$ & $5.29 \mathrm{E}-08$ & $4.71 \mathrm{E}-03$ & $-4.01 \mathrm{E}-04$ & $5.11 \mathrm{E}-03$ & $-3.51 \mathrm{E}-05$ & $2.66 \mathrm{E}-01$ \\
\hline 10000 & 51.0 & $5.97 \mathrm{E}-03$ & $1.17 \mathrm{E}-06$ & 4.67E-03 & $-4.01 \mathrm{E}-04$ & 5.07E-03 & $-3.56 \mathrm{E}-05$ & $2.59 \mathrm{E}-01$ \\
\hline 10000 & 50.0 & $5.91 \mathrm{E}-03$ & $1.22 \mathrm{E}-06$ & 4.63E-03 & $-4.01 \mathrm{E}-04$ & $5.03 \mathrm{E}-03$ & $-3.53 \mathrm{E}-05$ & $2.52 \mathrm{E}-01$ \\
\hline 10000 & 49.0 & $5.85 \mathrm{E}-03$ & 4.79E-07 & $4.58 \mathrm{E}-03$ & $-4.01 \mathrm{E}-04$ & $4.98 \mathrm{E}-03$ & $-3.44 \mathrm{E}-05$ & $2.44 \mathrm{E}-01$ \\
\hline 10000 & 48.0 & $5.80 \mathrm{E}-03$ & $6.63 \mathrm{E}-07$ & $4.54 \mathrm{E}-03$ & $-4.01 \mathrm{E}-04$ & 4.94E-03 & $-3.42 \mathrm{E}-05$ & $2.37 \mathrm{E}-01$ \\
\hline 10000 & 47.0 & $5.74 \mathrm{E}-03$ & $4.14 \mathrm{E}-07$ & 4.49E-03 & $-4.01 \mathrm{E}-04$ & $4.89 \mathrm{E}-03$ & $-3.37 \mathrm{E}-05$ & $2.30 \mathrm{E}-01$ \\
\hline 10000 & 46.0 & $5.68 \mathrm{E}-03$ & $3.60 \mathrm{E}-07$ & $4.45 \mathrm{E}-03$ & $-4.01 \mathrm{E}-04$ & $4.85 \mathrm{E}-03$ & $-3.33 \mathrm{E}-05$ & $2.23 \mathrm{E}-01$ \\
\hline 10000 & 45.0 & $5.63 \mathrm{E}-03$ & $5.08 \mathrm{E}-07$ & $4.40 \mathrm{E}-03$ & $-4.01 \mathrm{E}-04$ & $4.80 \mathrm{E}-03$ & $-3.31 \mathrm{E}-05$ & $2.16 \mathrm{E}-01$ \\
\hline 10000 & 44.0 & $5.57 \mathrm{E}-03$ & 5.61E-07 & $4.36 \mathrm{E}-03$ & $-4.01 \mathrm{E}-04$ & $4.76 \mathrm{E}-03$ & $-3.28 \mathrm{E}-05$ & $2.10 \mathrm{E}-01$ \\
\hline 10000 & 43.0 & $5.52 \mathrm{E}-03$ & 4.94E-07 & $4.32 \mathrm{E}-03$ & $-4.01 \mathrm{E}-04$ & 4.72E-03 & $-3.25 \mathrm{E}-05$ & $2.03 \mathrm{E}-01$ \\
\hline 10000 & 42.0 & $5.46 \mathrm{E}-03$ & 4.04E-07 & 4.27E-03 & $-4.01 \mathrm{E}-04$ & $4.68 \mathrm{E}-03$ & $-3.21 \mathrm{E}-05$ & $1.96 \mathrm{E}-01$ \\
\hline 10000 & 41.0 & $5.41 \mathrm{E}-03$ & $3.49 \mathrm{E}-07$ & $4.23 \mathrm{E}-03$ & $-4.01 \mathrm{E}-04$ & $4.63 \mathrm{E}-03$ & $-3.17 \mathrm{E}-05$ & $1.90 \mathrm{E}-01$ \\
\hline 10000 & 40.0 & $5.36 \mathrm{E}-03$ & $5.85 \mathrm{E}-07$ & $4.20 \mathrm{E}-03$ & $-4.01 \mathrm{E}-04$ & $4.60 \mathrm{E}-03$ & $-3.16 \mathrm{E}-05$ & $1.84 \mathrm{E}-01$ \\
\hline 10000 & 39.0 & 5.32E-03 & $1.98 \mathrm{E}-07$ & $4.16 \mathrm{E}-03$ & $-4.01 \mathrm{E}-04$ & $4.56 \mathrm{E}-03$ & $-3.11 \mathrm{E}-05$ & $1.78 \mathrm{E}-01$ \\
\hline 10000 & 38.0 & $5.28 \mathrm{E}-03$ & $2.36 \mathrm{E}-07$ & $4.13 \mathrm{E}-03$ & $-4.01 \mathrm{E}-04$ & $4.53 \mathrm{E}-03$ & $-3.09 \mathrm{E}-05$ & $1.72 \mathrm{E}-01$ \\
\hline 10000 & 37.0 & $5.24 \mathrm{E}-03$ & $6.53 \mathrm{E}-07$ & $4.10 \mathrm{E}-03$ & $-4.01 \mathrm{E}-04$ & $4.50 \mathrm{E}-03$ & $-3.10 \mathrm{E}-05$ & $1.67 \mathrm{E}-01$ \\
\hline 10000 & 36.0 & $5.21 \mathrm{E}-03$ & $1.95 \mathrm{E}-07$ & $4.08 \mathrm{E}-03$ & $-4.01 \mathrm{E}-04$ & $4.48 \mathrm{E}-03$ & $-3.04 \mathrm{E}-05$ & $1.61 \mathrm{E}-01$ \\
\hline 10000 & 35.0 & $5.18 \mathrm{E}-03$ & $3.95 \mathrm{E}-07$ & $4.06 \mathrm{E}-03$ & $-4.01 \mathrm{E}-04$ & $4.46 \mathrm{E}-03$ & $-3.04 \mathrm{E}-05$ & $1.56 \mathrm{E}-01$ \\
\hline 10000 & 34.0 & $5.16 \mathrm{E}-03$ & $4.13 \mathrm{E}-07$ & $4.04 \mathrm{E}-03$ & $-4.01 \mathrm{E}-04$ & 4.44E-03 & $-3.03 \mathrm{E}-05$ & $1.51 \mathrm{E}-01$ \\
\hline 10000 & 33.0 & $5.15 \mathrm{E}-03$ & $3.54 \mathrm{E}-07$ & 4.03E-03 & $-4.01 \mathrm{E}-04$ & 4.43E-03 & $-3.02 \mathrm{E}-05$ & $1.46 \mathrm{E}-01$ \\
\hline 10000 & 32.0 & $5.15 \mathrm{E}-03$ & $4.38 \mathrm{E}-07$ & $4.03 \mathrm{E}-03$ & $-4.01 \mathrm{E}-04$ & 4.43E-03 & $-3.03 \mathrm{E}-05$ & $1.42 \mathrm{E}-01$ \\
\hline 10000 & 31.0 & $5.16 \mathrm{E}-03$ & $1.93 \mathrm{E}-07$ & $4.04 \mathrm{E}-03$ & $-4.01 \mathrm{E}-04$ & 4.44E-03 & $-3.01 \mathrm{E}-05$ & $1.38 \mathrm{E}-01$ \\
\hline 10000 & 30.0 & $5.18 \mathrm{E}-03$ & $2.01 \mathrm{E}-07$ & $4.05 \mathrm{E}-03$ & $-4.01 \mathrm{E}-04$ & $4.45 \mathrm{E}-03$ & $-3.03 \mathrm{E}-05$ & $1.34 \mathrm{E}-01$ \\
\hline 10000 & 29.0 & $5.21 \mathrm{E}-03$ & $3.22 \mathrm{E}-07$ & 4.07E-03 & $-4.01 \mathrm{E}-04$ & $4.48 \mathrm{E}-03$ & $-3.05 \mathrm{E}-05$ & $1.30 \mathrm{E}-01$ \\
\hline 10000 & 28.0 & $5.25 \mathrm{E}-03$ & $2.58 \mathrm{E}-07$ & $4.11 \mathrm{E}-03$ & $-4.01 \mathrm{E}-04$ & $4.51 \mathrm{E}-03$ & $-3.07 \mathrm{E}-05$ & $1.26 \mathrm{E}-01$ \\
\hline 10000 & 27.0 & $5.31 \mathrm{E}-03$ & $1.47 \mathrm{E}-07$ & $4.15 \mathrm{E}-03$ & $-4.01 \mathrm{E}-04$ & 4.55E-03 & $-3.10 \mathrm{E}-05$ & $1.23 \mathrm{E}-01$ \\
\hline 10000 & 26.0 & $5.38 \mathrm{E}-03$ & $2.34 \mathrm{E}-07$ & $4.21 \mathrm{E}-03$ & $-4.01 \mathrm{E}-04$ & 4.61E-03 & $-3.15 \mathrm{E}-05$ & $1.20 \mathrm{E}-01$ \\
\hline 10000 & 25.0 & $5.47 \mathrm{E}-03$ & $9.56 \mathrm{E}-07$ & $4.28 \mathrm{E}-03$ & $-4.01 \mathrm{E}-04$ & $4.68 \mathrm{E}-03$ & $-3.25 \mathrm{E}-05$ & $1.17 \mathrm{E}-01$ \\
\hline 10000 & 24.0 & $5.57 \mathrm{E}-03$ & $1.62 \mathrm{E}-06$ & $4.36 \mathrm{E}-03$ & $-4.01 \mathrm{E}-04$ & $4.76 \mathrm{E}-03$ & $-3.37 \mathrm{E}-05$ & $1.14 \mathrm{E}-01$ \\
\hline 10000 & 23.0 & $5.70 \mathrm{E}-03$ & 7.43E-07 & $4.46 \mathrm{E}-03$ & $-4.01 \mathrm{E}-04$ & 4.86E-03 & $-3.37 \mathrm{E}-05$ & $1.12 \mathrm{E}-01$ \\
\hline 10000 & 22.0 & $5.84 \mathrm{E}-03$ & 4.38E-07 & 4.57E-03 & $-4.01 \mathrm{E}-04$ & 4.97E-03 & $-3.43 \mathrm{E}-05$ & $1.09 \mathrm{E}-01$ \\
\hline 10000 & 21.0 & $6.01 \mathrm{E}-03$ & $2.51 \mathrm{E}-07$ & $4.70 \mathrm{E}-03$ & $-4.01 \mathrm{E}-04$ & $5.10 \mathrm{E}-03$ & $-3.51 \mathrm{E}-05$ & $1.07 \mathrm{E}-01$ \\
\hline 10000 & 20.0 & $6.20 \mathrm{E}-03$ & 4.17E-07 & $4.85 \mathrm{E}-03$ & $-4.01 \mathrm{E}-04$ & $5.25 \mathrm{E}-03$ & $-3.64 \mathrm{E}-05$ & $1.05 \mathrm{E}-01$ \\
\hline 10000 & 19.0 & $6.41 \mathrm{E}-03$ & $3.29 \mathrm{E}-07$ & $5.02 \mathrm{E}-03$ & $-4.01 \mathrm{E}-04$ & $5.42 \mathrm{E}-03$ & $-3.75 \mathrm{E}-05$ & $1.03 \mathrm{E}-01$ \\
\hline 10000 & 18.0 & $6.65 \mathrm{E}-03$ & $2.32 \mathrm{E}-06$ & $5.21 \mathrm{E}-03$ & $-4.01 \mathrm{E}-04$ & $5.61 \mathrm{E}-03$ & $-4.05 \mathrm{E}-05$ & $1.01 \mathrm{E}-01$ \\
\hline 10000 & 17.0 & $6.93 \mathrm{E}-03$ & $2.03 \mathrm{E}-06$ & $5.42 \mathrm{E}-03$ & $-4.01 \mathrm{E}-04$ & $5.82 \mathrm{E}-03$ & $-4.19 \mathrm{E}-05$ & $9.90 \mathrm{E}-02$ \\
\hline 10000 & 16.0 & 7.23E-03 & $1.18 \mathrm{E}-06$ & $5.66 \mathrm{E}-03$ & $-4.01 \mathrm{E}-04$ & $6.06 \mathrm{E}-03$ & $-4.30 \mathrm{E}-05$ & $9.70 \mathrm{E}-02$ \\
\hline
\end{tabular}




$\begin{array}{lllllllll}10000 & 15.0 & 7.58 \mathrm{E}-03 & 1.32 \mathrm{E}-06 & 5.93 \mathrm{E}-03 & -4.01 \mathrm{E}-04 & 6.33 \mathrm{E}-03 & -4.51 \mathrm{E}-05 & 9.50 \mathrm{E}-02 \\ 10000 & 14.0 & 7.97 \mathrm{E}-03 & 2.29 \mathrm{E}-06 & 6.23 \mathrm{E}-03 & -4.01 \mathrm{E}-04 & 6.63 \mathrm{E}-03 & -4.81 \mathrm{E}-05 & 9.29 \mathrm{E}-02 \\ 10000 & 13.0 & 8.40 \mathrm{E}-03 & 1.45 \mathrm{E}-06 & 6.58 \mathrm{E}-03 & -4.01 \mathrm{E}-04 & 6.98 \mathrm{E}-03 & -5.00 \mathrm{E}-05 & 9.07 \mathrm{E}-02 \\ 10000 & 12.0 & 8.91 \mathrm{E}-03 & 1.44 \mathrm{E}-05 & 6.97 \mathrm{E}-03 & -4.01 \mathrm{E}-04 & 7.37 \mathrm{E}-03 & -6.32 \mathrm{E}-05 & 8.85 \mathrm{E}-02 \\ 10000 & 11.0 & 9.49 \mathrm{E}-03 & 3.53 \mathrm{E}-06 & 7.43 \mathrm{E}-03 & -4.01 \mathrm{E}-04 & 7.83 \mathrm{E}-03 & -5.80 \mathrm{E}-05 & 8.62 \mathrm{E}-02 \\ 10000 & 10.0 & 1.02 \mathrm{E}-02 & 4.01 \mathrm{E}-06 & 7.96 \mathrm{E}-03 & -4.01 \mathrm{E}-04 & 8.36 \mathrm{E}-03 & -6.23 \mathrm{E}-05 & 8.37 \mathrm{E}-02 \\ 10000 & 9.0 & 1.10 \mathrm{E}-02 & 3.94 \mathrm{E}-06 & 8.60 \mathrm{E}-03 & -4.01 \mathrm{E}-04 & 9.00 \mathrm{E}-03 & -6.70 \mathrm{E}-05 & 8.10 \mathrm{E}-02 \\ 10000 & 8.0 & 1.20 \mathrm{E}-02 & 1.20 \mathrm{E}-05 & 9.36 \mathrm{E}-03 & -4.01 \mathrm{E}-04 & 9.76 \mathrm{E}-03 & -7.90 \mathrm{E}-05 & 7.82 \mathrm{E}-02 \\ 10000 & 7.0 & 1.32 \mathrm{E}-02 & 1.12 \mathrm{E}-05 & 1.03 \mathrm{E}-02 & -4.01 \mathrm{E}-04 & 1.07 \mathrm{E}-02 & -8.54 \mathrm{E}-05 & 7.50 \mathrm{E}-02 \\ 10000 & 6.0 & 1.47 \mathrm{E}-02 & 1.57 \mathrm{E}-05 & 1.15 \mathrm{E}-02 & -4.01 \mathrm{E}-04 & 1.19 \mathrm{E}-02 & -9.78 \mathrm{E}-05 & 7.15 \mathrm{E}-02 \\ 10000 & 5.0 & 1.67 \mathrm{E}-02 & 1.91 \mathrm{E}-05 & 1.30 \mathrm{E}-02 & -4.01 \mathrm{E}-04 & 1.34 \mathrm{E}-02 & -1.12 \mathrm{E}-04 & 6.73 \mathrm{E}-02\end{array}$


Figures S27-S28: SQUID plots for complexes 3 - 4
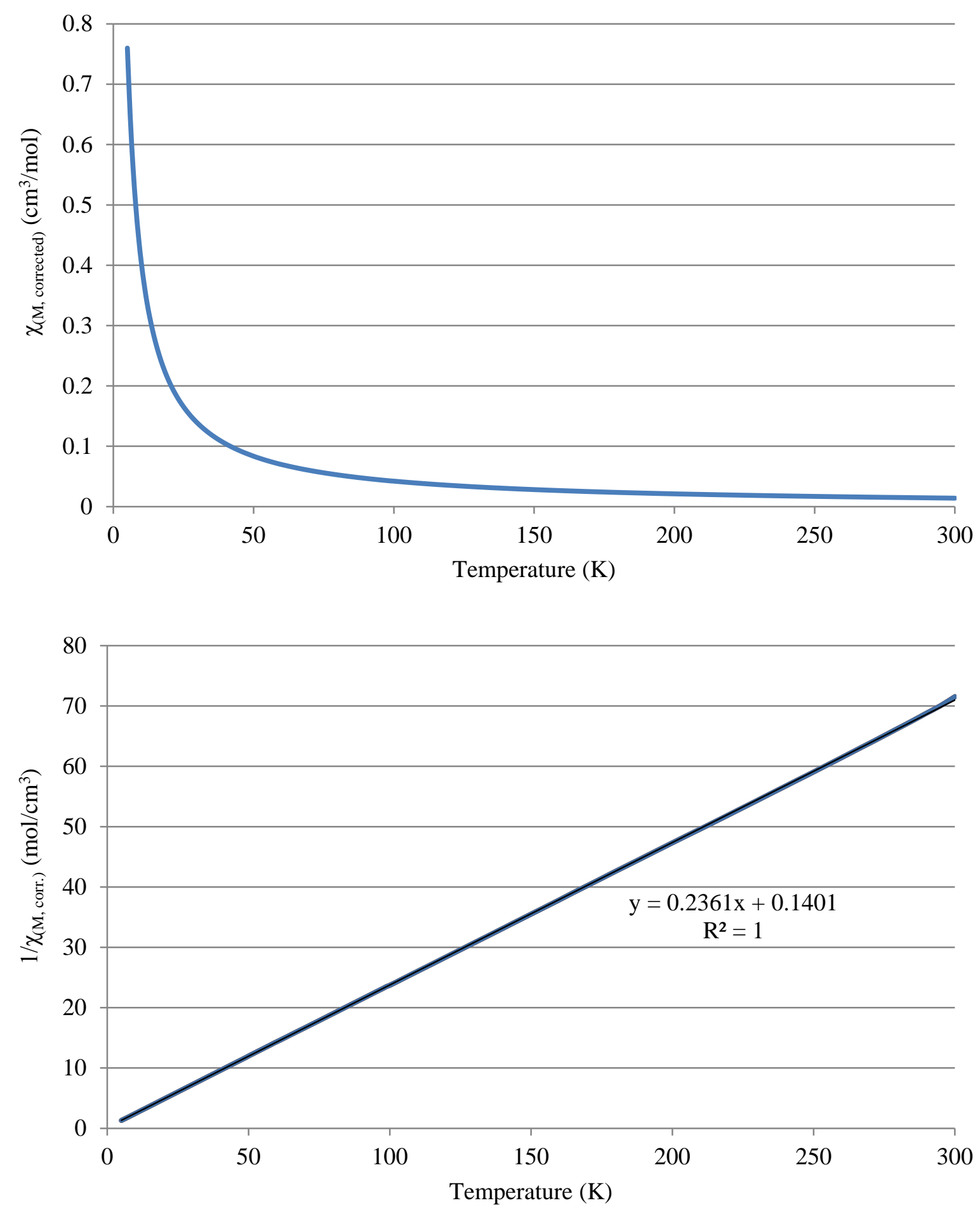

Figure S27: $\quad$ SQUID plots of $\left[\mathrm{Mn}\left(\mathrm{CH}_{2} \mathrm{SiMe}_{3}\right)_{2}(\mathrm{dmpe})\right](3) ; \chi_{(\mathrm{M}, \text { corrected })}$ vs. T (top) and $\chi_{(\mathrm{M}, \text { corrected) }}{ }^{-1}$ vs. $T$ (bottom). The blue line in the bottom graph represents the data and the black line superimposed above it is a linear line of best fit. Molar magnetic susceptibilities are calculated using an average of two attempts to measure the mass of sample. 

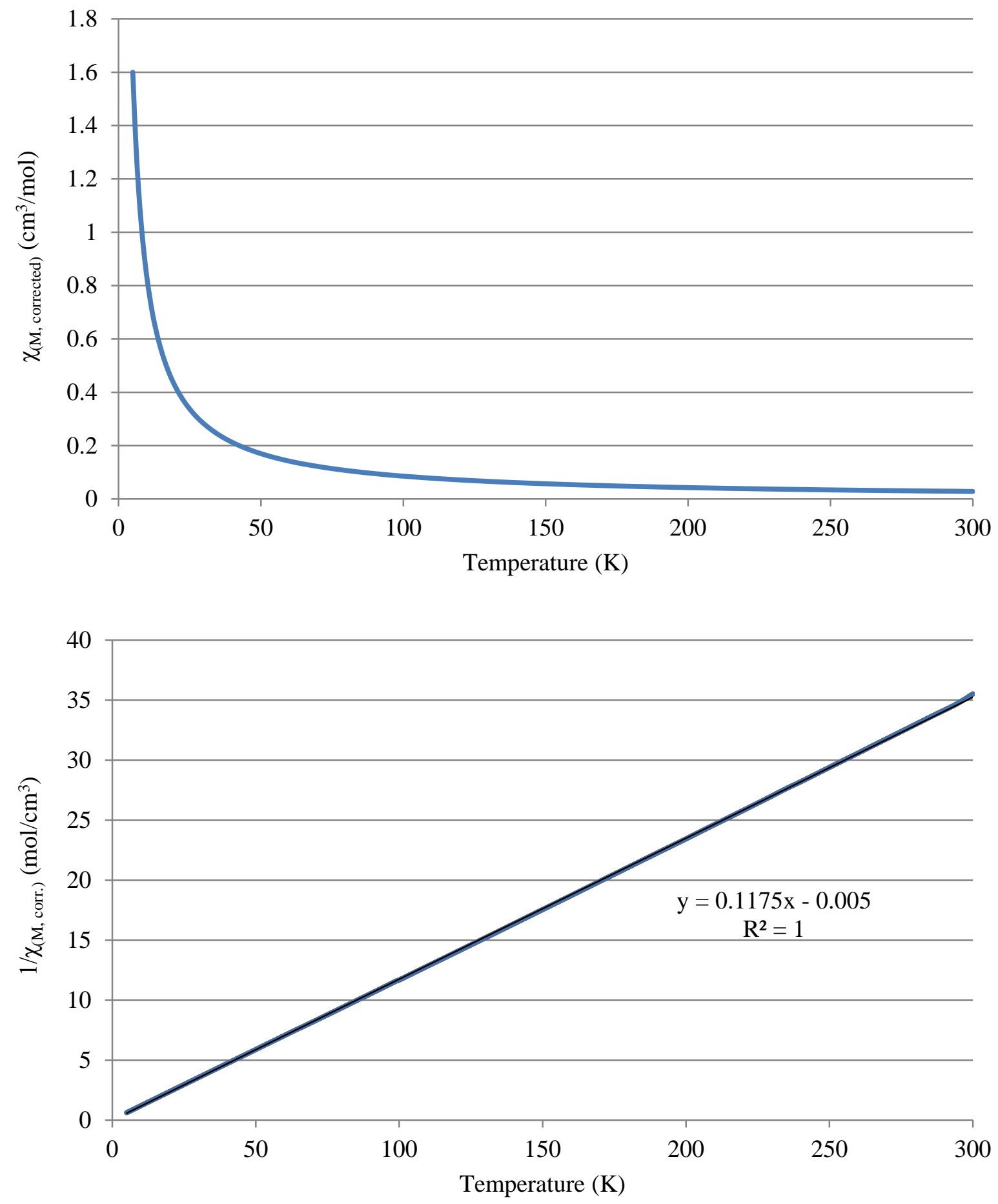

Figure S28: $\quad$ SQUID plots of $\left[\left\{\mathrm{Mn}\left(\mathrm{CH}_{2} \mathrm{CMe}_{3}\right)_{2}(\mu \text {-dmpe })\right\}_{2}\right](4) ; \chi_{(M, \text { corrected })}$ vs. T (top) and $\chi_{(\mathrm{M} \text {, }}$ corrected) $^{-1}$ vs. $T$ (bottom), and $\mu_{\text {eff }}$ vs. T (bottom). The blue line in the bottom graph represents the data and the black line superimposed above it is a linear line of best fit. 
Figures S29-S33: SQUID and fits to an exchange expression for 2 and 5 -8
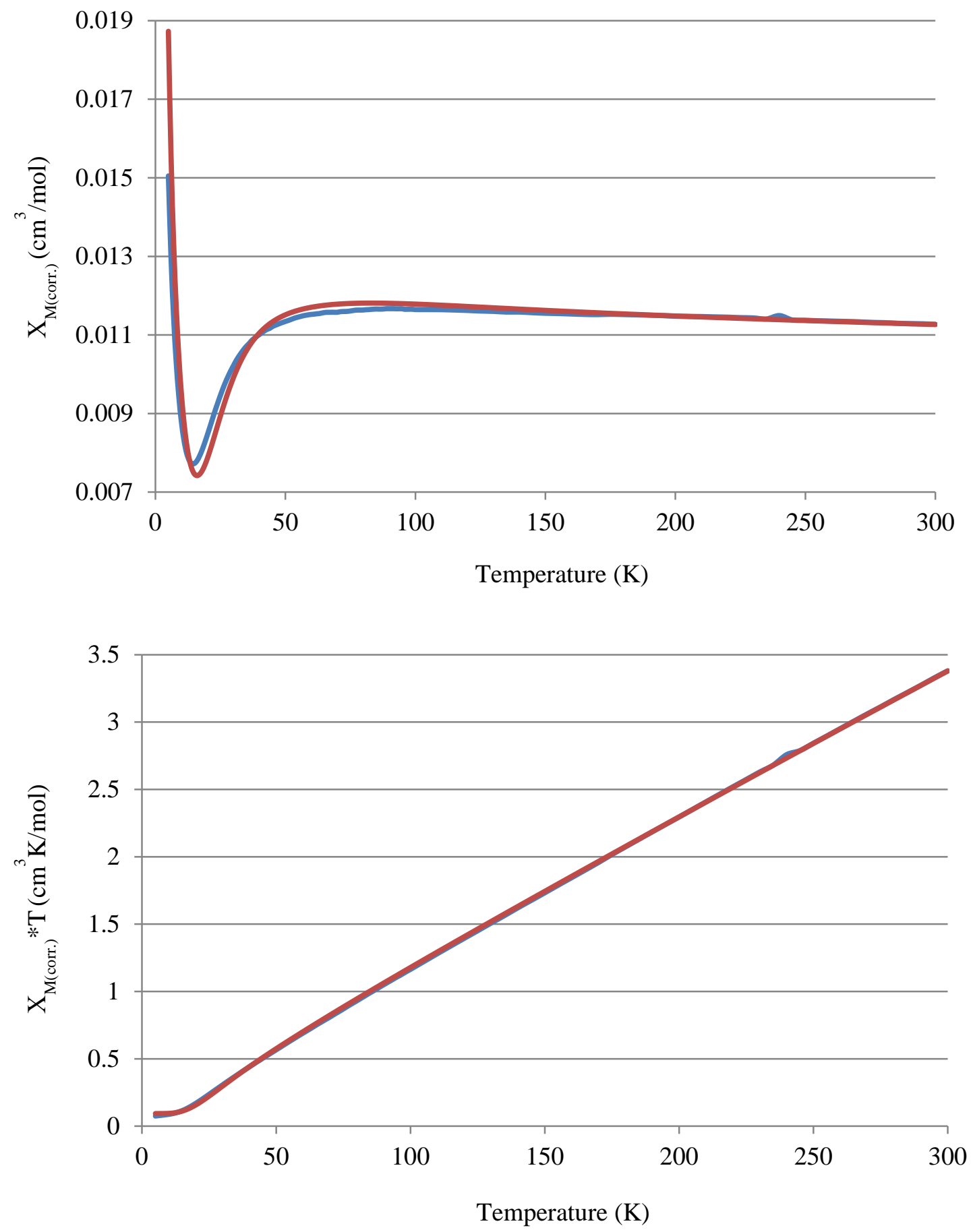

Figure S29: $\quad$ SQUID of $\left[\left\{\mathrm{Mn}\left(\mathrm{CH}_{2} \mathrm{CMe}_{3}\right)\left(\mu-\mathrm{CH}_{2} \mathrm{CMe}_{3}\right)_{2}\right\}_{2}\left\{\mathrm{Mn}\left(\mu-\mathrm{CH}_{2} \mathrm{CMe}_{3}\right)_{2} \mathrm{Mn}\right\}\right]$ (2) (blue) with fit to van Vleck equation ${ }^{[5]}$ (red) for $\chi_{(M, \text { corrected) }}$ vs. T (top) and $\chi_{(M, \text { corrected })} * T$ vs. T (bottom) 

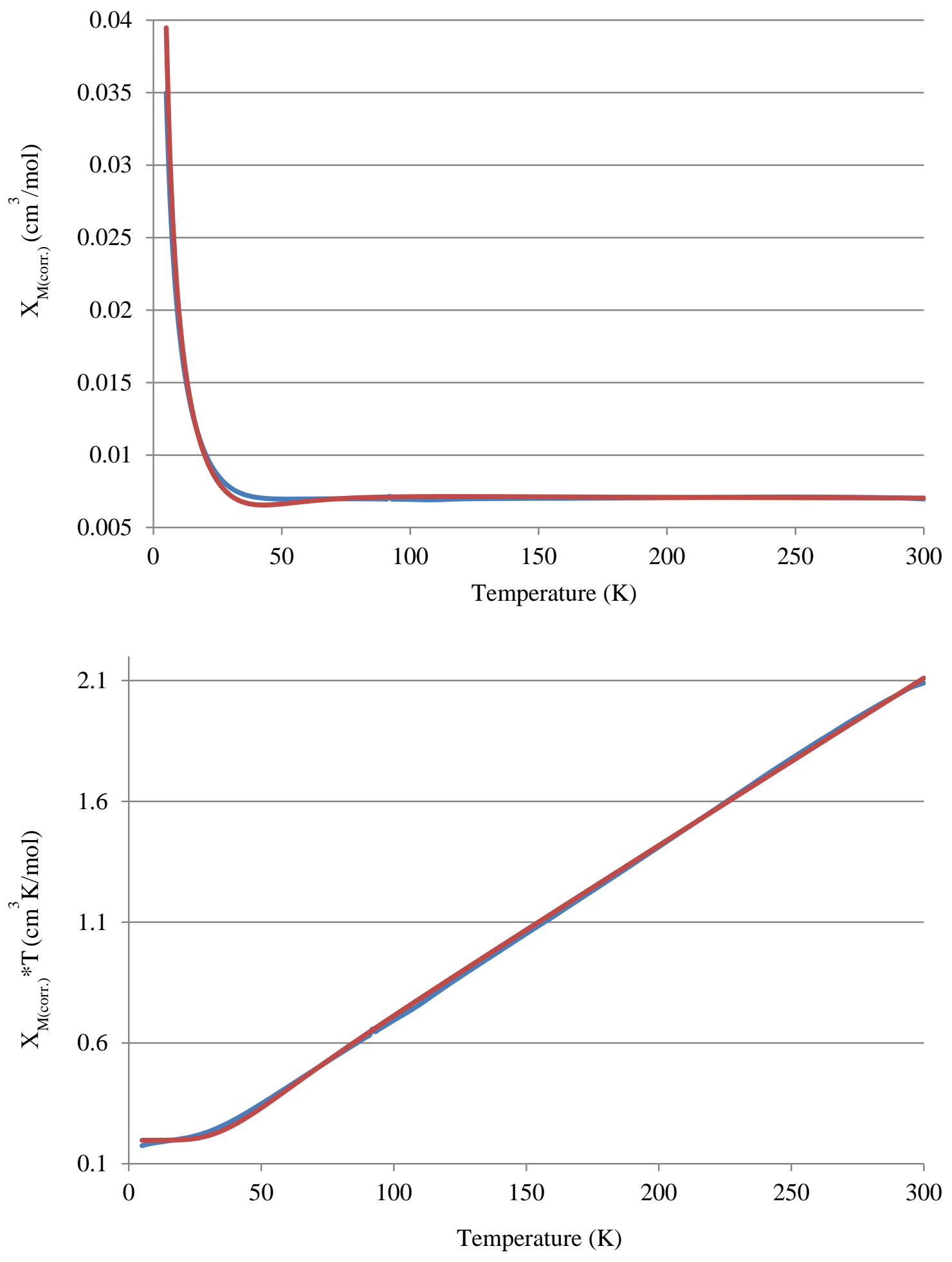

Figure S30: $\quad$ SQUID of $\left[\left\{\mathrm{Mn}\left(\mathrm{CH}_{2} \mathrm{SiMe}_{3}\right)\left(\mu-\mathrm{CH}_{2} \mathrm{SiMe}_{3}\right)\right\}_{2}(\mu-d m p e)\right](5)$ (blue) with fit to van Vleck equation $^{[5]}$ (red) for $\chi_{(M, \text { corrected) }}$ vs. T (top) and $\chi_{(M, \text { corrected) }} *$ T vs. T (bottom) 

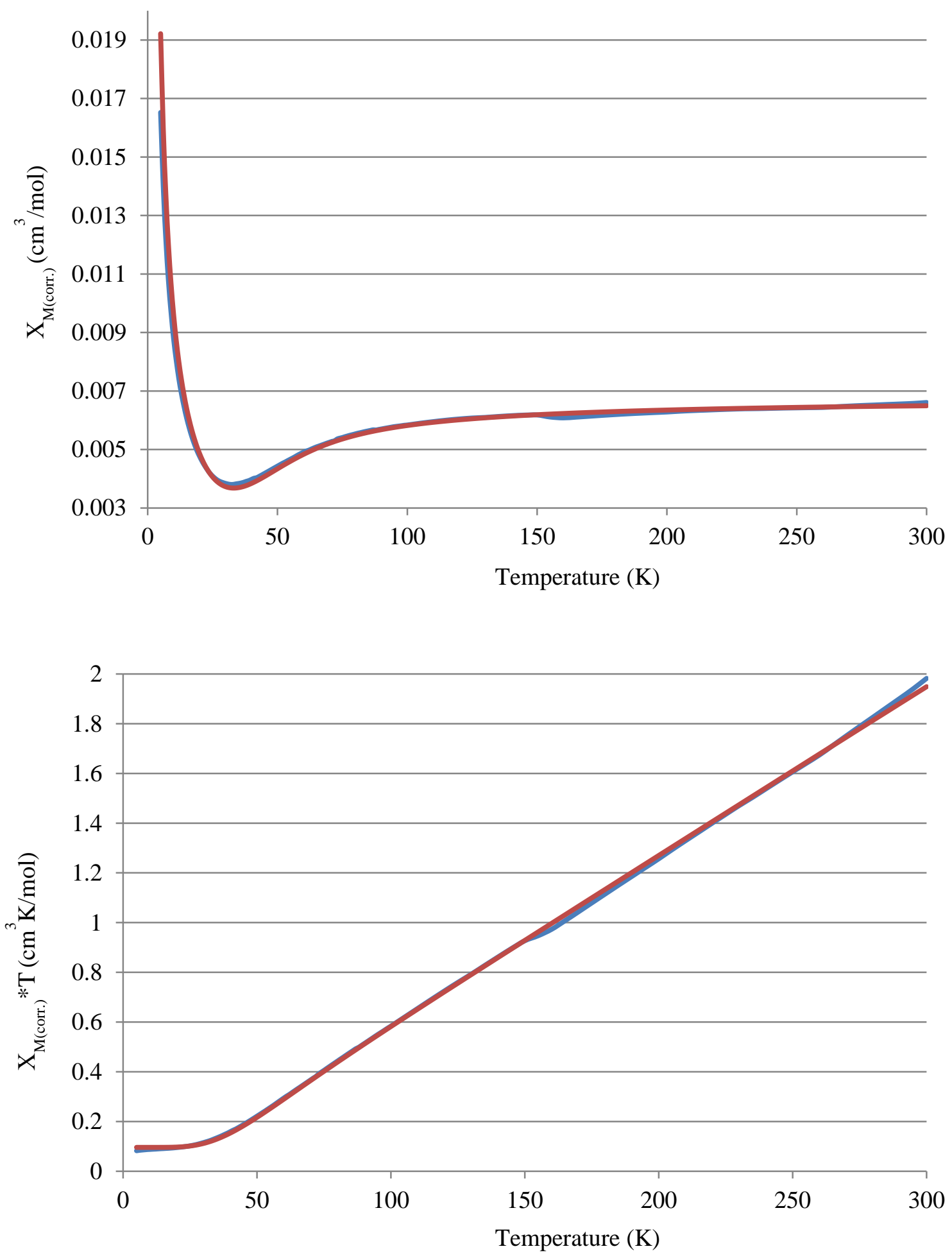

Figure S31: $\quad$ SQUID of [ $\left.\left\{\mathrm{Mn}\left(\mathrm{CH}_{2} \mathrm{CMe}_{3}\right)\left(\mu-\mathrm{CH}_{2} \mathrm{CMe}_{3}\right)\right\}_{2}(\mu-\mathrm{dmpe})\right](6)$ (blue) with fit to van Vleck equation $^{[5]}$ (red) for $\chi_{(M, \text { corrected) }}$ vs. T (top) and $\chi_{(M, \text { corrected) }} *$ T vs. T (bottom) 

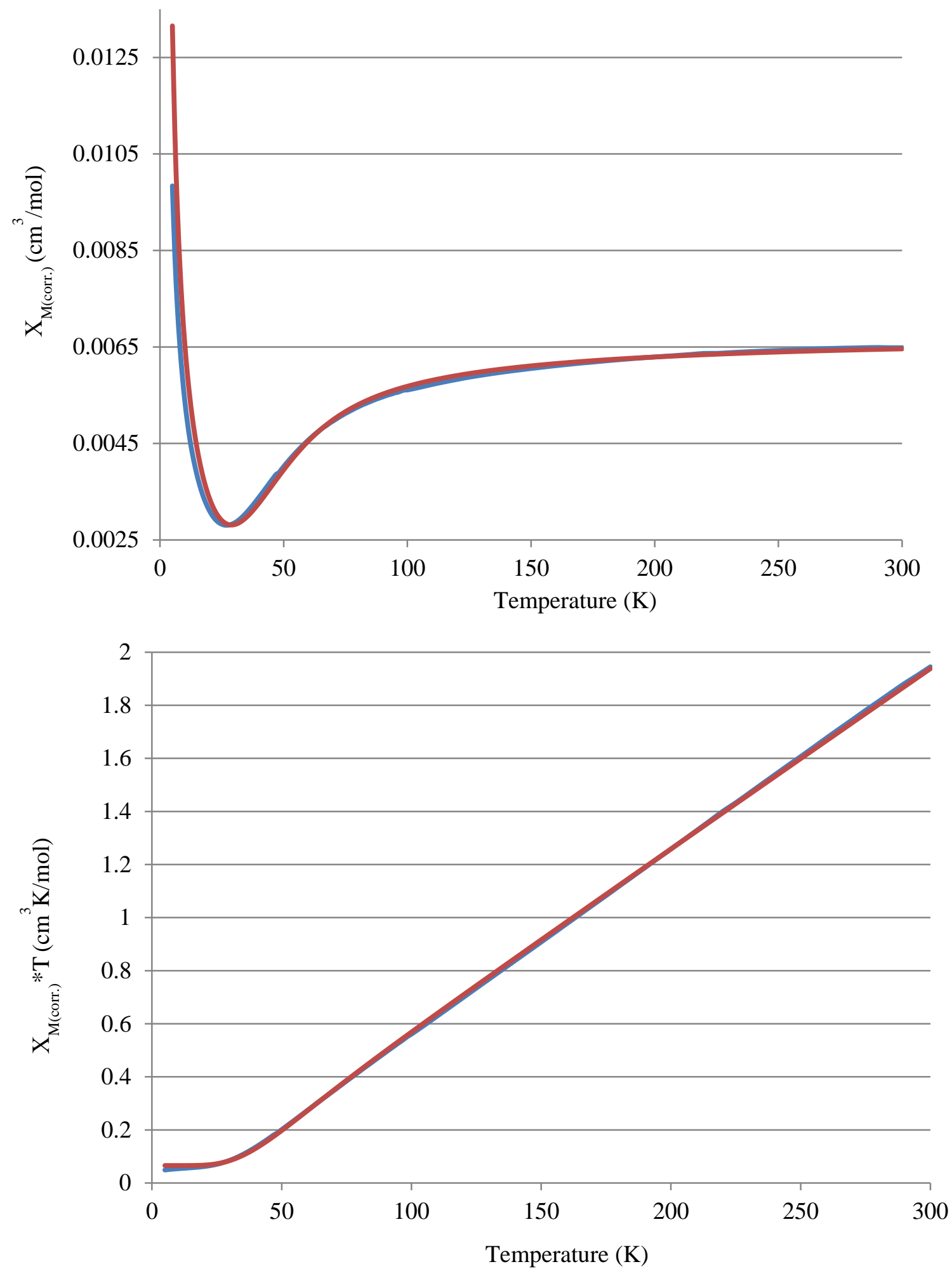

Figure S32: $\quad$ SQUID of $\left[\left\{\mathrm{Mn}\left(\mathrm{CH}_{2} \mathrm{SiMe}_{3}\right)\left(\mu-\mathrm{CH}_{2} \mathrm{SiMe}_{3}\right)\right\}_{2}(\mu-\mathrm{dmpm})\right]$ (7) (blue) with fit to van Vleck equation $^{[5]}$ (red) for $\chi_{(M, \text { corrected })}$ vs. $T$ (top) and $\chi_{(M, \text { corrected) }} *$ T vs. T (bottom) 

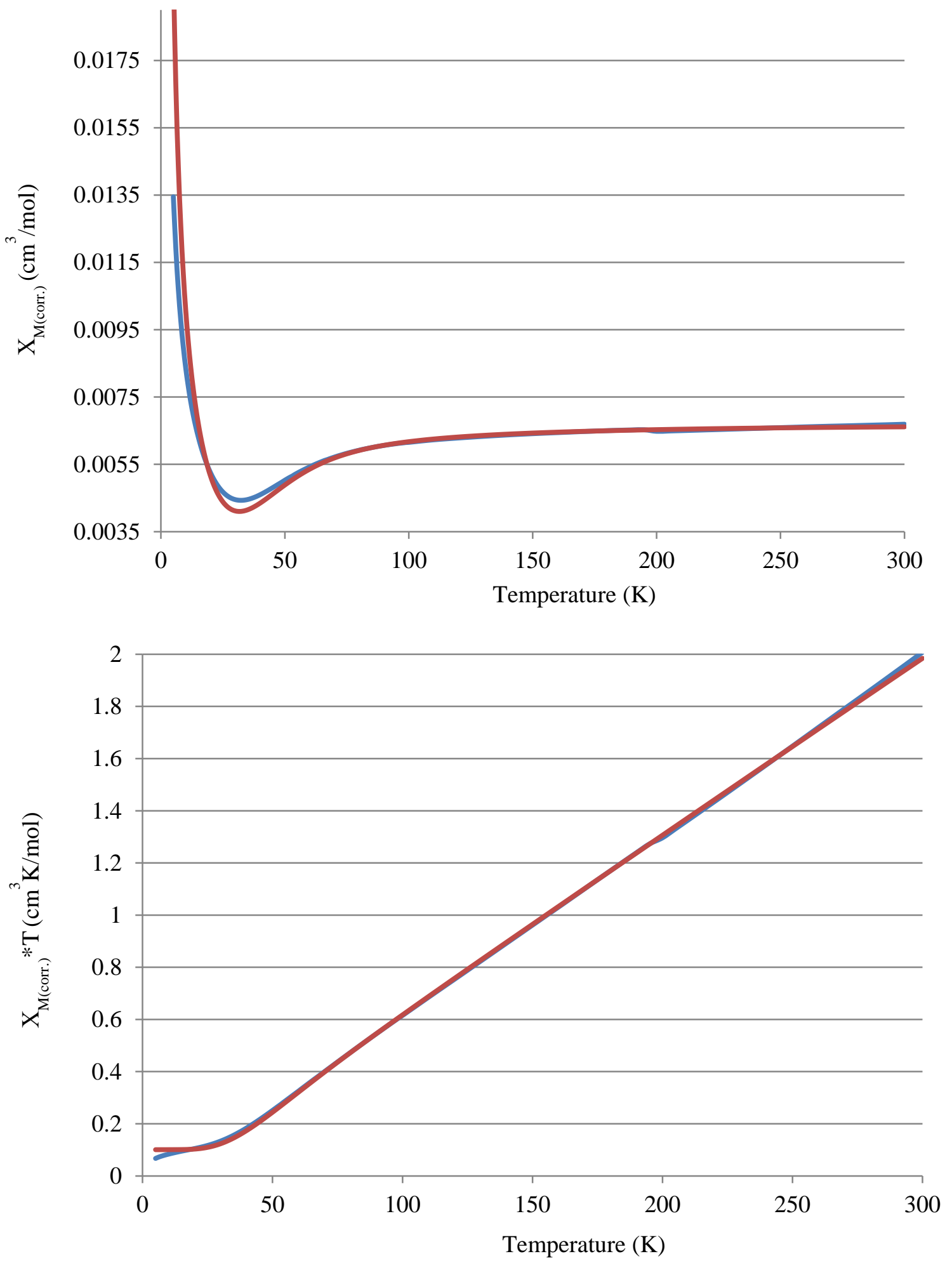

Figure S33: $\quad$ SQUID of $\left[\left\{\mathrm{Mn}\left(\mathrm{CH}_{2} \mathrm{CMe}_{3}\right)\left(\mu-\mathrm{CH}_{2} \mathrm{CMe}_{3}\right)\right\}_{2}(\mu-\mathrm{dmpm})\right](8)$ (blue) with fit to van Vleck equation $^{[5]}$ (red) for $\chi_{(M, \text { corrected })}$ vs. $T$ (top) and $\chi_{(M, \text { corrected) }} * T$ vs. T (bottom) 
Figures S34: UV/Vis spectra of compounds $2-3$ and $5-8$

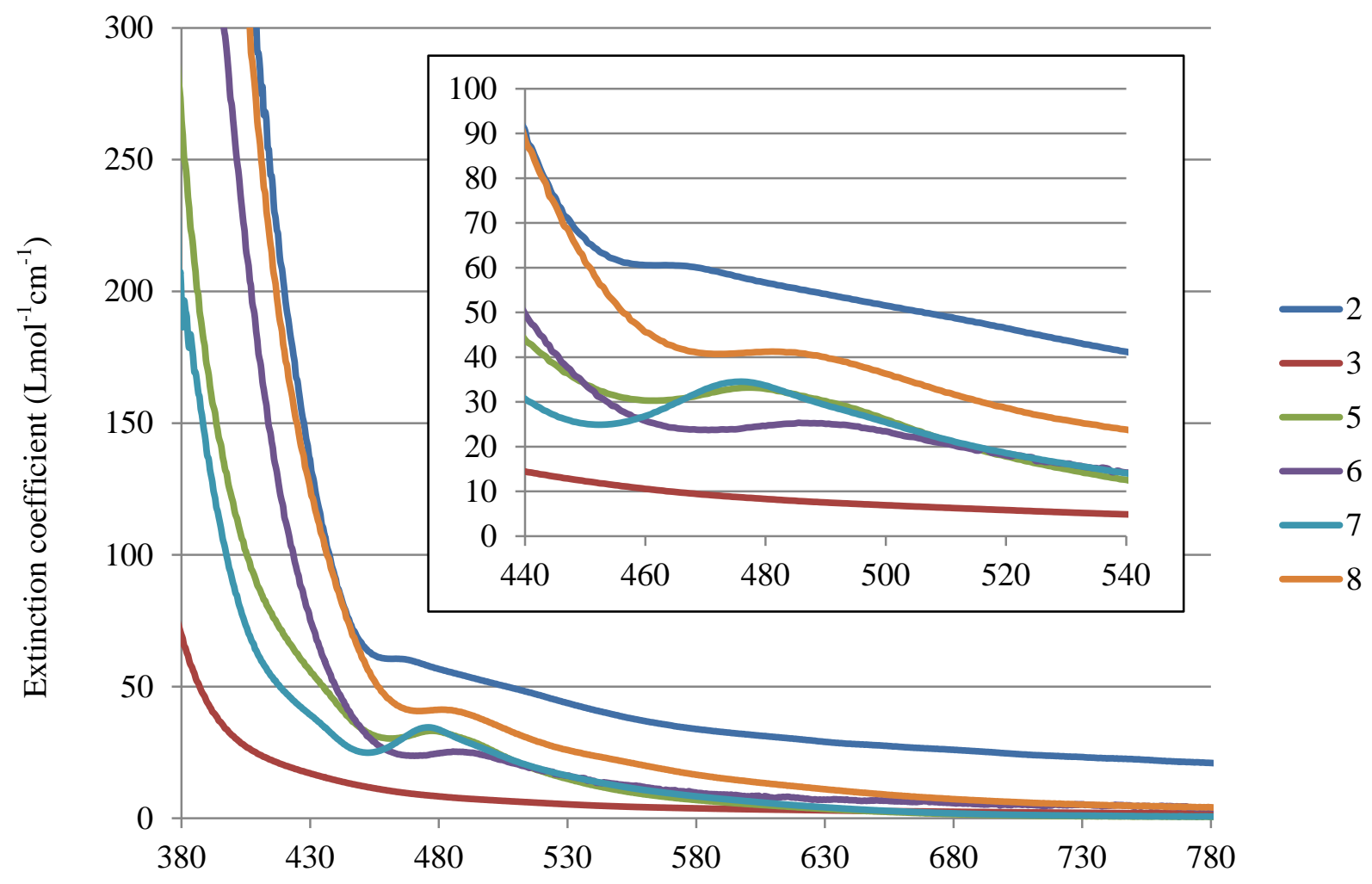

Wavelength (nm)

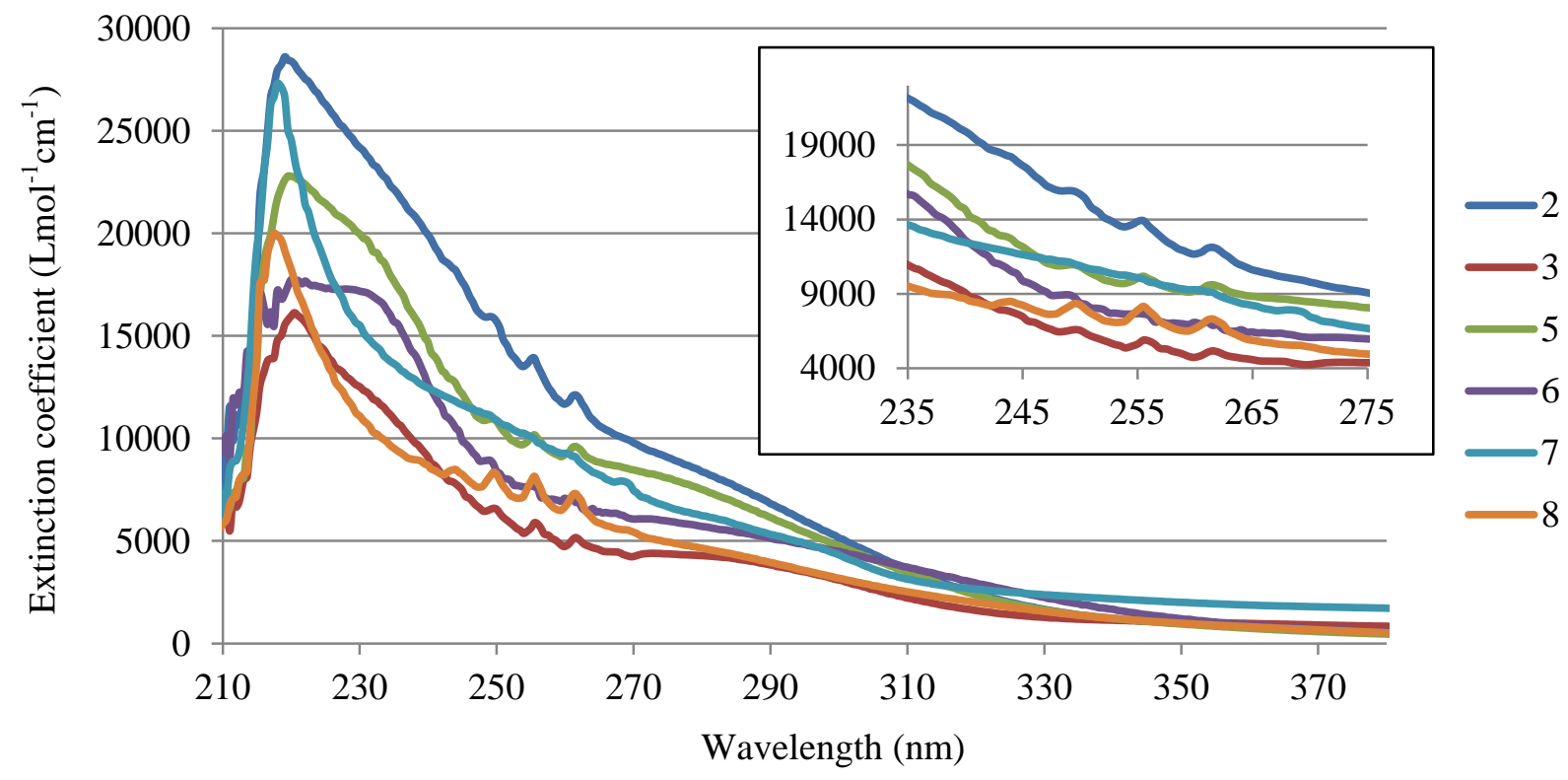

Figure S34 Electronic spectra for compounds $2-3$ and 5 - 8 (above: visible, below: UV) 
$*$ red $=$ manganese, purple $=$ silicon, green $=$ phosphorous, brown $=$ carbon

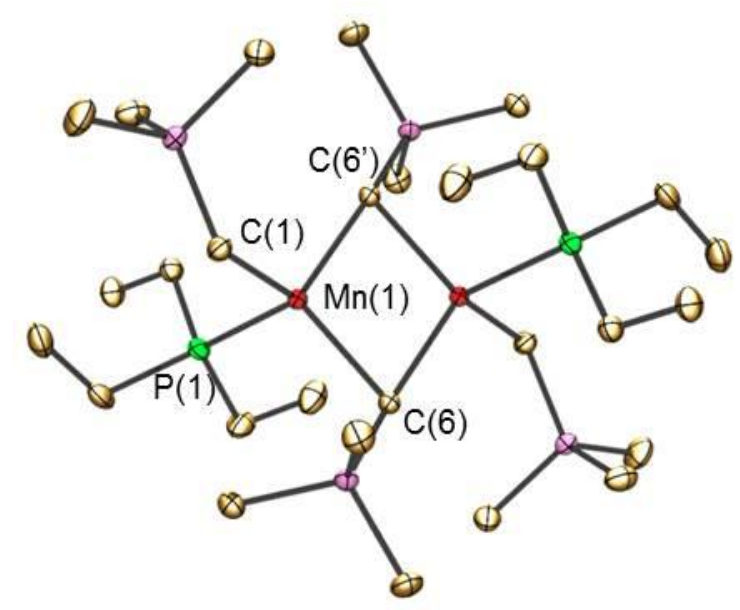

Figure S35: $\quad \mathrm{X}$-ray crystal structure for $\left[\left\{\mathrm{Mn}\left(\mathrm{CH}_{2} \mathrm{SiMe}_{3}\right)\left(\mu-\mathrm{CH}_{2} \mathrm{SiMe}_{3}\right)\left(\mathrm{PEt}_{3}\right)\right\}_{2}\right]$; bond distances (A) and angles (deg): Mn-Mn 2.7937(3), Mn-P 2.6473(4), Mn-C(1) 2.136(1), MnC(6) 2.215(1), Mn-C(6') 2.3588(9), Mn-C-Mn 75.21(3). Hydrogen atoms are omitted for clarity and thermal ellipsoids are shown at $50 \%$ probability.

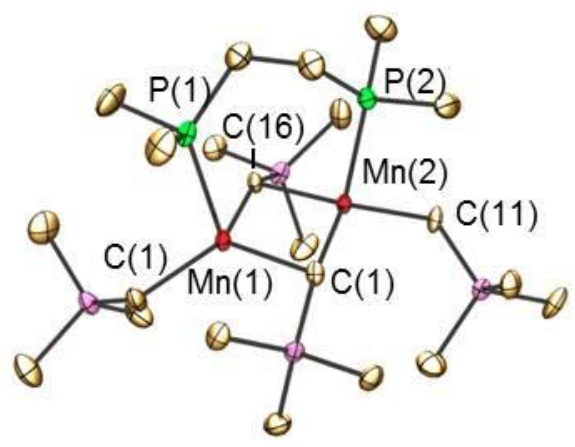

Figure S36: X-ray crystal structure for $\left[\left\{\mathrm{Mn}\left(\mathrm{CH}_{2} \mathrm{SiMe}_{3}\right)\left(\mu-\mathrm{CH}_{2} \mathrm{SiMe}_{3}\right)\right\}_{2}(\mu\right.$-dmpe $\left.)\right]$ (5) when crystallized from hexanes (crystals grown from toluene produced a different unit

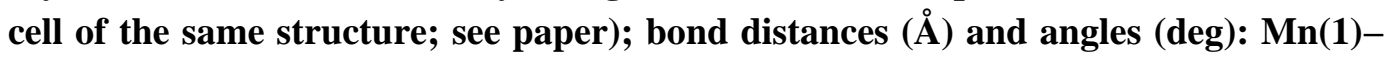
$M n(2)$ 2.7255(9), Mn(1)-P(1) 2.581(1), Mn(2)-P(2) 2.603(2), Mn(1)-C(1) 2.133(4), $M n(2)-C(11) 2.141(5), M n(1)-C(6)$ 2.211(4), $M n(2)-C(16) 2.210(4), M n(1)-C(16)$ 2.379(4), Mn(2)-C(6) 2.372(4), Mn(1)-C(6)-Mn(2) 72.9(1), Mn(1)-C(16)-Mn(2) 72.8(1). Hydrogen atoms are omitted for clarity and thermal ellipsoids are shown at $50 \%$ probability. 

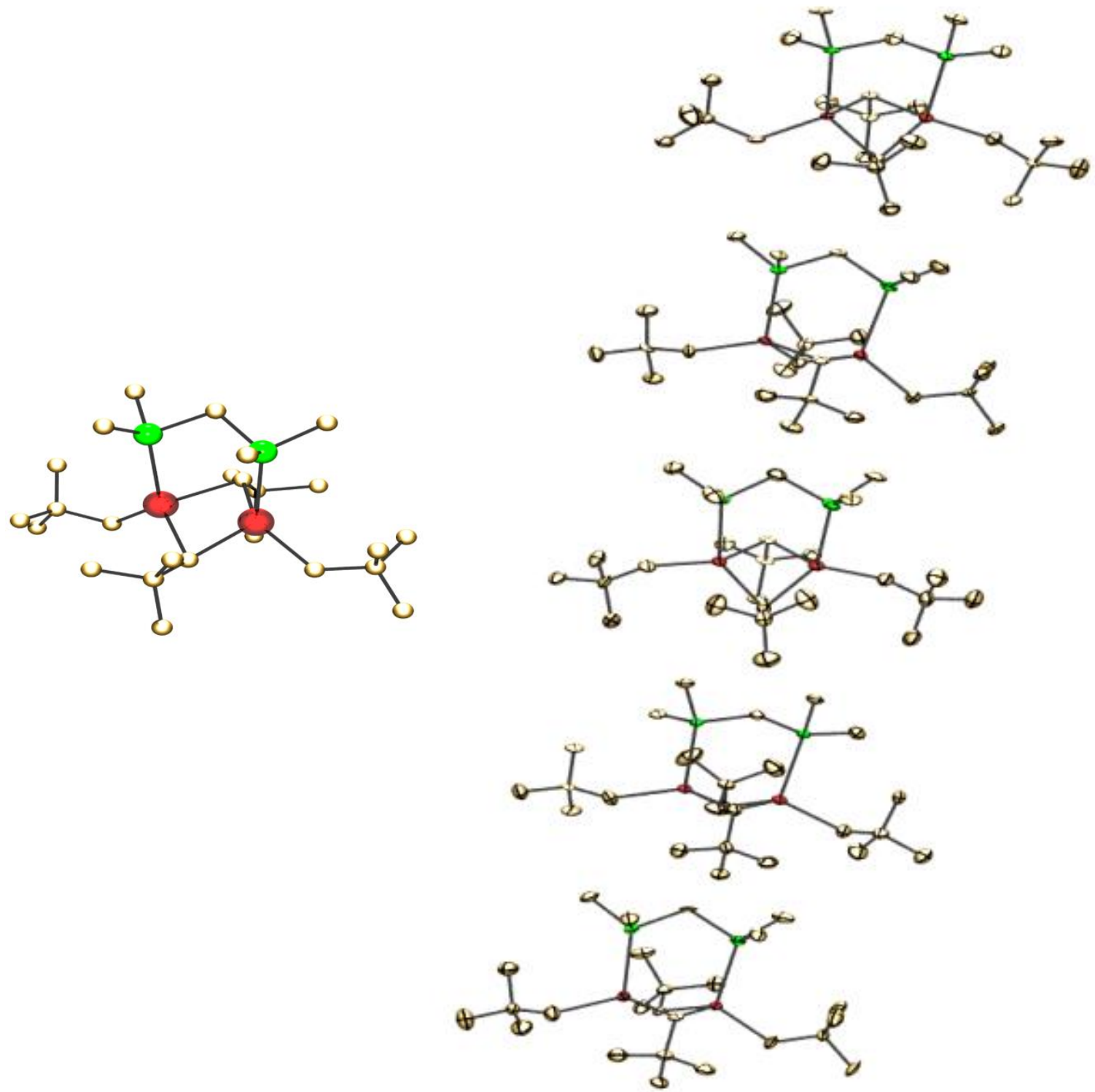

Figure S37: Left: Single crystal X-ray structure of $\left[\left\{\mathrm{Mn}\left(\mathrm{CH}_{2} \mathrm{CMe}_{3}\right)\left(\mu-\mathrm{CH}_{2} \mathrm{CMe}_{3}\right)\right\}_{2}(\mathrm{dmpm})\right](8)$ crystallized by cooling a saturated solutions of hexanes at $-30^{\circ} \mathrm{C}$. Crystal structure used for determining connectivity but not useful for determining bond metrics. All ligands are disordered and only the dominant disorder is shown. Hydrogen atoms are omitted for clarity.

Right: Single crystal X-ray structure of $\left[\left\{\mathrm{Mn}\left(\mathrm{CH}_{2} \mathrm{CMe}_{3}\right)\left(\mu-\mathrm{CH}_{2} \mathrm{CMe}_{3}\right)\right\}_{2}(\mathrm{dmpm})\right](8)$ crystallized by slow evaporation of hexanes from a diluted solution (5 unique molecules in the asymmetric unit). Crystal structure used for determining connectivity but not useful for determining bond metrics. Hydrogen atoms are omitted for clarity. 
Figures S38-S45: $\quad$ CIF reports for compounds $2-7$ and $\left[\left\{\mathrm{Mn}\left(\mathrm{CH}_{2} \underline{\mathrm{SiMe}}_{3}\right)\left(\mu-\mathrm{CH}_{2} \underline{\mathrm{SiMe}}_{3}\right)\right.\right.$ $\left.\underline{\text { PEt }}_{3} \underline{H}_{2}\right]$

\section{checkCIF/PLATON report}

Structure factors have been supplied for datablock(s) npf

THIS REPORT IS FOR GUIDANCE ONLY. IF USED AS PART OF A REVIEW PROCEDURE FOR PUBLICATION, IT SHOULD NOT REPLACE THE EXPERTISE OF AN EXPERIENCED CRYSTALLOGRAPHIC REFEREE.

No syntax enrors found. CIF dictionary Interpreting this report

\section{Datablock: npf}

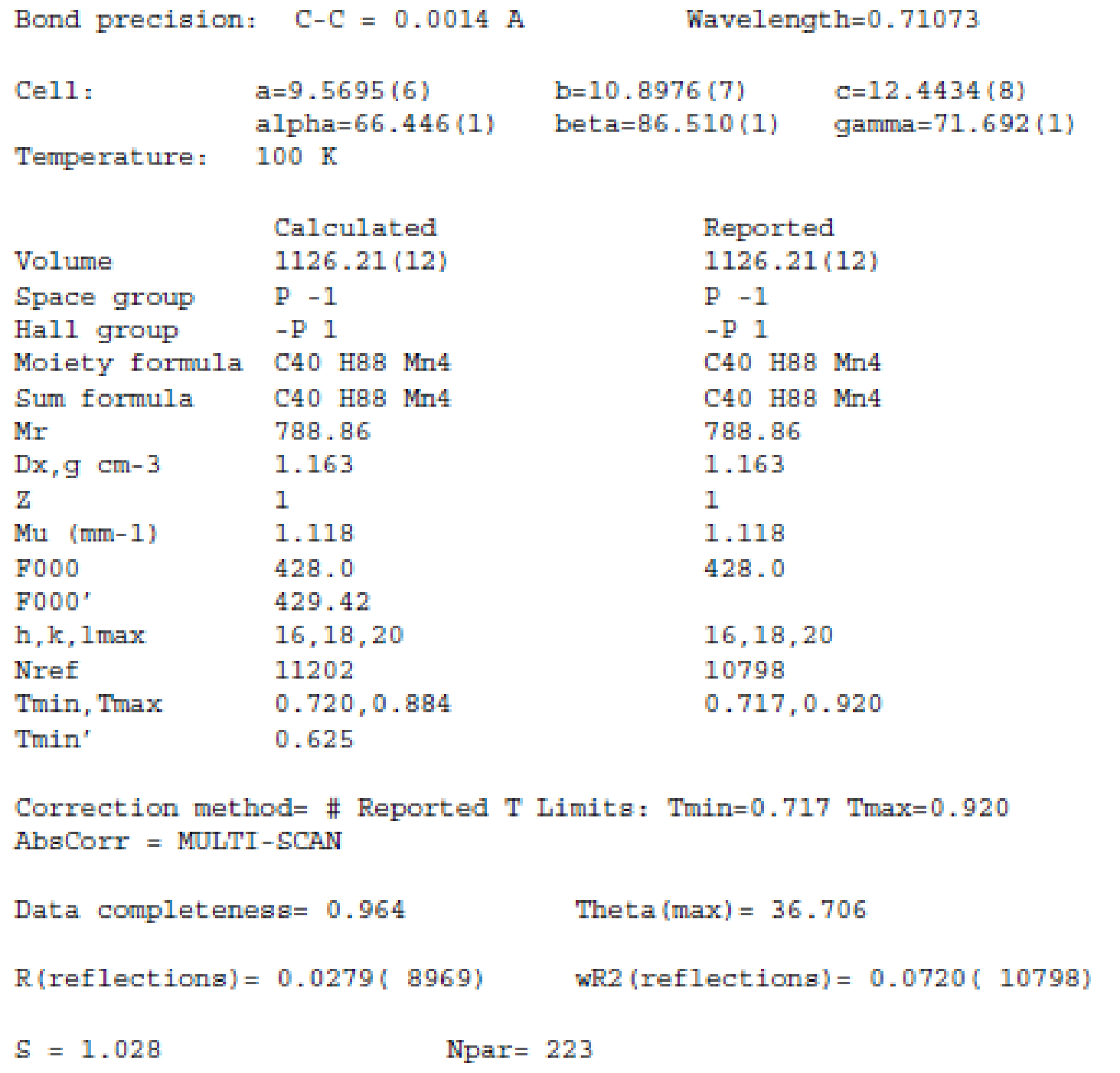

Figure S38: CIF report for $\left[\left\{\mathrm{Mn}\left(\mathrm{CH}_{2} \mathrm{CMe}_{3}\right)\left(\mu-\mathrm{CH}_{2} \mathrm{CMe}_{3}\right)_{2}\right\}_{2}\left\{\mathrm{Mn}\left(\mu-\mathrm{CH}_{2} \mathrm{CMe}_{3}\right)_{2} \mathrm{Mn}\right\}\right](2)$ 


\section{checkCIF/PLATON report}

Structure factors have been supplied for datablock(s) nse

THIS REPORT IS FOR GUIDANCE ONLY. IF USED AS PART OF A REVIEW PROCEDURE

FOR PUBLICATION, IT SHOULD NOT REPLACE THE EXPERTISE OF AN EXPERIENCED

CRYSTALLOGRAPHIC REFEREE.

No syntax enrors found. CIF dictionary Interpreting this report

\section{Datablock: nse}

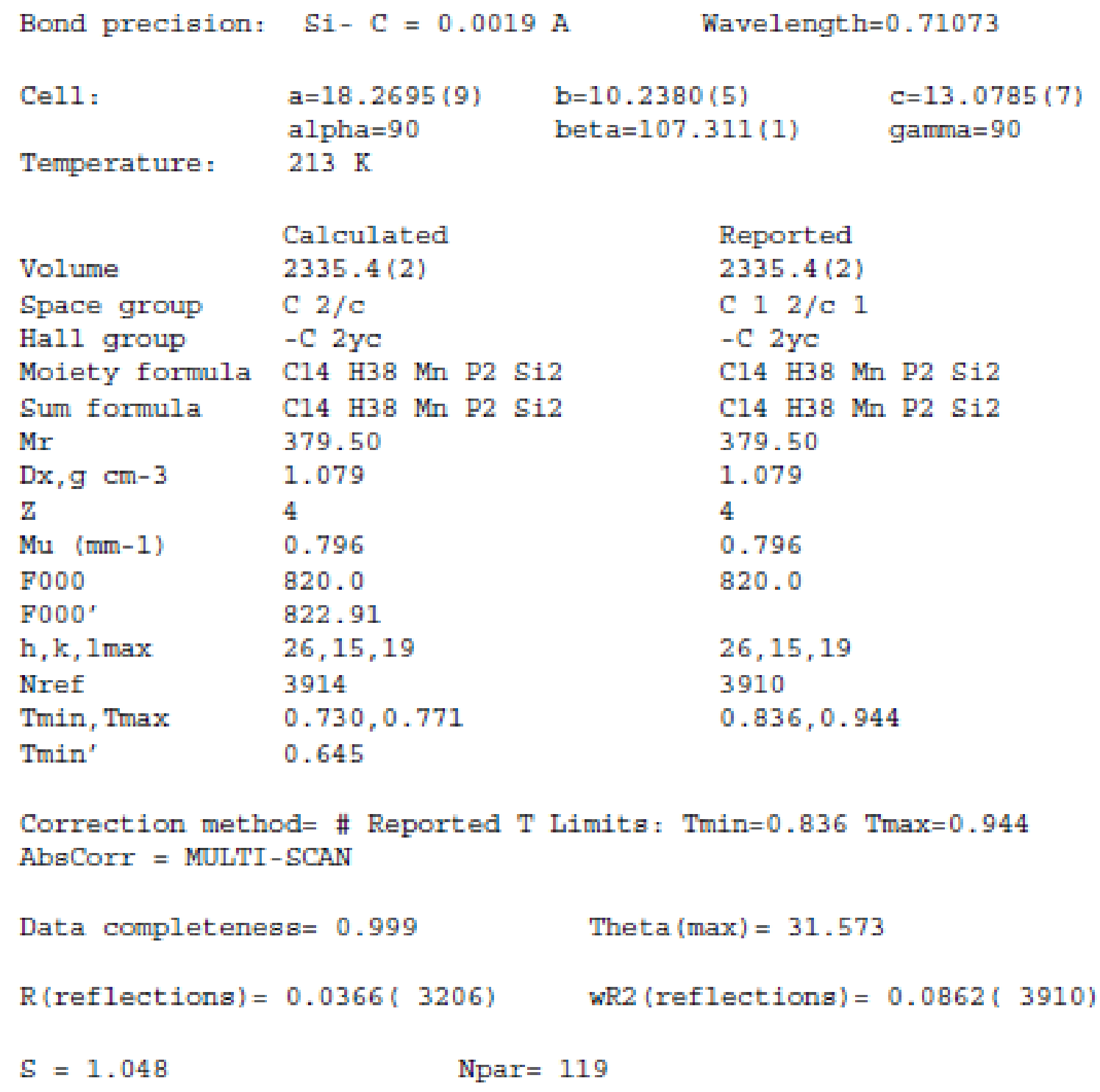

Figure S39: CIF report for $\left[\mathrm{Mn}\left(\mathrm{CH}_{2} \mathrm{SiMe}_{3}\right)_{2}(\mathrm{dmpe})\right](3)$ 


\section{checkCIF/PLATON report}

Structure factors have been supplied for datablock(s) npe

THIS REPORT IS FOR GUIDANCE ONLY. IF USED AS PART OF A REVIEW PROCEDURE FOR PUBLICATION, IT SHOULD NOT REPLACE THE EXPERTISE OF AN EXPERIENCED CRYSTALIOGRAPHIC REFEREE.

No syntax enrors found. CIF dictionary Interpreting this report

\section{Datablock: npe}

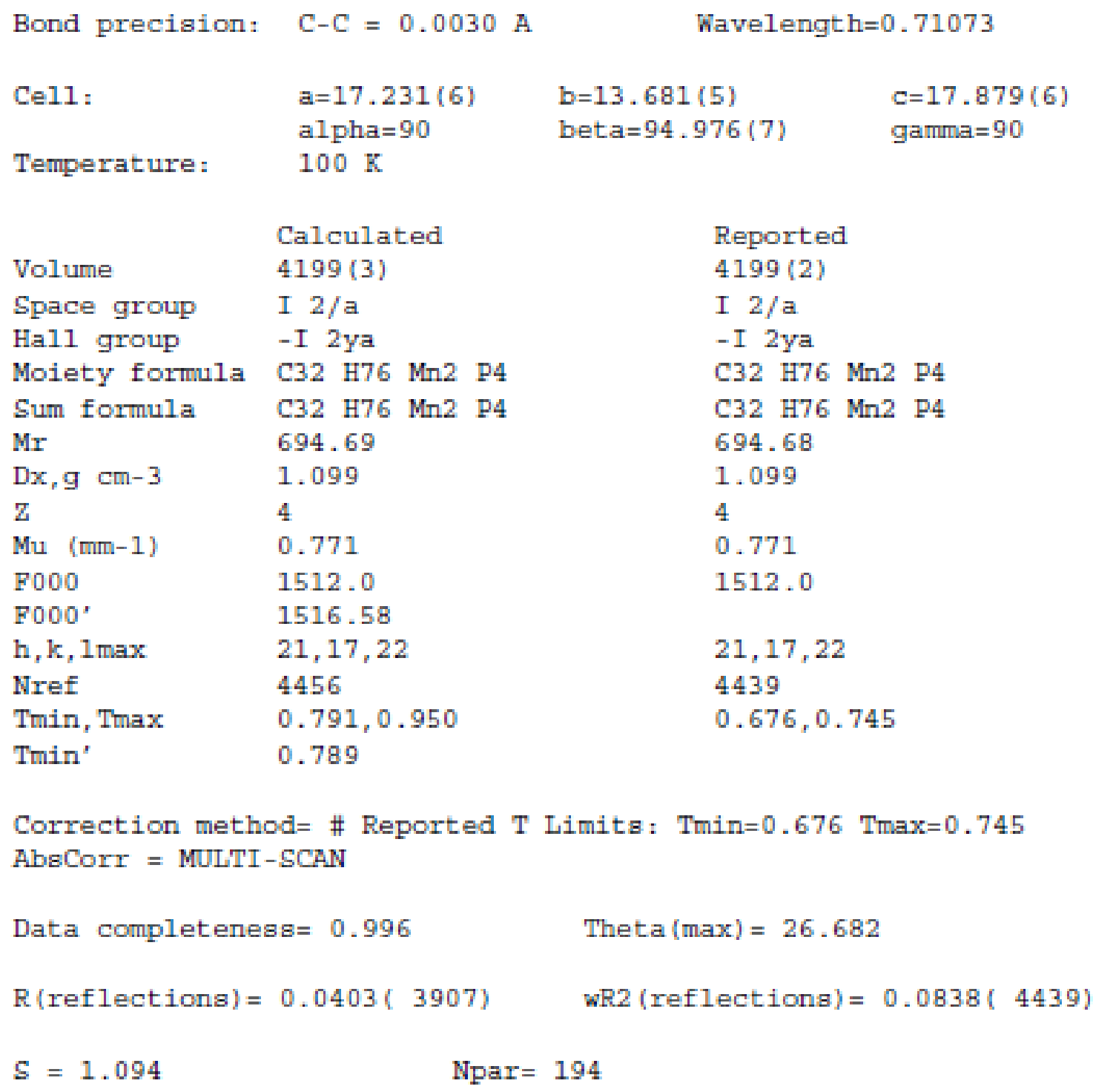

Figure S40: CIF report for $\left[\left\{\mathrm{Mn}\left(\mathrm{CH}_{2} \mathrm{CMe}_{3}\right)_{2}(\mu \text {-dmpe })\right\}_{2}\right](4)$ 


\section{checkCIF/PLATON report}

Structure factors have been supplied for datablock(s) nsb_hex

THIS REPORT IS FOR GUIDANCE ONLY. IF USED AS PART OF A REVIEW PROCEDURE FOR PUBLICATION, IT SHOULD NOT REPLACE THE EXPERTISE OF AN EXPERIENCED CRYSTALLOGRAPHIC REFEREE.

No syntax enrors found. CIF dictionary Interpreting this report

Datablock: nsb_hex

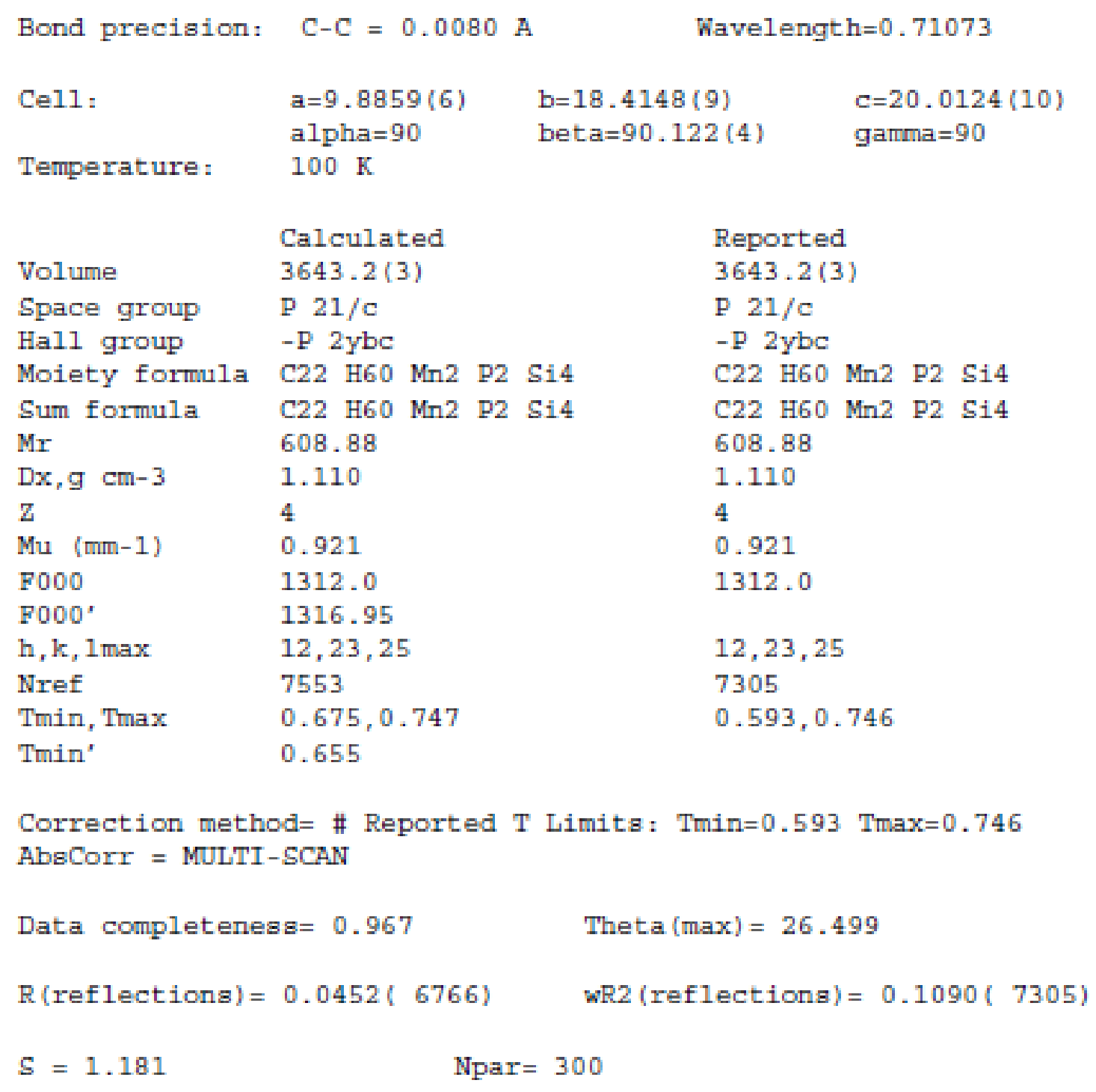

Figure S41: CIF report for $\left[\left\{\mathrm{Mn}\left(\mathrm{CH}_{2} \mathrm{SiMe}_{3}\right)\left(\mu-\mathrm{CH}_{2} \mathrm{SiMe}_{3}\right)\right\}_{2}(\mu\right.$-dmpe $\left.)\right]$ (5) crystallized from hexanes 


\section{checkCIF/PLATON report}

Structure factors have been supplied for datablock(s) nsb_tol

THIS REPORT IS FOR GUIDANCE ONLY. IF USED AS PART OF A REVIEW PROCEDURE FOR PUBLICATION, IT SHOULD NOT REPLACE THE EXPERTISE OF AN EXPERIENCED CRYSTALLOGRAPHIC REFEREE.

No syntax enrors found. CIF dictionary Interpreting this report

\section{Datablock: nsb_tol}

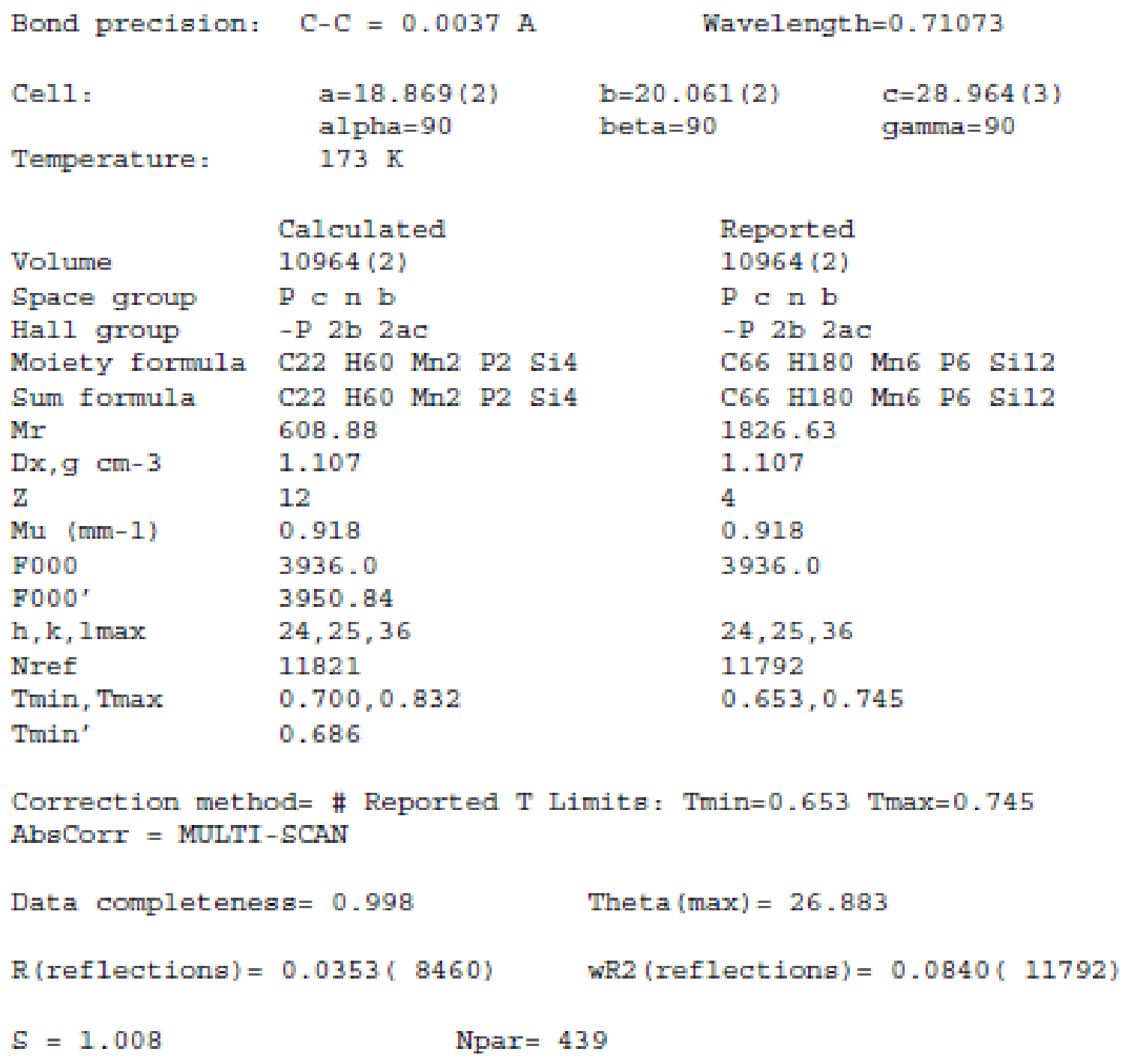

Figure S42: CIF report for $\left[\left\{\mathrm{Mn}\left(\mathrm{CH}_{2} \mathrm{SiMe}_{3}\right)\left(\mu-\mathrm{CH}_{2} \mathrm{SiMe}_{3}\right)\right\}_{2}(\mu\right.$-dmpe $\left.)\right]$ (5) crystallized from toluene 


\section{checkCIF/PLATON report}

Structure factors have been supplied for datablock(s) npb

THIS REPORT IS FOR GUIDANCE ONLY. IF USED AS PART OF A REVIEW PROCEDURE

FOR PUBLICATION, IT SHOULD NOT REPLACE THE EXPERTISE OF AN EXPERIENCED

CRYSTALLOGRAPHIC REFEREE.

No syntax enrors found. CIF dictionary Interpreting this report

\section{Datablock: npb}

\begin{tabular}{|c|c|c|}
\hline Bond precision: & $C-C=0.0084 \mathrm{~A}$ & Wavelength $=0.71073$ \\
\hline Cell: & $\begin{array}{l}a=11 \cdot 94900 \\
\text { alpha }=90\end{array}$ & $\begin{array}{ll}\mathrm{b}=14.91500 & \mathrm{c}=18.31200 \\
\text { bet } \mathrm{a}=92.9800 & \text { gamma }=90\end{array}$ \\
\hline \multirow[t]{2}{*}{ Temperature: } & $173 \mathrm{~K}$ & \\
\hline & Calculated & Reported \\
\hline Volume & 3259.139 & 3259 \\
\hline Space group & p $21 / \mathrm{c}$ & p $21 / c$ \\
\hline Hall group & -p 2 ybc & $-\mathrm{p} 2 \mathrm{ybc}$ \\
\hline Moiety formula & $\mathrm{C} 26 \mathrm{H} 60 \mathrm{Mn} 2 \mathrm{P} 2$ & $\mathrm{C} 26 \mathrm{H} 60 \mathrm{Mn} 2 \mathrm{P} 2$ \\
\hline Sum formula & $\mathrm{C} 26 \mathrm{H} 60 \mathrm{Mn} 2 \mathrm{P} 2$ & $\mathrm{C} 26 \mathrm{H} 60 \mathrm{Mn} 2 \mathrm{P} 2$ \\
\hline $\mathrm{Mr}$ & 544.56 & 544.56 \\
\hline $\mathrm{Dx}, \mathrm{g} \mathrm{cm}-3$ & 1.110 & 1.110 \\
\hline $\mathrm{z}$ & 4 & 4 \\
\hline Mu (mm-1) & 0.883 & 0.883 \\
\hline F000 & 1184.0 & 1184.0 \\
\hline F000' & 1187.71 & \\
\hline $\mathrm{h}, \mathrm{k}, 1 \max$ & $14,17,21$ & $14,17,21$ \\
\hline Nref & 5735 & 6014 \\
\hline Tmin, Tmax & $0.670,0.776$ & $0.662,0.746$ \\
\hline 'Tmin' & 0.620 & \\
\hline \multicolumn{2}{|c|}{$\begin{array}{l}\text { Correction method= \# Reported T } \\
\text { AbsCorr = MULTI-SCAN }\end{array}$} & Himits: $T \min =0.662 \mathrm{Tmax}=0.746$ \\
\hline \multicolumn{2}{|c|}{ Data completeness $=1.049$} & Theta $(\max )=24.996$ \\
\hline $\mathrm{R}($ reflections) $=$ & $0.0518(5129)$ & $w R 2$ (reflections) $=0.1368(6014)$ \\
\hline$S=1.118$ & Npar $=$ & 320 \\
\hline
\end{tabular}

Figure S43: $\quad$ CIF report for $\left[\left\{\mathrm{Mn}\left(\mathrm{CH}_{2} \mathrm{CMe}_{3}\right)\left(\mu-\mathrm{CH}_{2} \mathrm{CMe}_{3}\right)\right\}_{2}(\mu\right.$-dmpe $\left.)\right](6)$ 


\section{checkCIF/PLATON report}

Structure factors have been supplied for datablock(s) nsm

THIS REPORT IS FOR GUIDANCE ONLY. IF USED AS PART OF A REVIEW PROCEDURE FOR PUBLICATION, IT SHOULD NOT REPLACE THE EXPERTISE OF AN EXPERIENCED CRYSTALLOGRAPHIC REFEREE.

No syntax enrors found. CIF dictionary Interpreting this report

\section{Datablock: nsm}

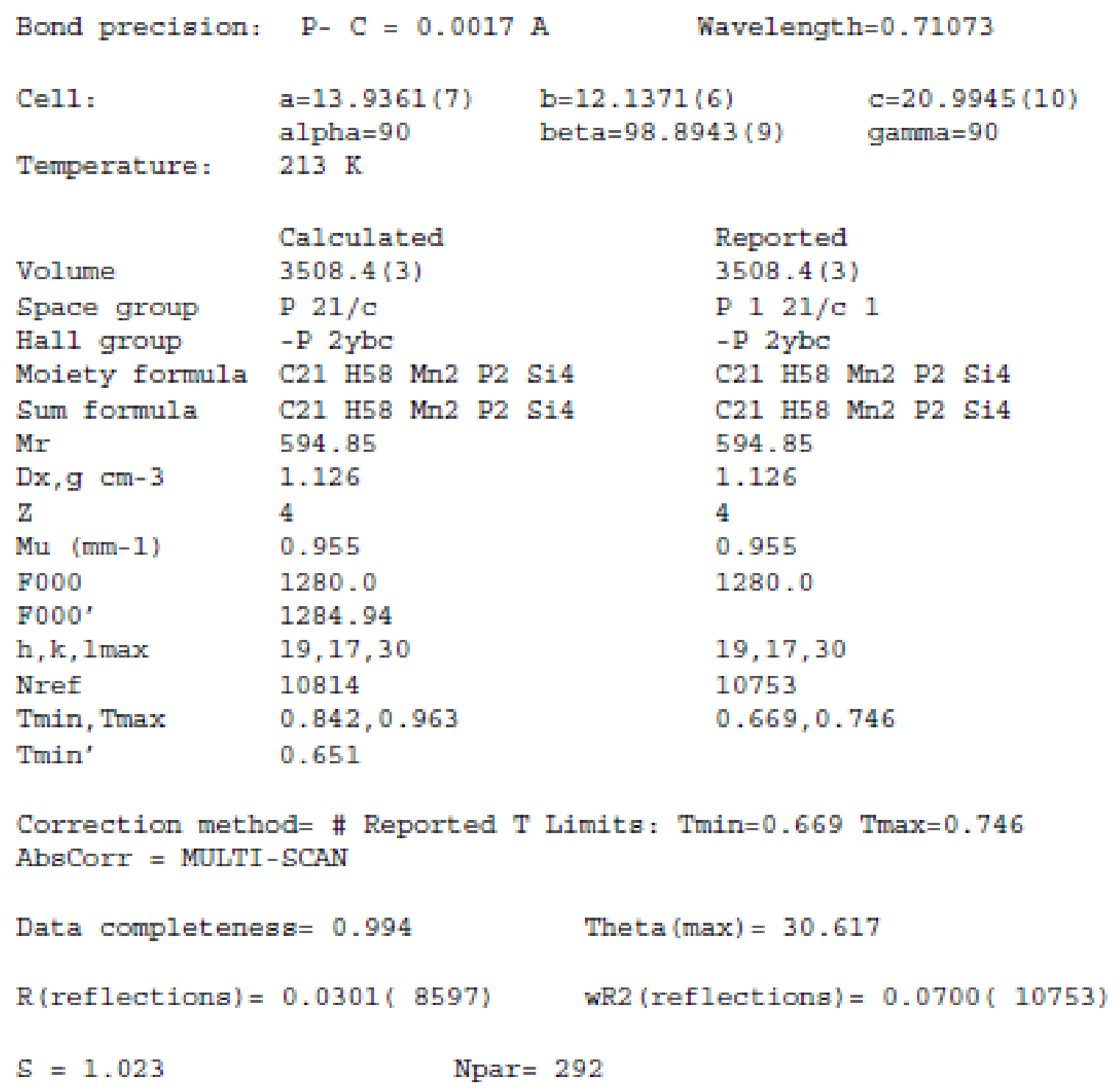

Figure S44: $\quad$ CIF report for $\left[\left\{\mathrm{Mn}\left(\mathrm{CH}_{2} \mathrm{SiMe}_{3}\right)\left(\mu-\mathrm{CH}_{2} \mathrm{SiMe}_{3}\right)\right\}_{2}(\mu-\mathrm{dmpm})\right](7)$ 


\section{checkCIF/PLATON report}

Structure factors have been supplied for datablock(s) $n s \mathrm{PEt} 3$

THIS REPORT IS FOR GUIDANCE ONLY. IF USED AS PART OF A REVIEW PROCEDURE

FOR PUBLICATION, IT SHOULD NOT REPLACE THE EXPERTISE OF AN EXPERIENCED CRYSTALLOGRAPHIC REFEREE.

No syntax enrors found. CIF dictionary Interpreting this report

\section{Datablock: nsPEt3}

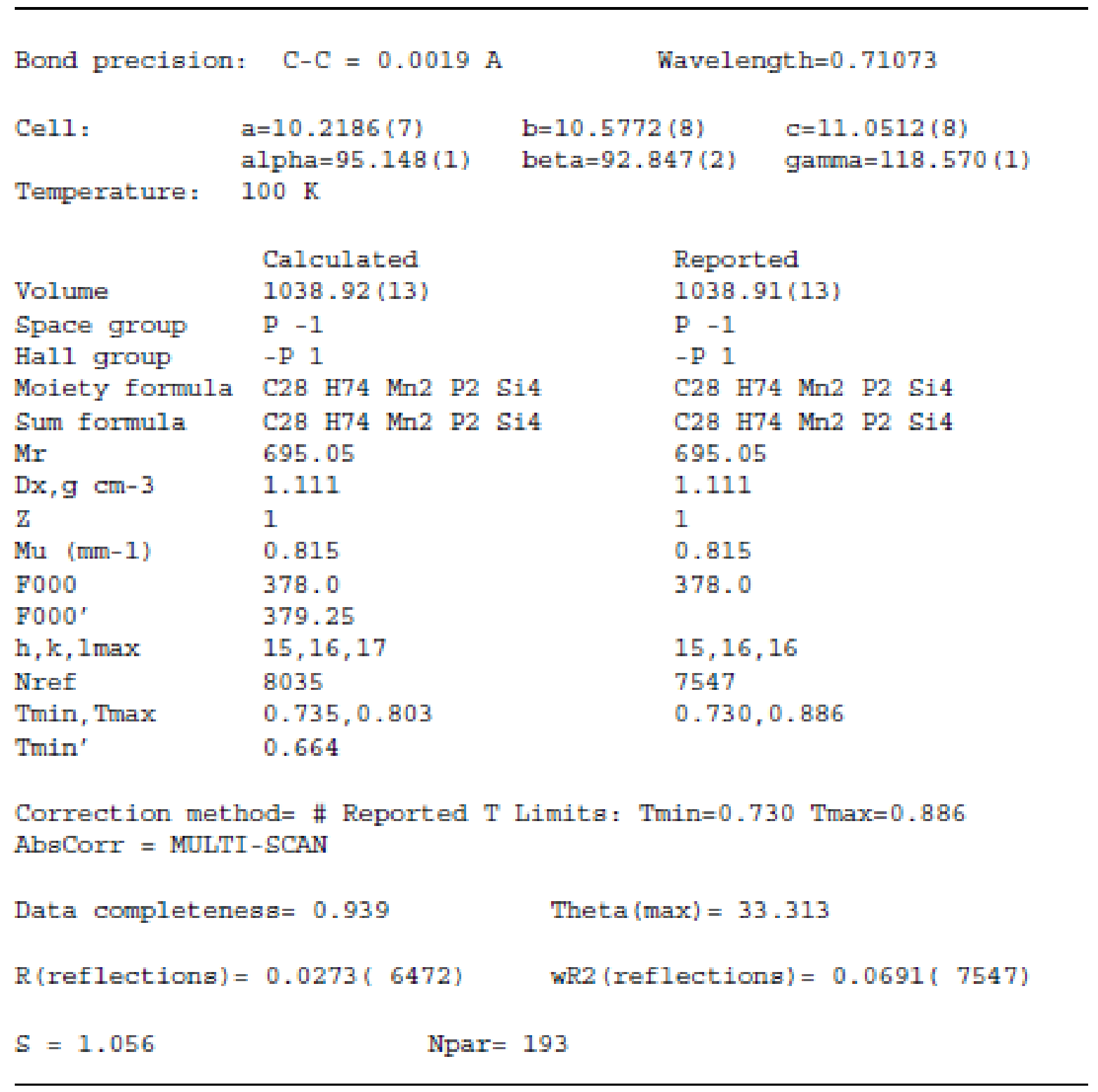

Figure S45: CIF report for $\left[\left\{\mathrm{Mn}\left(\mathrm{CH}_{2} \mathrm{SiMe}_{3}\right)\left(\mu-\mathrm{CH}_{2} \mathrm{SiMe}_{3}\right)\left(\mathrm{PEt}_{3}\right)\right\}_{2}\right]$ 
Figures S46-S55: $\quad{ }^{1} \mathrm{H}$ NMR spectra of results of $\mathrm{H}_{2} \underline{\text { reactions with } 1 \text {-8 }}$

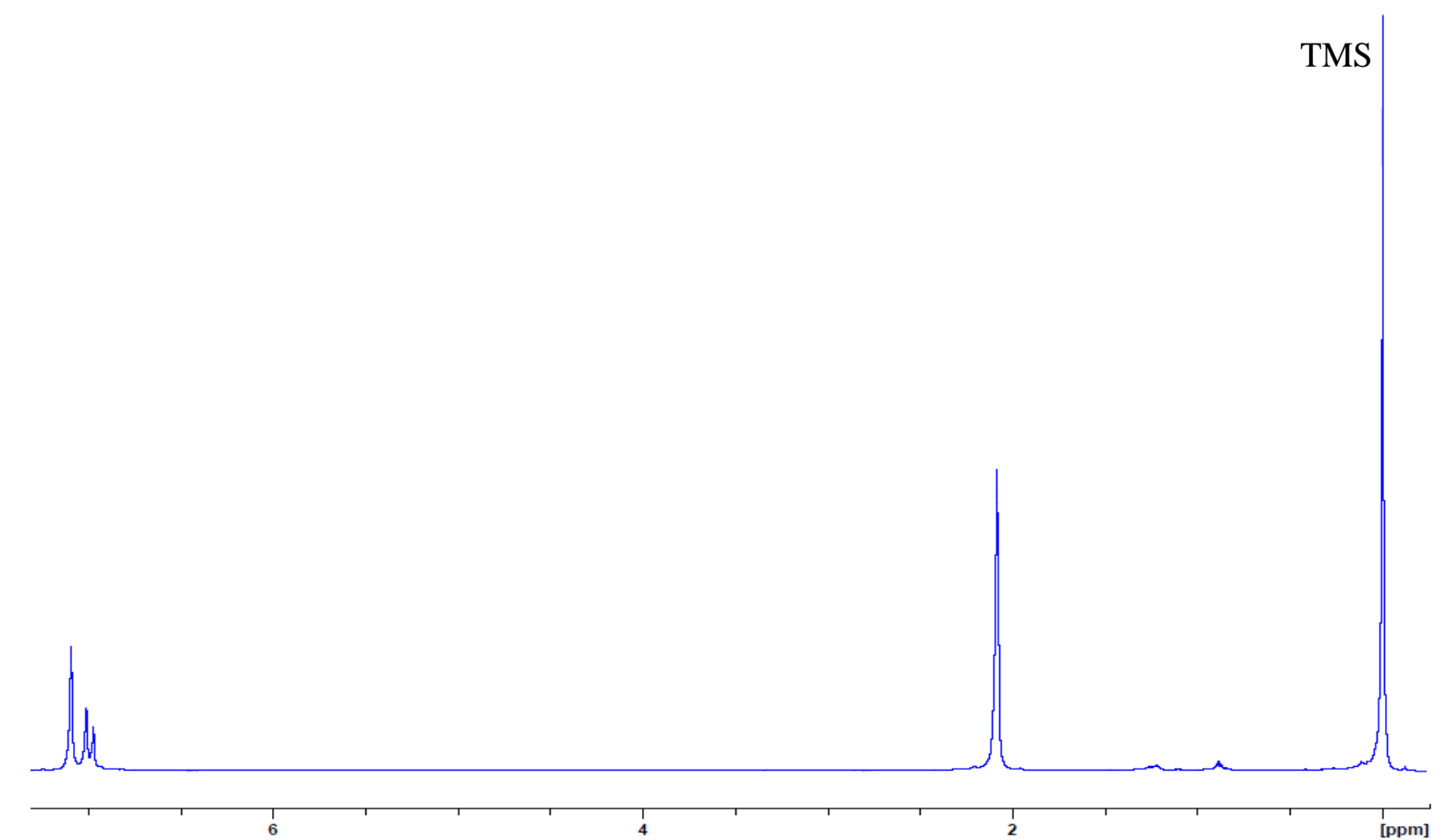

Figure S46: $\quad{ }^{1} \mathrm{H}$ NMR spectrum of $\left[\left\{\mathrm{Mn}\left(\mu-\mathrm{CH}_{2} \mathrm{SiMe}_{3}\right)_{2}\right\}_{\infty}\right](1)$ and $\mathrm{H}_{2}$ after 2 days at $120^{\circ} \mathrm{C}$ in $\mathrm{d}_{8^{-}}$ toluene (500 MHz)

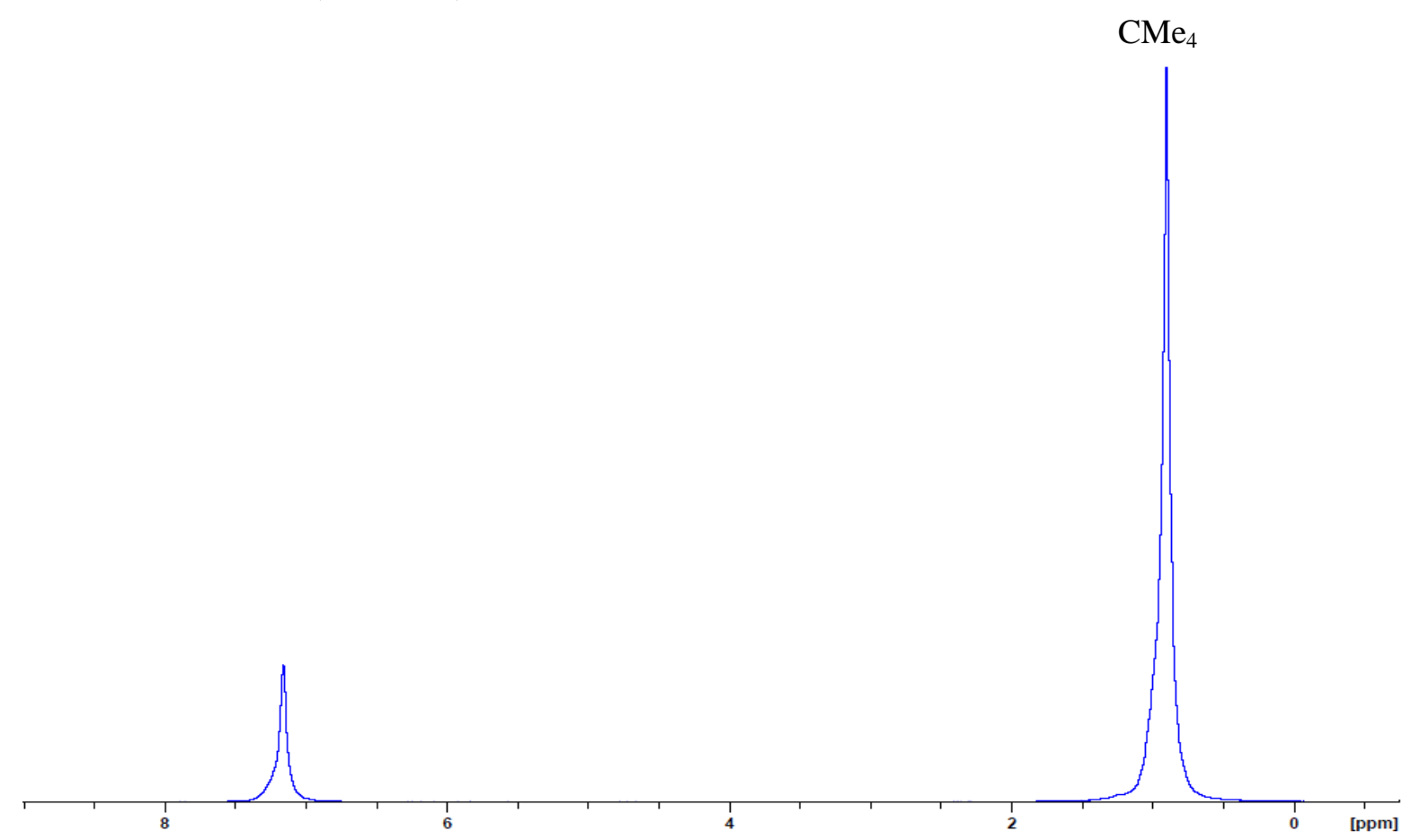

Figure S47: $\quad{ }^{1} \mathrm{H}$ NMR spectrum of $\left[\left\{\mathrm{Mn}\left(\mathrm{CH}_{2} \mathrm{CMe}_{3}\right)\left(\mu-\mathrm{CH}_{2} \mathrm{CMe}_{3}\right)_{2}\right\}_{2}\left\{\mathrm{Mn}\left(\mu-\mathrm{CH}_{2} \mathrm{CMe}_{3}\right)_{2} \mathrm{Mn}\right\}\right]$ (2) and $\mathrm{H}_{2}$ after 3 days at $25^{\circ} \mathrm{C}$ in $\mathrm{C}_{6} \mathrm{D}_{6}(500 \mathrm{MHz})$ 
TMS

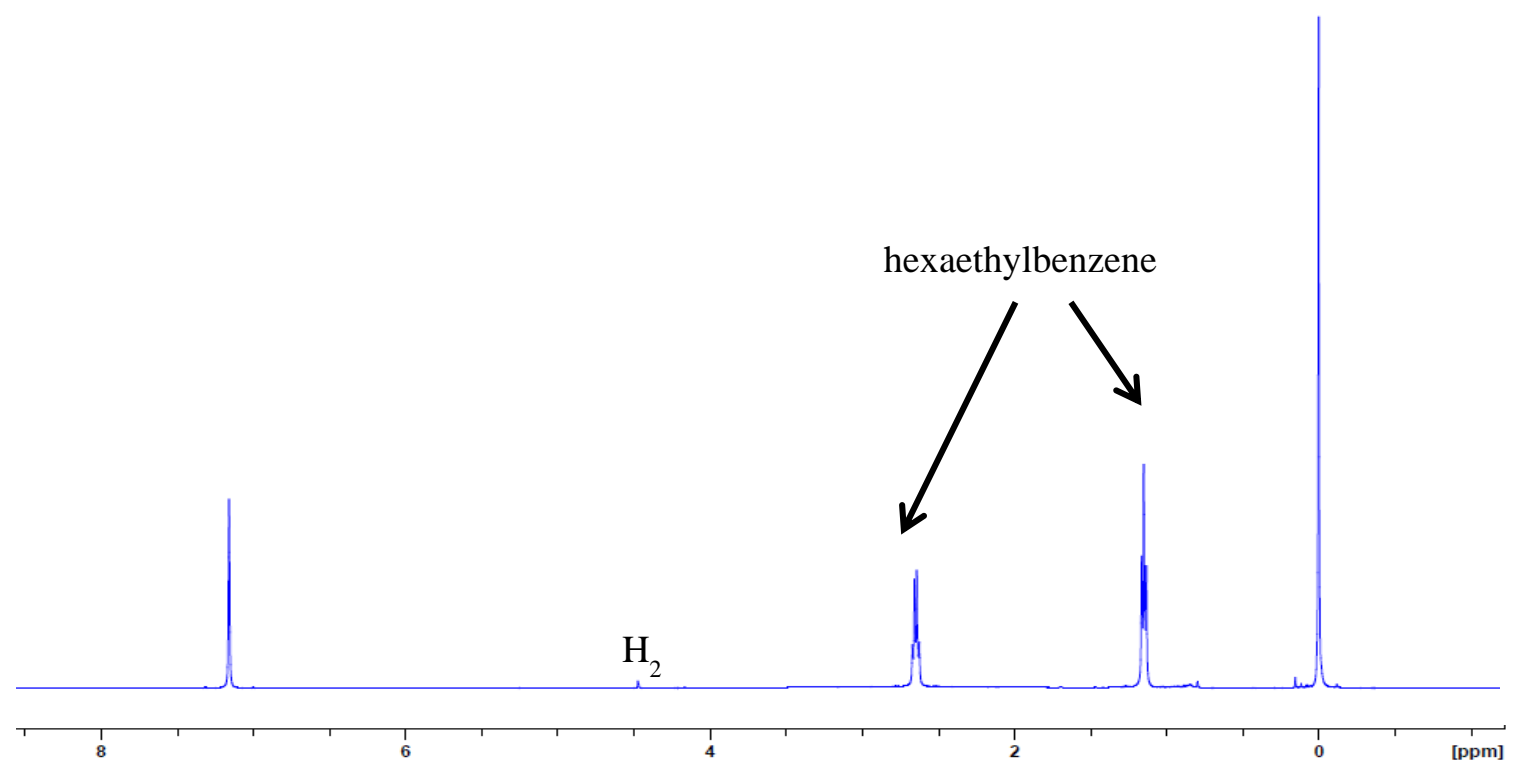

Figure S48: $\quad{ }^{1} \mathrm{H}$ NMR spectrum of $\left[\mathrm{Mn}\left(\mathrm{CH}_{2} \mathrm{SiMe}_{3}\right)_{2}(\mathrm{dmpe})\right](3)$ and $\mathrm{H}_{2}$ after 1 day at $120^{\circ} \mathrm{C}$ in $\mathrm{C}_{6} \mathrm{D}_{6}(500 \mathrm{MHz})$. dmpe signals not observed (presumably broadened due to interactions with paramagnetic metal coating the nmr tube)

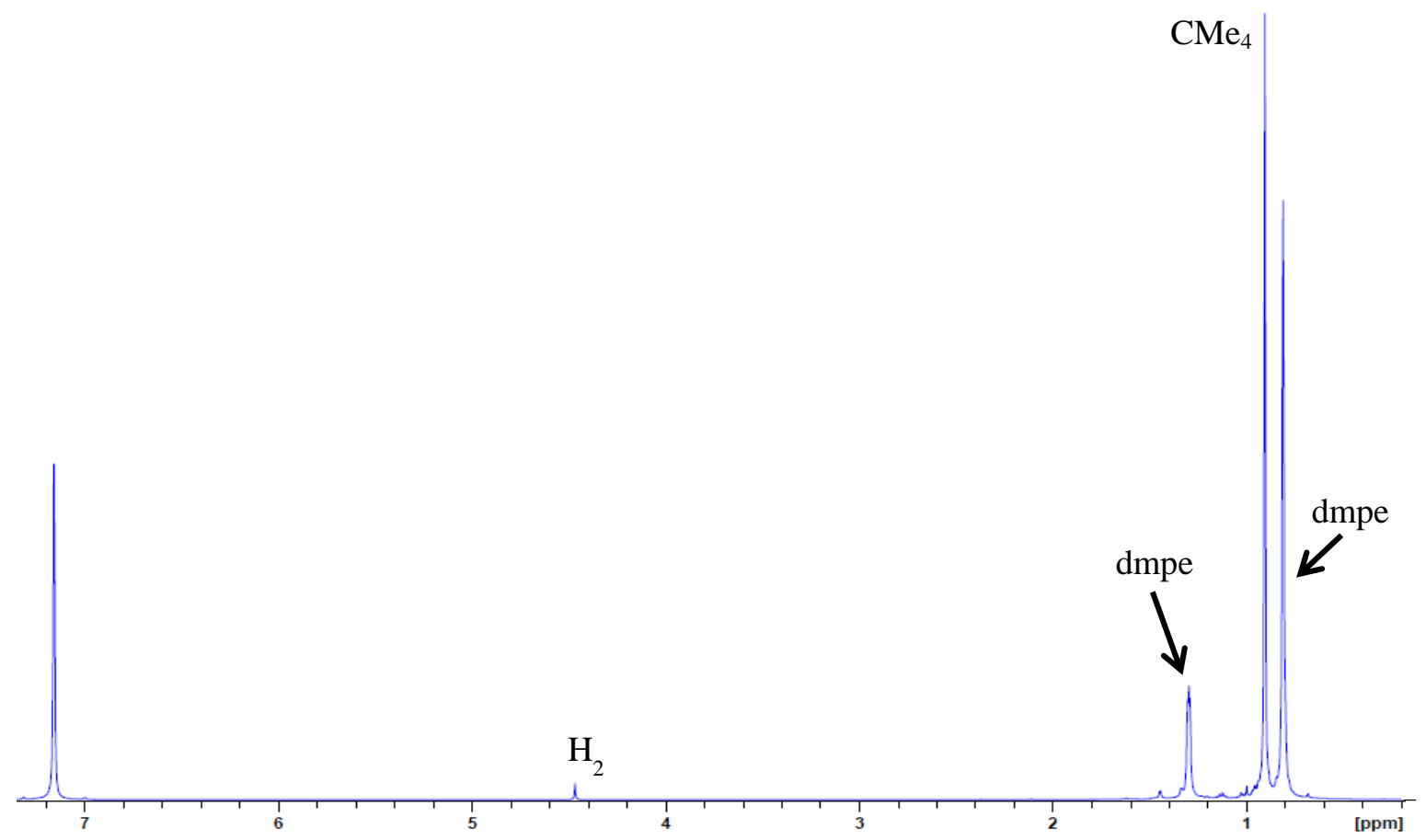

Figure S49: $\quad{ }^{1} \mathrm{H}$ NMR spectrum of $\left[\left\{\mathrm{Mn}\left(\mathrm{CH}_{2} \mathrm{CMe}_{3}\right)_{2}(\mu \text {-dmpe }\}_{2}\right](4)\right.$ and $\mathrm{H}_{2}$ after 1 day at $100^{\circ} \mathrm{C}$ in $\mathrm{C}_{6} \mathbf{D}_{6}(500 \mathrm{MHz})$ 
$\left[\mathrm{Mn}\left(\mathrm{CH}_{2} \mathrm{SiMe}_{3}\right)_{2}(\mathrm{dmpe})\right]$

(3)

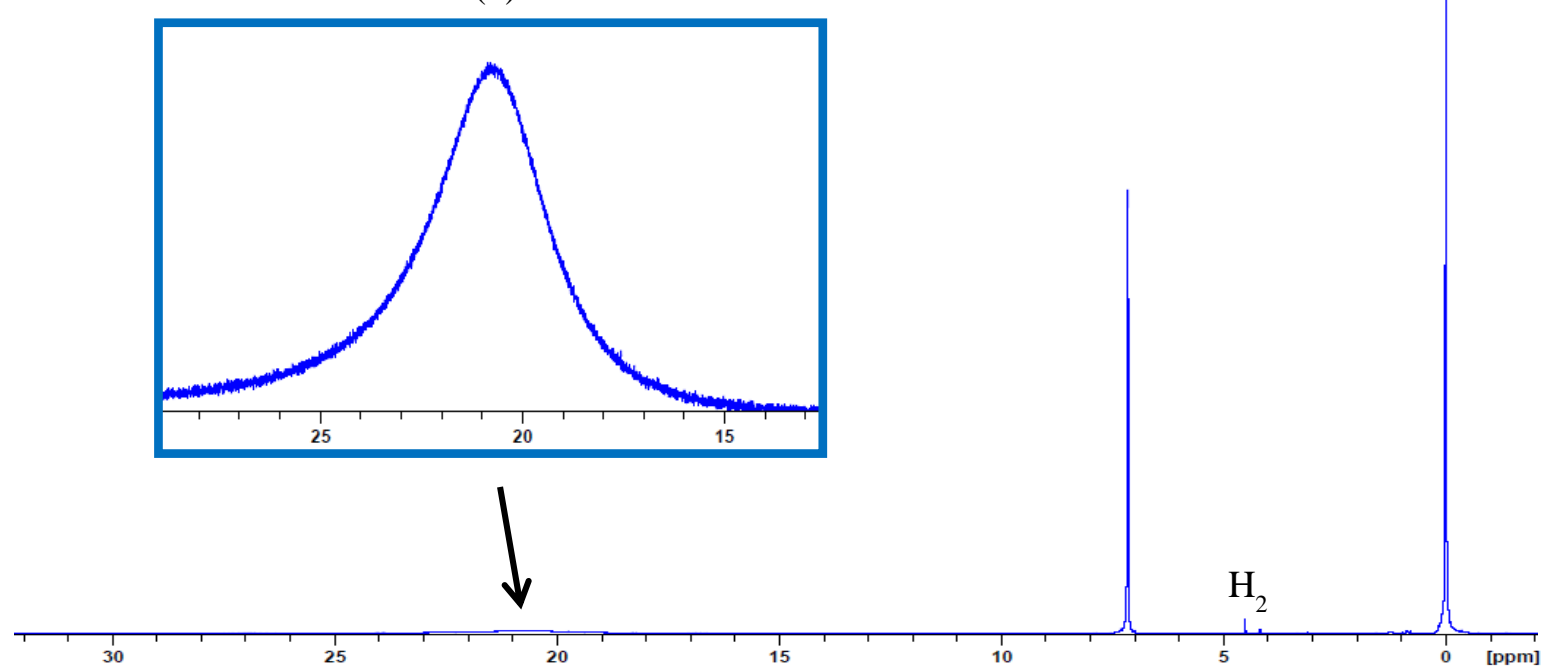

Figure S50: $\quad{ }^{1} \mathrm{H}$ NMR spectrum of $\left[\left\{\mathrm{Mn}\left(\mathrm{CH}_{2} \mathrm{SiMe}_{3}\right)\left(\mu-\mathrm{CH}_{2} \mathrm{SiMe}_{3}\right)\right\}_{2}(\mu\right.$-dmpe $\left.)\right](5)$ and $\mathrm{H}_{2}$ after 4 hours at $120^{\circ} \mathrm{C}$ in $\mathrm{C}_{6} \mathrm{D}_{6}(500 \mathrm{MHz})$

TMS

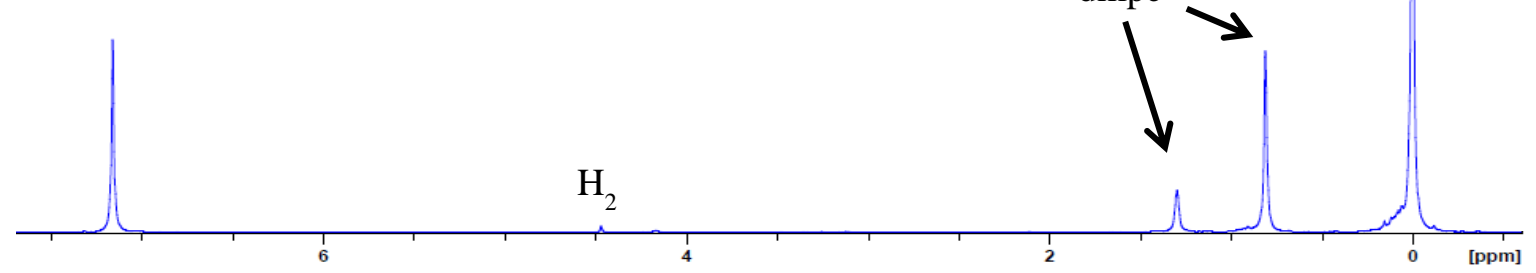

Figure S51: $\quad{ }^{1} \mathrm{H}$ NMR spectrum of $\left[\left\{\mathrm{Mn}\left(\mathrm{CH}_{2} \mathrm{SiMe}_{3}\right)\left(\mu-\mathrm{CH}_{2} \mathrm{SiMe}_{3}\right)\right\}_{2}(\mu-d m p e)\right](5)$ and $\mathrm{H}_{2}$ after 1 day at $120^{\circ} \mathrm{C}$ in $\mathrm{C}_{6} \mathrm{D}_{6}(500 \mathrm{MHz})$ 
$\left[\left\{\mathrm{Mn}\left(\mathrm{CH}_{2} \mathrm{CMe}_{3}\right)_{2}(\mu-\mathrm{dmpe})\right\}_{2}\right]$

$\mathrm{CMe}_{4}$

(4)

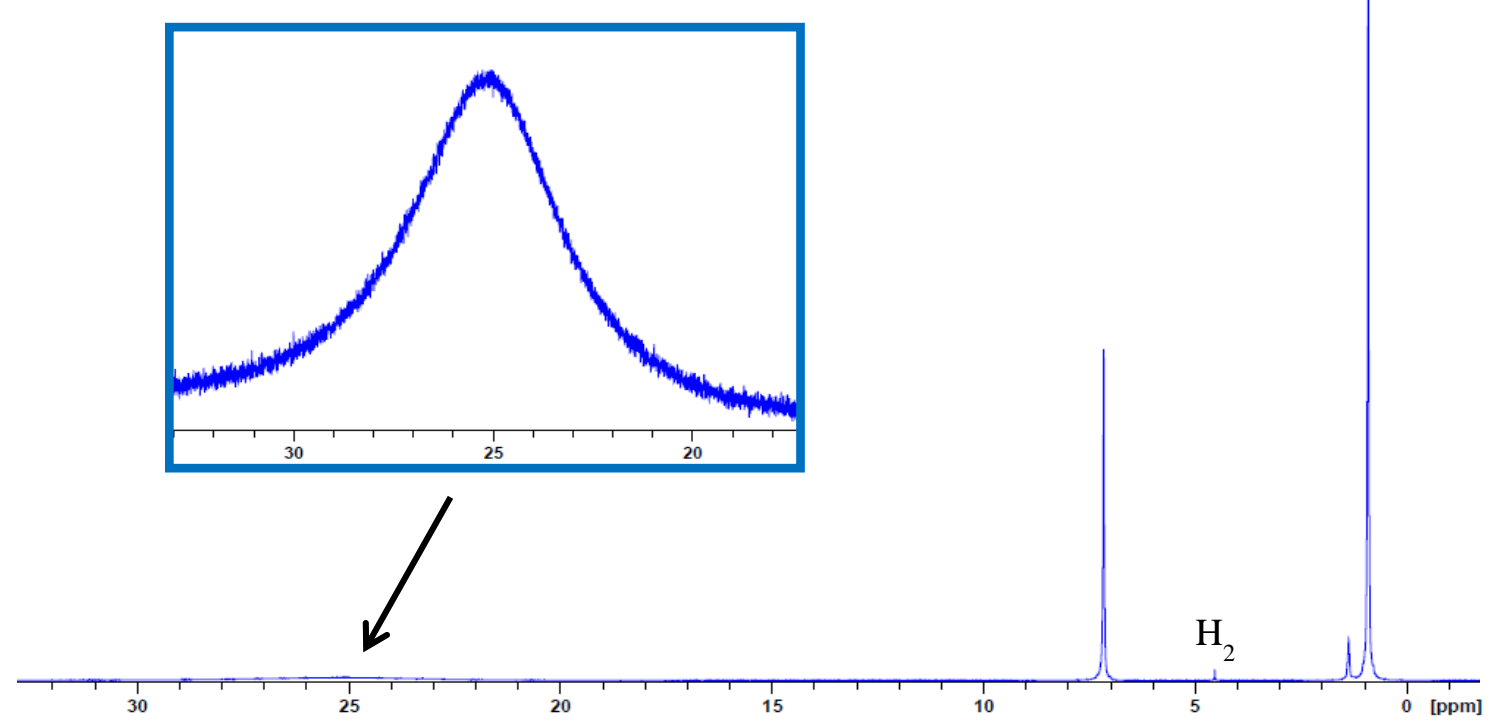

Figure S52: $\quad{ }^{1} \mathrm{H}$ NMR spectrum of $\left[\left\{\mathrm{Mn}\left(\mathrm{CH}_{2} \mathrm{CMe}_{3}\right)\left(\mu-\mathrm{CH}_{2} \mathrm{CMe}_{3}\right)\right\}_{2}(\mu\right.$-dmpe $\left.)\right](6)$ and $\mathrm{H}_{2}$ after 1 hour at $60^{\circ} \mathrm{C}$ in $\mathrm{C}_{6} \mathrm{D}_{6}(500 \mathrm{MHz})$

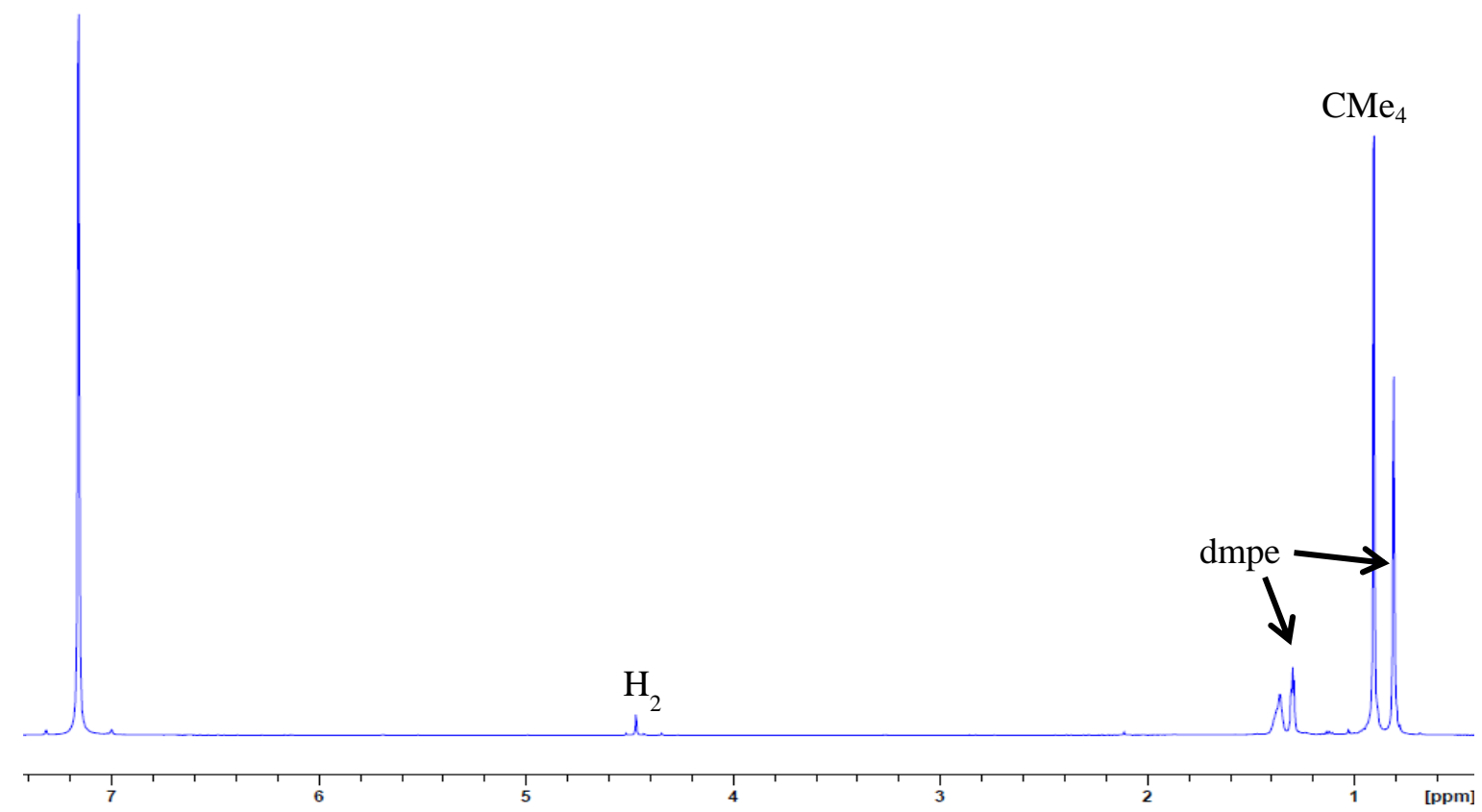

Figure S53: $\quad{ }^{1} \mathrm{H}$ NMR spectrum of $\left[\left\{\mathrm{Mn}\left(\mathrm{CH}_{2} \mathrm{CMe}_{3}\right)\left(\mu-\mathrm{CH}_{2} \mathrm{CMe}_{3}\right)\right\}_{2}(\mu\right.$-dmpe) $](6)$ and $\mathrm{H}_{2}$ after 1 day at $100^{\circ} \mathrm{C}$ in $\mathrm{C}_{6} \mathrm{D}_{6}(500 \mathrm{MHz})$ 


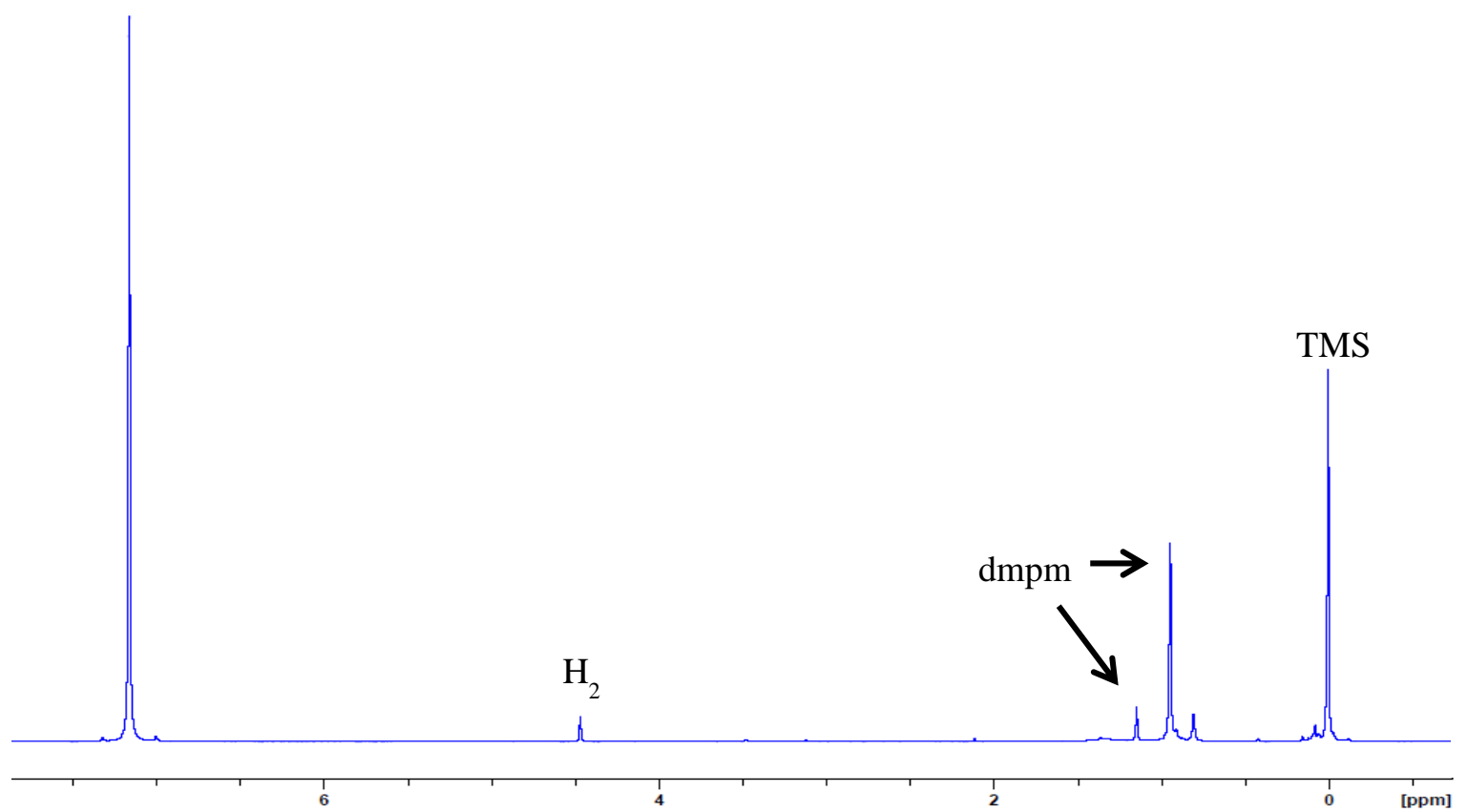

Figure S54: $\quad{ }^{1} \mathrm{H}$ NMR spectrum of $\left[\left\{\mathrm{Mn}\left(\mathrm{CH}_{2} \mathrm{SiMe}_{3}\right)\left(\mu-\mathrm{CH}_{2} \mathrm{SiMe}_{3}\right)\right\}_{2}(\mu\right.$-dmpm) $](7)$ and $\mathrm{H}_{2}$ after 4 hours at $120^{\circ} \mathrm{C}$ in $\mathrm{C}_{6} \mathrm{D}_{6}(500 \mathrm{MHz})$

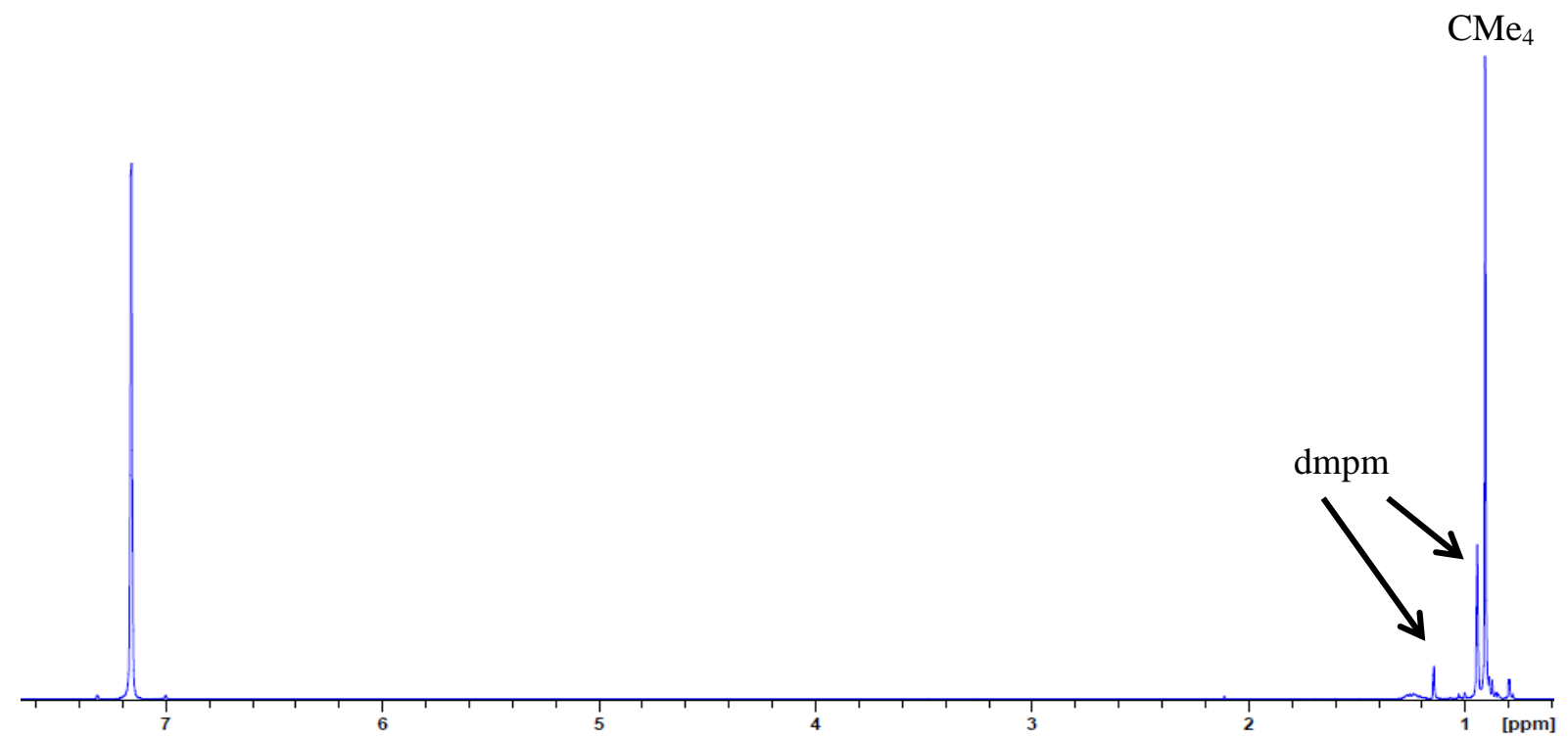

Figure S55: $\quad{ }^{1} \mathrm{H}$ NMR spectrum of $\left[\left\{\mathrm{Mn}\left(\mathrm{CH}_{2} \mathrm{CMe}_{3}\right)\left(\mu-\mathrm{CH}_{2} \mathrm{CMe}_{3}\right)\right\}_{2}(\mu-\mathrm{dmpm})\right](8)$ and $\mathrm{H}_{2}$ after 1 hour at $70^{\circ} \mathrm{C}$ in $\mathrm{C}_{6} \mathrm{D}_{6}(500 \mathrm{MHz})$ 
Figures S56-S63: $\quad{ }^{1} \mathrm{H}$ NMR spectra of results of $\mathrm{ZnEt}_{2}$ reactions with 1 -8

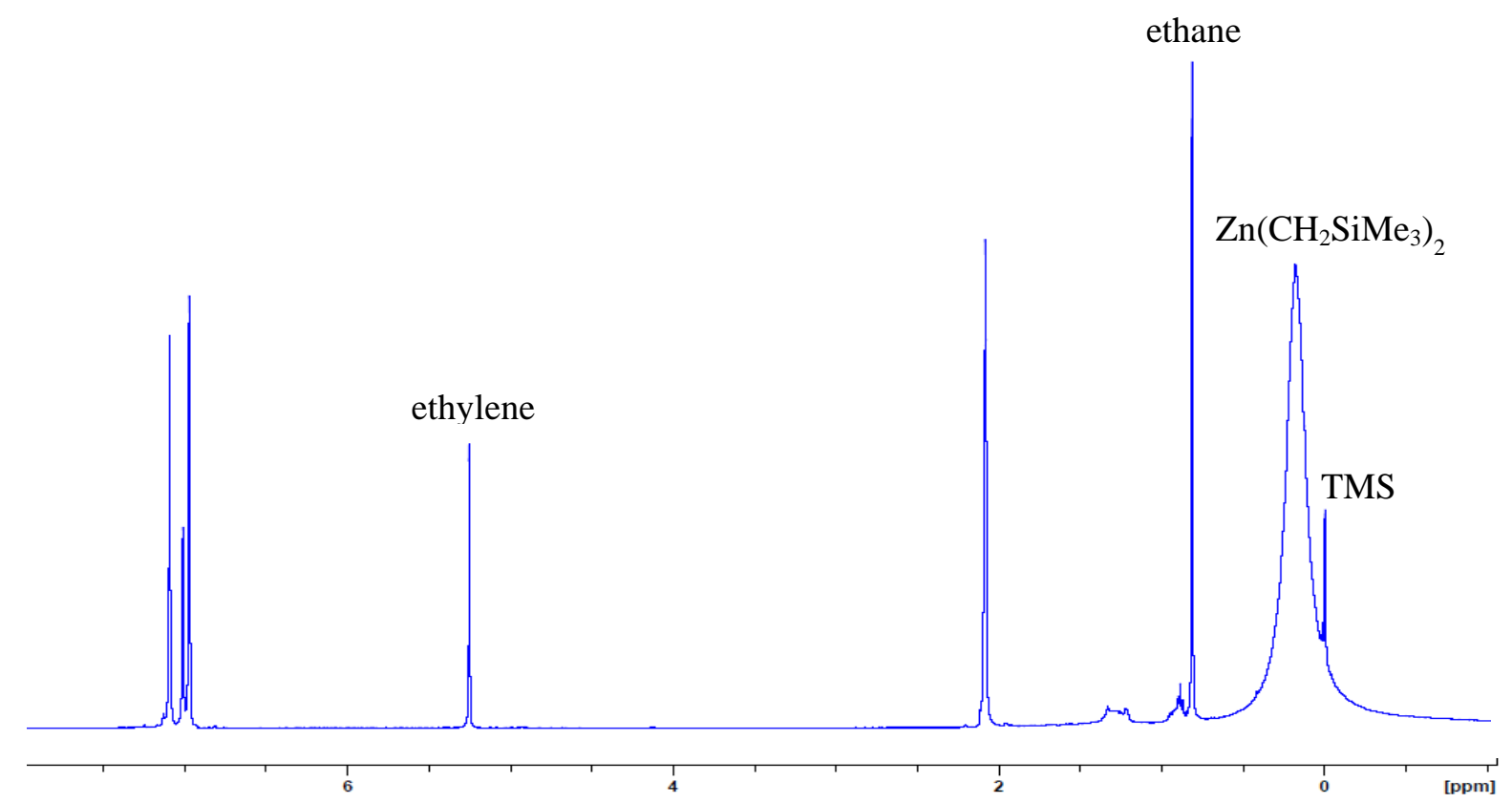

Figure S56: $\quad{ }^{1} \mathrm{H}$ NMR spectrum of $\left[\left\{\mathrm{Mn}\left(\mu-\mathrm{CH}_{2} \mathrm{SiMe}_{3}\right)_{2}\right\}_{\infty}\right](1)$ and $\mathrm{ZnEt}_{2}$ after 1 day at room temperature in $\mathrm{d}_{8}$-touene decanted from the manganese-coated tube $(600 \mathrm{MHz})$

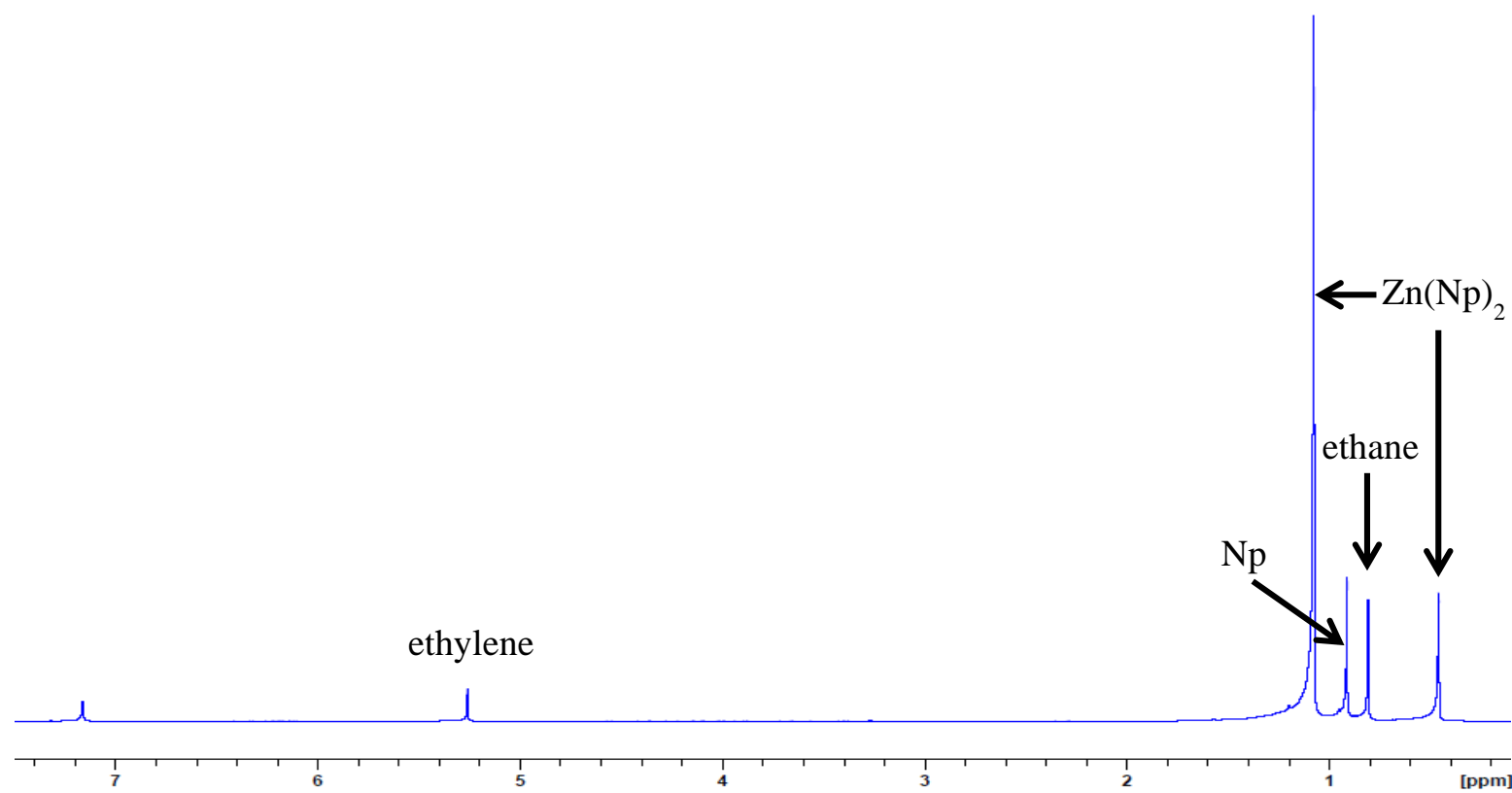

Figure S57: $\quad{ }^{1} \mathrm{H}$ NMR spectrum of $\left[\left\{\mathrm{Mn}\left(\mathrm{CH}_{2} \mathrm{CMe}_{3}\right)\left(\mu-\mathrm{CH}_{2} \mathrm{CMe}_{3}\right)_{2}\right\}_{2}\left\{\mathrm{Mn}\left(\mu-\mathrm{CH}_{2} \mathrm{CMe}_{3}\right)_{2} \mathrm{Mn}\right\}\right]$ (2) and $\mathrm{ZnEt}_{2}$ after 12 hours at room temperature in $\mathrm{C}_{6} \mathrm{D}_{6}(500 \mathrm{MHz})$ 


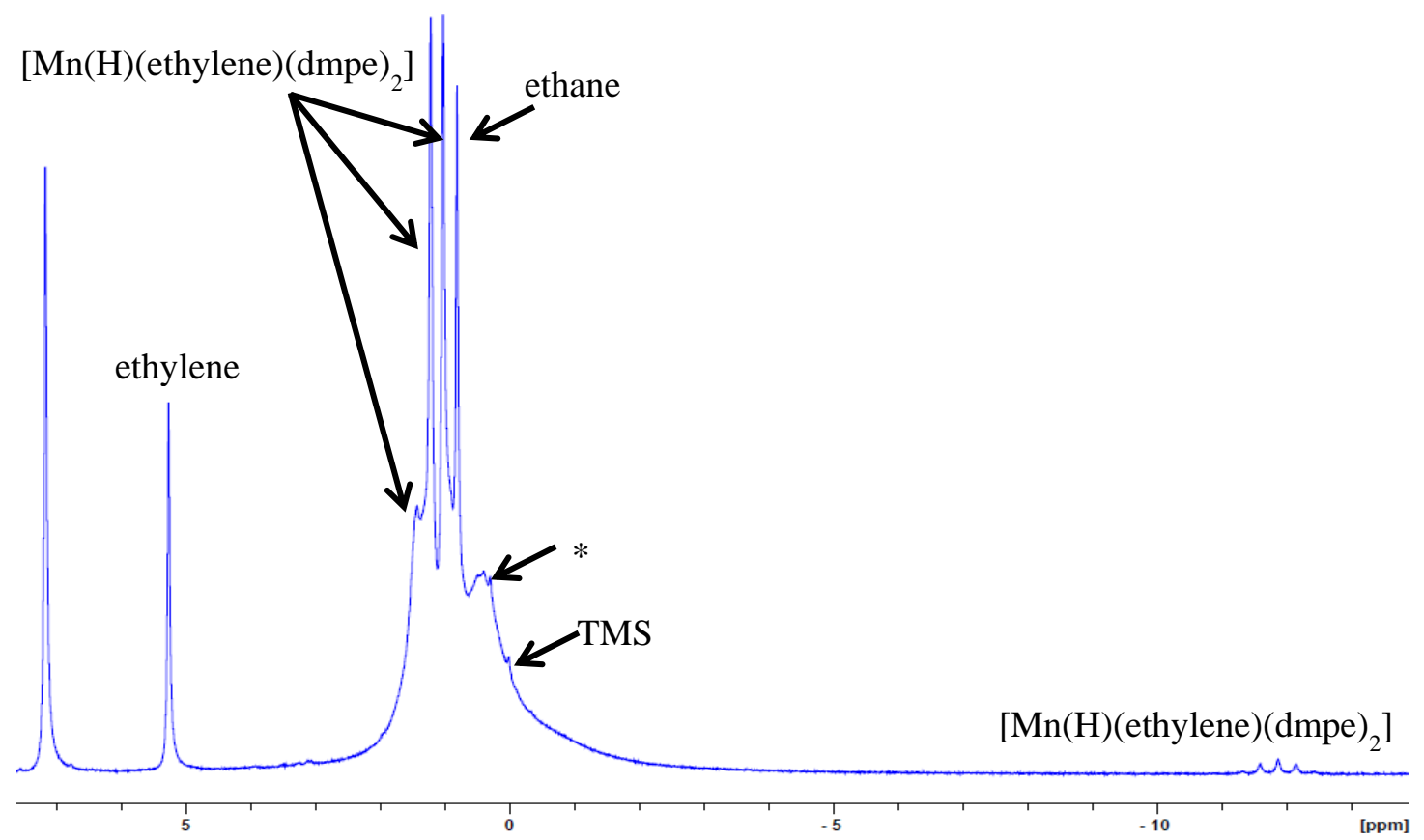

Figure S58: $\quad{ }^{1} \mathrm{H}$ NMR spectrum of $\left[\mathrm{Mn}\left(\mathrm{CH}_{2} \mathrm{SiMe}_{3}\right)_{2}(\mathrm{dmpe})\right](3)$ and $\mathrm{ZnEt}_{2}$ after 1 hour at $60^{\circ} \mathrm{C}$ in $\mathrm{C}_{6} \mathrm{D}_{6}$ * *Presumed to be a mixed alkyl zinc species. $(200 \mathrm{MHz})$

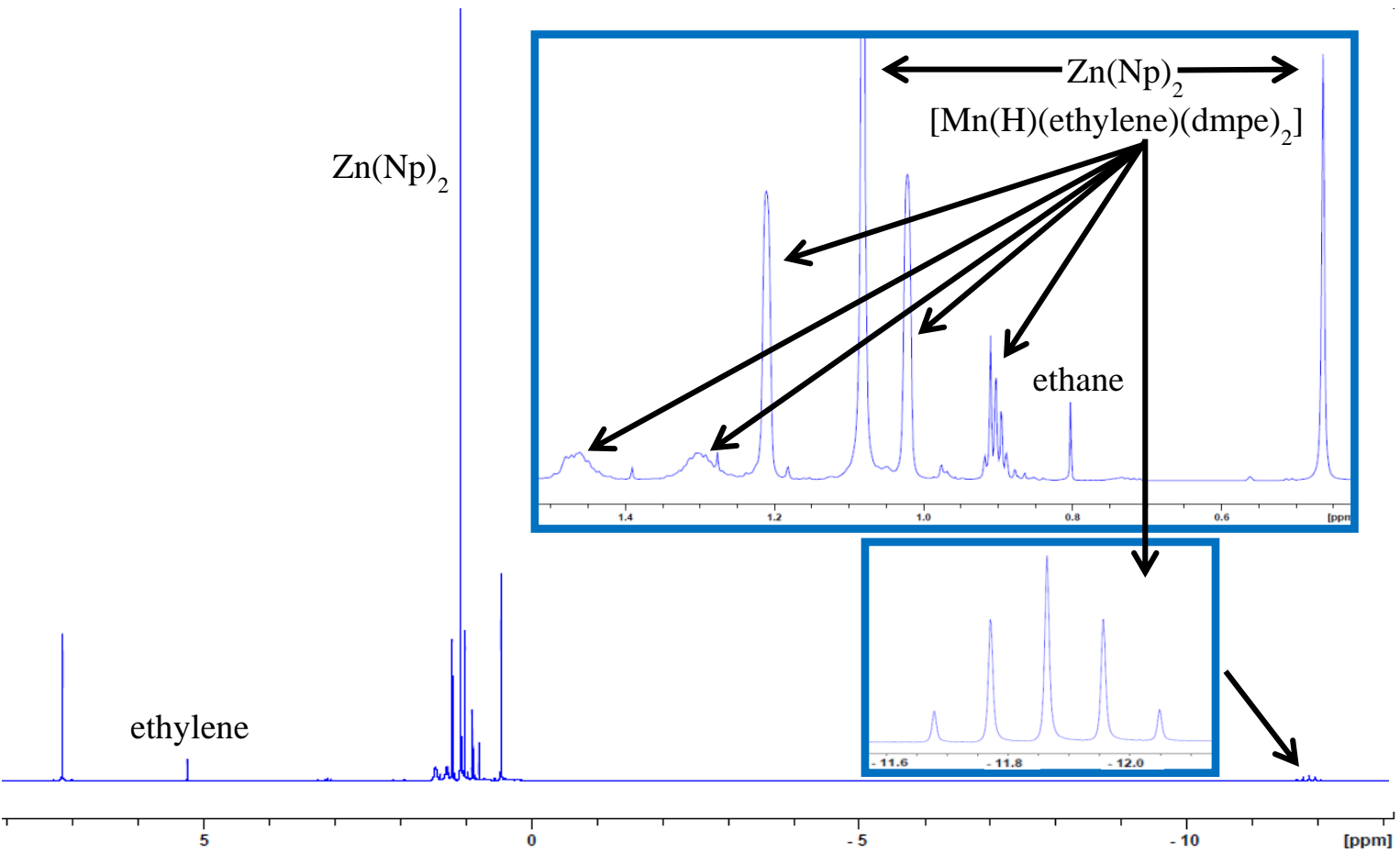

Figure S59: $\quad{ }^{1} \mathrm{H}$ NMR spectrum of $\left[\left\{\mathrm{Mn}\left(\mathrm{CH}_{2} \mathrm{CMe}_{3}\right)_{2}(\mu \text {-dmpe })\right\}_{2}\right](4)$ and $\mathrm{ZnEt}_{2}$ after 3 days at room temperature decanted from the manganese coated tube in $\mathrm{C}_{6} \mathrm{D}_{6}(600 \mathrm{MHz})$ 


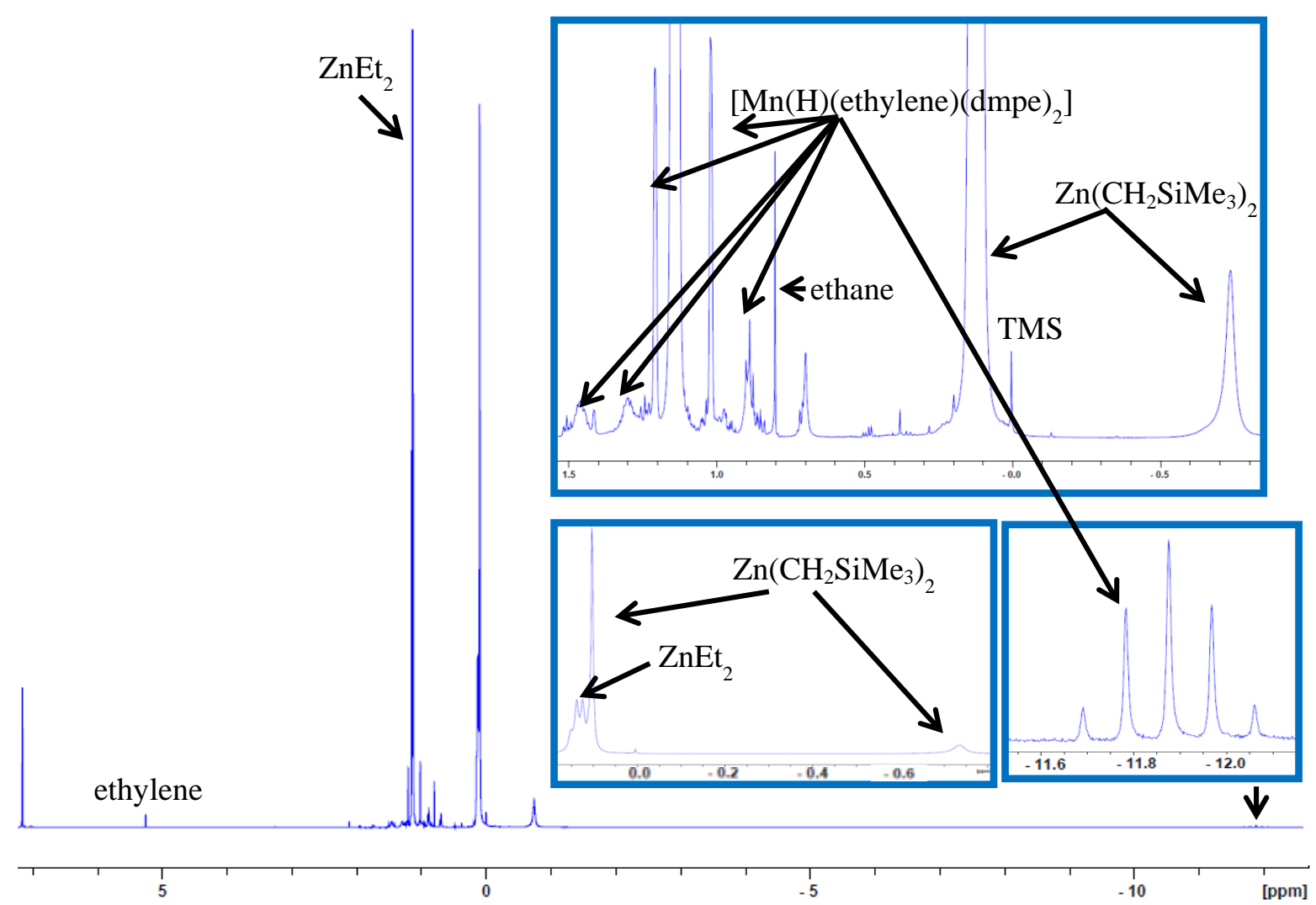

Figure S60: $\quad{ }^{1} \mathrm{H}$ NMR spectrum of $\left[\left\{\mathrm{Mn}\left(\mathrm{CH}_{2} \mathrm{SiMe}_{3}\right)\left(\mu-\mathrm{CH}_{2} \mathrm{SiMe}_{3}\right)\right\}_{2}(\mu\right.$-dmpe $\left.)\right](5)$ and $\mathrm{ZnEt}_{2}$ after 2 days at room temperature decanted from the manganese coated tube in $C_{6} D_{6}(600$ MHz) 


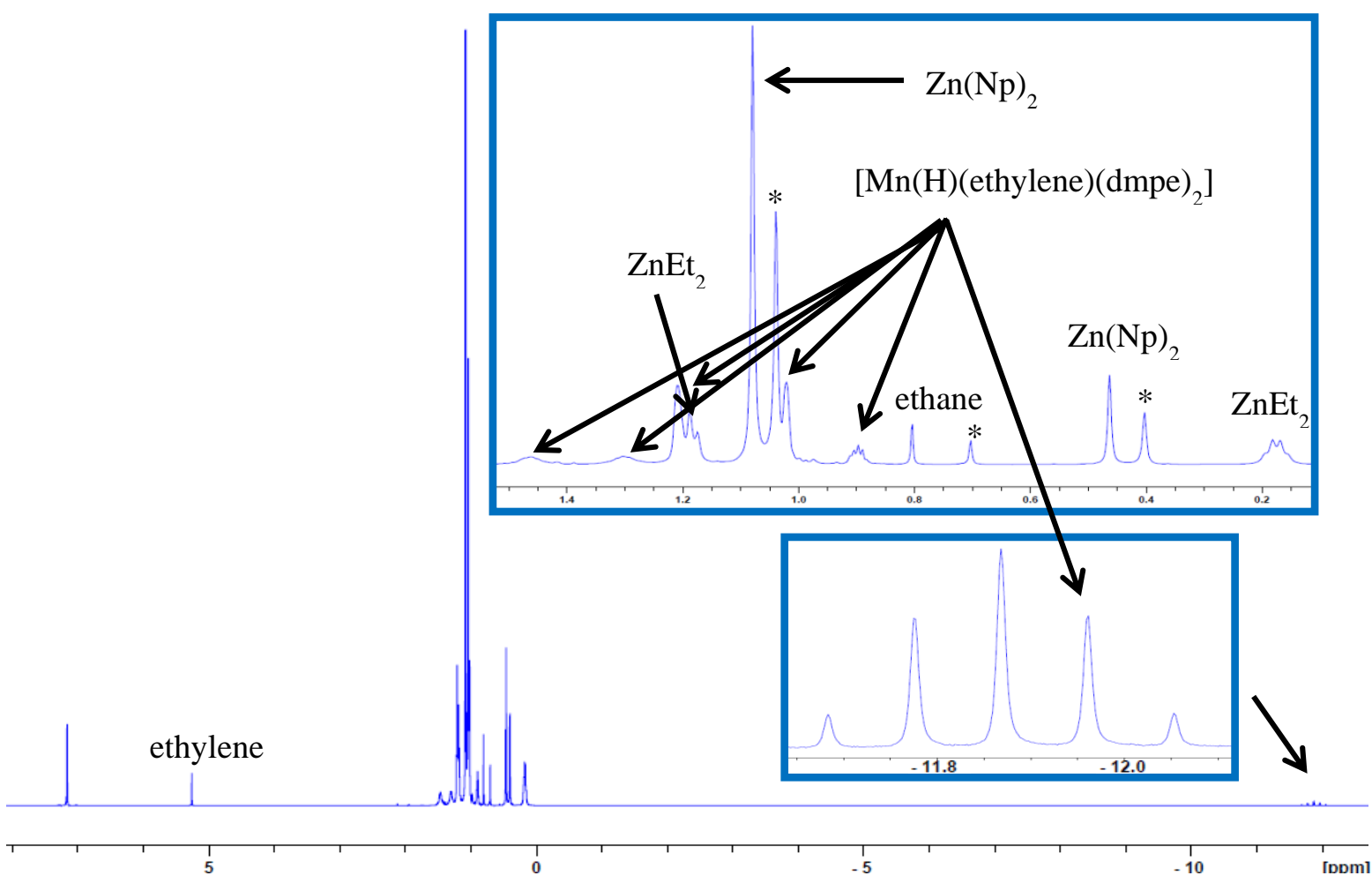

Figure S61: $\quad{ }^{1} \mathrm{H}$ NMR spectrum of $\left[\left\{\mathrm{Mn}\left(\mathrm{CH}_{2} \mathrm{CMe}_{3}\right)\left(\mu-\mathrm{CH}_{2} \mathrm{CMe}_{3}\right)\right\}_{2}(\mu\right.$-dmpe $\left.)\right](6)$ and $\mathrm{ZnEt}_{2}$ after 12 hours at room temperature decanted from the manganese coated tube in $\mathbf{C}_{6} \mathbf{D}_{6}$ $(600 \mathrm{MHz}) . *$ is presumed to be from a mixed alkyl-zinc phosphine containing byproduct 


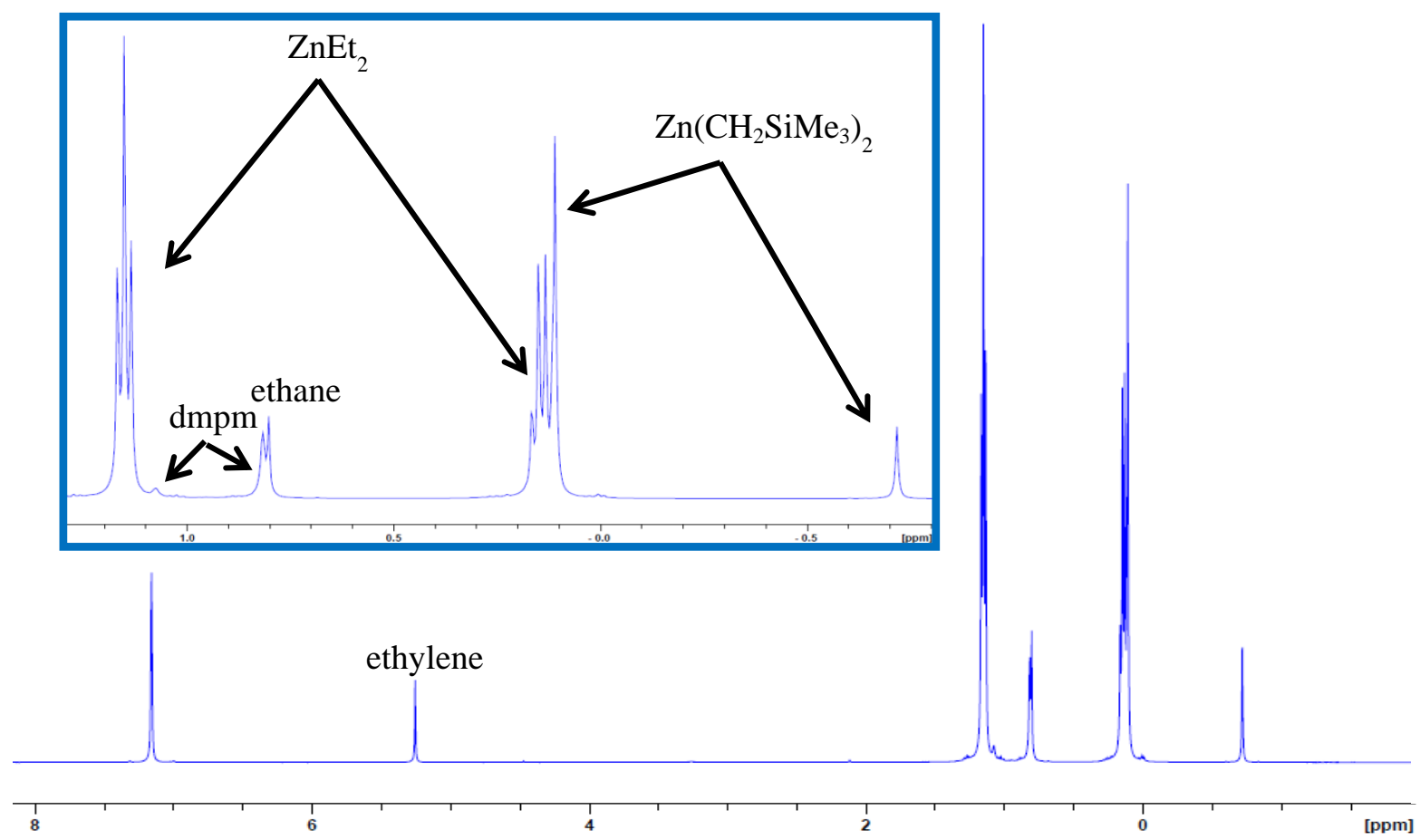

Figure S62: ${ }^{1} \mathrm{H}$ NMR spectrum of $\left[\left\{\mathrm{Mn}\left(\mathrm{CH}_{2} \mathrm{SiMe}_{3}\right)\left(\mu-\mathrm{CH}_{2} \mathrm{SiMe}_{3}\right)\right\}_{2}(\mu-\mathrm{dmpm})\right](7)$ and $\mathrm{ZnEt}_{2}$ after 1.5 hours at $90^{\circ} \mathrm{C}$ in $\mathrm{C}_{6} \mathrm{D}_{6}(500 \mathrm{MHz})$

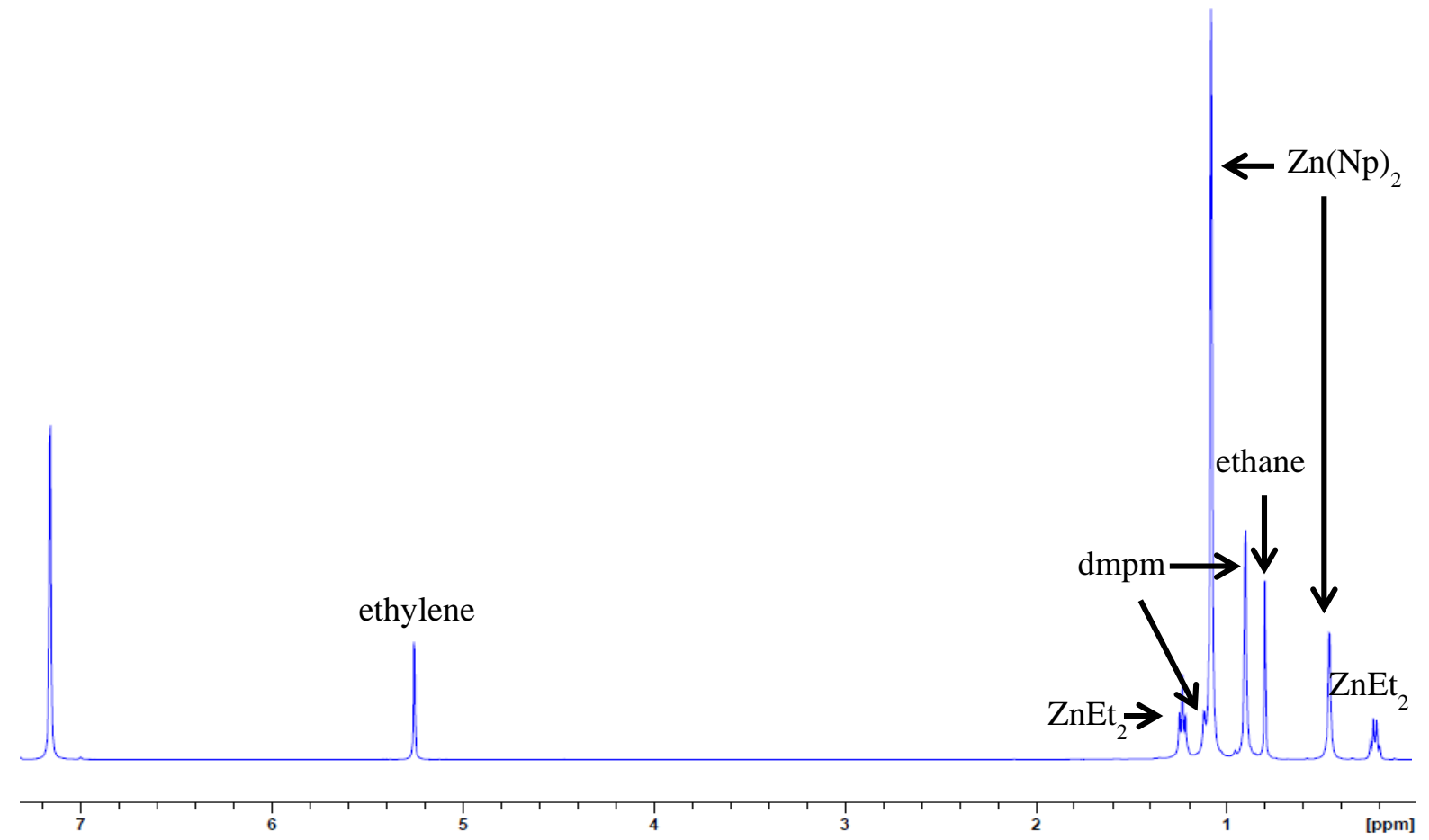

Figure S63:

${ }^{1} \mathrm{H}$ NMR spectrum of $\left[\left\{\mathrm{Mn}\left(\mathrm{CH}_{2} \mathrm{CMe}_{3}\right)\left(\mu-\mathrm{CH}_{2} \mathrm{CMe}_{3}\right)\right\}_{2}(\mu-\mathrm{dmpm})\right](8)$ and $\mathrm{ZnEt}_{2}$ after 1.5 hours at $90^{\circ} \mathrm{C}$ in $\mathrm{C}_{6} \mathrm{D}_{6}(500 \mathrm{MHz})$ 


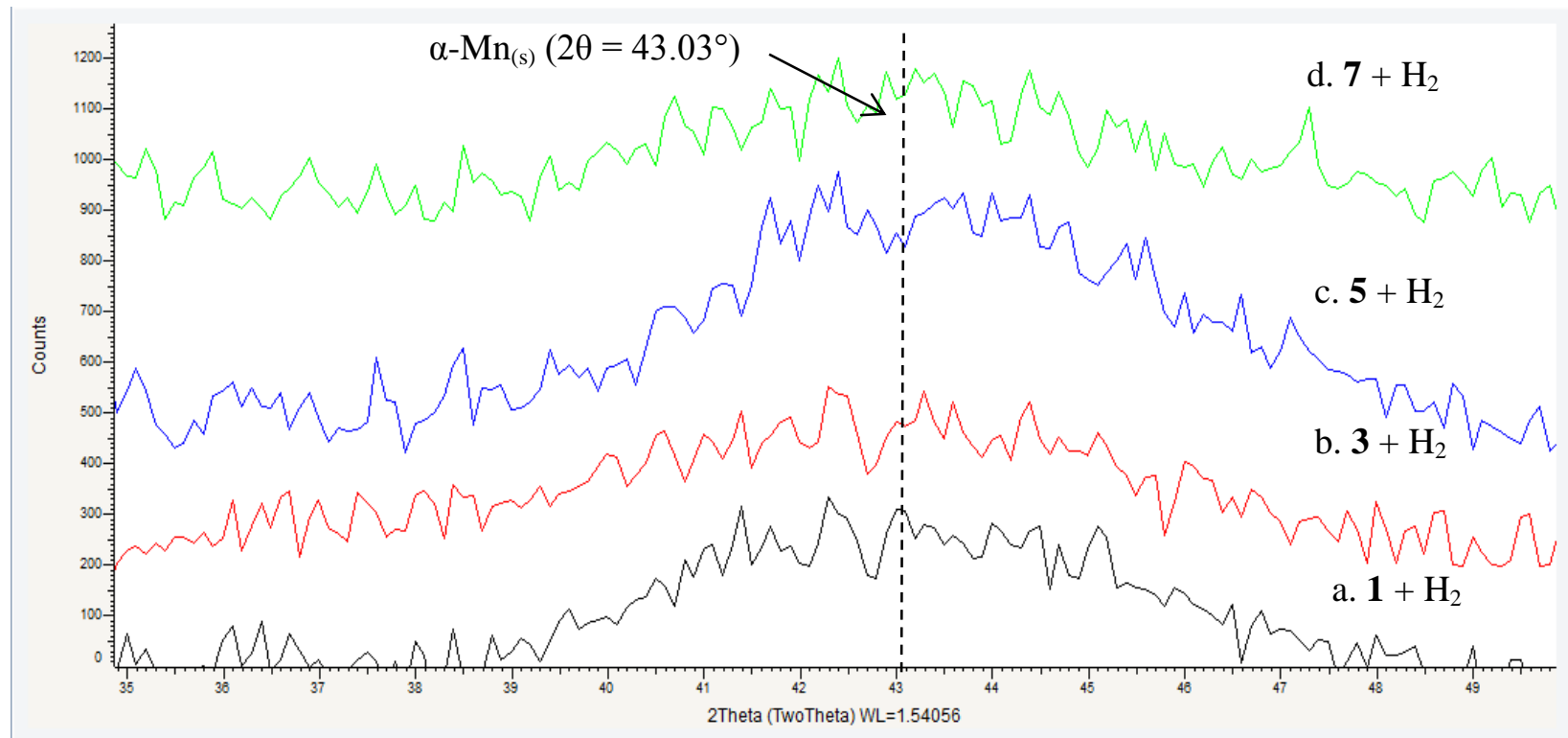

Figure S64: 2D Powder X-ray diffractograms (300 K) of metallic-looking mirrors deposited by reactions of $\mathrm{H}_{2}$ with a. $\left[\left\{\mathrm{Mn}\left(\mu-\mathrm{CH}_{2} \mathrm{SiMe}_{3}\right)_{2}\right\}_{\infty}\right](1)$, b. $\left[\mathrm{Mn}\left(\mathrm{CH}_{2} \mathrm{SiMe}_{3}\right)_{2}(\mathrm{dmpe})\right]$ (3), c. $\left[\left\{\mathrm{Mn}\left(\mathrm{CH}_{2} \mathrm{SiMe}_{3}\right)\left(\mu-\mathrm{CH}_{2} \mathrm{SiMe}_{3}\right)\right\}_{2}(\mu-d m p e)\right](5)$, and d. $\left[\left\{\mathrm{Mn}\left(\mathrm{CH}_{2} \mathrm{SiMe}_{3}\right)(\mu-\right.\right.$ $\left.\left.\left.\mathrm{CH}_{2} \mathrm{SiMe}_{3}\right)\right\}_{2}(\mu-\mathrm{dmpm})\right](7)(\mathrm{Cu}$ source $\lambda=1.54056 \AA)$

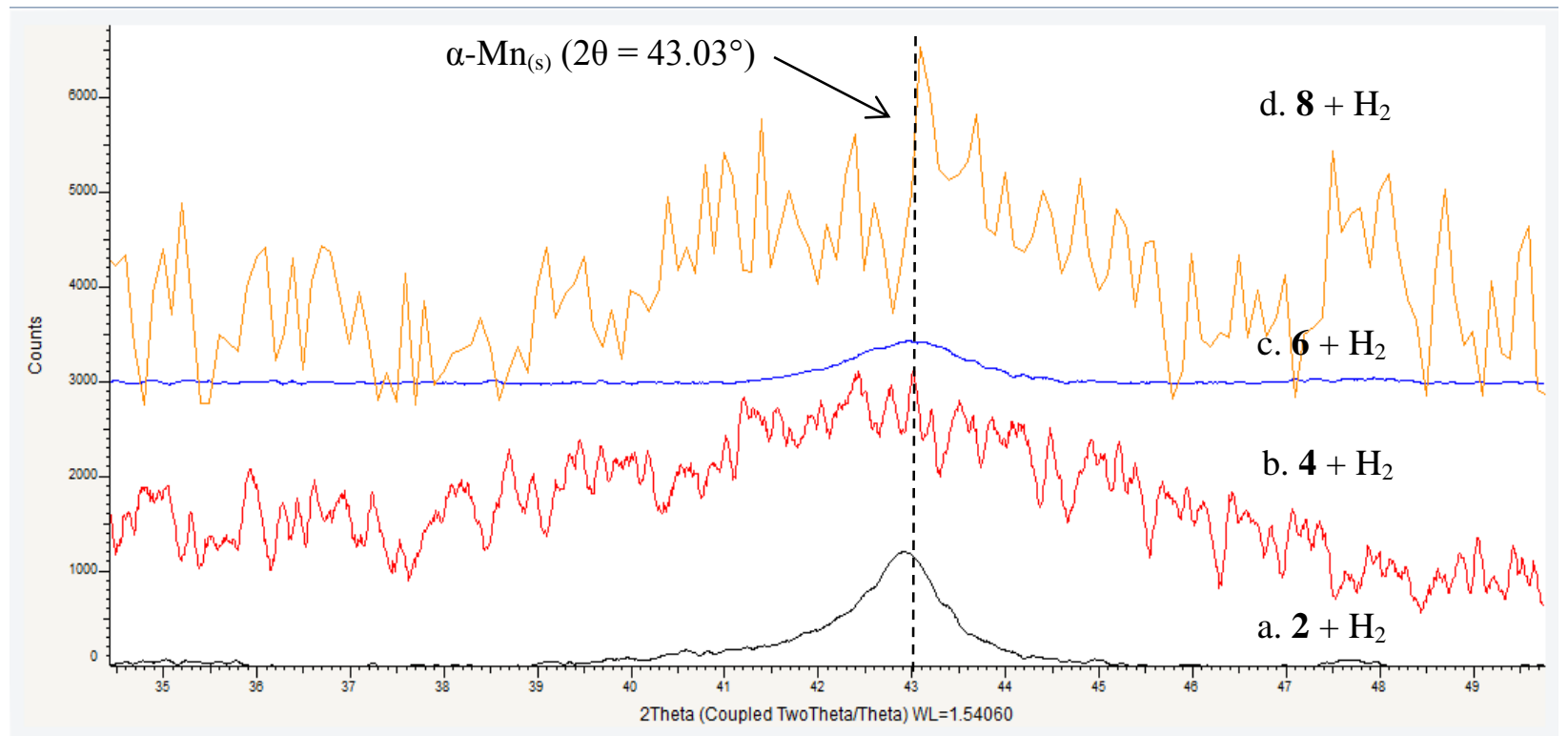

Figure S65: 2D Powder X-ray diffractograms (300 K) of metallic-looking mirrors deposited by reactions of $\mathrm{H}_{2}$ with a. [ $\left.\left.\left\{\mathrm{Mn}\left(\mathrm{CH}_{2} \mathrm{CMe}_{3}\right)\left(\mu-\mathrm{CH}_{2} \mathrm{CMe}_{3}\right)_{2}\right\}_{2}\left\{\mathrm{Mn}\left(\mu-\mathrm{CH}_{2} \mathrm{CMe}_{3}\right)_{2} \mathrm{Mn}\right\}\right]\right]$ (2), b. $\left[\left\{\mathrm{Mn}\left(\mathrm{CH}_{2} \mathrm{CMe}_{3}\right)_{2}(\mu \text {-dmpe })\right\}_{2}\right](4)$, c. $\left[\left\{\mathrm{Mn}\left(\mathrm{CH}_{2} \mathrm{CMe}_{3}\right)\left(\mu-\mathrm{CH}_{2} \mathrm{CMe}_{3}\right)\right\}_{2}(\mu\right.$-dmpe) $(6)$, and d. $\left[\left\{\mathrm{Mn}\left(\mathrm{CH}_{2} \mathrm{CMe}_{3}\right)\left(\mu-\mathrm{CH}_{2} \mathrm{CMe}_{3}\right)\right\}_{2}(\mu-\mathrm{dmpm})\right](8)(\mathrm{Cu}$ source $\lambda=1.54056 \AA)$ 


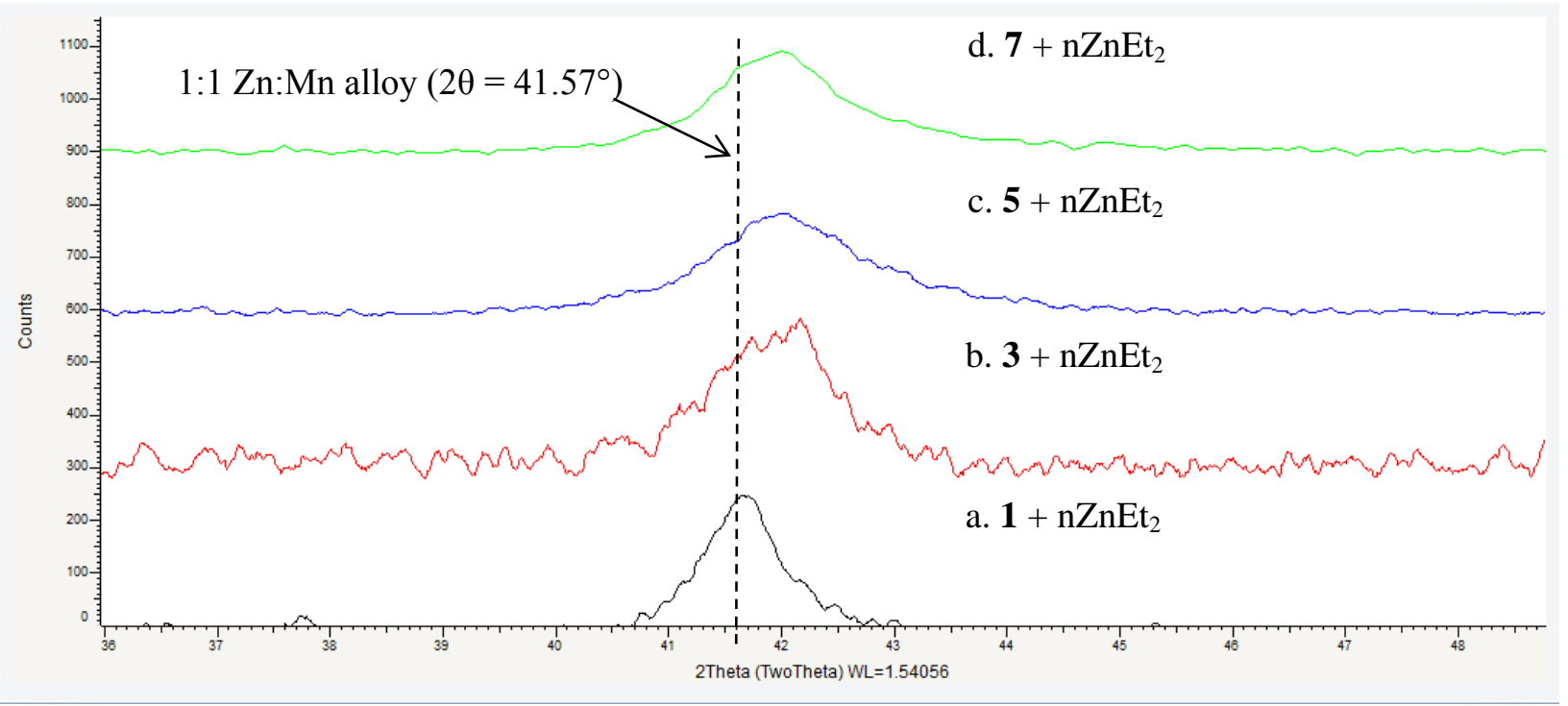

Figure S66: 2D Powder X-ray diffractograms (300 K) of metallic-looking mirrors deposited by reactions of $\mathrm{ZnEt}_{2}$ with a. $\left[\left\{\mathrm{Mn}\left(\mu-\mathrm{CH}_{2} \mathrm{SiMe}_{3}\right)_{2}\right\}_{\infty}\right](1)$, b. $\left[\mathrm{Mn}\left(\mathrm{CH}_{2} \mathrm{SiMe}_{3}\right)_{2}(\mathrm{dmpe})\right]$ (3), c. $\left[\left\{\mathrm{Mn}\left(\mathrm{CH}_{2} \mathrm{SiMe}_{3}\right)\left(\mu-\mathrm{CH}_{2} \mathrm{SiMe}_{3}\right)\right\}_{2}(\mu-\mathrm{dmpe})\right](5)$, and d. $\left[\left\{\mathrm{Mn}\left(\mathrm{CH}_{2} \mathrm{SiMe}_{3}\right)(\mu-\right.\right.$ $\left.\left.\left.\mathrm{CH}_{2} \mathrm{SiMe}_{3}\right)\right\}_{2}(\mu-\mathrm{dmpm})\right](7)(\mathrm{Cu}$ source $\lambda=1.54056 \AA)$

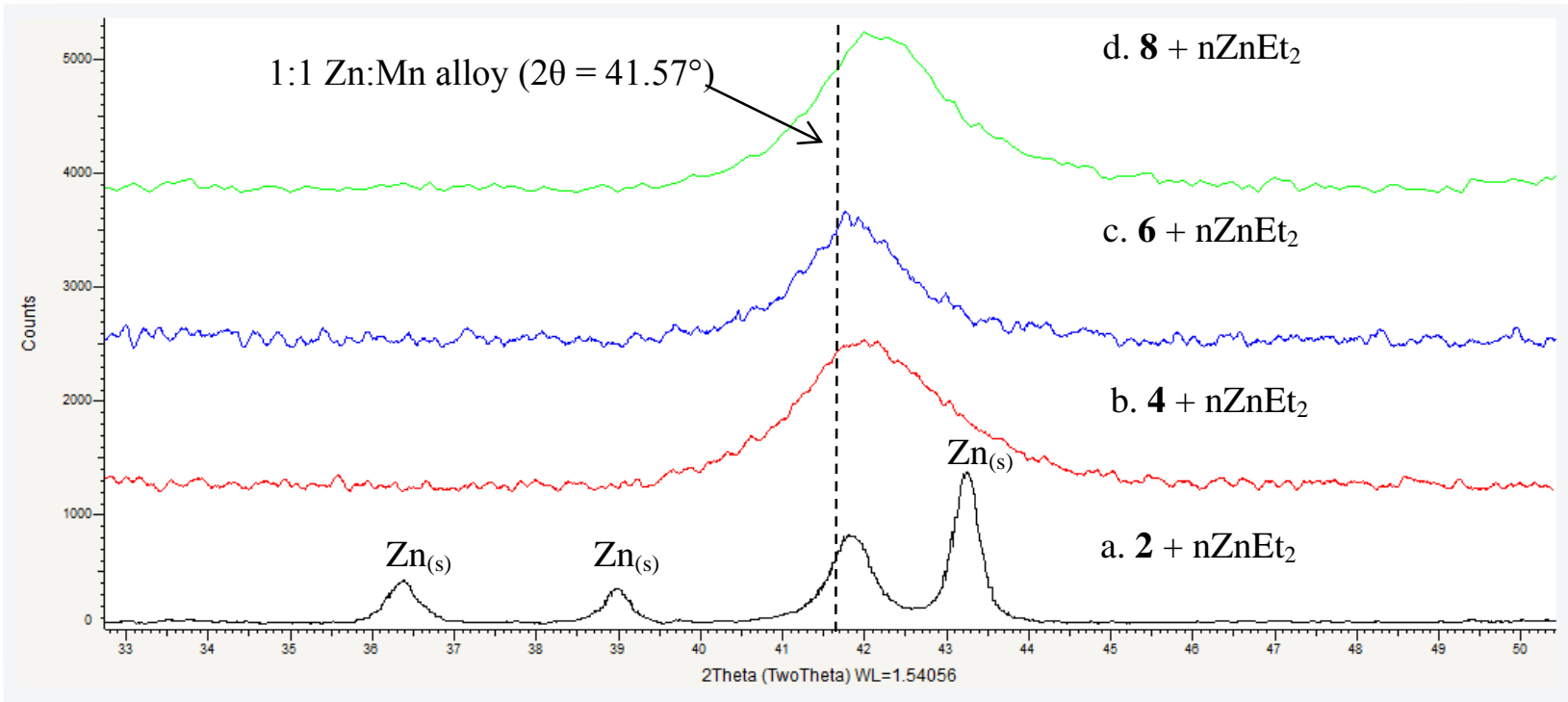

Figure S67: 2D Powder X-ray diffractograms (300 K) of metallic-looking mirrors deposited by reactions of $\mathrm{ZnEt}_{2}$ with a. $\left[\left\{\mathrm{Mn}\left(\mathrm{CH}_{2} \mathrm{CMe}_{3}\right)\left(\mu-\mathrm{CH}_{2} \mathrm{CMe}_{3}\right)_{2}\right\}_{2}\left\{\mathrm{Mn}\left(\mu-\mathrm{CH}_{2} \mathrm{CMe}_{3}\right)_{2} \mathrm{Mn}\right\}\right]$ (2), b. $\left[\left\{\mathrm{Mn}\left(\mathrm{CH}_{2} \mathrm{CMe}_{3}\right)_{2}(\mu \text {-dmpe })\right\}_{2}\right](4)$, c. $\left[\left\{\mathrm{Mn}\left(\mathrm{CH}_{2} \mathrm{CMe}_{3}\right)\left(\mu-\mathrm{CH}_{2} \mathrm{CMe}_{3}\right)\right\}_{2}(\mu-d m p e)\right]$ (6), and d. $\left[\left\{\mathrm{Mn}\left(\mathrm{CH}_{2} \mathrm{CMe}_{3}\right)\left(\mu-\mathrm{CH}_{2} \mathrm{CMe}_{3}\right)\right\}_{2}(\mu-\mathrm{dmpm})\right]$ (8) $(\mathrm{Cu}$ source $\lambda=1.54056$ A) 
Figures S68-S71: $\quad$ XPS of powders deposited by $\underline{\mathrm{ZnEt}}_{2} \underline{\text { reactions with } 2 \text { and } 7}$
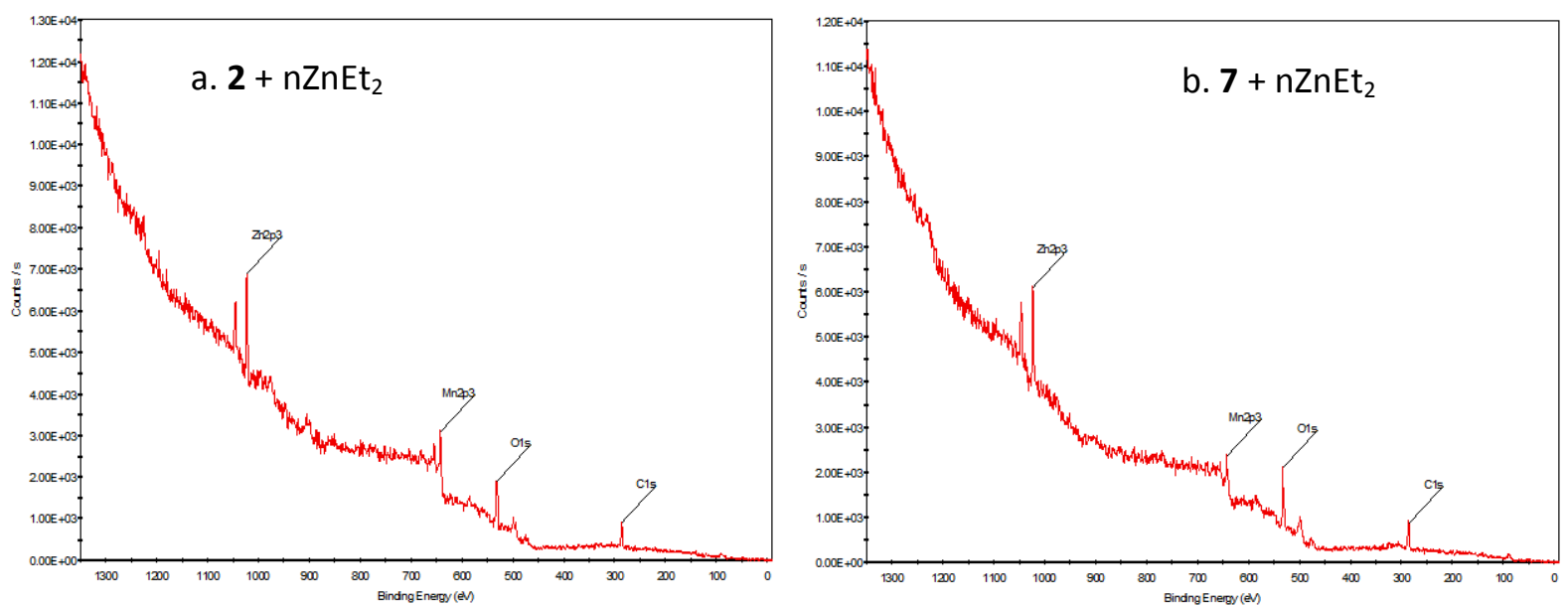

Figure S68: XPS survey scans for mirrors deposited by the reactions of $\mathrm{ZnEt}_{2}$ with a. $\left[\left\{\mathrm{Mn}\left(\mathrm{CH}_{2} \mathrm{CMe}_{3}\right)\left(\mu-\mathrm{CH}_{2} \mathrm{CMe}_{3}\right)_{2}\right\}_{2}\left\{\mathrm{Mn}\left(\mu-\mathrm{CH}_{2} \mathrm{CMe}_{3}\right)_{2} \mathrm{Mn}\right\}\right] \quad$ (2) and b. $\left[\left\{\mathrm{Mn}\left(\mathrm{CH}_{2} \mathrm{SiMe}_{3}\right)\left(\mu-\mathrm{CH}_{2} \mathrm{SiMe}_{3}\right)\right\}_{2}(\mu-\mathrm{dmpm})\right](7)$ (5 scans, $5 \mathrm{~m} 40.3 \mathrm{~s}, 400 \mu \mathrm{m}, \mathrm{CAE}$ 200.0)

a. $\left[\left\{\mathrm{Mn}\left(\mathrm{CH}_{2} \mathrm{CMe}_{3}\right)\left(\mu-\mathrm{CH}_{2} \mathrm{CMe}_{3}\right)_{2}\right\}_{2}\left\{\mathrm{Mn}\left(\mu-\mathrm{CH}_{2} \mathrm{CMe}_{3}\right)_{2} \mathrm{Mn}\right\}\right](2)+\mathrm{nZnEt}_{2}$

\begin{tabular}{|l|r|r|r|r|r|r|}
\hline Name & Peak KE & FWHM eV & Area (P) CPS.eV & Atomic \% & Q & SF Al Scof \\
\hline C1s & 1201.07 & 1.23 & 331.81 & 50.87 & 1 & 1.000 \\
\hline O1s & 954.43 & 1.47 & 581.03 & 32.08 & 1 & 2.930 \\
\hline Mn2p3 & 845.32 & 3.10 & 557.04 & 10.12 & 1 & 9.170 \\
\hline Zn2p3 & 464.34 & 1.32 & 670.92 & 6.92 & 1 & 18.920 \\
\hline
\end{tabular}

b. $\left[\left\{\mathrm{Mn}\left(\mathrm{CH}_{2} \mathrm{SiMe}_{3}\right)\left(\mu-\mathrm{CH}_{2} \mathrm{SiMe}_{3}\right)\right\}_{2}(\mu-\mathrm{dmpm})\right](7)+\mathrm{nZnEt}_{2}$

\begin{tabular}{|l|r|r|r|r|r|r|}
\hline Name & Peak KE & FWHM eV & Area (P) CPS.eV & Atomic \% & $\boldsymbol{Q}$ & SF Al Scof \\
\hline C1s & 1200.90 & 1.50 & 408.87 & 60.96 & 1 & 1.000 \\
\hline O1s & 954.37 & 1.90 & 506.20 & 27.18 & 1 & 2.930 \\
\hline Zn2p3 & 463.98 & 1.45 & 672.25 & 6.75 & 1 & 18.920 \\
\hline Mn2p3 & 844.94 & 3.11 & 289.22 & 5.11 & 1 & 9.170 \\
\hline
\end{tabular}

Figure S69: XPS elemental quantification for mirrors deposited by the reactions of $\mathrm{ZnEt}_{2}$ with a. compound 2 and b. compound 7 

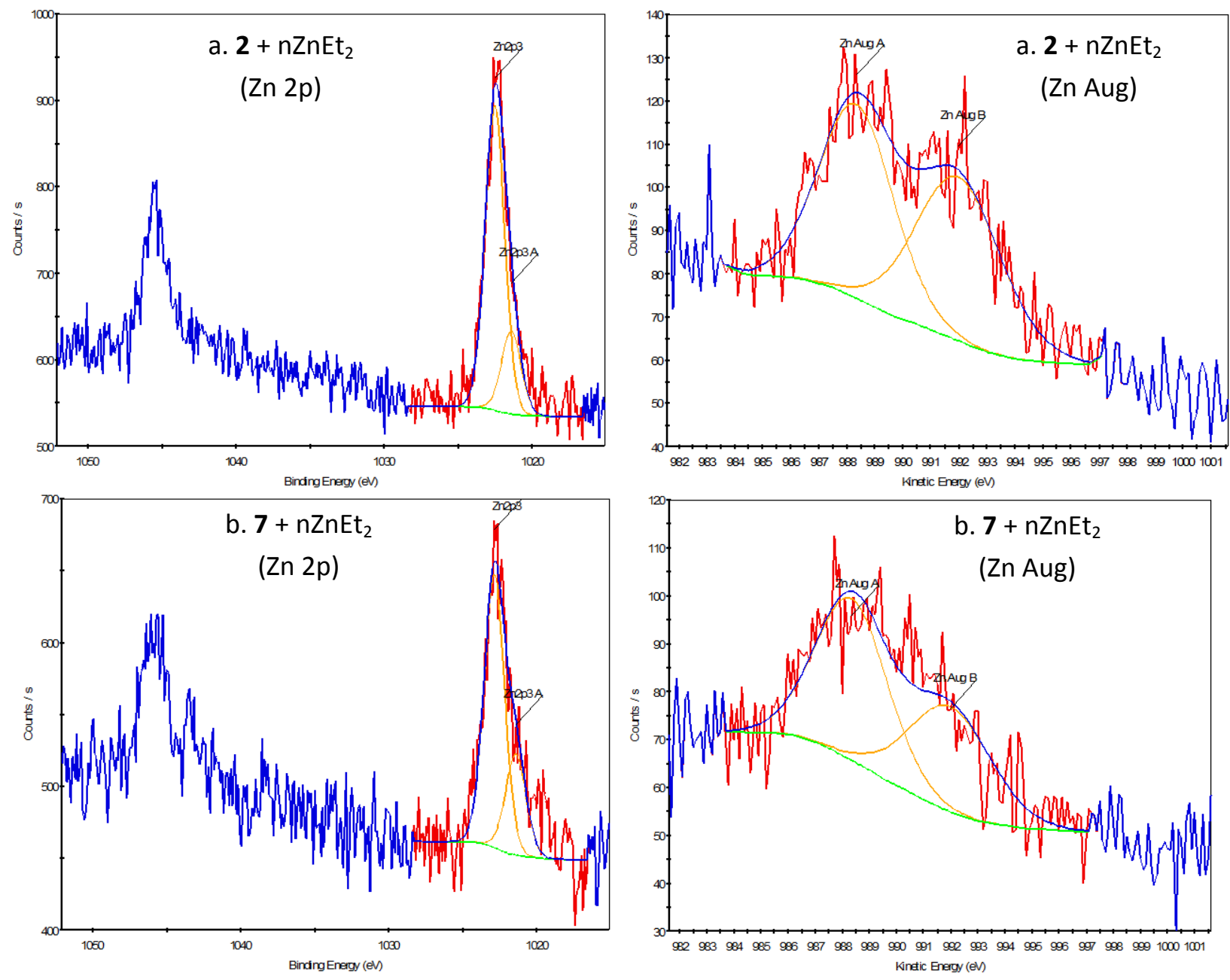

Figure S70: $\quad$ XPS Zn 2p scans (25 scans, $7 \mathrm{~m} 43.8 \mathrm{~s}, 400 \mu$, CAE 50.0) (left) and Zn Auger scans (25 scans, $5 \mathrm{~m} 11.3 \mathrm{~s}, 400 \mu \mathrm{m}, \mathrm{CAE}$ 50.0) (right) for mirrors deposited by the reactions of $\mathrm{ZnEt}_{2}$ with a. [ $\left.\left\{\mathrm{Mn}\left(\mathrm{CH}_{2} \mathrm{CMe}_{3}\right)\left(\mu-\mathrm{CH}_{2} \mathrm{CMe}_{3}\right)_{2}\right\}_{2}\left\{\mathrm{Mn}\left(\mu-\mathrm{CH}_{2} \mathrm{CMe}_{3}\right)_{2} \mathrm{Mn}\right\}\right]$ (2) and b. $\left[\left\{\mathrm{Mn}\left(\mathrm{CH}_{2} \mathrm{SiMe}_{3}\right)\left(\mu-\mathrm{CH}_{2} \mathrm{SiMe}_{3}\right)\right\}_{2}(\mu-\mathrm{dmpm})\right](7)$ 

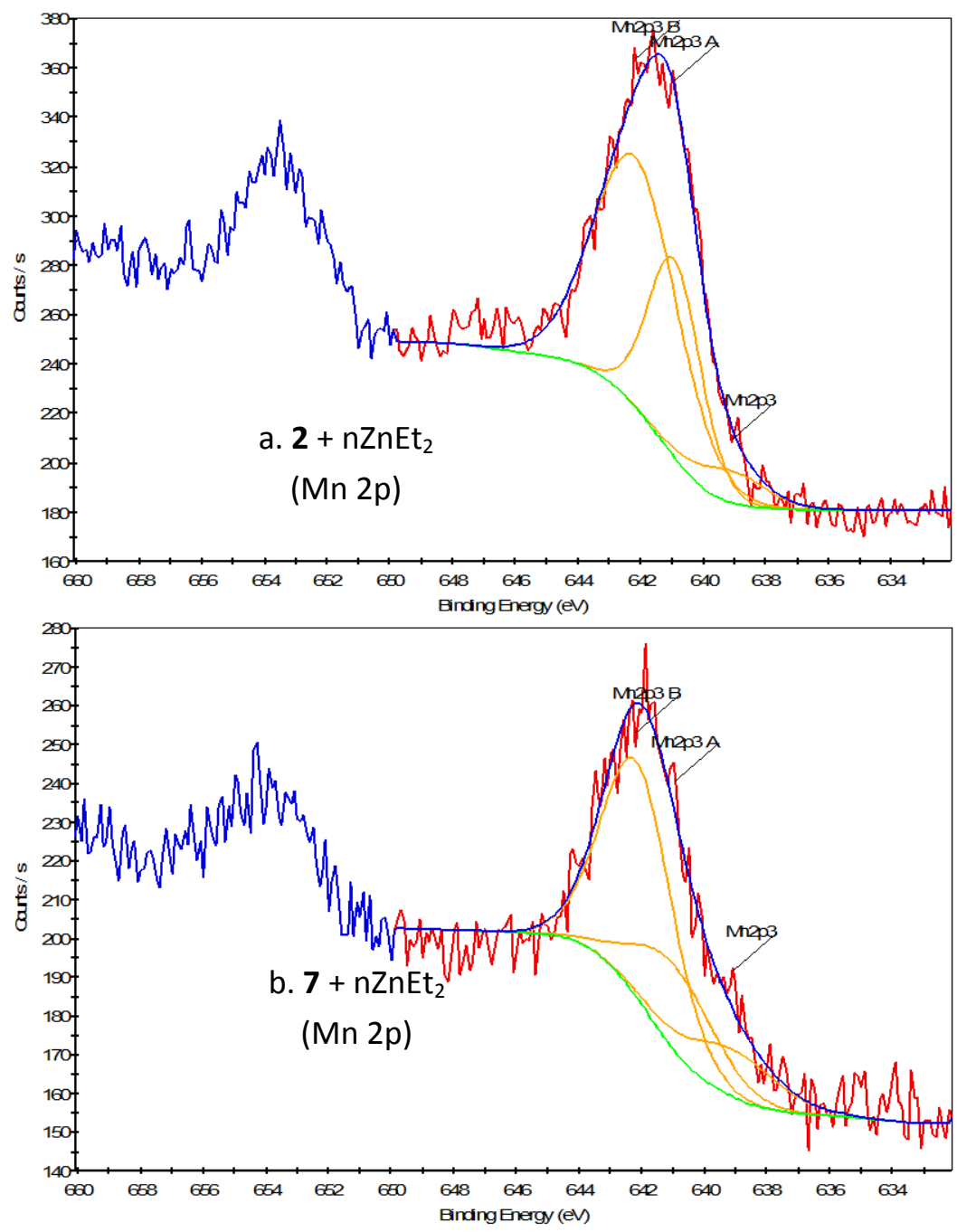

Figure S71: XPS Mn 2p scans for mirrors deposited by the reactions of $\mathrm{ZnEt}_{2}$ with a. $\left[\left\{\mathrm{Mn}\left(\mathrm{CH}_{2} \mathrm{CMe}_{3}\right)\left(\mu-\mathrm{CH}_{2} \mathrm{CMe}_{3}\right)_{2}\right\}_{2}\left\{\mathrm{Mn}\left(\mu-\mathrm{CH}_{2} \mathrm{CMe}_{3}\right)_{2} \mathrm{Mn}\right\}\right] \quad$ (2) and b. $\left[\left\{\mathrm{Mn}\left(\mathrm{CH}_{2} \mathrm{SiMe}_{3}\right)\left(\mu-\mathrm{CH}_{2} \mathrm{SiMe}_{3}\right)\right\}_{2}(\mu-\mathrm{dmpm})\right]$ (7) (100 scans, $23 \mathrm{~m} 25.0 \mathrm{~s}, 400 \mu \mathrm{m}$, CAE 50.0) 


\section{ESI 3 References}

[1] Schubert, E. M., J. Chem. Educ. 1992, 69, 62.

[2] Evans, D. F., J. Chem. Soc. 1959, 2003-2005.

[3] Boeker, G., Phys. Rev. 1933, 43, 756-760.

[4] Bain, G. A.; Berry, J. F., J. Chem. Educ. 2008, 85, 532-536.

[5] Xu, Z.; Thompson, L. K.; Waldmann, O., MAGMUN4.1

[6] Alberola, A.; Blair, V. L.; Carrella, L. M.; Clegg, W.; Kennedy, A. R.; Klett, J.; Mulvey, R. E.;

Newton, S.; Rentschler, E.; and Russo, L., Organometallics 2009, 28, 2112-2118. 